G E U S

Report file no.

22262

GRøNLANDS GEOLOGISKE UNDERSøGELSE

Bulletin No. 110

\title{
GEOLOGIE ET PETROGRAPHIE DES ROCHES
} SEDIMENTAIRES ET VOLCANIQUES KETILIDIENNES (PROTEROZOIQUE INFERIEUR)

DE LA BAIGNOIRE D'ARSUK, GROENLAND MERIDIONAL

PAR

JACQUES MULLER

AVEC 51 FIGURES, 10 TABLEAUX ET 18 PLANCHES

KØBENHAVN

1974 


\title{
GRøNLANDS GEOLOGISKE UNDERSØGELSE The Geological Survey of Greenland

\author{
Øster Voldgade 10, DK-1350 Copenhagen K \\ Denmark
}

\author{
BULLETINS \\ (published in association with the series Meddelelser om Gronland)
}

No. 101 Precambrian organic compounds from the Ketilidian of South-West Greenland. Part IV. 1972 by Jørgen Lam and K. Raunsgaard Pedersen (Meddr Grenland 185, 8).

No. 102 The Røde $\emptyset$ Conglomerate of inner Scoresby Sund and the Carboniferous (?) and Permian rocks west of the Schuchert Flod. A general sedimentological account. 1972 by J. D. Collinson (Meddr Gronland 192, 6).

No. 103 The geology and petrology of the Precambrian rocks to the north-east of the fjord Qagssit, Frederikshåb district, South-West Greenland. 1972 by Giorgio Rivalenti and Antonio Rossi (Meddr Grenland 192,5).

No. 104 A lichenometrical study of snow patch variation in the Frederikshảb district, South-West Greenland, and its implications for studies of climatic and glacial fluctuations. 1973 by G. T. Keith Pitman (Meddr Gronland 192, 7).

No. 105 Stratigraphy of the Jurassic-Lower Cretaceous sediments of Jameson Land and Scoresby Land, East Greenland. 1973 by F. Surlyk, J. H. Callomon, R. G. Bromley \& T. Birkelund (Meddr Gronland 193, 5).

No. 106 The Fiskenæsset complex, West Greenland. Part I. A preliminary study of the stratigraphy, petrology, and whole rock chemistry from Qeqertarssuatsiaq. 1973 by B. F. Windley, R. K. Herd and A. A. Bowden (Meddr Gronland 196, 2).

No. 107 Precambrian ultramafic rocks south of Sermilik, Frederikshåb district, South-West Greenland. 1973 by Zdeněk Misař (Meddr Gronland 196, 3).

No. 108 The Fiskenæsset complex, West Greenland. Part II. General mineral chemistry from Qeqertarssuatsiaq. 1974 by B. F. Windley and J. V. Smith (Meddr Gronland 196,4).

No. 109 Revision of Triassic stratigraphy of the Scoresby Land and Jameson Land region, East Greenland. 1974 by K. Perch-Nielsen, K. Birkenmajer, T. Birkelund and M. Aellen. (Meddr Gronland 193,6).

No. 110 Géologie et pétrographie des roches sédimentaires et volcaniques kétilidiennes (Protérozoïque inférieur) de la baignoire d'Arsuk, Groenland méridional. 1974 par Jacques Muller. (Meddr Gronland 201, 3).

Bulletins of the Geological Survey of Greenland are available on exchange or may be purchased from the Survey. Copies may be purchased as parts of Meddelelser om Gronland from C. A. Reitzels Forlag, Norre Sogade 35, DK-1370 Copenhagen K, Denmark. 
GR ØNLANDS GEOLOGISKE UNDERS ØGELSE Bulletin No. 110

GEOLOGIE ET PETROGRAPHIE DES ROCHES SEDIMENTAIRES ET VOLCANIQUES KETILIDIENNES (PROTEROZOIQUE INFERIEUR) DE LA BAIGNOIRE D'ARSUK, GROENLAND MERIDIONAL

PAR

JACQUES MULLER

AVEC 51 FIGURES, 10 TABLEAUX ET 18 PLANCHES

KØBENHAVN

1974 


\begin{abstract}
The Arsuk $\varnothing$ area is situated along the north-western border of the Early Proterozoic $(>1750$ m.y.) mobile belt of South Greenland. Around Arsuk $\emptyset$ reactivated Archaean (>2500 m.y.) basement is represented by gneiss, amphibolites and migmatites belonging to several lithological series. In the Arsuk basin Early Proterozoic (Ketilidian) supracrustals consist of a group of sedimentary rocks which is overlain by a group of volcanic rocks.

The sedimentary Ikerasârssuk Group, with a thickness between 1000 and $1500 \mathrm{~m}$, consists of semi-pelites and pelites with several zones of pyrite-bearing graphite schists and dolomitic limestones. There are also numerous sills of basic rocks which have the same age as the overlying group of volcanic rocks. In some localities the basal member of the group consists of feldspathic quartzites.

The volcanic Arsuk Group, the upper part of which is eroded away, has a measured thickness of $4200 \mathrm{~m}$. It consists of pillow lavas, basic massive lavas, volcanic breccias, lapillis and tuffites. There are also some ultrabasic rocks and thin horizons of pyrite-bearing graphite schists with chert.

These supracrustal rocks underwent intense deformation at the close of the Early Proterozoic. Three phases can be recognised. The first phase produced N-S to NNE-SSW recumbent folds and the regional schistosity. Refolding during the second phase resulted in folds with $\mathrm{E}-\mathrm{W}$ to ESE-trending axial planes and a strain slip cleavage. The last phase produced $\mathrm{N}-\mathrm{S}$ trending structures.

The grade of metamorphism during the first phase of deformation corresponds to greenschist facies. In the supracrustals close to the basement recrystallisation in amphibolite facies took place between the first and third phases of folding. This shows the existence of a gradient towards still higher grade metamorphic conditions in the underlying Archaean basement undergoing thorough reconstitution at the end of the Early Proterozoic.

As a result of the deformation the stratigraphical unconformity between the Early Proterozoic (Ketilidian) supracrustals and the Archaean basement has been destroyed.

During the Gardar period (Middle Proterozoic: $>950 \mathrm{~m} . \mathrm{y}$. ) and again during the Mesozoic faulting and dyking occurred.
\end{abstract}

\title{
Résumé
}

La région d'Arsuk $\varnothing$ est située au bord NW de la zone orogénique protérozoïque inférieure (>1750 m.a.) du Groenland méridional. Le socle Archéen (>2450 m.a.) est représenté autour d'Arsuk $\varnothing$ par des gneiss, amphibolites et migmatites appartenant à plusieurs séries lithologiques. Dans le bassin d'Arsuk les roches supracrustales d'âge protérozoïque inférieur (kétilidien) comprennent un groupe sédimentaire surmonté d'un groupe volcanique.

Le Groupe sédimentaire d'Ikerasârssuk dont l'épaisseur est estimée à 1000-1500 m, est constitué des roches semi-pélitiques et pélitiques. Des filons-couches basiques, liés à la série volcanique sus-jacente, sont intercalés dans les sédiments. A la base du groupe sédimentaire se trouvent localement des quartzites feldspathiques.

Le Groupe volcanique d'Arsuk a une épaisseur minimale observable de $4200 \mathrm{~m}$. Il est constitué principalement de coulées sous-marines en coussins, de coulées massives de composition basique, de coulées de coussins bréchiques, de lapillis et de tufs. Quelques masses de roches ultrabasiques, ainsi que de minces horizons de roches sédimentaires sont encore intercalés dans les formations volcaniques.

La série sédimentaire et volcanique a été déformée à plusieurs reprises à la fin du Protérozoïque inférieur. On distingue une première phase de déformation (N-S) contemporaine du développement de la schistosité régionale. Cette déformation est accompagnée d'un métamorphisme correspondant au faciès des schistes verts. Les structures de la première phase ont été déformées par des plis ( $\mathrm{E}-\mathrm{W}$ à ESE) de la deuxième phase qui sont accompagnés d'un clivage de crénulation. Ces structures ont été reprises lors d'une troisième phase (N-S). Dans les roches supracrustales situées en bordure du socle on observe de nouvelles cristallisations entre la première et la troisième phase. Ce métamorphisme localisé, correspondant au faciès de l'amphibole, semble lié à une reprise de l'activité plutonique dans le socle.

Les déformations ont eu pour effet de masquer la discordance stratigraphique qui existait entre les gneiss du socle et les roches sédimentaires post-archéennes.

La chronologie des mouvements tardifs, de style cassant (fracturation et mise en place de filons basiques et acides), d'âge Gardar (protérozoïque moyen >950 m.a.) et post-Gardar (mésozoïque) est analysée. 


\section{TABLE DES MATIERES}

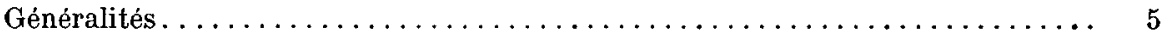

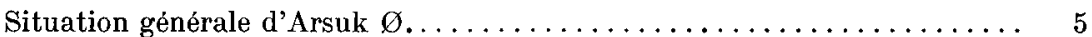

Historique . . . . . . . . . .

Division des roches kétilidiennes dans la région d'Arsuk.......... 12

Définition des termes employés........................ 13

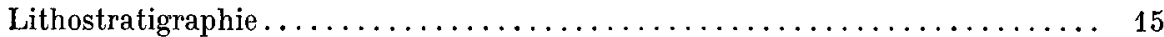

Les divisions lithostratigraphiques. $\ldots \ldots \ldots \ldots \ldots \ldots \ldots \ldots \ldots \ldots \ldots$

Le Groupe sédimentaire d'Ikerasârssuk .................. 16

La Formation de Taylers Havn.................... 16

L'horizon d'Evqitsut....................... 19

L'horizon des quartzites..................... 20

L'horizon des quartzites dolomitiques.............. 27

L'horizon des phyllites rubanés................... 32

L'horizon des schistes sombres.................. 33

La Formation de Taylers Havn sur la côte Nord d'Arsuk $\varnothing \ldots 40$

Conclusion............................. 41

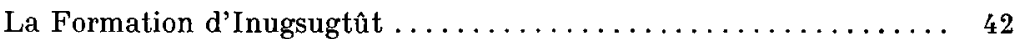

Situation........................ 42

Succession lithologique observée dans la partie supérieure de la Formation d'Inugsugtût à Storø ............... 42

Résumé................................ 43

La Formation d'Isua $\ldots \ldots \ldots \ldots \ldots \ldots \ldots \ldots \ldots \ldots \ldots \ldots \ldots$

La Formation d'Isua sur Arsuk $\varnothing \ldots \ldots \ldots \ldots \ldots \ldots \ldots \ldots, 43$

La Formation d'Isua à Storø ................. 46

Conclusion.......................... 46

Le Groupe volcanique d'Arsuk $\ldots \ldots \ldots \ldots \ldots \ldots \ldots \ldots \ldots \ldots \ldots \ldots \ldots \ldots \ldots$

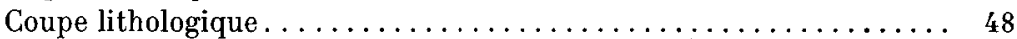

Le matériel volcanique....................... 50

Les coulées de lave avec structures en pillows........... 51

Les coulées de pillows bréchiques ou isolés............. 66

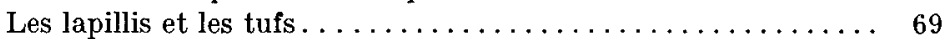

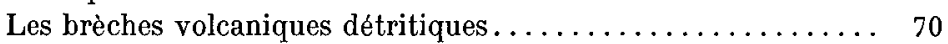

Les laves massives associées aux coulées de pillows........ 70

Les filons-couches basiques dans les formations sédimentaires. . 75

Les filons basiques kétilidiens dans l'édifice volcanique..... 76

Les roches ultrabasiques..................... 77

Le matériel sédimentaire et siliceux dans le Groupe d'Arsuk ...... 79

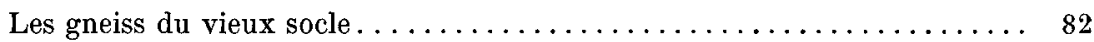

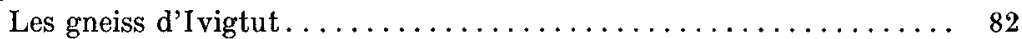

Les gneiss de la côte Nord d'Arsuk $\varnothing \ldots \ldots \ldots \ldots \ldots \ldots \ldots . \ldots 2$

Les gneiss et les amphibolites de l'îlot Narssap sagdlia....... 84 
Les gneiss de la côte Nord-Ouest de Tavdlorutit......... 85

Les gneiss entre Taylers Havn et Taylers Fjeld........... 86

Les gneiss avec enclaves gabbro-anorthositiques............ 87

Les gneiss situés sous les formations d'Arsuk $\varnothing \ldots \ldots \ldots \ldots \ldots \ldots 87$

Les filons basiques discordants dans le vieux socle.......... 88

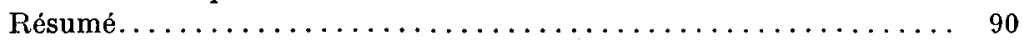

Les minéraux détritiques dans les gneiss et les roches supracrustales. 90

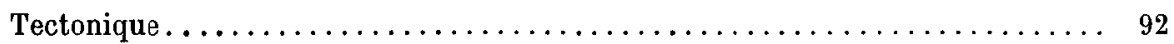

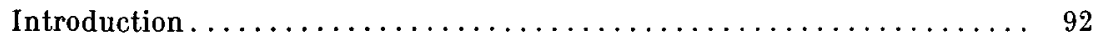

Les éléments tectoniques analysés.................... 93

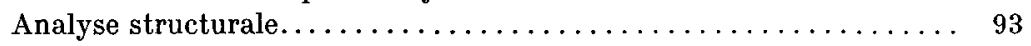

Chronologie des déformations..................... 94

Les déformations kétilidiennes..................... 94

La première phase de déformation: plis contemporains de la schistosité 94

Les plis contemporains de la schistosité dans le groupe volcanique 95

Les structures contemporaines de la schistosité dans le groupe sédimentaire ......................... 98

La deuxième phase de déformation: plis déformant la schistosité.. 101

Les structures dans le complexe tectonique inférieur . . . . 102

La structure synclinale ou en " baignoire " du complexe tectonique

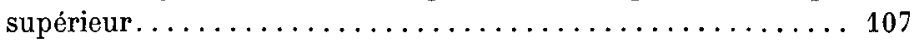

La troisième phase de déformation: plis déformant le clivage . . . . 109

Les structures dans le secteur de Taylers Havn........... 109

Superposition de la $2^{\mathrm{e}}$ et de la $3^{\mathrm{e}}$ phase à Taylers Havn...... 110

Les plis de la côte Nord d'Arsuk $\varnothing \ldots \ldots \ldots \ldots \ldots \ldots \ldots \ldots .111$

La tectonique cassante tardive $\ldots \ldots \ldots \ldots \ldots \ldots \ldots \ldots \ldots \ldots \ldots \ldots \ldots$

Les diaclases plissées....................... 111

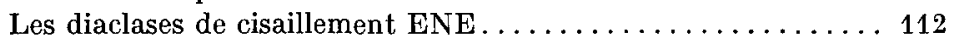

Les contacts entre les roches supracrustales et les gneiss du socle... 112

Les contacts entre Taylers Havn et Taylers Fjeld.......... 112

Les contacts au Nord d'Ikerasârssuk et à Webers Havn...... 113

Résumé............................... 113

Les déformations kétilidiennes dans les gneiss du socle ........ 113

Résumé et conclusion.......................... 114

Les déformations cassantes postkétilidiennes.............. 116

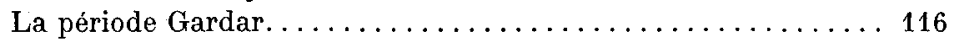

Chronologie des déformations Gardar sur Arsuk $\emptyset \ldots \ldots \ldots \ldots 117$

Les déformations post-Gardar................. 120

Conclusion........................... 121

Le bassin volcano-sédimentaire d'Arsuk et son évolution métamorphique. . . . 123

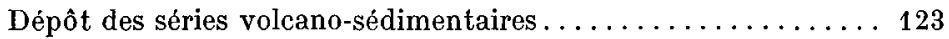

Les cristallisations kétilidiennes. ................ 124

Métamorphisme associé à la $1^{\text {re }}$ phase de déformation....... 129

Les conditions métamorphiques entre la $1^{\text {re }}$ et la $3^{\text {e }}$ phase $\ldots .129$

Le plissement de la $3^{\mathrm{e}}$ phase .................. 130

Recristallisations postérieures à la $3^{\mathrm{e}}$ phase $\ldots \ldots \ldots \ldots \ldots \ldots 130$

Corrélations entre les roches kẻtilidiennes d'Arsuk Ø et de Grænseland-Midternæs 131

Remerciements................................... 133

Liste bibliographique. ........................... 134 


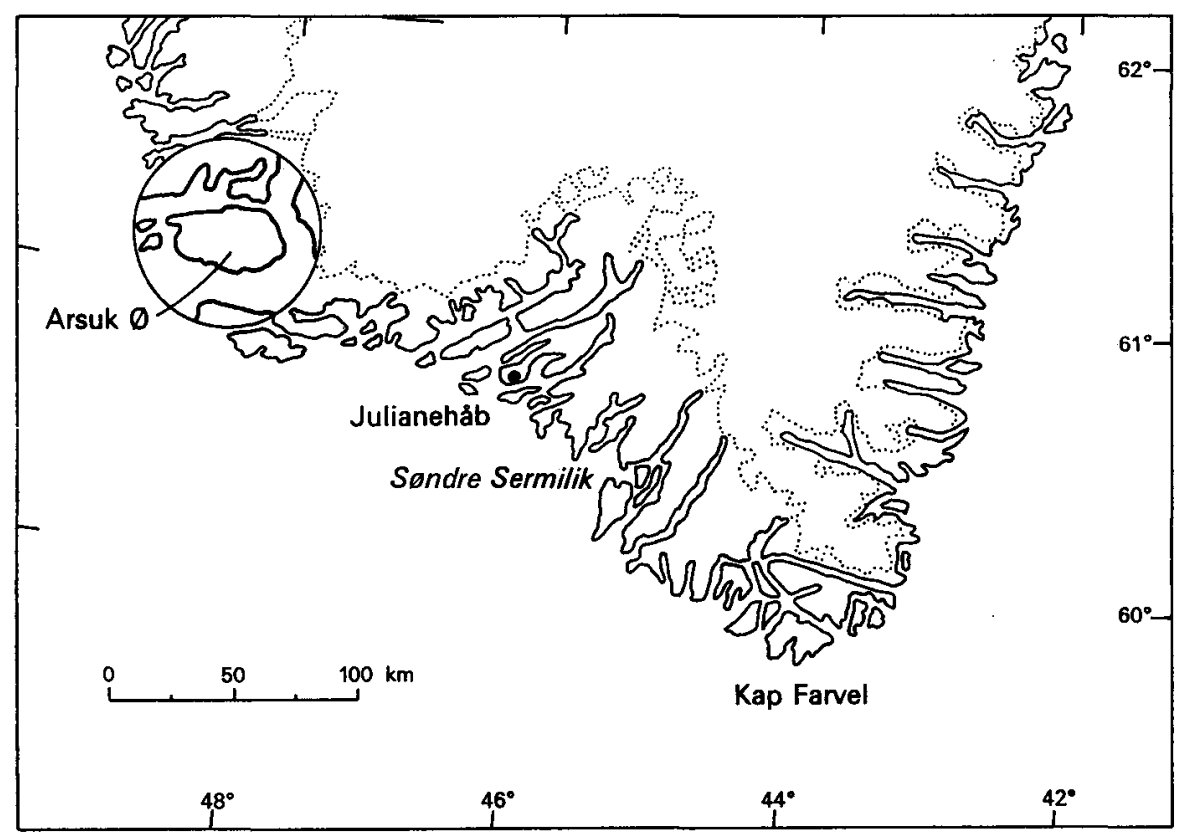

Fig. 1. Carte de la partie méridionale du Groenland avec la situation de la région étudiée.

\section{GENERALITES}

\section{Situation générale d'Arsuk $\emptyset$}

L'île d'Arsuk $\emptyset^{*}$ est située au Nord du $61^{\circ} 05^{\prime}$ de latitude $\mathrm{N}$ et à $l^{\prime} O$ uest du $48^{\circ} 15^{\prime}$ de longitude $\mathrm{W}$ (fig. 1). Elle a une forme trapue, a $12 \mathrm{~km}$ de long sur $6 \mathrm{~km}$ de large, et culmine à $910 \mathrm{~m}$ d'altitude. Cette île se prolonge à l'Ouest par une série de petits îlots.

Les roches composant la région d'Arsuk $\varnothing$ sont d'âge précambrien. On distingue un socle archéen, constitué de gneiss, migmatites, granites et amphibolites, formant la majeure partie des terres situées autour d'Arsuk Ø. Ce socle est surmonté de roches sédimentaires et volcaniques d'âge protérozoïque inférieur appelées kétilidiennes (WeGmans, 1938; HENRIKSEN, 1969).

Les roches sédimentaires et volcaniques, qui constituent le territoire étudié, débordent les limites géographiques d'Arsuk Ø: elles affleurent à Taylers Havn, sur la côte occidentale de la péninsule d'Ivigtut, ainsi que sur les quelques îlots situés au Sud-Est et au Sud d'Arsuk Ø (Kînâlik, Evqitsut). On en trouve également en direction du Sud-Ouest où elles constituent une grande partie de l'île Storø.

$* \varnothing=$ île en danois. 
Deux intrusions granitiques, mises en place à la fin de la période protérozoïque, recoupent les gneiss archéens (sur l'île Quiartorfik) et les roches supracrustales (sur l'île Storø). Une intrusion syénitique, appartenant à la période Gardar (Protérozoïque moyen), constitue le massif du Kûngnât, au Nord du village d'Arsuk.

Les roches archéennes et kétilidiennes sont recoupées par un réseau complexe de filons volcaniques, d'âge Gardar, sauf certains filons appelés TD (Trap Diabase), parallèles à la côte Ouest du Groenland méridional, dont l'âge est probablement mésozoïque (Larsen, 1966).

Les travaux de terrain ont été effectués principalement lors de deux campagnes d'été, en 1957 et 1958, dans le cadre des expéditions annuelles du Service Géologique du Groenland (Grønlands Geologiske Undersøgelse: GGU). En 1961 et 1962, j'ai pu également effectuer de brefs séjours sur Arsuk $\varnothing$.

La mise au point de la plus grande partie du matériel récolté pendant ces expéditions a été faite au Laboratoire du GGU, à Copenhague, entre 1960 et 1963.

\section{Historique}

Ussing définit, en 1912, un " groupe d'Arsuk » qu'il n'a pu reconnaître, au Groenland méridional, que dans la région d'Arsuk. Ce groupe est constitué de roches sédimentaires et volcaniques ("slates, phyllites, dolomites, quartzites, graphitic schists and metamorphosed diabases and tuffs »). Ussin G tente de corréler ces roches avec celles tardi-algonkiennes du Labrador, tandis qu'il attribue à l'archéen les gneiss et les schistes cristallins de la région de Kobberminebugt-Ivigtut (fig. 2).

BøgGild, en 1917, puis Kосн, en 1929, reprennent en héritage la classification de Ussing, mais préfèrent cependant le terme "Arsuk formation " à celui de "Arsuk group ".

Wegmann jette dès 1937 les premières bases de la géologie moderne au Groenland méridional. Elles peuvent être résumées par cette phrase: "la surface topographique du Groenland méridional est entaillée dans les parties profondes d'une très vieille chaîne ". Cette chaîne, qu'il nomme les Kétilides, est constituée d'un vieux socle prékétilidien et d'un matériel plus jeune, kétilidien, de type géosynclinal, qu'il subdivise en deux groupes: un groupe sédimentaire, appelé groupe de Sermilik, surmonté d'un groupe principalement volcanique, appelé groupe d'Arsuk (tableau 1).

Le groupe de Sermilik tire son nom du fjord de Søndre Sermilik (Syd Sermilik de Wegmann) situé à quelque $200 \mathrm{~km}$ au Sud-Est d'Arsuk $\varnothing$, où se trouve une épaisse série de roches sédimentaires surmontées de roches volcaniques. Remarquons que l'âge des roches sédimentaires de Søndre Sermilik est inconnu. Elles peuvent être beaucoup plus vieilles (Dawes, 1970) que celles de la région d'Arsuk. Le Groupe d'Arsuk tire 


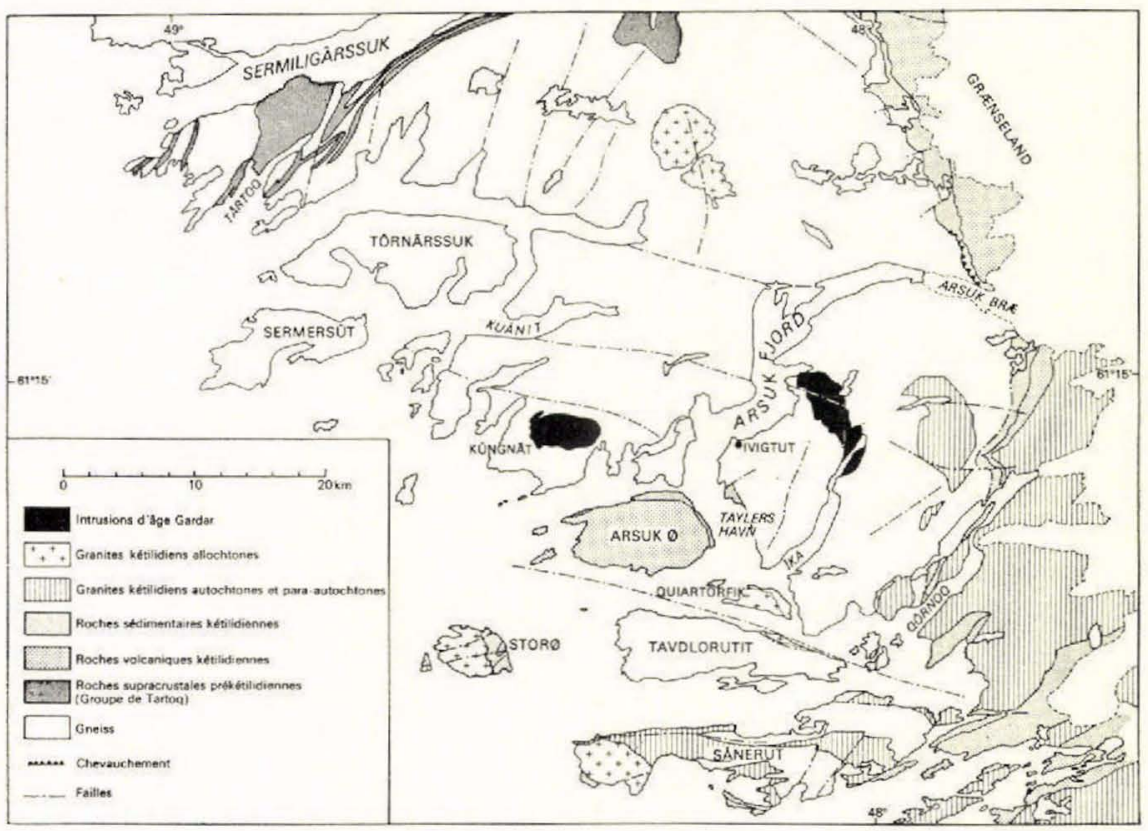

Fig. 2. Carte géologique simplifiée de la région d'Ivigtut.

son nom d'Arsuk $\varnothing$ où il y a également des roches sédimentaires et volcaniques.

L'hypothèse du socle est précisée en 1939. La région d'Arsuk Ø correspond à un synclinal dont le substratum apparaît sur la péninsule d'Ivigtut: "Die Halbinsel von Ivigtut ist zum grössten Teile aus der Unterlage der Mulde aufgebaut. Da das Material stark gemischt ist, kann es nicht als unveränderter alter Sockel angesprochen werden " (WEGMANN, 1939, p. 206). Cette région d'Arsuk appartient, comme celle de Pellinge en Finlande méridionale, à la catégorie "Grundgebirgsmulde» ("synclinal de soubassement »).

En 1948, WEgMANn ajoute: «Les roches du socle prékétilidien apparaissent sous les sédiments de la base des Kétilides et affleurent à plusieurs reprises dans la région du fjord d'Ivigtut, mais aussi plus au Sud. Ce sont des gneiss, des granites et des amphibolites d'un type courant, caractéristiques pour de grandes surfaces des vieilles plates-formes. Ces roches ont subi l'influence du métamorphisme et des granitisations kétilidiennes qui se superposent de cette façon aux vestiges des anciennes transformations » (1948, p. 9).

Callisen, rapportant en 1943 quelques observations faites par GRY en 1931 dans le secteur de Taylers Havn, propose une toute autre hypothèse: les formations supracrustales « rest without discordance on the 
Tableau 1. Chronologie des roches du Groenland méridional (région Ouest) selon Wegman , 1938, 1939 et 1948.

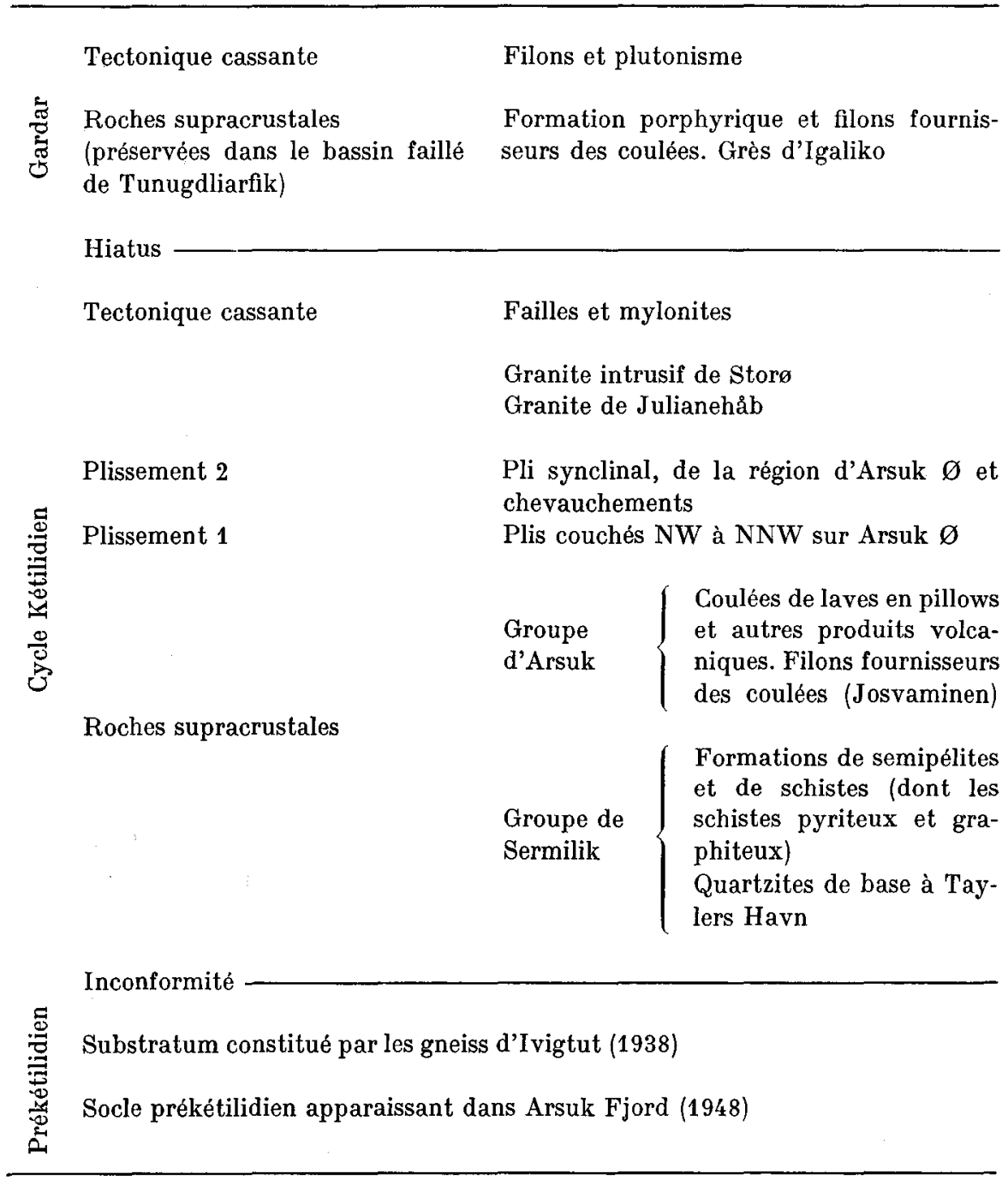

gneiss bed-rock and that the latter formations gradually merge into ... each other.... the difference between the gneiss bed-rock and the crystalline schists on Arsuk Island ... depended on the degree of metamorphism only and that the "gneisses" accordingly were to be considered as migmatites formed of the rocks of the Arsuk group".

On se trouve done en présence de deux hypothèses: les gneiss d'Ivigtut appartiennent en totalité ou en partie à un socle prékétilidien plus ou moins transformé (WEGMANN) ou bien ces gneiss sont le produit 
de la migmatisation de roches sédimentaires et volcaniques kétilidiennes (Callisen-Gry).

Berthelsen, en 1958, 1960 et 1961 présente une première synthèse des travaux cartographiques réalisés par le GGU dans la région d'Ivigtut. Des roches sédimentaires et volcaniques sont découvertes de part et d'autre du glacier d'Arsuk Bræ, depuis Grænseland au Nord jusqu'à Qôrnoq et Sánerut au Sud ainsi que dans la région de Târtoq (fig. 2). A Grænseland elles sont peu métamorphiques tandis qu'à Târtoq, elles sont migmatisées (BERTH ELSEN, 1960, fig. 1). Ces roches, corrélables dans les grandes lignes avec celles d'Arsuk Ø, sont attribuées au Kétilidien.

L'analyse structurale entreprise, tant dans les gneiss et migmatites que dans les roches supracrustales, permet de diviser les roches de la région d'Ivigtut en une infrastructure migmatique et une suprastructure peu métamorphique. La nouvelle chronologie proposée diffère donc de celle de Wegmans par l'absence de socle prékétilidien dans cette région. Les gneiss et les migmatites sont considérés comme des produits de transformation des roches des groupes sédimentaires et volcaniques kétilidiens lors de la migmatisation à la fin du Kétilidien.

Dans la région d'Arsuk, la limite entre l'infra- et la suprastructure est placée sous un horizon de quartzites (quartzites de Taylers Havn) qui se trouve à la base du groupe sédimentaire et qui semble avoir fait obstacle à la migmatisation.

Par contre, dans la région de Târtoq, le front des migmatites atteint des roches volcaniques attribuées au Groupe d'Arsuk. BerThELSEN (1960) pense que les migmatites de cette région proviennent aussi de la transformation des roches des groupes sédimentaires et volcaniques kétilidiens car le rubanement lithologique peut être suivi, à travers le front des migmatites, depuis des roches peu métamorphiques jusque dans les migmatites.

En 1961, Bondesen découvre dans la région de Grænseland une discordance stratigraphique entre les séries kétilidiennes et les gneiss sous-jacents attribués par conséquent au socle archéen (BoNDESEN, 1962; 1970).

Une étude détaillée des séries sédimentaires et volcaniques, qui s'étendent de Grænseland jusqu'à Qôrnoq et Sánerut, montre que ces roches sont beaucoup mieux préservées et développées que dans la région d'Arsuk. Une nouvelle division de ces roches est alors introduite: on distingue, de bas en haut, un Groupe de Vallen principalement sédimentaire, un Groupe de Sortis principalement volcanique et un Groupe de Qipisarqo sédimentaire et volcanique (BONDESEN, 1970).

Selon Berthelsen, (dans Berthelsen \& Noe-Nygaard, 1965), le groupe sédimentaire de la région d'Arsuk serait l'équivalent de la partie supérieure du Groupe de Vallen, tandis que les migmatites et les gneiss 
Tableau 2. Chronologie des roches de la région d'Arsuk $\emptyset$ (avec quelques références aux roches des régions voisines).

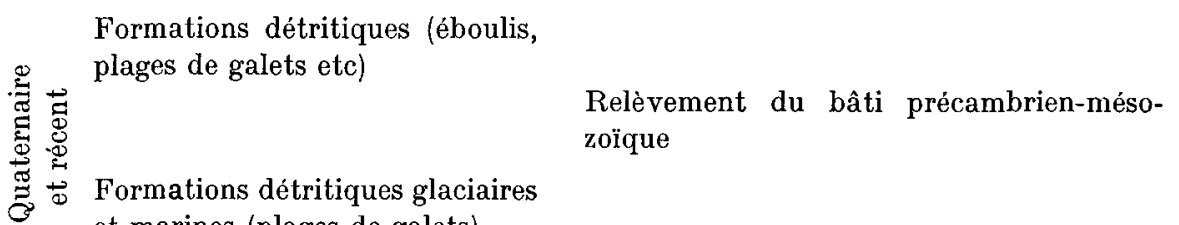
et marines (plages de galets)

inconformité

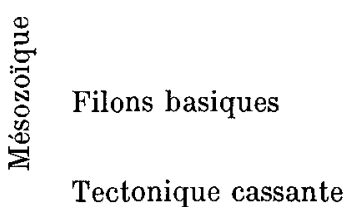

Tectonique cassante
Mouvements de réajustement

Diabases à
olivine $\left\{\begin{array}{l}\mathrm{TD}_{2} \\ \mathrm{TD}_{1} \\ \mathrm{TD}_{0}\end{array}\right.$

Diaclases NNW à N-S hiatus

Mylonites E-W à ENE remobilisation de carbonate, albite etc, minéralisations $\mathrm{Cu}, \mathrm{Pb}$

Décrochements et failles $\mathrm{E}-\mathrm{W}$ à $\mathrm{ENE}$

Tectonique cassante et filons $\approx$ (plutonisme en dehors d'Arsuk Ø)

$\uparrow$ (Accumulation de roches supra( crustales dans le bassin de Tunugdliarfik)

inconformité

Démantèlement de la chaîne kétilidienne

$\downarrow$ Tectonique cassante

(Mise en place de granites intrusifs à Storø, Quiartorfik etc)
Filons de trachyte ENE

Diaclases $\mathrm{NE}$ et filons $\mathrm{BD}_{2}$

Décrochements N-S à NNE

Filons de diabase à olivine $\left\{\begin{array}{l}\mathrm{BD}_{1} \mathrm{E}-\mathrm{W} \\ \mathrm{BD}_{0} \mathrm{E}-\mathrm{W}\end{array}\right.$

Filons à mégacristaux de feldspath et xénolithes

Diaclases E-W et filons lamprophyres

Décrochements et failles E-W à ESE très importants; chevauchements N-S

hiatus

Diaclases de cisaillement ENE

Diaclases plissées

Laminage et discordance structurale entre | socle et Groupe d'Ikerasârssuk.TourmaliniIsation

$\downarrow ?$
Plissement $3^{\mathrm{e}}$ phase (N-S)

Plissement $2^{\mathbf{e}}$ phase $(\mathrm{E}-\mathrm{W}) \quad$ Métamorphisme localisé à la bordure du + clivage

Plissement $1^{\text {re }}$ phase $(\mathrm{N}-\mathrm{S}) \leftarrow$ Métamorphisme régional, faciès des schistes + schistosité socle, faciès de l'amphibole verts 
Tableau 2 (cont.)

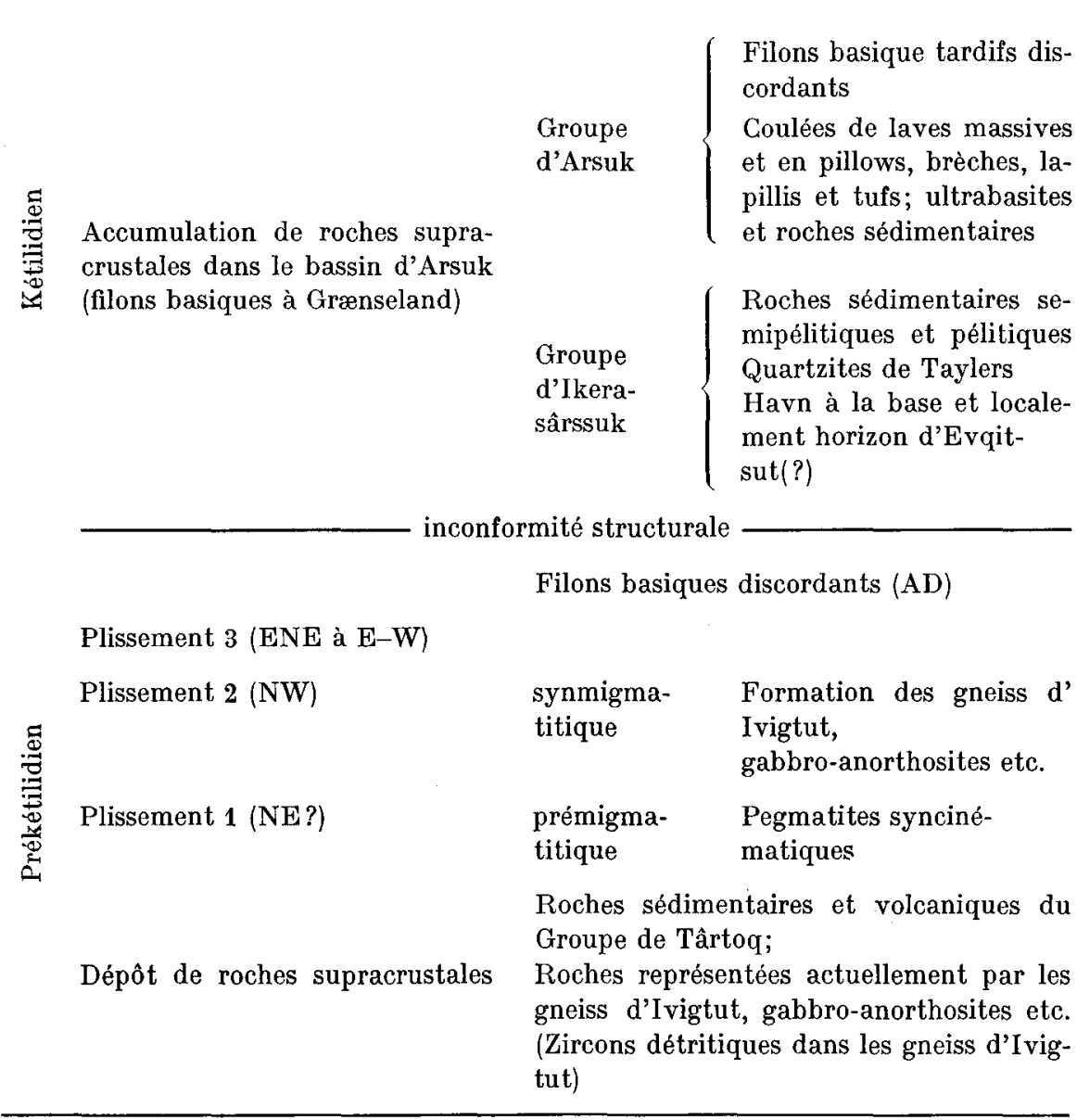

de la péninsule d'Ivigtut proviendraient de la migmatisation de la partie inférieure de ce même groupe. Les gneiss situés sous la discordance à Grænseland appartiendraient, eux, à une écaille du vieux socle en contact tectonique avec l'infrastructure kétilidienne.

Le schéma d'une infra- et suprastructure proposé par BerthelseN va être considérablement modifié à la suite des travaux effectués en 1962 à Midternæs, au Nord de Grænseland. En effet, dans cette région, les roches supracrustales, qui sont le prolongement de celles de Grænseland, reposent en discordance sur un socle constitué de gneiss et de roches supracrustales métamorphisées et migmatisées d'âge archéen. Ces roches supracrustales archéennes migmatisées se poursuivent vers l'Ouest, à Sermiligârssuk et Târtoq. 
Les roches de Târtoq et leur migmatisation sont, par conséquent, archéennes et non pas d'âge kétilidien comme on le croyait jusque là (Higgins \& Bondesen, 1966). La plupart des filons amphibolitiques qui recoupent le socle pourraient être archéens mais quelques uns sont certainement kétilidiens puisqu'on a trouvé, dans la partie centrale de Grænseland, deux filons amphibolitiques traversant la discordance post-archéenne et plissés à la fin du Kétilidien (WindLey et al., 1966).

Par analogie avec les roches de la région de Midternæs-Târtoq, les gneiss et les migmatites de la péninsule d'Ivigtut pourraient appartenir à un socle d'âge archéen (Higgins \& Bondesen, 1966; Henniksens, 1969).

L'étude des formations kétilidiennes d'Arsuk $\varnothing$ et des relations entre ces roches et les gneiss sous-jacents permettra de préciser la chronologie de cette importante région du Groenland méridional (tableau 2).

\section{Division des roches kétilidiennes dans la région d'Arsuk}

Dans l'état actuel des connaissances sur la stratigraphie des séries kétilidiennes du Groenland méridional, il est encore impossible d'assurer que le groupe sédimentaire de la région d'Arsuk correspond exactement à celui qui se trouve en bordure de l'inlandsis, à Grænseland-Midternæs, ou à celui de la région de Søndre Sermilik (Sermilik Group de Wegmann). La même difficulté existe pour le groupe volcanique (Arsuk Group de WEGMANN).

Les régions d'Arsuk et de Grænseland-Midternæs sont séparées par un domaine de gneiss, d'une trentaine de kilomètres de largeur, où aucun témoin de roche post-archéenne n'a été reconnu jusqu'à maintenant. Dans ces deux régions, les séries supracrustales ne sont comparables que dans les grandes lignes.

Les régions d'Arsuk et de Søndre Sermilik sont séparés par un domaine de gneiss et de granite (granite de Julianehåb) s'étendant sur au moins $200 \mathrm{~km}$, où les nombreuses reliques de roches protérozoïques ou archéennes sont peu identifiables (Bridgwater, Escher \& Watterson, 1973).

Afin d'éviter des confusions, il nous semble préférable actuellement de restreindre la notion de groupe lithologique, avec sa qualification locale, à une région bien déterminée où les corrélations sont sûres.

Le «groupe sédimentaire de Sermilik » doit être abandonné dans la région d'Arsuk. Nous le remplacerons par le "Groupe sédimentaire d' Ikerasârssuk 》 du nom du détroit qui sépare Arsuk $\emptyset$ de la presqu'île du Kûngnât à la partie méridionale de laquelle se trouvent préservés quelques lambeaux de roches sédimentaires. 
Nous conserverons le "Groupe volcanique d'Arsuk» puisque WEGMANN (1938) choisit Arsuk $\varnothing$ comme localité type de ce groupe. Cette définition pourrait être restreinte à la seule région d'Arsuk. Suivant le même principe, les Groupes de Vallen, Sortis et Qipisarqo ne devraient être appliqués qu'à l'ensemble de la région de Grænseland-MidternæsQipisarqo-Sánerut où l'on observe pratiquement la continuité des affleurements.

Les fossiles (Vallenia erlingi Raunsgaard Pedersen) découverts dans le Groupe sédimentaire de Vallen, en bordure de l'inlandsis, à Grænseland et Midternæs (Bondesen, 1962; Raunsgando Pedersen, 1966; BONDESEN et al., 1967) permettront peut-être d'établir des corrélations sûres entre les roches kétilidiennes dans la mesure où on les trouvera dans différentes régions du Groenland méridional. Jusqu'à maintenant, les recherches entreprises pour les retrouver dans les séries sédimentaires d'Arsuk $\emptyset$ et de Taylers Havn sont restées vaines (RaunsgaArd PederSEN, 1968).

Les corrélations lithologiques pouvant être établies entre les groupes sédimentaires et volcaniques des régions d'Arsuk et de GrænselandMidternæs sont présentées à la p. 132.

\section{Définition des termes employés}

La stratification est déterminée, dans les formations sédimentaires, par le litage originel des roches. Sur Arsuk $\varnothing$ et à Taylers Havn, les structures sédimentaires de détail ayant disparu du fait des déformations tectoniques et des recristallisations métamorphiques des roches, le litage n'est mis en évidence que par la succession des bancs de nature lithologique variée. Dans le matériel volcanique, le «litage " est donné par l'alternance des coulées de pillow-lavas de forme et de couleur différentes, par l'entassement des pillows au sein d'une même coulée et enfin par les intercalations des tufs et des lapillis souvent granoclassés.

La schistosité correspond à la schistosité de flux de Fourmarier (1949). Le débitage de la roche en feuillets minces s'accompagne d'une recristallisation plus ou moins poussée, parallèlement aux plans des feuillets, des constituants minéralogiques de la roche originellement stratifiée. Cette recristallisation peut être tout à fait discordante à l'ancienne stratification mais dans les flancs des plis serrés, schistosité et stratification sont souvent parallèles.

Le clivage correspond à des zones de fractures de cisaillement discontinues, serrées, presque parallèles entre elles, accompagnées de plissotements, qui traversent la roche déjà schisteuse. Lorsque ces plans de cisaillement sont très marqués, la roche peut se débiter en plaquettes d'épaisseur allant du millimètre à quelques centimètres. Les minéraux 
phylliteux sont déformés et parfois réorientés mécaniquement le long des plans de cisaillement. C'est le "crenulation cleavage" ou le "strain slip cleavage » des auteurs anglais (G. WiLson, 1961).

Au microscope on reconnait parfois des surfaces $S_{0}$ (stratification) qui sont plissées et recoupées par des surfaces $S_{1}$ (schistosité), à leur tour plissées et recoupées par des surfaces $S_{2}$ et parfois même $S_{3}$ (clivages). Les sections minces d'échantillons orientés et replacés dans un cadre structural minutieusement analysé permettent de faire la distinction entre les différentes surfaces $S$ et d'établir une chronologie des déformations et des cristallisations minérales.

Les termes de schistes ou de phyllites ont été utilisés pour définir des roches feuilletées, riches en minéraux phylliteux, assez gros pour être visibles à l'oeil nu (schistes) ou, si petits que leur présence n'est attestée que par l'aspect lustré ou satiné de la surface des feuillets (phyllites) (WinkLer, 1967).

Par convention toutes les mesures de direction sont notées de 0 à $180^{\circ}$ à partir du Nord et dans le sens des aiguilles de la montre (par rapport à l'Est). La notation $075^{\circ}$ signifie Nord $75^{\circ}$ Est par exemple. 


\section{LITHOSTRATIGRAPHIE}

\section{Les divisions lithostratigraphiques}

En l'absence de fossile dans les roches kétilidiennes de la région d'Arsuk $\varnothing$, la stratigraphie présentée ici est basée uniquement sur la succession lithologique des roches. Cette succession a été établie en tenant compte des redoublements des couches dus à la superposition de plusieurs plissements. Dans le groupe sédimentaire, la succession est supposée normale. Ne disposant d'aucun critère sédimentologique, cette hypothèse s'appuie sur des considérations structurales. Dans le groupe volcanique par contre, la forme des pillow-lavas a permis d'établir rigoureusement le sens de la succession (M. E. WiLson, 1942). Les roches kétilidiennes ont été divisées en groupes, formations, unités et horizons qui, suivant Dunbar \& RoDGERS (1957), sont des " unit of rocks in the definition of which the concept of time as such does not enter $»$.

On observe, dans la région étudiée, la succession lithostratigraphique suivante de haut en bas:

Groupe volcanique

d'Arsuk

Groupe sédimentaire
d'Ikerasârssuk $\quad\left\{\begin{array}{l}\text { Formation d'Isua } \\ \text { Formation d'Inugsugtût } \\ \text { Formation de Taylers } \\ \text { Havn }\end{array}\right.$

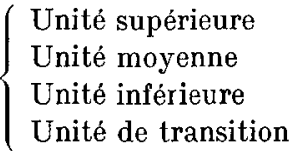

Unité de transition

discordance structurale

Gneiss d'Ivigtut (socle prékétilidien)

Il n'a pas été introduit de nom de formation dans le Groupe d'Arsuk, les quatre unités volcaniques pouvant représenter la totalité des roches constituant ce groupe. Dans toute la région d'Arsuk, il ne subsiste aucun témoin de roche au-dessus de l'unité volcanique supérieure. 


\section{Le Groupe sédimentaire d'Ikerasârssuk}

Le groupe sédimentaire a été divisé dans la région d'Arsuk $\varnothing$ et de Storø en trois formations. Ce sont de bas en haut:

La Formation de Taylers constituée de roches très quartzitiques à la Havn base et devenant de plus en plus phylliteuses vers le haut où se trouvent des roches riches en pyrite et en graphite. On note également la présence de quelques horizons de calcaire dolomitique. Cette formation repose en discordance structurale sur les gneiss d'Ivigtut.

La Formation d'Inugsugtût surtout quartzitique.

La Formation d'Isua constituée de phyllites quartzitiques devenant très pyriteux au sommet.

L'épaisseur du groupe sédimentaire est difficile à évaluer. Nous estimons qu'elle se situe entre 1000 et $1500 \mathrm{~m}$. Cette estimation est délicate, car la mer couvre la plus grande partie des surfaces où devraient affleurer les formations appartenant à ce groupe; en outre, la superposition de plissements a provoqué des étirements et des redoublements de certaines couches dans plusieurs profils.

\section{La Formation de Taylers Havn}

La formation kétilidienne la plus ancienne que nous ayons reconnue dans la région d'Arsuk $\varnothing$ correspond à la Formation psammitique et pélitique de Taylers Havn. Elle est bien visible à l'Est d'Arsuk Ø entre Taylers Havn et Webers Havn. Quelques couches appartenant à la base de cette formation se trouvent encore au Nord du détroit d'Ikerasârssuk (au Nord-Est d'Arsuk Ø), puis à Kvartsitnæs (à l'extrémité Sud-Ouest de la péninsule d'Ivigtut) et enfin sur les îlots Evqitsut et Kînâlik (au Sud et Sud-Est d'Arsuk Ø).

Les roches de cette formation ont depuis longtemps déjà attiré l'attention des géologues. Elles sont signalées par Ussing en 1912. GrY fait, en 1931, quelques observations à Taylers Havn sur le contact avec les gneiss. Wegmann, en 1938, décrit brièvement la succession de Taylers Havn-Webers Havn et son prolongement à Ikerasârssuk (1938, planche 3 et p. 17). Enfin, en 1955, Burri découvre le petit promontoire de Kvartsitnæs.

Du fait de la superposition de plissements accompagnés de schistosité et de clivage, les plans de stratification sont rarement visibles. Dans la 
S

N

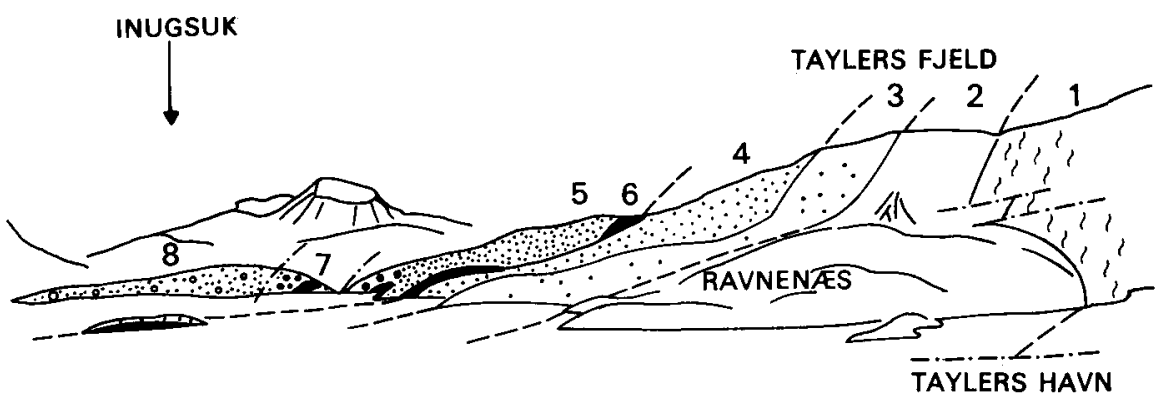

Fig. 3. Succession lithologique observée dans la Formation de Taylers Havn, entre Taylers Havn et Inugsuk. 1: gneiss de la série d'Ivigtut. 2: horizon des quartzites massifs. 3: horizon des quartzites dolomitiques. 4: horizon des phyllites rubanés. 5: horizon des schistes sombres. 6: calcaires dolomitiques. 7: schistes pyriteux et graphiteux. 8: schistes sombres grenatifères. En arrière plan, le massif syénitique du Kungnât.

plupart des cas, le feuilletage observé sur le terrain correspond à la schistosité ou au clivage.

Nous présentons ici la coupe lithologique type, visible entre Taylers Havn et Inugsuk (fig. 3 et 4), en ajoutant quelques observations faites en dehors de ce secteur. La succession est la suivante de bas en haut:

horizon des quartzites

horizon des quartzites dolomitiques

horizon des phyllites rubanés (avec intercalations de calcaire dolomitique)

horizon des schistes sombres (avec les schistes pyriteux et graphiteux)

L'horizon des quartzites constitue la partie inférieure de la Formation de Taylers Havn. Mais sur l'îlot Evqitsut, des couches de calcaire dolomitique, quartzite dolomitique et de phyllite, que nous avons groupées sous le nom d'horizon d'Evqitsut, se trouvent structuralement sous l' horizon des quartzites.

La polarité des couches n'ayant pu être précisée, il n'a pas été possible de déterminer la position stratigraphique de l'horizon d'Evqitsut par rapport à celui des quartzites.

Si la série était inverse, l'horizon d'Evqitsut se situerait stratigraphiquement au-dessus des quartzites. Dans ce cas, il pourrait être l'équivalent de l'horizon des quartzites dolomitiques de la Formation de Taylers Havn.

Dans le cas d'une série normale, l'horizon d'Evqitsut se placerait stratigraphiquement sous celui des quartzites. N'ayant été retrouvé nulle 


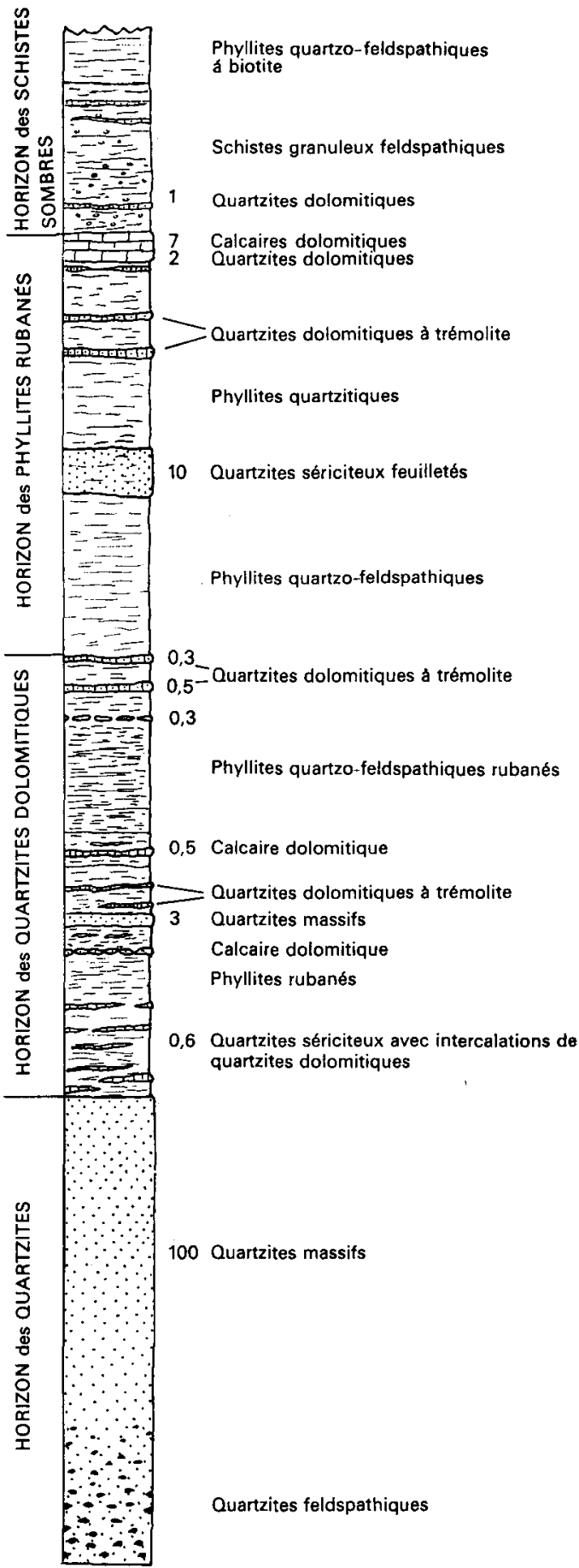

Fig. 4. Succession lithologique observée dans la Formation de Taylers Havn entre Taylers Havn et Fiskebenbugt. Les chiffres indiquent l'épaisseur en métres. 


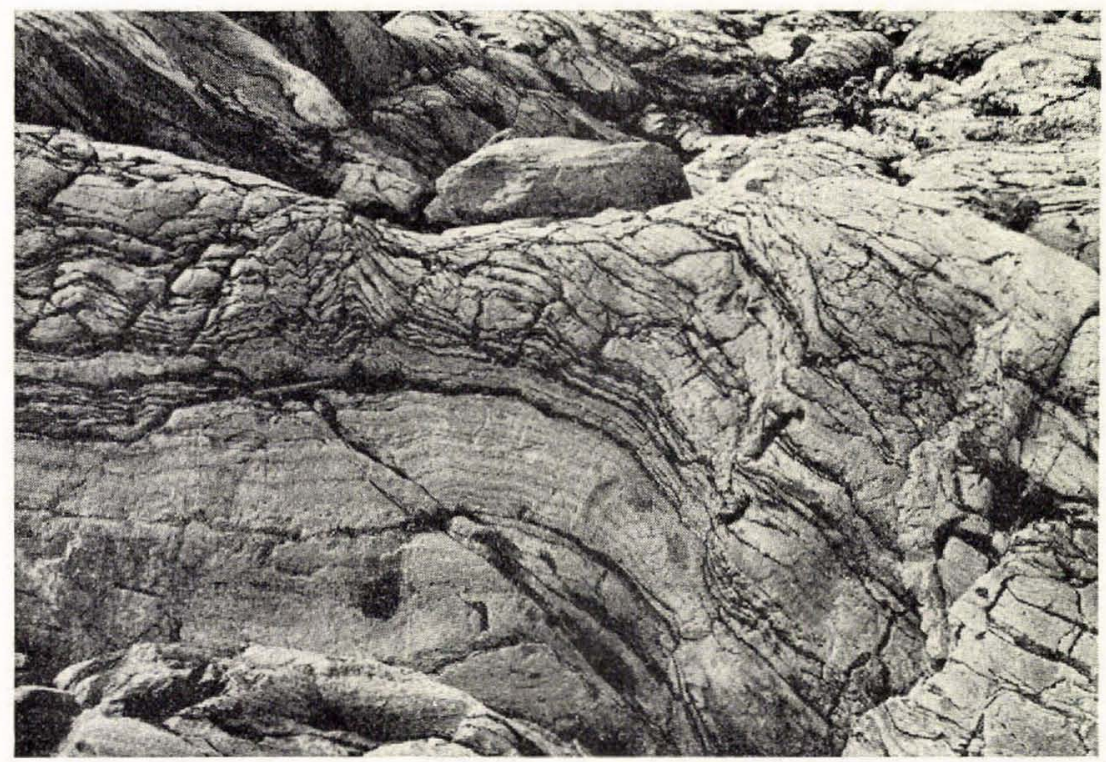

Fig. 5. Quartzites dolomitiques rubanés, sous les quartzites massifs de l'îlot Evqitsut, au Sud d'Arsuk Ø. Les lits plus sombres correspondent à des intercalations carbonatées.

part ailleurs dans le bassin d'Arsuk, il représenterait donc le seul témoin d'un horizon sous-jacent aux quartzites. On pourrait alors se demander ce qu'il est devenu entre Taylers Havn et Webers Havn, là où les quartzites reposent directement sur les gneiss d'Ivigtut. Deux explications sont possibles: soit l'extension géographique de cet horizon est très limitée, soit il a disparu pour des raisons tectoniques, car le contact gneiss-quartzites se fait, partout où il a été possible de l'observer, par l'intermédiaire d'une zone très écrasée.

\section{L'horizon d'Evqitsut}

L'horizon d'Evqitsut est constitué de couches de quartzites dolomitiques de quelques centimètres d'épaisseur alternant avec des calcaires dolomitiques, des niveaux amphiboliques souvent boudinés (trémolite), des calcschistes et des phyllites quartzosériciteux plus ou moins rubanés. Sur l'îlot Evqitsut, les couches sont plissées en un petit synforme dont le coeur est occupé par des quartzites massifs que nous avons rattachés à l'horizon des quartzites de la Formation de Taylers Havn.

L'altération météorologique donne aux couches de quartzites dolomitiques un aspect caverneux par dissolution du carbonate, tandis que les intercalations dolomitiques, qui contiennent toujours un peu de quartz, acquièrent une surface rugueuse (fig. 5). 
L'examen microscopique d'un quartzite dolomitique révèle une alternance millimétrique de lits quartzofeldspathiques, les uns riches en carbonate, les autres sériciteux avec zircons détritiques, sphène leucoxénisé ainsi qu'un peu de minerai de fer. Le feldspath est une albite très fraiche, avec inclusion de quartz, séricite et carbonate. Sa cristallisation, nettement tardive, est probablement liée au métamorphisme régional. Le pourcentage de carbonate (dolomie) varie d'un banc à l'autre entre 5 et 45 .

Les quartzites dolomitiques passent structuralement vers le bas à des phyllites quartzosériciteux gris-vert, plus ou moins rubanés, caractérisés par l'abondance de la séricite qui peut former le $60 \%$ du volume de la roche. La biotite brune ou verte y est peu abondante. Les grains de zircon, arrondis ou ovales, sont souvent très nombreux. On en compte jusqu'à 20 grains par cm² dans certaines sections minces (GGU 54860*).

\section{L'horizon des quartzites}

Les quartzites se trouvent à la base de la Formation de Taylers Havn et reposent sur les gneiss de la série d'Ivigtut, sauf sur l'îlot Evqitsut où, comme nous l'avons vu, ils surmontent peut-être l'horizon d'Evqitsut.

Bien mis en relief par l'érosion glaciaire, les quartzites forment dans le secteur de Taylers Havn un horizon clair, d'une centaine de mètres d'épaisseur. A la cassure fraîche, les quartzites ont une teinte grisâtre, bleuâtre, gris clair ou blanche. L'altération météorologique leur donne une patine ocre ou grise.

La forte recristallisation du quartzite ne permet plus de reconnaitre l'ancienne stratification. Le délitage de la roche en plaquettes est probablement dû à un clivage. Ces plaquettes sont caractérisées par une linéation d'intersection (voir chapitre Tectonique). La surface des plaquettes est couverte de séricite qui peut former localement des lits de 1 à $2 \mathrm{~mm}$ d'épaisseur.

Au point de vue pétrographique, on distingue à Taylers Havn des quartzites feldspathiques (situés à la base de l'horizon) et des orthoquartzites (situés au sommet). A Kvartsitnæs, les quartzites sont traversés par de petites veines de pegmatites.

\section{Les orthoquartzites}

Dans le secteur de Taylers Havn, le feldspath contenu dans les quartzites diminue en quantité et en grosseur lorsqu'on s'écarte du contact avec les gneiss. A une trentaine de mètres du contact, on passe à des orthoquartzites (Pеттілонn, 1957), pauvres en mica et pratiquement dépourvus de feldspath (90 à $98 \%$ de quartz).

* Numéro d'échantillon. 
L'examen microscopique d'un orthoquartzite de Taylers Havn montre que le quartz est complètement recristallisé en plages équidimensionnelles, de 0,2 à $0,4 \mathrm{~mm}$ de diamètre, engrainées, avec sutures marquées. De petites écailles de muscovite sont plus ou moins disposées à la limite de ces plages de quartz; elles s'y trouvent parfois englobées. Les structures détritiques originelles des grains de quartz ne sont pas reconnaissables (planche 1 a).

Sur l'îlot Evqitsut, les orthoquartzites sont mieux conservés. Les grains arrondis de quartz sont bien visibles à la loupe.

$\mathrm{Au}$ microscope, ces grains de quartz se présentent en plages à extinction unique de 0,3 à $0,6 \mathrm{~mm}$ de diamètre, noyés dans une matrice composée essentiellement de quartz en grains dix fois plus petits, avec un peu de carbonate, de muscovite, de zoïsite ainsi que quelques grains de feldspath. Le zircon, arrondi ou ovale, est fréquent. Les grains de quartz, dont la bordure dentelée est due à un accroissement secondaire, sont entourés d'écailles de muscovite. Ces quartz pourraient avoir une origine détrique (planche 1 b).

Les colorations au cobalto-nitrite ont permis de mettre en évidence, dans certains quartzites d'Evqitsut, la présence de deux feldspaths, l'un calcosodique (albite à $5 \%$ An), l'autre potassique. Ces feldspaths se présentent sous deux formes:

En petits grains de $0,05 \mathrm{~mm}$ de diamètre, dispersés dans la matrice, ou bien se développant sous forme poecilitique et englobant alors partiellement des grains de quartz (fig. 6). Cette cristallisation est probablement liée au métamorphisme régional.

En grains beaucoup plus gros, de 0,3 à $0,7 \mathrm{~mm}$ de diamètre, ronds ou ovales, entourés d'écailles de muscovite. Ils sont cassés avec introduction de quartz dans les fissures. Ces feldspaths pourraient être, comme les quartz de même diamètre, d'origine détritique.

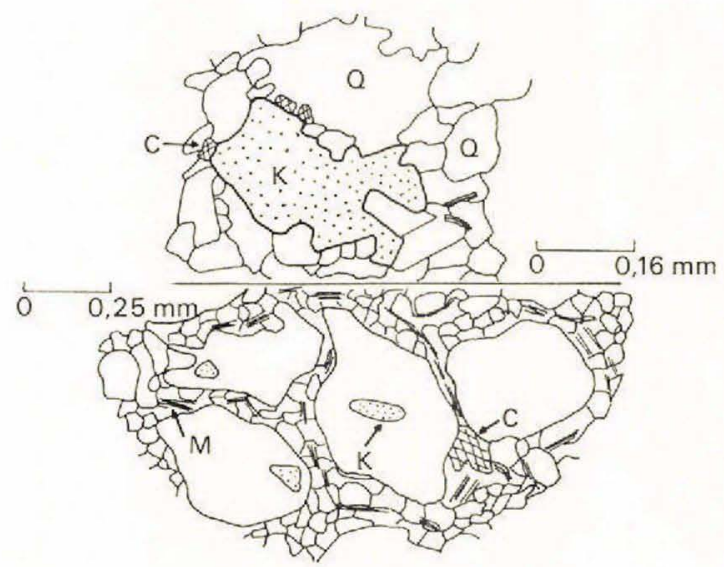

Fig. 6. Micrographie d'un quartzite de la Formation de Taylers Havn. Ilot Evqitsut. Les grains lobés de quartz sont noyés dans une matrice constituée de quartz (Q), carbonate (C) et feldspath potassique (K) qui se développe parfois poecilitiquement. Les écailles de muscovite (M) se disposent autour des grains de quartz (GGU 54859). 
Les quartzites pegmatisés de Kvartsitnæs

Les quartzites gris clair ou blancs de Kvartsitnæs sont traversés de veines pegmatiques de quelques millimètres à plusieurs centimètres $d$ ' épaisseur. Ces veines sont plissées avec les quartzites.

L'examen microscopique d'un quartzite traversé d'une veinule pegmatique plissée montre:

Dans la partie quartzitique, les feldspaths, principalement potassiques, dont le diamètre varie entre 0,1 et $0,5 \mathrm{~mm}$, sont répartis uniformément dans toute la plaque mince. En aucune manière ils ne deviennent plus abondant à proximité de la veine pegmatique. Ils représentent au maximum le 3 à $4 \%$ du volume de la roche.

Dans la veine pegmatique, les feldspaths, principalement potassiques, et la muscovite sont plus abondants et plus gros que dans le quartzite (feldspaths jusqu'à $5 \mathrm{~mm}$ de diamètre).

Dans une veine de texture granitique, d'une dizaine de centimètres d'épaisseur, traversant également les quartzites, les feldspaths sont encore plus abondants que dans les veines pegmatiques. Mais la quantité du plagioclase (albite à oligoclase acide) reste toujours inférieure à celle du feldspath potassique. En outre, ce plagioclase est souvent altéré (formation de séricite, épidote et calcite) alors que le feldspath potassique est toujours très frais.

Toutes les roches de ce secteur sont fortement cataclasées avec fracturation des feldspaths, déplacement des fragments et destruction des structures primaires détritiques des quartz qui sont très recristallisés.

Deux faits peuvent être dégagés de ces observations microscopiques:

Les veines pegmatiques ou granitiques traversant les quartzites sont caractérisées par l'abondance des feldspaths de gros diamètre, de composition potassique principalement et calcosodique.

Le quartzite encaissant est caractérisé par une faible quantité de feldspath généralement potassique, réparti régulièrement dans la roche sous forme de petits grains, sans relation quantitative avec la proximité des veines.

Il n'a pas été possible d'observer si les veines pegmatiques et granitiques étaient limitées aux quartzites (Quirke \& Collins, 1930), ou bien si elles étaient en relation avec les formations gneissiques sous-jacentes. Etant donné la grande quantité de feldspath (calcosodique et potassique) contenu dans ces veines par rapport à la roche encaissante, on peut admettre une migration de matériel feldspathique. Cette migration a fort bien pu se produire à partir du quartzite feldspathique encaissant. Ces veines feldspathiques pourraient correspondre aussi à un remplissage de fissures (feldspaths du type minéraux alpins; WEGManN, communication orale). 


\section{Les quartzites feldspathiques de Taylers Havn}

Entre Taylers Havn et Taylers Fjeld, la base de l'horizon des quartzites est caractérisée, sur une dizaine de mètres d'épaisseur, par la présence de feldspaths, de couleur blanche ou rosée, bien visibles à l'oeil nu. Leur forme ressemble à de petits yeux de la grosseur d'un pépin de pomme ou d'une amande. Les plus gros, parfois subidiomorphes, peuvent atteindre $1 \mathrm{~cm}$ de long sur $0,5 \mathrm{~cm}$ d'épaisseur. Généralement, le grand axe de ces yeux est parallèle au plan de clivage des quartzites. En s'écartant de la base de l'horizon, les feldspaths diminuent en quantité et en grosseur et ne deviennent visibles qu'au microscope. Plus haut dans l'horizon, on passe aux orthoquartzites.

L'étude de sections minces d'échantillons de quartzites feldspathiques, prélevés à des distances variables de la base de l'horizon, permet de dégager les faits suivants:

La recristallisation du quartzite ne permet plus de reconnaître la forme originelle des grains détritiques de quartz. Ce minéral est en plages engrainées de 0,1 à $0,3 \mathrm{~mm}$ de diamètre. Les feuillets de muscovite, fréquemment englobés dans les plages de quartz, sont disposés plus ou moins parallèlement entre eux. Dans de nombreux cas, on observe une division du quartzite en minuscules amygdales de 1 à $3 \mathrm{~mm}$ de long sur $0,5 \mathrm{~mm}$ de large, entourées d'écailles de muscovite. A l'intérieur des amygdales, les grains de quartz ont une extinction roulante marquée et les feldspaths sont cassés. Une partie de la muscovite entourant ces amygdales peut provenir de la séricitisation de feldspaths complètement écrasés. La structure en amygdales, liée à la déformation tectonique de la roche, est probablement due à l'intersection de deux surfaces $S$ qui pourraient être la schistosité et un clivage. Dans les quartzites, ces deux surfaces n'ont pu être différenciées l'une de l'autre au microscope.

La quantité de feldspath (en volume) augmente, dans les grandes lignes, en s'approchant de la base des quartzites. Toutefois, l'examen détaillé montre que cette augmentation est très irrégulière, certaines zones se montrant particulièrement riches en feldspaths, tandis que d'autres semblent en être presque dépourvues; ce phénomène est déjà visible à l'oeil nu.

La grosseur des feldspaths varie beaucoup d'un endroit à l'autre. La plus grande partie du feldspath contenu dans les quartzites est représentée par des cristaux isolés les uns des autres et qui peuvent se grouper en deux types: Les petits feldspaths, peu cataclasés, de forme allongée, dont la longueur varie entre 0,1 et $0,2 \mathrm{~mm}$, leur grand axe étant disposé parallèlement au plan de clivage de la roche. Ils sont presque toujours constitués de feldspath potassique: cristaux 
uniques ou bien maclés, toujours très frais, parfois avec perthites. Leur disposition orientée implique une cristallisation sous effort (planche $1 \mathrm{c}$ ). L'extinction roulante de ces feldspaths montre que cette cristallisation est suivie d'une cataclase.

Les gros feldspaths, ronds ou allongés, parfois subrectangulaires et pouvant atteindre $1 \mathrm{~cm}$ de long (planche $2 \mathrm{a}$ ). Ils sont constitués de feldspath potassique ou calcosodique (albite). Les feldspaths potassiques renferment parfois des inclusions non orientées d'albite. Les perthites en "flammes " sont fréquentes, de même que les antiperthites. Les plagioclases sont souvent séricitisés. Leur pourcentage varie beaucoup d'un endroit à l'autre, mais ils restent toujours, en quantité, inférieurs aux feldspaths potassiques, sauf à la base de l'horizon des quartzites où ils sont alors plus abondants. Les myrmékites sont rares: 2 cas sur environ 500 feldspaths examinés. Les gros feldspaths ont subi les effets d'une cataclase beaucoup plus marquée que les petits individus. Ils acquièrent de ce fait une forme oeillée et sont auréolés de fins débris partiellement transformés en séricite concentrée plus souvent aux deux extrémités des yeux. La cataclase peut aussi les broyer presque complètement.

Ces feldspaths sont probablement des porphyroblastes dont la cristallisation est liée au métamorphisme régional. Ils ont été ensuite cataclasés lors des mouvements tectoniques.

Parmi les constituants certainement détritiques de ces quartzites, on note des zircons, de la tourmaline, du sphène et de la zoîsite. Il est possible que les petites écailles de muscovite entourant les grains détritiques de quartz et de feldspath sur l'îlot Evqitsut aient aussi une origine primaire (BERG, 1952).

Aucune cristallisation authigénique sur noyau arrondi de feldspath n'a pu être reconnue dans les quartzites feldspathiques (GoLdich, 1934; Tester \& Atwater, 1934). Par contre, les cristallisations authigéniques ne sont pas rares dans le cas des tourmalines et plus particulièrement des zircons dont certains appartiennent à deux cycles sédimentaires: croissance, abrasion, croissance authigénique, abrasion. Nous pensons que le premier cycle caractérise le matériel en provenance du socle, alors que le deuxième pourrait correspondre à la formation des quartzites (p. 90).

\section{Comparaison avec quelques quartzites décrits dans la littérature}

Les quartzites de la formation de Taylers Havn présentent beaucoup d'analogie avec des formations de type arkosique décrites dans la littérature. Les quartzites huroniens de Lorrain (Canada) passent d'une composition arkosique à la base de la série, à des orthoquartzites (95 à $99 \%$ de quartz) vers le haut de celle-ci. Ces arkoses reposent même, par endroit, 
directement sur le socle préhuronien (Collins, 1925 et 1914, p. 66). Des arkoses de ce type s'observent également à la base des grès et quartzites d'Igaliko (Groenland) et peuvent se trouver en contact avec le socle kétilidien (Wegmann, 1938; Poulsen, 1964). Les variations locales du contenu en feldspaths sont générales dans toutes les arkoses (РеттілонN, 1957). La nature du feldspath est en étroite relation avec l'origine du matériel et les conditions climatiques. Le plagioclase, dans certains cas, étant plus facilement altérable que le feldspath potassique, le pourcentage de ce dernier est souvent plus élevé. C'est le cas des quartzites de Mississagi et de Serpent (Collins, 1925). Enfin la disposition éparpillée des grains de feldspath est fréquente dans les quartzites de type arkosique. Pour Hietanen (1938), cette disposition serait une indication de l'origine sédimentaire d'une partie du feldspath contenu dans les quartzites granitisés d'Olostunturi, en Laponie occidentale.

Quirke \& Collins (1930) ont décrit différents stades de transformation des quartzites huroniens de Lorrain, granitisés par le granite de Killarney. Le quartzite originel, feldspathique, est altéré (feldspath séricitisé), schistosé et recristallisé. Un premier stade de transformation est le "quartzite porphyry", caractérisé par l'apparition de phénocristaux de quartz et de feldspath (ayant jusqu'à $2 \mathrm{~mm}$ de diamètre). Ce feldspath, ainsi que celui qui constitue le tiers de la matrice, est surtout potassique; il provient en partie de la reconversion du mica blane et en partie d'apport extérieur. Le "porphyry » est caractérisé par l'apparition de la biotite et de la magnétite et par l'augmentation du pourcentage de feldspath; on passe ensuite au "porphyry gneiss" où les phénocristaux de feldspath occupent $60 \%$ du volume de la roche qui acquiert une foliation marquée. Le stade extrême est représenté par le granite de Killarney, où $90 \%$ du volume de la roche est constitué principalement de larges cristaux de feldspath (jusqu'à $5 \mathrm{~mm}$ de diamètre), d'un peu de quartz et de biotite.

Si le « quartzite porphyry " ressemble à certains quartzites de Taylers Havn et d'Evqitsut (présence de phénocristaux de quartz et de feldspath), il en diffère néanmoins, comme les autres produits de transformation, par la plus grande quantité de feldspath constituant sa matrice (dans les quartzites de Taylers Havn, le feldspath est principalement sous forme de cristaux isolés). Il en est de même des " quartzitic gneiss » et « fine grained gneisses ", décrits par Eskola \& Nieminen (1938) et qui sont aussi, selon ces auteurs, des produits de granitisation de roches quartzitiques plus ou moins arkosiques.

\section{Résumé et conclusion}

Dans les quartzites feldspathiques de la Formation de Taylers Havn, la cataclase suivie d'une forte recristallisation du quartz a masqué com- 

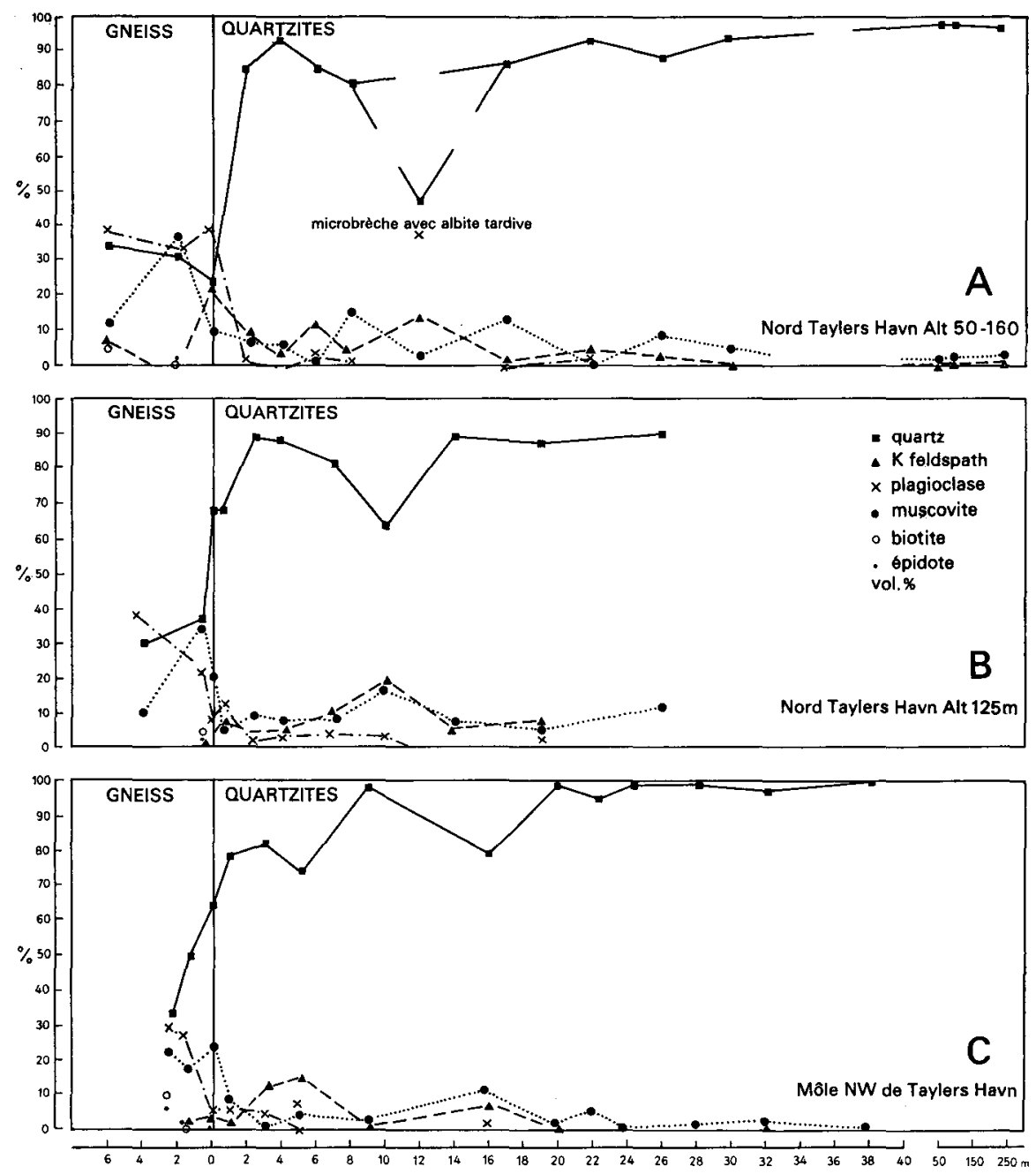

Fig. 7. Contact gneiss d'Ivigtut-quartzites de Taylers Havn. Composition minéralogique.

plètement les relations originelles existant entre les deux constituants principaux qui sont le quartz et le feldspath.

Plusieurs hypothèses permettent d'expliquer la présence de feldspath dans ces quartzites: soit par la nature arkosique du sédiment originel, soit par néoformation liée au métamorphisme ou à la granitisation. Ces deux possibilités peuvent aussi se superposer.

Les feldspaths dispersés sans orientation préférentielle dans une matrice de quartz pourraient avoir une origine détritique. Comme nous l'avons $\mathrm{vu}$, les feldspaths ne deviennent ni plus nombreux ni plus gros au voisinage immédiat des veines pegmatiques ou granitiques traversant les quartzites. Ils sont également présents avec les mêmes caractères dans des quartzites non traversés de veines pegmatiques. 
Une partie des feldspaths a dû aussi se former aux dépends de la muscovite, si l'on considère la fig. 7 , diagramme $A$, où il y a diminution du pourcentage de la muscovite quand le feldspath potassique augmente. Ce phénomène est cependant un peu moins évident sur les autres diagrammes. Par cristallisation à partir du matériel primitif, on observe dans certains cas la néoformation de petits feldspaths, principalement potassiques, allongés parallèlement aux feuillets de muscovite. Il n'a pas été possible de définir la période de cristallisation de ces feldspaths par rapport à la schistosité ou au clivage affectant les quartzites car ces deux surfaces sont souvent subparallèles. Il est probable que cette cristallisation ait contribué à modifier la structure des gros feldspaths comme Hietanen (1938) l'observe, par example, dans les quartzites arkosiques de Olostunturi, en Finlande: «During the metamorphism a complete recristallisation has taken place and, in this recristallisation, the feldspar has produced more idiomorphic shapes than the quartz ". Les recristallisations partielles observées dans les gros feldspaths, telles que les cicatrisations de fissures, ont pu s'effectuer à ce stade ainsi que la formation des veines pegmatiques et granitiques dans les quartzites de Kvartsitnæs.

Le passage des "arkoses 》 aux orthoquartzites, caractérisé par un "haut degré de maturité texturale et minéralogique 》 (Ретітыонn, 1957), pourrait s'expliquer ici par lavage et vannage répétés du sable dans la zone du rivage avec destruction et élimination consécutive du matériel feldspathique.

\section{L'horizon des quartzites dolomitiques}

Nous rassemblons sous cette dénomination un ensemble hétérogène de roches, parmi lesquelles les quartzites dolomitiques dominent, mais où se trouvent également des phyllites quartzofeldspathiques rubanés.

Dans la région de Taylers Havn, l'horizon des quartzites dolomitiques a une épaisseur de 80 à $100 \mathrm{~m}$ environ. La base de cet horizon est constituée principalement par des quartzites riches en carbonate et amphibole, avec quelques petits niveaux de phyllites quartzofeldspathiques. Ces derniers deviennent plus abondants dans la moitié supérieure de l'horizon qui se termine par quelques petits bancs de quartzites carbonatés. Les phyllites, qui prennent dans l'horizon suivant un développement plus considérable, correspondent aux «schistes gneissiques» de WEGmanN (1938).

L'horizon des quartzites dolomitiques se retrouve seulement au Nord du détroit d'Ikerasârssuk; partout ailleurs, il est masqué par la mer.

\section{Les quartzites dolomitiques}

A Taylers Havn, les quartzites dolomitiques sont constitués de niveaux quartzitiques plus ou moins carbonatés, de 2 à $15 \mathrm{~cm}$ d'épaisseur, alternant avec des niveaux d'épaisseur semblable de calcaire dolomitique 


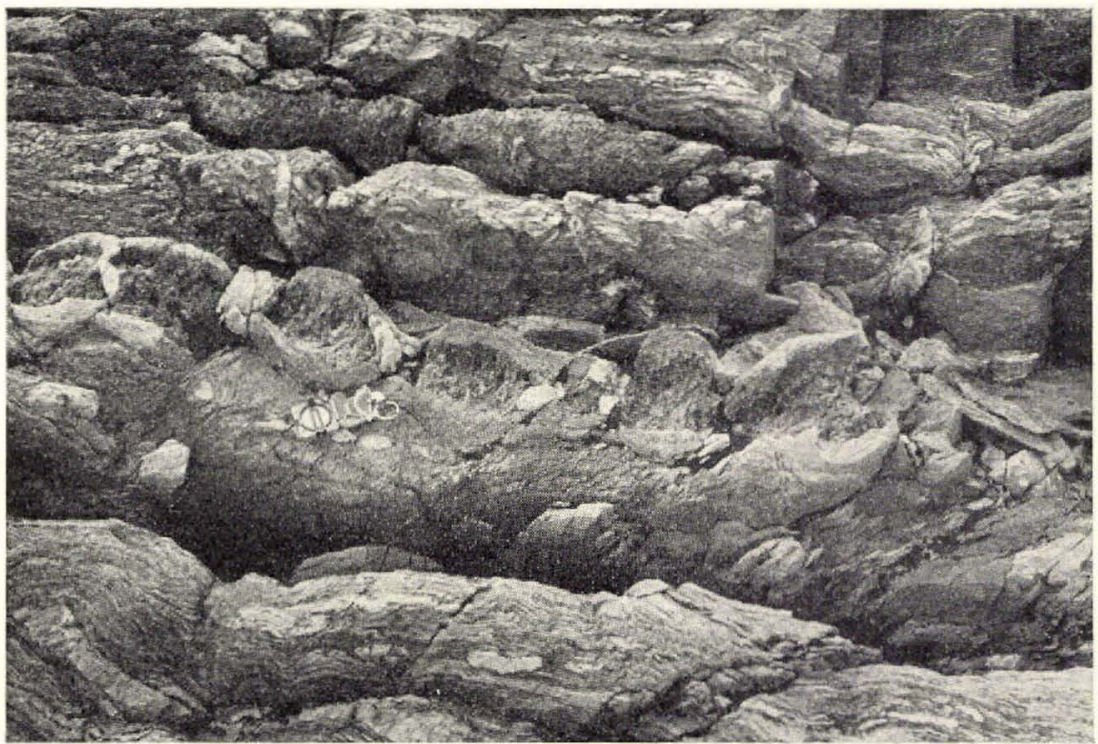

Fig. 8. Quartzites carbonatés amphiboliques rubanés alternant avec des phyllites quartzitiques. Horizon des quartzites dolomitiques à Taylers Havn. Les lits amphiboliques sont boudinés. Remplissage de quartz entre les boudins.

beige clair, parfois un peu gréseux ou encore de niveaux amphiboliques (trémolite) gris-vert clair; ces derniers sont souvent boudinés (fig. 8). La quantité de carbonate contenue dans les lits quartzitiques varie beaucoup d'un endroit à l'autre, mais ne dépasse guère $20 \%$ en volume.

L'examen microscopique d'un quartzite dolomitique révèle les caractères suivants:

Le carbonate se trouve entre les plages de quartz très recristallisé de $0,5 \mathrm{~mm}$ de diamètre. Aucune structure détritique primaire n'est visible.

La muscovite est en toutes petites écailles, plus ou moins allongées suivant la schistosité.

L'amphibole, incolore, fibreuse (trémolite), est disposée en gerbes s'allongeant de préférence suivant la schistosité. Certaines gerbes se développent cependant tout à fait obliquement.

Le feldspath (albite principalement) est fortement granulé et séricitisé. Mèlé à du quartz également granulé, il forme des lits irréguliers contenant quelques zircons détritiques. Ces lits alternent avec des lits constitués essentiellement de quartz recristallisé en plages équidimensionnelles (ségrégation).

Dans les niveaux plus amphiboliques, la trémolite se développe en larges cristaux poecilitiques englobant des grains désorientés de carbonate. Dans la partie externe des boudins amphiboliques, la trémolite fortement cataclasée est transformée en talc. Parfois elle est biotitisée (biotite peu colorée). A l'intérieur des boudins, la cataclase est faible et l'amphibole reste stable. 
$S$

ARSUK $\emptyset$

$\mathrm{N}$

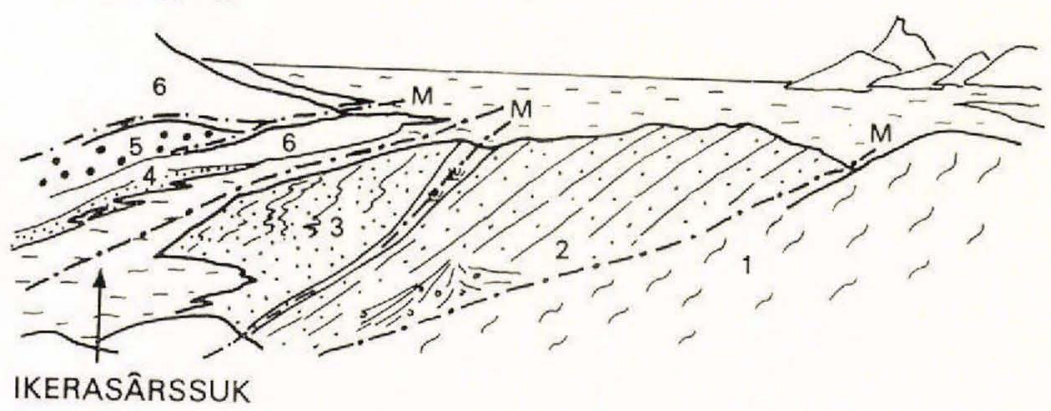

Fig. 9. Succession lithologique observée dans la Formation de Taylers Havn dans le secteur d'Ikerasârssuk. 1: gneiss de la série d'Ivigtut. 2: quartzites massifs. 3: quartzites dolomitiques. 4 : schistes sombres, calcaires dolomitiques et phyllites pyriteux. 5: schistes pyriteux et graphiteux. 6: filons-couches de gabbro-diorite. Le contact entre les gneiss et les quartzites se fait par l'intermédiaire d'une zone mylonitique.

Les niveaux carbonatés qui se trouvent de part et d'autre des lits amphiboliques boudinés sont constitués de calcite et de dolomie et contiennent aussi un peu de quartz $(2-3 \%)$. Le mica pléochroïque, peu coloré ( $\alpha=$ incolore; $\gamma=$ brun-jaune clair) et la chlorite sont présents en faible quantité.

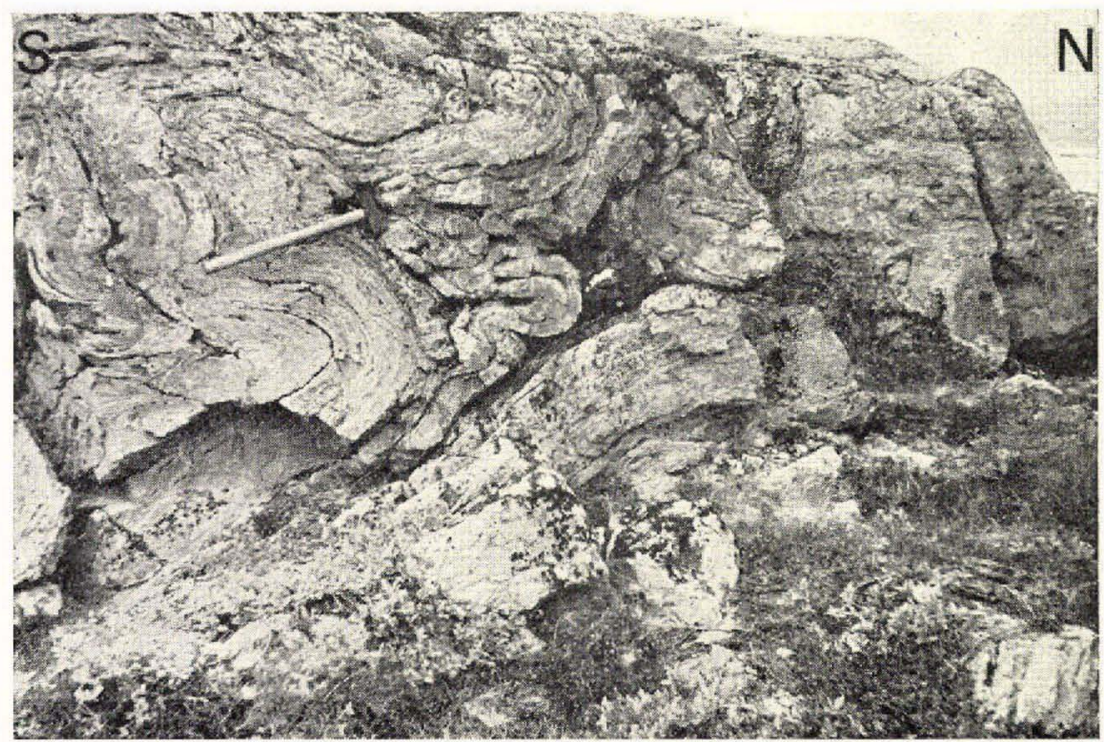

Fig. 10. Horizon des quartzites dolomitiques de la Formation de Taylers Havn, au Nord du détroit d'Ikerasârssuk. Quartzites dolomitiques avec intercalations carbonatées plissées disharmoniquement. Les axes de plis horizontaux ont une direction $070^{\circ}$. Les plis sont déversés au Sud. 
A Ikerasârssuk, les quartzites massifs gris ou noirs contiennent un peu de carbonate. La base de l'horizon n'est pas connue, car elle est recoupée par la mylonite d'Ikerasârssuk-Webers Havn (fig. 9). Ces quartzites massifs sont surmontés de quartzites dolomitiques extrêmement plissés (fig. 10), caractérisés par la présence de très minces lits discontinus de dolomie avec un peu d'amphibole. On y observe également des intercalations de plusieurs décimètres d'épaisseur de calcaire dolomitique grisâtre contenant par endroit jusqu'à $50 \%$ de quartz. Comparé aux quartzites dolomitiques du secteur de Taylers Havn, le développement d'amphibole est assez faible dans toutes ces roches. La composition originelle des sédiments en est probablement la cause.

\section{Les phyllites quartzofeldspathiques rubanés}

Les phyllites quartzofeldspathiques, très plissés, sont constitués d'une alternance de lits micacés, d'épaisseur millimétrique, sombres, irréguliers et discontinus et de lits quartzitiques ou quartzofeldspathiques clairs ayant jusqu'à $2 \mathrm{~cm}$ d'épaisseur.

Cette alternance correspond à la schistosité. Les fortes recristallisations qui se sont encore produites le long de plans de schistosité masquent la stratification. Par endroit, on observe que cette schistosité est déformée par des petits plis extrêmement serrés accompagnés de clivage de plan axial, ce qui explique la discontinuité des lits de composition différente. Les flanes des petits plis sont à leur tour déformés par des plis d'entraînement de style en " accordéon " liés à la structure synclinale N-S de Taylers Havn. Ces déformations ont eu pour effet un redoublement, au moins local, des couches de phyllites quartzofeldspathiques.

Tableau 3. Composition minéralogique des phyllites quartzofeldspathiques.

\begin{tabular}{|c|c|c|c|c|}
\hline \multirow[b]{2}{*}{$n^{\circ}$ plaques GGU } & \multicolumn{2}{|c|}{$\begin{array}{c}\text { Horizon des quartzites } \\
\text { dolomitiques }\end{array}$} & \multicolumn{2}{|c|}{$\begin{array}{c}\text { Horizon des phyllites } \\
\text { rubanés }\end{array}$} \\
\hline & 54875 & 54870 & 54873 & 54887 \\
\hline & $\%$ & $\%$ & $\%$ & $\%$ \\
\hline Quartz........ & 36 & 25 & 25 & 46 \\
\hline Feldspath............. & 20 & 33 & 36 & 27 \\
\hline Muscovite............ & 27 & & 11 & n \\
\hline 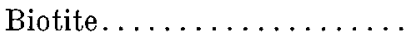 & 11 & 33 & 10 & 19 \\
\hline Epidote............. & 4 & 7 & 16 & \\
\hline Minéraux secondaires . . . . . . & 2 & 2 & 2 & 8 \\
\hline
\end{tabular}

La composition minéralogique des phyllites est donnée dans le tableau 3.

Sous le microscope, ces roches sont caractérisées par: 
L'abondance de micas répartis dans des lits discontinus et "en relais". Les feuillets micacés sont disposés parallèlement à la schistosité. Exceptionnellement, il est possible de reconnaître d'anciennes charnières de plis dont les flancs sont parfois limités par des plans de clivage qui permettent d'expliquer la disposition lenticulaire et "en relais " des lits micacés. La muscovite est prédominante sur la biotite verte.

Les feldspaths calcosodiques et potassiques, ainsi que le quartz, sont présents dans toute la roche; leur granulation est due à une forte cataclase. La muscovite provient certainement pour une part de la séricitisation du matériel feldspathique. On observe également une cristallisation de feldspath perthitique, très frais, qui s'allonge entre les feuillets micacés et contient des inclusions de muscovite. Ces longs feldspaths ont subi ensuite une cataclase due au plissement en " accordéon " (fig. 47). L'ilménite, plus ou moins leucoxénisée, s'allonge aussi suivant la schistosité.

L'épidote (une pistacite zonée à noyau d'orthite) est en larges cristaux subidiomorphes cataclasés recoupant parfois les plis en "accordéon".

Le quartz, recristallisé, est concentré en lits lenticulaires subconcordants avec la schistosité. Ces lits épousent la forme des plis en "accordéon" tout en les recoupant par endroit (planche 2b). Ils renferment de nombreuses inclusions de tous les minéraux précédents. Une telle disposition correspond au "quartz imbriqué, discordant et lamellaire ", défini par Belliere (1958, p. 83). On assiste ainsi à une remobilisation du quartz qui se concentre d'abord dans toutes les zones de discontinuité de la roche, telles que les plans de schistosité et de clivage. Cette remobilisation dépasse le cadre de l'échantillon et affecte des bancs entiers.

Le zircon et la zoïsite se présentent en grains ronds ou ovales.

\section{Résumé et conclusion}

Le rubanement des phyllites quartzofeldspathiques ne correspond pas à la stratification mais à la schistosité. Celle-ci est déformée par deux phases successives de plissement, l'une correspondant à l'apparition d'un clivage recoupant la schistosité et l'autre étant caractérisée par la déformation de ce clivage par des petits plis d'entraînement liés à la structure N-S du synclinal de Taylers Havn.

L'amphibolisation des calcaires et des quartzites dolomitiques est probablement tardive par rapport à la formation de la schistosité. La cristallisation des feldspaths perthitiques dans les phyllites quartzofeldspathiques est antérieure à la formation des plis d'entraînement en "accordéon ". Elle pourrait être synchrone de la formation du clivage, car ces longs feldspaths ne semblent avoir subi que la cataclase liée à la formation des plis en "accordéon".

Le boudinage des lits amphiboliques, la transformation des amphiboles en talc et la cristallisation de l'épidote dans les phyllites se sont produits lors du plissement qui a donné naissance à la structure synclinale $\mathrm{N}-\mathrm{S}$ de Taylers Havn et qui correspond à la dernière phase. La ségrégation de quartz « discordant et lamellaire " est postérieure à cette phase de plissement.

La succession lithologique et la composition pétrographique des roches de l'horizon des quartzites dolomitiques présentent beaucoup d'analogie avec celles de l'horizon d'Evqitsut. S'il était possible de prouver 
que la série visible sur l'îlot Evqitsut est en position inverse, il faudrait évidemment abandonner le terme horizon d'Evqitsut.

\section{L'horizon des phyllites rubanés}

Cet horizon est composé essentiellement de phyllites quartzofeldspathiques plus ou moins rubanés. Au sommet de l'horizon, on retrouve quelques bancs de quartzites carbonatés et amphiboliques qui passent ensuite à des calcaires dolomitiques.

\section{Les phyllites rubanés}

La nature de ces roches est très semblable à celle des phyllites décrits dans l'horizon sous-jacent. Ici les lits leucocrates sont un peu plus épais et atteignent fréquemment $5 \mathrm{~cm}$ d'épaisseur. Les résultats de l'analyse planimétrique au compteur de points de quelques phyllites quartzofeldspathiques sont donnés dans le tableau 3.

\section{Les calcaires dolomitiques}

Dans la partie supérieure de l'horizon des phyllites rubanés, les calcaires dolomitiques forment un niveau repaire constitué de deux bancs discontinus d'épaisseur variable, séparés par des phyllites quartzofeldspathiques. Le plus gros banc, d'une épaisseur de 6 à $8 \mathrm{~m}$, affleure au Sud et au Nord de Kaninelv. La couleur d'altération de ces calcaires est jaune clair ou beige clair.

Si la discontinuité des bancs est due en partie à des déformations tectoniques, comme le souligne Wegmann (1938), "a dolomite horizon which is "boudiné" ", elle correspond aussi à des variations dans les conditions de sédimentation. En effet, une dizaine de mètres en dessous du banc principal se trouve un autre banc, de $5 \mathrm{~m}$ d'épaisseur au maximum, qui s'amincit vers le Sud et passe à des quartzites carbonatés et amphiboliques. De telles variations de faciès s'observent également au Nord, en allant vers Webers Havn.

Dans les calcaires dolomitiques, on peut voir localement un fin rubanement marqué par des lits de couleur gris clair ou beige clair. Le mica, brun, peu coloré, est déjà visible à l'oeil nu. L'amphibole, vert très pâle, est disposée en touffes ou en gerbes allongées parallèlement à la schistosité ou parfois tout à fait obliques. Dans les zones plus riches en minerai de fer (magnétite), la teinte de l'amphibole est souvent plus foncée.

Aú microscope, le carbonate extrêmement granulé, est partiellement recristallisé en petites plages engrainées. L'amphibole, incolore, forme de petits amas de cristaux poecilitiques ou des paquets de fibres très fines. La détermination à la platine universelle des caractères optiques de cette amphibole (tableau $4 \mathrm{~b}$ ) montre que sa composition doit être intermédiaire entre une actinote et une trémolite (WINGHELL, 1959). 
Tableau 4a. Composition minéralogique des calcaires dolomitiques.

\begin{tabular}{|c|c|c|c|}
\hline \multirow[t]{2}{*}{$n^{\circ}$ plaques GGU } & 38704 & 38707 & 38705 \\
\hline & $\%$ & $\%$ & $\%$ \\
\hline Carbonate $\ldots \ldots \ldots \ldots \ldots$ & 84 & 75 & 86 \\
\hline Amphibole.............. & 8 & 11 & 3 \\
\hline Talc . . . . . . . . . . . & 6 & 1 & 8 \\
\hline Mica et chlorite..$\ldots \ldots \ldots$ & 2 & 12 & 3 \\
\hline Minerai de fer........... & $1 / 2$ & 1 & $1 / 2$ \\
\hline
\end{tabular}

Tableau 4b. Caractères optiques des amphiboles dans les roches de la Formation de Taylers Haun.

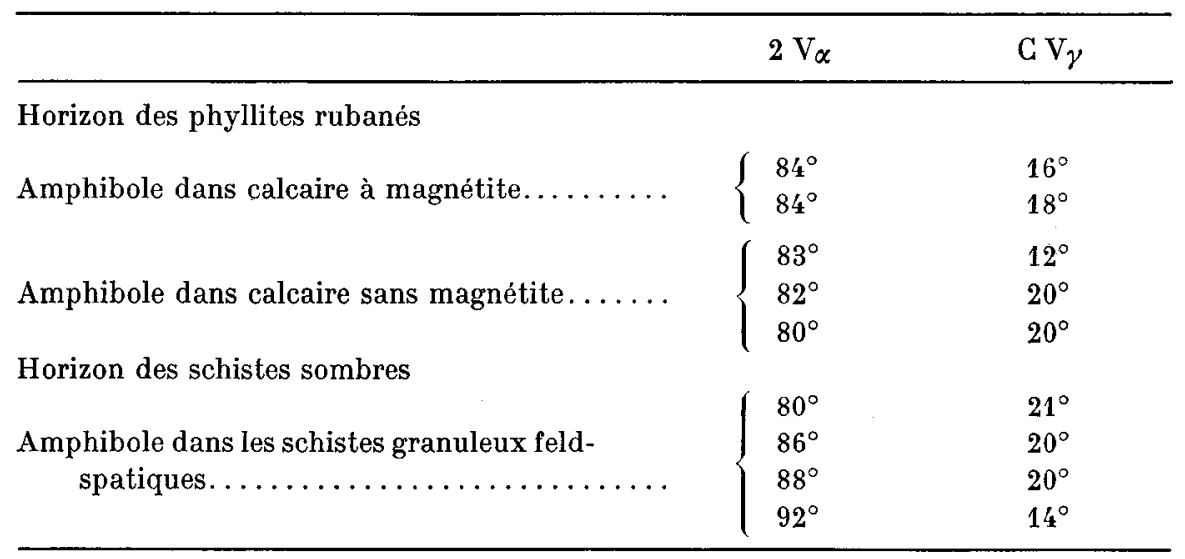

Dans les zones très déformées, les amphiboles sont parfois remplacées par un mica brun, peu coloré.

La composition minéralogique de quelques échantillons de calcaire est donnée dans le tableau $4 \mathrm{a}$.

\section{L'horizon des schistes sombres}

Nous reprendrons ici le terme de schistes sombres ("dark schists") introduit par Wegmann en 1938 pour cet horizon qui surmonte les calcaires dolomitiques. On y distingue plusieurs types de roches: des schistes granuleux, feldspathiques passant latéralement à des phyllites rubanés quartzitiques à biotite. Dans la partie supérieure de cet horizon se trouvent des schistes sombres, parfois très pyriteux et graphiteux ou grenatifères, dans lesquels il y a des intercalations de calcaire et de quartzite dolomitique plus ou moins amphibolique. On y trouve aussi quelques intercalations de roches vertes très déformées (filons-couches).

Les schistes, les quartzites et les calcaires dolomitiques situés sur la côte Nord d'Arsuk $\varnothing$, entre Ikerasârssuk et Qimatut, sont rattachés à cet horizon de schistes sombres. 
Sur l'îlot Kînâlik, au large d'Ikapynt, les schistes pyriteux pincés entre des quartzites et des roches vertes appartiennent probablement aussi à cet horizon de la Formation de Taylers Havn.

\section{Les schistes granuleux feldspathiques}

Ces schistes n'ont été reconnus qu'à Taylers Havn, sur la côte Est de Fiskebenbugt, où ils surmontent directement les calcaires dolomitiques. Sur le petit illot situé à l'Ouest de Ravnenæs, ils sont intercalés entre deux bancs de calcaire. Au Nord de Kaninelv, ils passent à des phyllites quartzitiques sombres.

Ces schistes granuleux sont caractérisés par l'abondance de petits feldspaths blanchâtres, sphériques ou ovoïdes, de 1 à $3 \mathrm{~mm}$ de diamètre, noyés dans une matrice très sériciteuse grisâtre. Ils sont parfois si nombreux qu'ils se touchent et forment des lits blanchâtres de plusieurs millimètres d'épaisseur.

Au microscope, on observe que ces feldspaths sont très cataclasés et même broyés. Ils forment de petits yeux étirés parallèlement aux feuillets de muscovite et de chlorite (planche $2 \mathrm{c}$ ). Les cristaux prismatiques de feldspath sont "microboudinés 》 avec formation de chlorite et de quartz dans les espaces interboudins et dans les fissures dues à la cataclase. Des

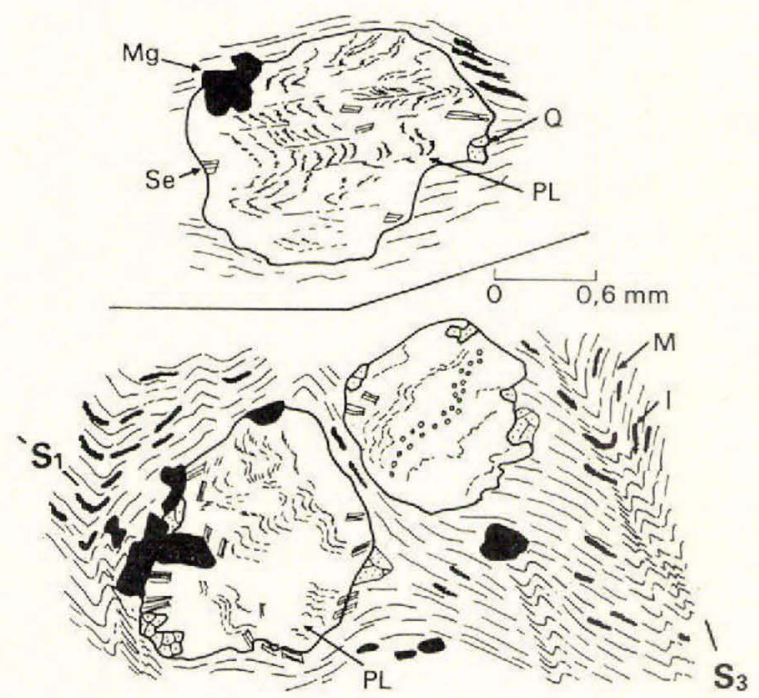

Fig. 11. Pseudo-plis dans les plagioclases calciques (andésine:PL) de l'horizon des schistes granuleux feldspathiques, Fiskebenbugt. Les feuillets de muscovite (M) allongés parallèlement à la direction de la schistosité $\left(S_{1}\right)$ sont déformés par des microplis de la $3^{\mathrm{e}}$ phase accompagnés parfois d'un clivage $S_{3}$. Séricite (Se), quartz (Q) et magnétite $(\mathrm{Mg})$. Les pseudoplis sont marqués par des petites écailles de minerai de fer et des inclusions rondes de quartz. 
déformations postérieures ont encore froissé ces chlorites ou bien ont déplacé les microboudins.

Les grains de feldspath sont généralement chargés d'agrégats de minerai de fer (ilménite?) qui dessinent fréquemment des ondulations microscopiques. Suivant l'orientation de la section mince, ces ondulations de minéraux opaques ressemblent étrangement à de très petits plis (fig. 11). L'examen à la platine universelle montre que ces structures sont dues

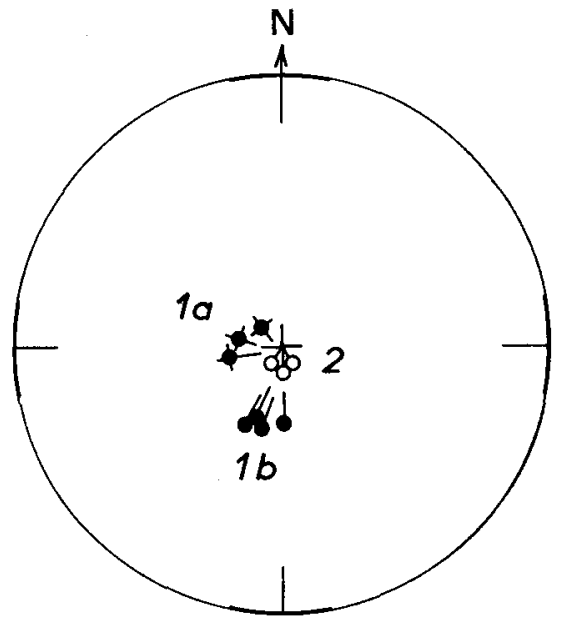

Fig. 12. Orientation des axes des pseudo-plis mesurés à la platine universelle. L'orientation des axes varie d'un plagioclase à l'autre (1a et $1 \mathrm{~b})$. Cette désorientation est liée en partie à la $3^{\mathrm{e}}$ phase (plis 2).

à la disposition de petites écailles de minerai dont certaines se placent dans des zones de discontinuité existant à l'intérieur des cristaux de feldspath (clivage). L'orientation des axes des ondulations varie d'un cristal à l'autre (fig. 12).

Ces «pseudo-plis » pourraient correspondre à des reliques de plissement ancien dont les structures auraient été altérées lors de la cristallisation des feldspaths ("restes polygonaux de plis " souvent observés dans les glaucophanes et les albites).

Les feldspaths sont parfois zonés et leur composition varie dans les limites de l'andésine (détermination à la platine universelle et aux rayons $\mathrm{X}$ ). Ils sont en général fortement séricitisés et saussuritisés. Cette altération souligne encore leur variation de composition car les zones les plus calciques, généralement centrales, sont plus altérées. On observe encore la présence d'albite de néoformation en bordure d'andésine saussuritisée.

La biotite, peu abondante, se forme de préférence sur les plages chloritiques (Harker, 1960). Elle passe, suivant les roches, d'une biotite 


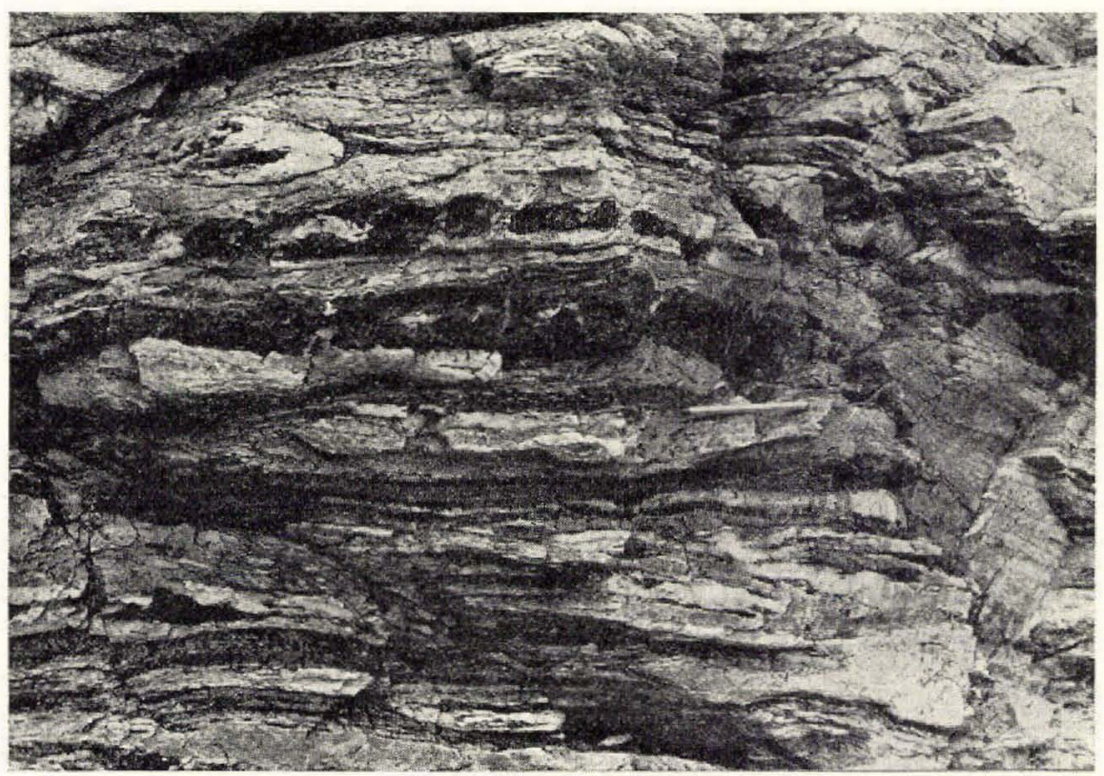

Fig. 13. Boudinage de lits amphiboliques intercalés dans les schistes granuleux feldspathiques. Le feuilletage correspond ici à la schistosité et au clivage. Dans la partie supérieure de la photographie, on reconnaît une charnière de pli contemporain de la schistosité (veine de quartz repliée). Horizon des schistes sombres. Taylers Havn.

verte à une biotite brune. Cette variation doit être liée à la composition chimique des roches dans lesquelles elle a pris naissance.

Le quartz, bien cristallisé, est concentré en lits lenticulaires comme dans les phyllites décrits précédemment. Ces lits peuvent atteindre 2 à $3 \mathrm{~cm}$ d'épaisseur.

L'épidote, plus ou moins idiomorphe, cataclasée, est abondante. Elle est maclée et zonée de la pistacite à la clinozoïsite avec noyau d'orthite. On distingue encore quelques grains arrondis de zircon.

Les résultats de l'analyse planimétrique au compteur de points de 4 échantillons de schistes granuleux sont donnés dans le tableau 5.

Tableau 5. Composition minéralogique des schistes granuleux.

\begin{tabular}{|c|c|c|c|c|}
\hline \multirow[t]{2}{*}{$\mathrm{n}^{\circ}$ plaques GGU } & 38714 & 38713 & 54887 & 38711 \\
\hline & $\%$ & $\%$ & $\%$ & $\%$ \\
\hline Quartz.............. & 20 & 32 & 20 & 35 \\
\hline Feldspath. . . . . . . . . . . . & 32 & 17 & 19 & 21 \\
\hline Muscovite . . . . . . . . . . . & 40 & 19 & 50 & 9 \\
\hline Biotite.............. & rare & 96 & $?$ & 48 \\
\hline Chlorite.............. & 3 & 26 & 2 & 18 \\
\hline Epidote.............. & 2 & 5 & 4 & 15 \\
\hline Minéraux accessoires........ & 3 & 1 & 5 & 2 \\
\hline
\end{tabular}


Vers le haut, les schistes granuleux deviennent plus quartzitiques et renferment des lits amphiboliques vert sombre de 3 à $10 \mathrm{~cm}$ d'épaisseur, discontinus et boudinés (fig. 13).

L'examen microscopique montre que l'amphibole correspond à une trémolite (WINChEI,L 1959) avec $\alpha=$ jaune clair à incolore, $\beta=$ vert et $\gamma=$ vert pâle (tableau 4 a). A l'intérieur des boudins, les amphiboles poecilitiques renferment de nombreuses inclusions de carbonate. Dans la zone externe des boudins, les amphiboles se développent dans un matériel quartzochloriteux riche en épidote et sphène, avec apparition de minuscules taches de biotite. Cette biotitisation ne semble cependant affecter que très peu les amphiboles qui restent pratiquement stables.

\section{Les phyllites quartzitiques à biotite}

Les schistes granuleux passent vers le haut et latéralement vers le Nord à des phyllites quartzitiques gris-noir satinés où la biotite brune est abondante. Au microscope, ces roches sont caractérisées par la disposition lenticulaire de lits micacés et de lits quartzeux.

Ces phyllites portent les traces de plusieurs déformations successives mises en évidence par le plissement en "accordéon " des plans de clivage sur lesquels se trouvent des "intersections» (planche $3 \mathrm{a}$ ).

\section{Le complexe des schistes sombres pyriteux et graphiteux}

Les schistes sombres à biotite et grenat. Les schistes, pyriteux et graphiteux, se distinguent par leur teinte gris-noir due à la présence de substance graphiteuse souvent assez abondante pour tacher les doigts. Leur teinte rouillée, provoquée par l'altération de la pyrite, est caractéristique. La pyrite et le graphite sont répartis irrégulièrement dans les roches. Lors des déformations tectoniques, le graphite joue le rôle de lubrifiant. Dans les zones graphiteuses laminées, les structures sont très complexes.

Dans la région de Taylers Havn, les schistes sombres pyriteux et graphiteux sont principalement localisés sur la presqu'île d'Inugsuk et immédiatoment au Nord de celle-ci, ainsi que sur les côtes Nord et Est de Fiskebenbugt.

Au microscope, ces schistes apparaissent constitués de matériel quartzofeldspathique et micacé dont les proportions varient d'un endroit à l'autre. Le graphite est sous forme de traînées poussiéreuses, tandis que le minerai de fer est en taches ou agrégats de pyrite, hématite, magnétite et ilménite.

Le feldspath, fortement cataclasé, est partiellement recristallisé. Sa composition varie entre l'albite et l'oligoclase. Il est souvent très séricitisé et saussuritisé.

La biotite brune est abondante. Elle contient fréquemment des inclusions d'orthite, des aiguilles de sagénite ou encore des petites taches sombres pléochroïques. Elle se développe en grandes plages poecilitiques ayant jusqu'à $2 \mathrm{~mm}$ de diamètre, se disposant de préférence suivant les plans de clivage de la roche. Mais dans bien des cas les biotites débordent ces zones de discontinuité et les "cicatrisent". Les traînées graphiteuses, qui soulignent les anciennes structures de la roche (schistosité 
et clivage), se retrouvent dans les biotites (fig. 49). Ces biotites ont été ensuite cataclasées lors de la formation de la structure synclinale de Taylers Havn. Elles sont tordues et cassées par le rejeu des plans de clivage qu'elles avaient cicatrisés.

Le grenat, incolore ou rosé (rougeâtre à l'œil nu), a cristallisé sous forme poecilitique (remplacement) en mimant les structures antérieures de la roche telles que les plans $S_{1}$ et $S_{2}$ (fig. 50 ). Il peut atteindre $0,5 \mathrm{~cm}$ de diametre. Comme la biotite, il est postérieur à la formation du clivage. Son développement semble, dans certains cas, plus tardif que celui de la biotite qu'il renferme en inclusion (planche $3 \mathrm{~b}$ ). Le grenat a été déformé lors de la dernière phase de plissement (cataclase, déplacement de fragments et structure cillée des schistes grenatifères). Sa chloritisation est certainement postérieure à cette phase, car des pseudomorphoses chloriteuses sont conservées dans les charnières des petits plis en "accordéon ".

Le quartz est partiellement recristallisé en plages équidimensionnelles et forme des lits lenticulaires. Cette ségrégation est tardive.

Certains schistes rubanés de la pointe méridionale d'Inugsuk sont constitués d'une alternance millimétrique de lits de quartz, séricite, chlorite et biotite et de lits quartzochloriteux à grenats. Ces grenats incolores ne dépassent pas $0,07 \mathrm{~mm}$ de diamètre, mais sont par contre excessivement nombreux, Le rubanement, déjà visible à l'oeil nu, semble d'origine sédimentaire.

Les résultats de l'analyse planimétrique au compteur de points de plusieurs schistes sombres pyriteux et graphiteux sont donnés dans le tableau 6.

Tableau 6. Composition minéralogique des schistes sombres.

\begin{tabular}{|c|c|c|c|c|c|c|}
\hline \multirow[t]{2}{*}{$\mathbf{n}^{\circ}$ plaques GGU } & 48887 & 54882 & 54882 & 56576 & 56579 & 54880 \\
\hline & $\%$ & $\%$ & $\%$ & $\%$ & $\%$ & $\%$ \\
\hline Quartz............... & 4 & 49 & 34 & 19 & 43 & 15 \\
\hline Feldspath............. & - & 2 & 14 & 20 & 6 & 20 \\
\hline Biotite. . . . . . . . . . . & 1 & 8 & 32 & 29 & 12 & 18 \\
\hline Chlorite. . . . . . . . . . . & $>1$ & 3 & 1 & 7 & 2 & 38 \\
\hline Muscovite............ & - & 37 & 19 & 1 & 32 & - \\
\hline Epidote.............. & - & 1 & - & 1 & - & 6 \\
\hline 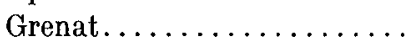 & - & 1 & 1 & - & 4 & - \\
\hline Carbonate............. & - & - & $\ldots$ & 21 & - & 1 \\
\hline Graphite............. & 95 & 1 & 1 & 1 & 1 & 1 \\
\hline Minerai de fer........... & - & 1 & 1 & 2 & 1 & 2 \\
\hline
\end{tabular}

Les intercalations de calcaires dolomitiques. Des calcaires gris, de 1 à 2 mètres de puissance, intercalés dans les schistes graphiteux et pyriteux, sont déformés et boudinés "en grand ». Sur les côtes Nord et Est de Fiskebenbugt, ils sont visibles à plusieurs reprises dans les charnières de plis. Sur la côte Nord-Est d'Inugsuk, ils sont repliés sur eux-mêmes, ce qui augmente considérablement l'épaisseur des affleurements. On en retrouve encore quelques petits lambeaux plus au Sud, pincés dans les schistes pyriteux et graphiteux. 
Leur composition est très semblable à celle des calcaires dolomitiques, plus ou moins amphiboliques, déjà décrits; mais ici la présence de substance graphiteuse est caractéristique.

Les quartzites dolomitiques. Au Nord d'Inugsuk, un banc de 2 à $5 \mathrm{~m}$ d'épaisseur de quartzite carbonaté rubané, de couleur noire et brun roux, forme la limite entre les schistes gris-noir satinés et les schistes plus typiquement pyriteux et graphiteux. Au Nord de Fiskebenbugt, plusieurs petits bancs plissés avec les schistes pyriteux pourraient peut-être appartenir au même niveau, replié plusieurs fois.

Ces quartzites sont constitués de lits quartzocarbonatés jaune-brun et de lits plus quartzitiques gris-noir, de 1 à $2 \mathrm{~cm}$ d'épaisseur. Au microscope, les lits quartzitiques gris-noir sont constitués de quartz en plages engrainées enfermant quelques rares grains d'albite. La pyrite est fréquente. On distingue également un peu de chlorite vert pâle et de minuscules grenats incolores, en partie chloritisés.

Les quartzites. Un banc de quartzite rubané gris-blanc, de 1 à $2 \mathrm{~m}$ d'épaisseur, permet de suivre l'enroulement des plis sur la presqu'île d'Inugsuk. Le quartz forme le constituant principal de ce quartzite, tandis que la chlorite représente le $1 / 5 \mathrm{du}$ volume. Le zircon détritique, l'apatite, le graphite en traînées et le minerai de fer forment les constituants accessoires.

A $50 \mathrm{~m}$ à l'Est de ce banc se trouve un banc de quartzite arkosique à biotite et muscovite. Le feldspath (albite) forme le $1 / 4$ du volume de la roche dans laquelle on note encore la présence de zircon détritique, de grenat idiomorphe, de zoïsite et de pyrite.

Les roches graphiteuses. Ces roches sont généralement très déformées et se présentent sous forme de lentilles tectoniques pincées dans les schistes pyriteux. La teneur en graphite de ces roches varie énormément d'un endroit à l'autre. Un échantillon prélevé dans une lentille graphiteuse contient $95 \%$ de substance opaque (graphite?), $4 \%$ de quartz et $1 \%$ de biotite (analyse planimétrique).

\section{Résumé et conclusion}

L'horizon des schistes sombres est caractérisé par la présence de graphite et de pyrite qui deviennent plus abondants vers le haut de l' horizon.

La biotite et le grenat sont fréquents. Leur cristallisation est postérieure à la formation du clivage ( $2^{\mathrm{e}}$ phase), mais elle est antérieure à la déformation de ce clivage par les petits plis d'entraînement liés à la structure synclinale N-S de Taylers Havn ( $3^{\mathrm{e}}$ phase).

Dans les schistes granuleux feldspathiques, la présence d'andésine s'explique par néoformation due à un métamorphisme. Les possibilités 
d'origine détritique ou volcanique de ces feldspaths calciques doivent être écartées pour plusieurs raisons: absence de tout autre minéral détritique dans les schistes granuleux; la forme prismatique allongée $(2-3 \mathrm{~mm})$ et très étroite de certaines andésines pourrait difficilement être préservée lors d'un transport; enfin, la présence de relique de plissement en inclusion dans ces minéraux implique leur cristallisation dans une roche déjà déformée.

La cristallisation de l'andésine est antérieure à la $3^{\mathrm{e}}$ phase de plissement, qui est responsable de la rotation et de la cataclase de ce minéral. Il n'a pas été possible de placer plus exactement la formation des pseudoplis dans la chronologie, car il n'y a pas de continuité entre les structures reliques et celles du schiste encaissant.

La Formation de Taylers Havn sur la côte Nord d'Arsuk $\varnothing$

Le long de la côte Nord d'Arsuk $\emptyset$ se trouve une bande de roches qui présentent beaucoup d'analogie avec celles de la Formation de Taylers Havn et plus particulièrement avec l'horizon des schistes sombres. Ce sont des schistes rubanés quartzofeldspathiques, à biotite, parfois pyriteux et graphiteux, des calcaires dolomitiques, des phyllites chloritoquartzitiques, des quartzites rubanés ou carbonatés et enfin des roches vertes très déformées correspondant probablement à d'anciens filonscouches. Ces roches sont séparées de l'horizon des quartzites dolomitiques par le détroit d'Ikerasârssuk qui est probablement situé sur une des nombreuses zones mylonitiques recoupant la côte Nord d'Arsuk $\varnothing$.

Dans toute la région de la côte Nord d'Arsuk $\emptyset$, le faciès originel des roches est très souvent modifié par des venues tardives de roches carbonatées, accompagnées de minéralisation de fer, qui se sont infiltrées dans les zones écrasées et mylonitiques et imprègnent les roches encaissantes (p. 118).

\section{Les schistes quartzofeldspathiques à biotite}

Ces schistes, visibles à l'Ouest de Pâkitsúnguaq, sont en général très écrasés. Les roches les moins déformées sont caractérisées par une alternance millimétrique de lits chloriteux à biotite et de lits quartzofeldspathiques parfois carbonatés. La biotite est déjà visible à l'oeil nu.

Au microscope, on observe une granulation très forte du matériel quartzofeldspathique. La chlorite est en petites écailles disposées parallèlement à la schistosité. La biotite brune, poecilitique, se développe de préférence dans les lits chloriteux. Cette biotite est cataclasée et un peu chloritisée. Le quartz, recristallisé " lamellairement", est concentré en lits lenticulaires parallèles à la schistosité. Le carbonate contenu dans ces schistes est probablement lié aux venues tardives de roches carbonatées. 


\section{Les calcaires dolomitiques, les phyllites et les quartzites}

Les calcaires dolomitiques se trouvent à l'Ouest de Pâkitsúnguaq. Ils sont en bancs plissés et boudinés, lacérés de veinules de quartz (WEGMANN, 1938, fig. 3). Ce quartz provient sans doute d'une ségrégation siliceuse analogue à celle observée dans les phyllites à Taylers Havn. La trémolite est rare ou absente. Le minerai de fer est sous forme de pyrite idiomorphe, déjà visible à l'oeil nu.

Les phyllites chloritoquartzitiques se trouvent principalement à l' Ouest de Pâkitsúnguaq. Leur couleur, souvent gris-noir, est due à la présence de graphite.

Sous le microscope, la biotite brune renferme en inclusion de nombreuses trainées graphiteuses. Le plagioclase, dont la composition varie entre l'albite et l'oligoclase, est cataclasé et peu abondant. Il renferme aussi des inclusions graphiteuses disposées en traînées.

Les calcschistes chloriteux sont composés de matériel quartzofeldspathique, de chlorite et de carbonate, ces trois constituants étant à peu près en égale quantité.

Les quartzites rubanés graphiteux de Pâkitsúnguaq sont formés d'une alternance millimétrique de lits de quartz et de lits quartzographiteux noirs. La granulation du quartz est toujours plus forte dans les lits quartzographiteux que dans les lits purement quartzitiques.

\section{Conclusion}

La Formation de Taylers Havn est composée de sédiments semipélitiques devenant de plus en plus fins et pélitiques vers le haut. Elle est caractérisée par la présence de niveaux calcaires peu épais. On observe quelques variations latérales brusques de faciès, notamment dans les bancs de calcaire dolomitique passant à des quartzites carbonatés.

Les roches quartzofeldspathiques sont abondantes dans toute la formation. L'origine détritique d'une partie du feldspath contenu dans ces roches nous paraît vraisemblable à la base de l'horizon des quartzites. Mais généralement, ce minéral est néoformé.

Le litage originel des roches n'est pratiquement plus visible à cause des fortes déformations et recristallisations subies par les sédiments. En effet, trois phases de déformation successives ont été reconnues dans le secteur de Taylers Havn. Elles correspondent respectivement à l'apparition de la schistosité, au développement du clivage (recoupant la schistosité) et enfin à la déformation de ce clivage lors de la formation du synclinal N-S de Taylers Havn.

L'épaisseur maximum observable de la Formation de Taylers Havn est de 350 à $400 \mathrm{~m}$. L'épaisseur réelle est sans doute inférieure du fait des 
redoublements des couches de phyllites et de schistes qui la composent principalement. La partie supérieure de cette formation n'est pas connue.

Les reconstructions structurales de la région d'Arsuk $\varnothing$ permettent de penser que le fjord d'Ikerasârssûp kujatâ large de $2 \mathrm{~km}$, séparant Taylers Havn de la côte orientale d'Arsuk $\varnothing$, est occupé par une série ayant au moins $500 \mathrm{~m}$ d'épaisseur. Cette série pourrait être la formation semi-pélitique d'Inugsugtût caractéristique à Storø, à $7 \mathrm{~km}$ au SudOuest d'Arsuk Ø.

\section{Situation}

\section{La Formation d'Inugsugtût}

La Formation semi-pélitique d'Inugsugtût $\dagger$ n'a été reconnue que sur l'île Storø. Dans la région d'Arsuk $\varnothing$, sa présence est tout à fait hypothétique. Comme nous l'avons vu, elle pourrait occuper une partie du fjord d'Ikerasârssûp kujatâ et combler ainsi le hiatus existant entre la Formation de Taylers Havn et celle d'Isua.

Nous présentons ici la succession lithologique observée dans la partie Sud-Est de Storø. Les roches sédimentaires ont été divisées en deux formations, séparées par un filon-couche gabbro-dioritique de $300 \mathrm{~m} \mathrm{~d}$ ' épaisseur environ. La formation supérieure doit correspondre à celle d'Isua, la formation inférieure a été appelée Formation d'Inugsugtût.

La base de la Formation d'Inugsugtût n'est pas connue. Les roches sédimentaires qui af fleurent sur les îlots Inugsugtût, à $2 \mathrm{~km}$ à l'Est de Storø appartiennent à la partie la plus basse observable de cette formation (Froidevaux \& Muller, 1958).

Succession lithologique observée dans la partie supérieure de la Formation d'Inugsugtût à Storø

De haut en bas, on a:

filon-couche de gabbro-diorite (300 $\mathrm{m}$ environ)

schistes pyriteux

quartzites massifs gris-blanc

phyllites quartzitiques gris clair tachetés*, par endroit schistes très pyriteux

quartzites feuilletés sériciteux

phyllites rubanés quartzosériciteux tachetés *

quartzites feuilletés sériciteux

quartzites massifs

phyllites rubanés gris ardoise, parfois pyriteux et tachetés*

$\dagger$ Cette formation avait été appelée "Levant Formation" par Bondesen (1970, p. 143).

* Ces taches correspondent à des minéraux de métamorphisme de contact (intrusion granitique de Storø). 
phyllites granuleux

filon-couche de gabbro-diorite de 10 à $100 \mathrm{~m}$ d'épaisseur

phyllites rubanés quartzitiques

quartzites sériciteux rubanés et schistes pyriteux

quartzites rubanés tachetés*

Sur la côte Sud de Storø, la succession lithologique est plus difficile à établir, car les roches sont très plissées. On note principalement des grès sériciteux rubanés (planche $3 \mathrm{c}$ ). Sur les îlots Inugsugtût, les roches sédimentaires sont représentées principalement par des phyllites quartzitiques rubanés, des quartzites massifs ou rubanés, avec structures sédimentaires syngénétiques (glissements sous-marins, coulées de boue, granoclassement), ainsi que l'avait déjà observé WeGmann (1938, p. 21).

\section{Résumé}

Les roches quartzitiques constituent la plus grande partie de la Formation d'Inugsugtût. Les structures sédimentaires syngénétiques y sont généralement conservées.

L'épaisseur des roches sédimentaires de la partie observable de cette formation est évaluée à 400 ou 500 mètres.

\section{La Formation d'Isua}

La Formation d'Isua sur Arsuk $\varnothing$

Sur Arsuk $\varnothing$, la Formation d'Isua est composée de phyllites quartzitiques ou quartzofeldspathiques rubanés surmontés d'un horizon de phyllites pyriteux contenant des intercalations discontinues et lenticulaires de quartzite très finement cristallisé (chert ?).

Sur la côte Nord d'Arsuk $\varnothing$, entre Blålershavn et Niaqornarssuaq, les roches de la Formation d'Isua chevauchent les schistes sombres rubanés graphitopyriteux de la Formation de Taylers Havn dans lesquels sont pincés de gros filons-couches de gabbro-diorite.

Sur le versant Nord-Est de l'île, dans la région de Skælsiden, les phyllites rubanés et les phyllites pyriteux d'Isua, ainsi que les masses de roches vertes intercalées, sont très déformés (Wegmann, 1938, fig. 26). Les structures sont constituées par des plis d'axe sensiblement E-W, compliqués par des chevauchements (fig. 41, p. 103).

Sur la côte Nord-Ouest de l'île, entre Kæruldkyst et Inugsukasik, les phyllites rubanés viennent en contact anormal avec des schistes verts localement pyriteux et des roches vertes massives de l'unité de transition du Groupe volcanique d'Arsuk. 


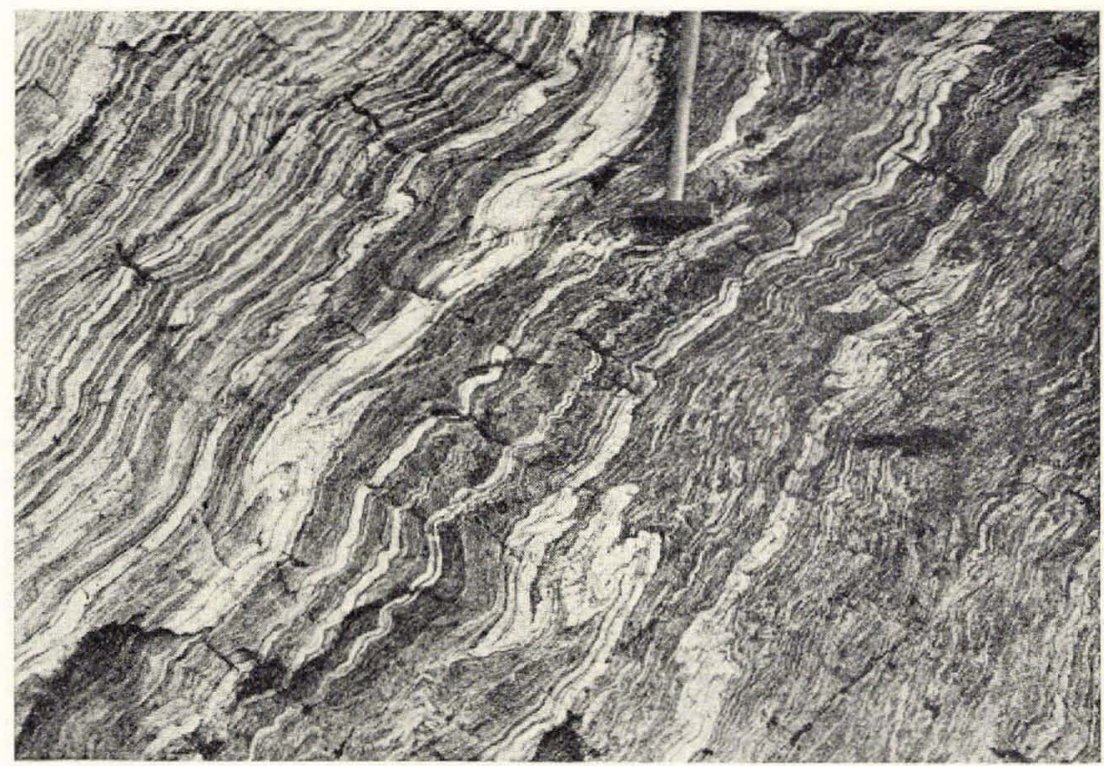

Fig. 14. Phyllites rubanés et plissés de la Formation d'Isua. Inugsukasik, côte Nord d'Arsuk $\varnothing$. Le rubanement correspond ici à la schistosité. Celle-ci est déformée par des plis de style très souple.

\section{Les phyllites rubanés sombres}

Les phyllites rubanés les plus typiques sont visibles dans la région d'Isua, sur la côte orientale d'Arsuk Ø et à Inugsukasik, sur la côte Nord-Ouest. Ces phyllites sont constitués d'une alternance de lits leucocrates quartzeux ou quartzofeldspathiques et de lits sombres phylliteux. Par endroit, les roches sont très phylliteuses et de teinte gris-noir assez uniforme. Cette teinte sombre est due à la présence de substance graphiteuse qui, même en quantité très faible, suffit à colorer les roches. Le minerai de fer est sous forme d'ilménite ou de pyrite dont l'altération confère une teinte rouille aux roches qui la contiennent en quantité suf fisante.

Le rubanement visible sur le terrain correspond généralement à la schistosité (fig. 14). Le litage originel des sédiments n'est visible que dans les secteurs où les charnières de plis synchrones de la schistosité n'ont pas été déformées ultérieurement (fig. 38, p. 99).

L'examen microscopique d'un phyllite rubané affecté de schistosité (planche 4a) montre que les lits leucocrates sont constitués soit de quartz, soit de feldspath et de quartz avec un peu de chlorite et de séricite. Les colorations au cobaltonitrite révèlent une absence quasi totale de feldspath potassique. Le plagioclase, dont la composition varie entre l'albite et l'oligoclase acide, est en très petits grains. Suivant la teneur en graphite de la roche, ce minéral peut ètre chargé d'inclusions de particules graphiteuses.

Les lits sombres phylliteux sont constitués de chlorite et de séricite en toutes petites paillettes disposées parallèlement à la schistosité. Les granules d'ilménite, 
plus ou moins leucoxénisés, s'allongent aussi suivant cette schistosité. Les autres constituants de la roche sont le zircon (détritique) et la tourmaline tardive, auxquels s'ajoute rarement un peu de carbonate.

La biotite est peu fréquente dans toutes les roches appartenant à la Formation d'Isua. Néanmoins, dans la région Nord de Skælsiden, on observe des phyllites chloritoquartzeux carbonatés à ilménite et biotite et des phyllites quartzitiques sombres à biotite et grenat.

L'examen microscopique de ces dernières roches montre que le quartz, en très petits cristaux, a tendance à s'allonger parallèlement à la schistosité, de même que la chlorite et la muscovite. La biotite brune, en petits feuillets, se dispose de la même manière. Elle est répartie suivant d'anciens lits qui peuvent correspondre à la stratification. Ces lits sont plissés et la schistosité est parallèle au plan axial des plis. Les grenats, incolores, xénomorphes, contiennent des inclusions de quartz. Leur structure est hélicitique. Comme à Taylers Havn, leur chloritisation est postérieure à la déformation principale de la roche.

Dans les roches de la Formation d'Isua, la dimension des cristaux de quartz et de feldspath est toujours très petite et n'excède que rarement $0,1 \mathrm{~mm}$ de diamètre. Les feldspaths à contours lobés peuvent avoir un diamètre double. Les minéraux phylliteux sont aussi extrêmement petits. Cette taille s'explique par la granulation des minéraux liés à des mouvements tectoniques et par le faible degré de recristallisation des constituants de la roche.

Les effets variables de la granulation sont déjà visibles dans un même échantillon. Le matériel quartzofeldspathique est beaucoup plus granulé dans les lits riches en substance graphiteuse ou en minéraux phylliteux. D'un lit à l'autre, la taille des minéraux quartzeux ou feldspathiques peut doubler. Ce phénomène a déjà été observé dans les quartzites rubanés graphiteux de Pâkitsúnguaq appartenant à la Formation de Taylers Havn.

Dans certains phyllites, on observe un fort développement de séricite qui parvient à voiler considérablement les structures de la roche. Cette séricite, en toutes petites paillettes, provient sans doute de la transformation de feldspath complètement pulvérisé. Dans ces roches, le quartz est en grains extrêmement petits, de diamètre moyen voisin de $0,02 \mathrm{~mm}$.

De puissantes masses de roches vertes sont intercalées dans les phyllites de la Formation d'Isua, ainsi qu'on peut le voir sur la carte géologique au 1:20000 d'Arsuk Ø. Ces masses, correspondant probablement à d'anciens filons-couches, seront étudiées avec le matériel volcanique du Groupe d'Arsuk.

\section{L'horizon des phyllites pyriteux avec cherts lenticulaires}

Les phyllites rubanés sombres sont surmontés par un horizon d'une dizaine de mètres d'épaisseur, constitué de phyllites pyriteux contenant 
quelques minces lits quartzitiques lenticulaires (de $10 \mathrm{~cm}$ d'épaisseur au maximum), formés de quartz en grains minuscules, qui pourraient correspondre à des cherts complètement recristallisés (p. 79).

Cet horizon se poursuit tout le long de la côte orientale d'Arsuk $\varnothing$. $\mathrm{Au}$ Nord-Est, à Skælsiden et sur le versant Nord de l'île, il est très déformé et pincé entre des schistes chloritoamphiboliques appartenant au groupe volcanique. Sur la côte Nord-Ouest, entre Kæruldkyst et Inugsukasik, cet horizon disparaît. Cette disparition est probablement due à la tectonique car, à cet endroit, les phyllites sombres rubanés sont en contact tectonique anormal avec les schistes verts du groupe volcanique.

Un autre horizon du même type forme la limite entre les unités de transition et inférieure du groupe volcanique. Il est caractérisé, dans la région occidentale d'Arsuk $\emptyset$ et sur l'îlot Mitdlûvfik, par des quartzites rubanés disposés en petites lentilles, en nodules ou en "plaques " (fig. 27 , p. 80).

Les deux niveaux de phyllites pyriteux avec cherts lenticulaires visibles sur Arsuk $\varnothing$ ne constituent plus qu'un seul niveau à Storø, car l'unité volcanique de transition s'amincit en direction de l'Ouest et n'existe pas sur cette île.

\section{La Formation d'Isua à Storø}

A Storø, nous avons fait débuter la Formation d'Isua au-dessus du filon-couche gabbro-dioritique de $300 \mathrm{~m}$ d'épaisseur qui affleure sur la côte Est de l'île. A la base de la formation se trouvent des quartzites massifs ou rubanés qui ressemblent beaucoup à ceux de la Formation d' Inugsugtût. $\mathrm{Au}$ sommet se trouve un horizon de phyllites pyriteux avec cherts lenticulaires qui est surmonté, comme sur Arsuk $\emptyset$, par des roches volcaniques appartenant au Groupe d'Arsuk.

La succession lithologique observée le long de la côte orientale de Storø, entre le gros filon-couche et les pillows du groupe volcanique, est représentée sur la fig. 15. Cette succession est incomplète à la base car les roches sont recouvertes par des dépôts quaternaires (Froidevaux \& MulLeR, 1958).

\section{Conclusion}

La Formation d'Isua est constituée principalement de sédiments semi-pélitiques caractérisés par la présence, uniforme mais en faible quantité, de substance graphiteuse. Son épaisseur maximale observable est d'une centaine de mètres. La base de la Formation d'Isua n'est pas connue sur Arsuk Ø. Par contre, à Storø, cette formation passe en transition à la Formation d'Inugsugtût.

Les cherts, situés à la partie supérieure de la Formation d'Isua, terminent le Groupe sédimentaire d'Ikerasârssuk. Ces cherts comme nous 
$3 \mathrm{~m}$ "quartzitic slabs" avec intercalations de phyllites gris-noir

$3 \mathrm{~m}$ phyllites gris ardoise rubanés granuleux

$1 \mathrm{~m}$ quartzites phylliteux verdâtres rubanés

$3 \mathrm{~m}$ quartzites verdâtres massifs

$12 \mathrm{~m}$ phyllites rubanés verdâtres et gris

$1,5 \mathrm{~m}$ filon-couche de gabbro-diorite

$5 \mathrm{~m}$ phyllites rubanés sombres tachetés ${ }^{(1)}$

$3 \mathrm{~m}$ quartzites un peu sériciteux rubanés avec glissements sous-marins - quartzites tachetés à la base ${ }^{(1)}$

$6 \mathrm{~m}$ phyllites rubanés tachetés ${ }^{(1)}$

$8 \mathrm{~m}$ quartzites massifs tachetés(1)

$10 \mathrm{~m}$ phyllites gris rubanés tachetés ${ }^{(1)}$

$1 \mathrm{~m} \quad$ quartzites massifs

$14 \mathrm{~m}$ phyllites quartzitiques tachetés ${ }^{(1)}$

quartzites massifs verts et blancs

non exposé

filon-couche de gabbro-diorite (300 m d'épaisseur)

(1) Minéraux de métamorphisme de contact (granite intrusif de Storø)

Fig. 15. Coupe lithologique de la partie supérieure de la Formation d'Isua sur la côte orientale de Store. 
le verrons, pourraient être déjà liés génétiquement à l'activité volcanique qui succède au dépôt des sédiments et qui donnera naissance au Groupe volcanique d'Arsuk.

\section{Le Groupe volcanique d'Arsuk}

Le Groupe volcanique d'Arsuk* surmonte le Groupe sédimentaire d'Ikerasârssuk. Sur Arsuk $\varnothing$, son épaisseur maximale observable est de $4200 \mathrm{~m}$. Il est composé principalement de matériel volcanique avec quelques intercalations continues ou lenticulaires de matériel sédimentaire. Ce groupe a été divisé en quatre unités qui sont de haut en bas:

\section{Groupe volcanique d'Arsuk unité moyenne unité inférieure \\ unité de transition}

La division en quatre unités est basée sur des critères de terrain comme la forme, la couleur et la nature des principaux produits volcaniques. Nous devons à Wegmann (1938) la première description des roches composant cette série volcanique qu'il a appelé "groupe d'Arsuk ».

\section{Coupe lithologique}

La coupe lithologique la plus intéressante à travers le Groupe volcanique d'Arsuk est visible en effectuant une traversée Nord-Sud d'Arsuk, entre Kæruldkyst situé sur la côte Nord, le lac $490 \mathrm{~m}$ et le torrent qui rejoint la côte Sud (fig. 16). De bas en haut on a la succession suivante:

Kæruldkyst phyllites pyriteux et graphiteux plus ou moins rubanés apparteAlt. $0 \mathrm{~m}$ nant à la Formation sédimentaire d'Isua

Unité de transition du Groupe volcanique d'Arsuk Epaisseur: $400 \mathrm{~m}$ environ

Blåbærelv schistes actinolitiques

Alt. $5 \mathrm{~m} \quad$ roches gabbro-dioritiques massives, à grain fin (p. 70), recoupées localement par des zones de laminage et de cisaillement schistes actinolitiques

Alt. $20 \mathrm{~m}$ roches gabbro-dioritiques massives grain grossier coulée de pillows bréchiques et isolés de 3 à $4 \mathrm{~m}$ d'épaisseur (p. 66).

roches gabbro-dioritiques schisteuses et plissotées par endroit

Alt. $35 \mathrm{~m}$ horizon de roche quartzitique gris-blanc de 3 à $4 \mathrm{~m}$ d'épaisseur passant vers l'Ouest à des phyllites pyriteux avec cherts lenticulaires (p. 79)

* Ce Groupe d'Arsuk avait été appelé "Arsûta Formation» par Bondesen (1970, p. 143). 


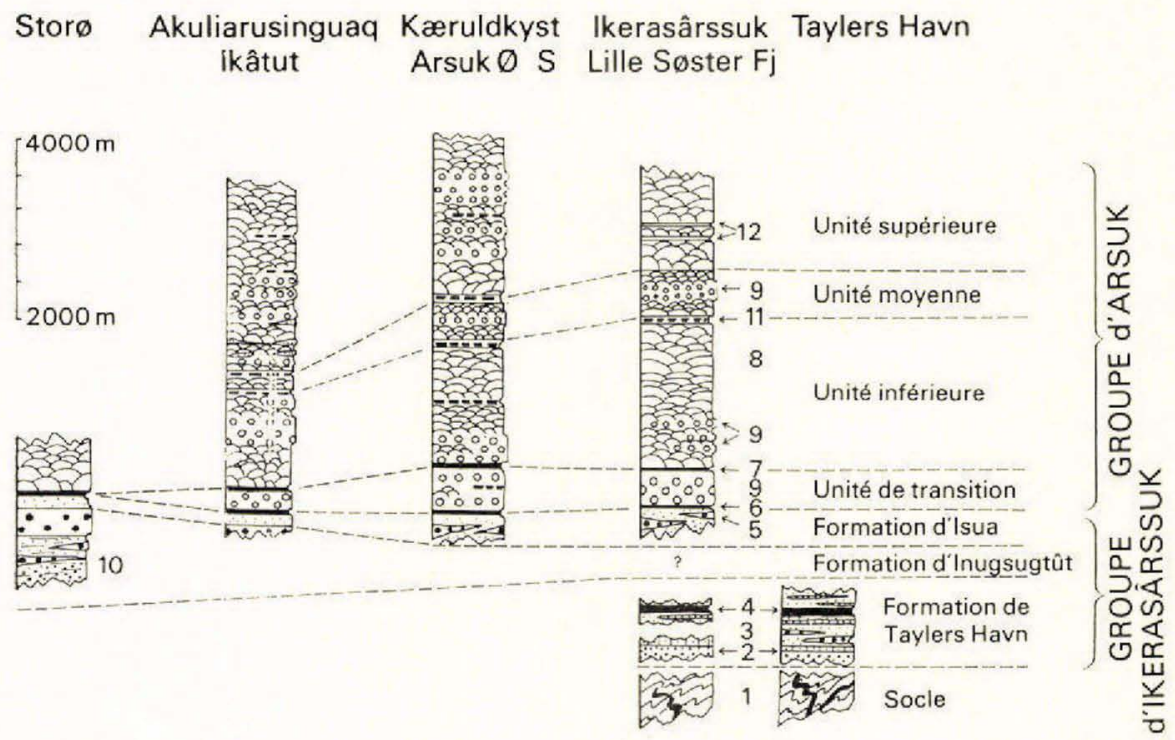

Fig. 16. Coupes lithologiques à Storø et Arsuk $\varnothing$. 1: gneiss prékétilidiens. 2: quartzites massifs et quartzites dolomitiques. 3 : phyllites rubanés et calcaires dolomitiques. 4: schistes pyriteux et graphiteux et schistes sombres. 5: filons-couches de gabbrodiorite. 6: phyllites sombres et pyriteux et cherts lenticulaires. $7:$ cherts lenticulaires (quartzitic slabs). 8: pillows. 9: Laves massives (gabbro-diorites). 10: phyllites et quartzites. 11: tufs, lapillis et brèches volcaniques. 12: ultrabasites.

Alt. $35 \mathrm{~m}$

Alt. $40 \mathrm{~m}$

Torrent du lac $490 \mathrm{~m}$

Alt. $125 \mathrm{~m}$

Alt. $250 \mathrm{~m}$

Alt. $420 \mathrm{~m}$

Lac $490 \mathrm{~m}$

Rive W du lac $490 \mathrm{~m}$

\section{Unité inférieure du Groupe d'Arsuk} Epaisseur: $1500 \mathrm{~m}$ environ

roches gabbro-dioritiques massives, à grain fin ou moyen, localement schisteuses, de $400 \mathrm{~m}$ d'épaisseur coulées de pillow-lavas de type ellipsoïdal (p. 51)

coulées de pillows bréchiques et isolés (3 à $7 \mathrm{~m}$ d'épaisseur). Vers l'Ouest ce matériel passe à des brèches volcaniques détritiques coulées de pillow-lavas de type ellipsoïdal

coulée de pillows bréchiques et brèches volcaniques détritiques (2 à 3 m d'épaisseur)

coulées de pillows de type ellipsoïdal avec quelques masses de roches gabbro-dioritiques à grain fin

coulée de pillows bréchiques et isolés (1 à $5 \mathrm{~m}$ d'épaisseur)

Unité moyenne du Groupe d'Arsuk

Epaisseur $500 \mathrm{~m}$ environ

schistes actinolitiques pyriteux

coulées de pillow-lavas de type amiboïde (p. 56). On note aussi la présence de pillows de type ellipsoïdal particulièrement aplatis et allongés. Dans ces coulées, il y a des lentilles de chert pyriteux de 1 à $1,5 \mathrm{~m}$ d'épaisseur 
roches gabbro-dioritiques massives, à grain fin ou moyen, de $80 \mathrm{~m}$ d'épaisseur environ

Rive E du lac coulées de pillows amiboïdes et ellipsoïdaux avec des intercala$490 \mathrm{~m}$ tions de roches quartzitiques lenticulaires de 0,2 à $2 \mathrm{~m}$ d'épaisseur

\author{
Unité supérieure du Groupe d'Arsuk \\ Epaisseur visible: $1600 \mathrm{~m}$
}

Extrémité S du lac coulées de pillows du type ellipsoïdal

$490 \mathrm{~m} \quad$ coulées de pillows bréchiques de 1 à $2 \mathrm{~m}$ d'épaisseur

coulées de pillows ellipsoïdaux, avec quelques intercalations de cherts pyriteux lenticulaires. On note localement quelques brèches volcaniques

Col au $\mathrm{S}$ du lac roches gabbro-dioritiques massives de $20 \mathrm{~m}$ d'épaisseur $490 \mathrm{~m}$

Ravin descendant sur le flanc Est du ravin, à l'altitude $700 \mathrm{~m}$, se trouve une masse vers la côte $\mathbf{S}$ de roche ultrabasique intercalée dans les coulées de pillows

Torrent

Alt. $500 \mathrm{~m}$ coulées de pillows ellipsoïdaux alternant avec des roches gabbro-

Alt. $430 \mathrm{~m}$ dioritiques à grain fin

Alt. $300 \mathrm{~m}$ coulées de pillows

alternance de coulées de pillows et de roches gabbro-dioritiques massives à grain plus ou moins fin

Alt. $270 \mathrm{~m}$ roches gabbro-dioritiques massives. Vers l'Ouest ces roches sont très laminées

Alt. $250 \mathrm{~m}$ coulées de pillows ellipsoïdaux

Côte $\mathrm{S}$

Alt. $20 \mathrm{~m}$

coulées de pillows avec quelques intercalations lenticulaires de chert de 1 à $2 \mathrm{~m}$ d'épaisseur

La partie supérieure de cette succession n'est pas connue, car elle est recouverte par la mer.

Entre Kæruldkyst et la côte Sud d'Arsuk $\varnothing$, les coulées de pillowlavas représentent $70 \%$ de l'épaisseur totale du Groupe volcanique d'Arsuk, soit $2900 \mathrm{~m}$ environ. Les coulées de laves massives et les filonscouches gabbro-dioritiques en représentent $29 \%$ (soit $1200 \mathrm{~m}$ environ). Les coulées de pillows bréchiques et le matériel sédimentaire n'en constituent que $1 \%$ (entre 50 et $100 \mathrm{~m}$ d'épaisseur). Des coupes faites en d'autres endroits d'Arsuk $\varnothing$ donnent des pourcentages semblables.

\title{
Le matériel volcanique
}

Parmi les produits d'origine volcanique on distingue, presque dans chaque unité, plusieurs types morphologiques qui sont:

Les coulées de lave avec structures en pillows, les coulées de pillows bréchiques ou isolés,

les lapillis et les tufs,

les laves massives associées aux coulées de pillows. 


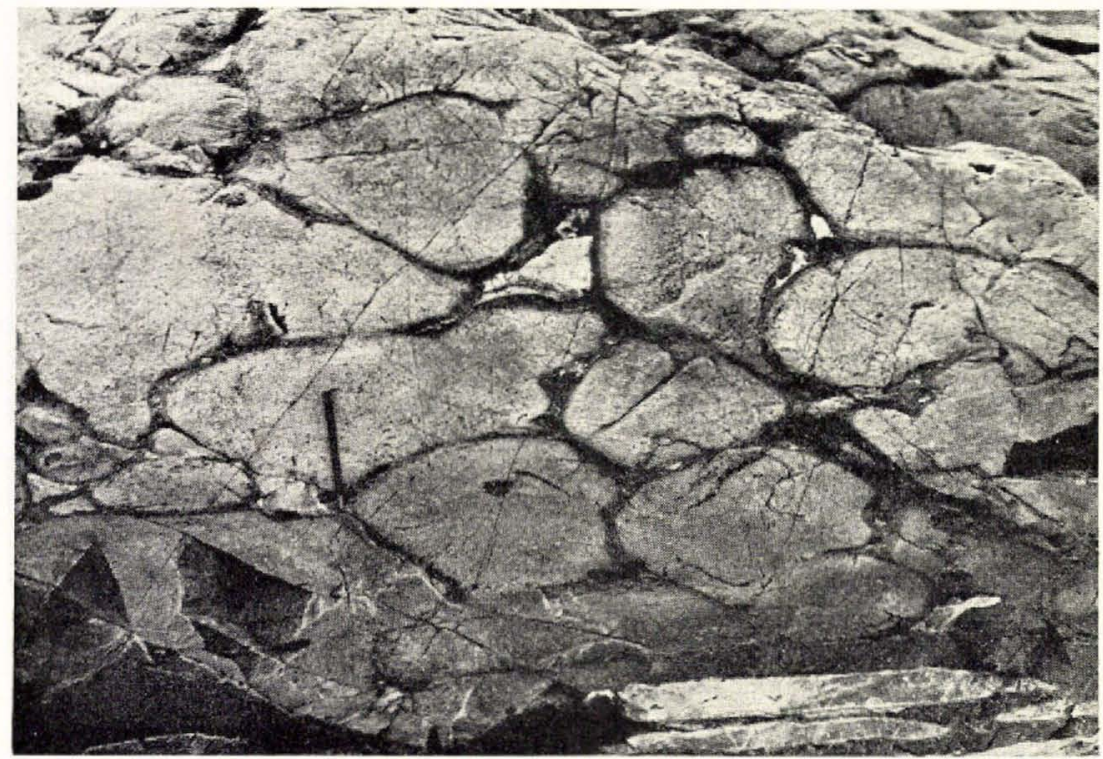

Fig. 17. Entassement de pillow-lavas du type ellipsoïdal, coupés perpendiculairement à leur grand axe. Unité volcanique supérieure à fkátut, sur la côte Ouest d'Arsuk $\varnothing$. La base des pillows est indiquée par la pointe du crayon. Remplissage quartzitique dans les interstices des pillows. Certains pillows communiquent entre eux (partie supérieure de la photographie). Cavités allongées remplies de quartz dans un grand pillow (partie inférieure de la photographie).

Les coulées de lave avec structures en pillows Caractères morphologiques des pillows

Les coulées de lave avec structures en pillow-lavas (ou en coussins) constituent la plus grande partie de l'édifice volcanique. On distingue deux types morphologiques qui sont:

les pillow-lavas "ellipsoïdaux"

les pillow-lavas " amiboïdes".

Les pillow-lavas ellipsoüdaux. Dans cette catégorie sont rassemblés tous les pillows dont la forme se rapproche de celle d'un ellipsoïde plus ou moins aplati. Des pillows de ce type se trouvent dans les unités inférieure et supérieure du Groupe volcanique d'Arsuk. Dans l'unité moyenne, on rencontre également des pillows ellipsoïdaux, mais ces derniers sont très déformés et particulièrement aplatis et allongés.

La dissymétrie observée entre le sommet et la base des pillows permet de définir la polarité des coulées. La partie supérieure des pillows est généralement convexe alors que la base, moulant les irrégularités des pillows précédemment formés et déjà refroidis, présente des formes assez variables (fig. 17). Le modelé de cette base varie suivant la position et la 
grandeur des nouveaux pillows par rapport à ceux sur lesquels ils se déposent. Dans une même coulée, on trouve habituellement quelques-unes des formes suivantes:

base à pédoncule simple allongé (le pillow supérieur repose sur deux pillows disposés côte à côte),

base à pédoncules multiples (le pillow supérieur recouvre plusieurs pillows de diamètre plus petit),

base plane (le pillow supérieur est disposé sur un large pillow peu convexe),

base concave (le pillow supérieur est plus grand que le pillow sur lequel il repose).

Ces formes peuvent se combiner entre elles et on observe toutes les transitions. Les "balloon-type" et "bun-type" de M. E. Wilson (1942) se rattachent au $2^{\mathrm{e}}$ et $3^{\mathrm{e}}$ type. Les grands plans de diaclases recoupant un entassement de pillows donnent une idée assez inexacte des formes spatiales des pillows. Ceux-ci étant coupés en plusieurs endroits par un même plan, les tranches de pillows ainsi exposées ont naturellement des formes différentes. Il est donc indispensable de pouvoir examiner les pillows dans les trois dimensions avant d'en entreprendre la description.

Dans les coulées de pillows ellipsoïdaux non déformés, le petit axe de l'ellipsoïde est perpendiculaire à la surface de la coulée, tandis que l'axe moyen et le grand axe sont plus ou moins parallèles à celle-ci (Vuagnat \& Pustaszeri, 1965). Cette surface, que nous avons utilisée comme plan de "stratification", est plus ou moins parallèle à la base plane ou légèrement concave des pillows des $3^{\mathrm{e}}$ et $4^{\mathrm{e}}$ types. Dans le cas de pillows à base pédonculée, elle est perpendiculaire aux pédoncules.

Sur Arsuk $\varnothing$, les pillows ellipsoidaux sont fréquemment très aplatis. A l'aplatissement syngénétique, dû à l'étalement du pillow par gravité ainsi que la charge des pillows sus-jacents, se superpose un aplatissement et un allongement, parfois très importants, dus aux déformations tectoniques.

La dimension des pillows varie beaucoup, comme l'a noté Wegman N $(1938$, p. 24), «from a decimeter to the size of a large bag 》. Dans la région montagneuse, au Nord du lac $490 \mathrm{~m}$, certains pillows ont $2 \mathrm{~m}$ de long sur $0,5 \mathrm{~m}$ d'épaisseur. Au col $730 \mathrm{~m}$, à l'Est de Storpuklen, quelques individus mesurent jusqu'à $5 \mathrm{~m}$ de long sur $0,55 \mathrm{~m}$ d'épaisseur.

La surface des pillows est généralement lisse. Dans quelques cas, leur croûte supérieure est recoupée par un réseau de très fines craquelures dues à l'éclatement partiel des pillows.

Les structures internes des pillows ellipsö̈daux. Nous avons adopté la terminologie définie par VuAGNaT $(1946,1948,1949,1951)$ dans plusieurs de ses travaux sur les pillow-lavas. 
L'intérieur des pillows est caractérisé par deux types de structures bien mises en évidence par les sections polies:

une structure concentrique due à la fois à une variation de composition et de texture de la roche s'accompagnant de changements de teinte (planches $7 \mathrm{a}$ et $8 \mathrm{~b}$ ),

une structure radiale qui correspond à la fissuration du pillow lors de son refroidissement (fentes de retrait, planche 6 et fig. 18).

Les fentes de retrait sont sinueuses et bordées d'un liseré noir (ilménite et leucoxène). On ne doit pas les confondre avec les fissures tardives, en général plus rectilignes, et souvent accompagnées de remplissage de quartz et de calcite (planches $7 \mathbf{a}$ et $8 \mathbf{a}$ ).

Dans les pillows sphériques ou ellipsoïdaux de petit diamètre, les fentes de retrait sont disposées plus ou moins radialement. Ces fissures, dont les lèvres ont parfois un écartement de $0,5 \mathrm{~mm}$ dans la zone centrale, deviennent de plus en plus fines en allant vers la croute qu'elles recoupent rarement. Le remplissage des fissures est constitué de quartz, épidote, calcite et leucoxène (planche 11). Dans les pillows de plus grand diamètre, une fissuration plus ou moins concentrique se combine à la fissuration précédente (fig. 19). Dans les pillows à pédoncule simple, les fentes de retrait, disposées en éventail, convergent de la croûte supérieure vers la base du pédoncule (planche 6).

Les pillows creux et les vésicules. Dans la moitié supérieure des pillows se trouvent fréquemment des cavités occupées sans doute originellement par des gaz et remplies ultérieurement par du quartz, de l'épidote (pistacite), de la chlorite, de la calcite blanche ou grisâtre et parfois du feldspath (albite) largement saussuritisé et séricitisé). Ces cavités sont plus fréquentes dans les gros pillows que dans les petits (fig. 19 et planche $7 \mathrm{a}$ et $\mathrm{b})$.

Dans certains pillows de grand diamètre, on distingue une succession de cavités allongées, parallèles entre elles et remplies secondairement par du quartz, avec un peu de calcite (fig. 20). L'aplatissement et l'allongement tectoniques de tels pillows provoquent un étirement de ces «lits » quartzitiques (p. 62).

Des vésicules sphériques blanchâtres, de 1 à $5 \mathrm{~mm}$ de diamètre, sont localisées souvent au centre ou dans la moitié supérieure des pillows (planche 6, planche $8 \mathrm{~b}$ et planche $11 \mathrm{e}$ ). Plus rarement, elles sont distribuées dans toute la lave (lave vésiculée). Nous pensons que ces vésicules correspondent à des bulles de gaz se formant dans la masse magmatique du pillow. Elles sont remplies secondairement par de l'épidote souvent zonée, de la calcite avec un peu de quartz et d'amphibole chloritisée. Tous ces minéraux sont chargés de pigment brun sale qui pourrait être du leucoxène. 


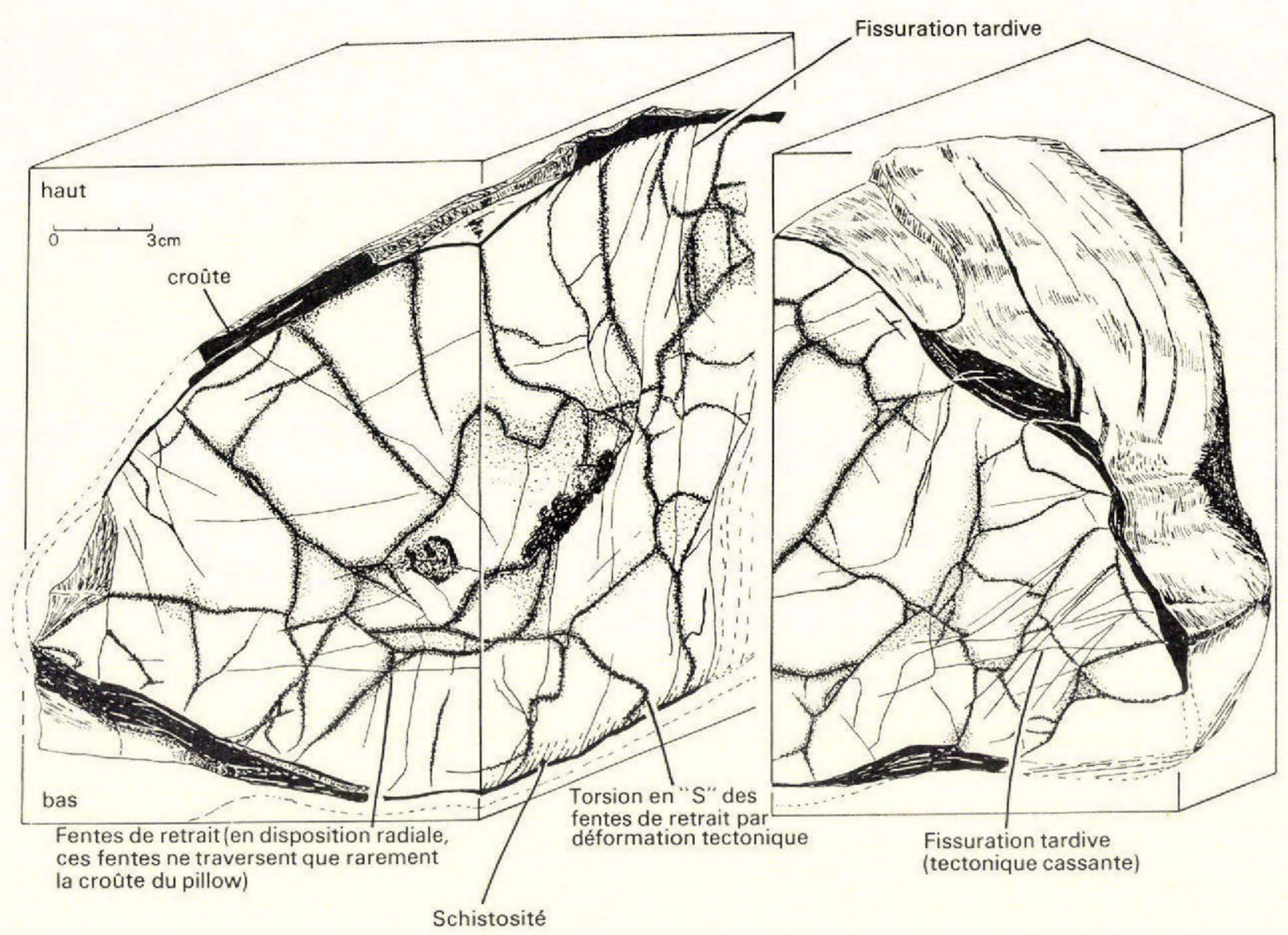

Fig. 18. Structure interne et déformations d'un pillow-lava, Pinguligssuánguaq, Arsuk $\varnothing$. GGU 32666. 


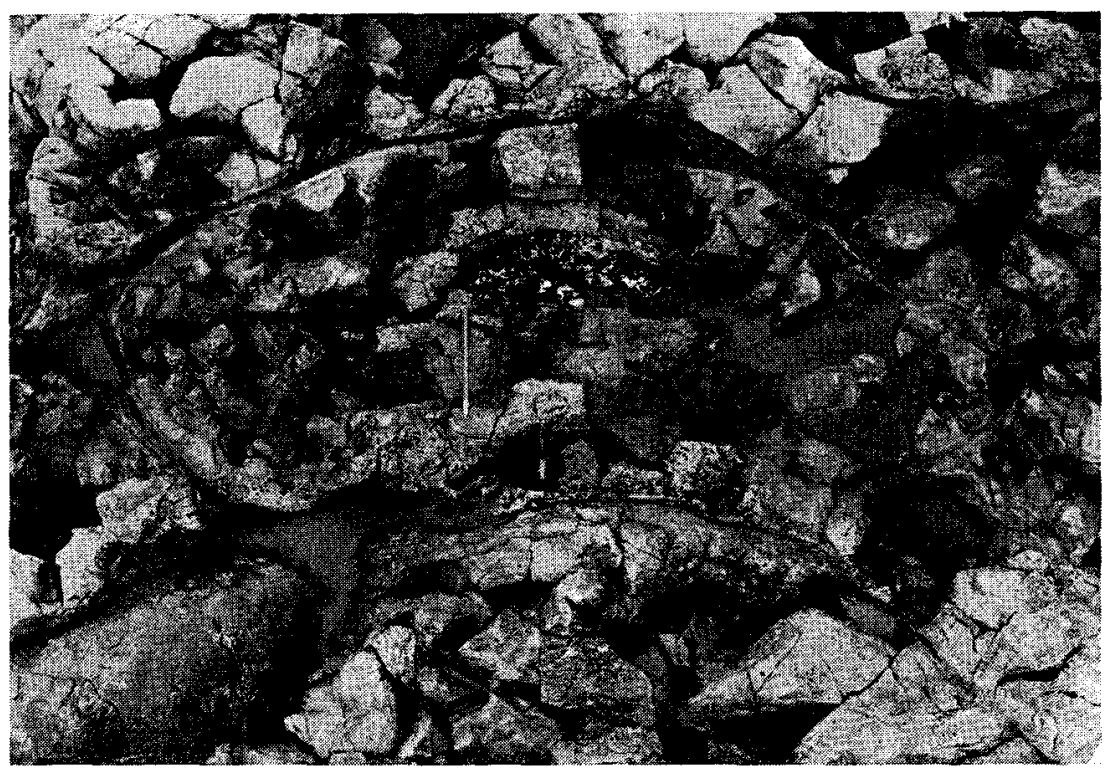

Fig. 19. Fentes de retrait radiales et concentriques dans un pillow. Unité volcanique inférieure. Pinguligssuánguaq. La base du pillow est indiquée par la pointe du crayon. Cavité remplie de quartz et carbonate dans la partie supérieure du pillow. Remplissage secondaire de carbonate dans les fissures et interstices des pillows.

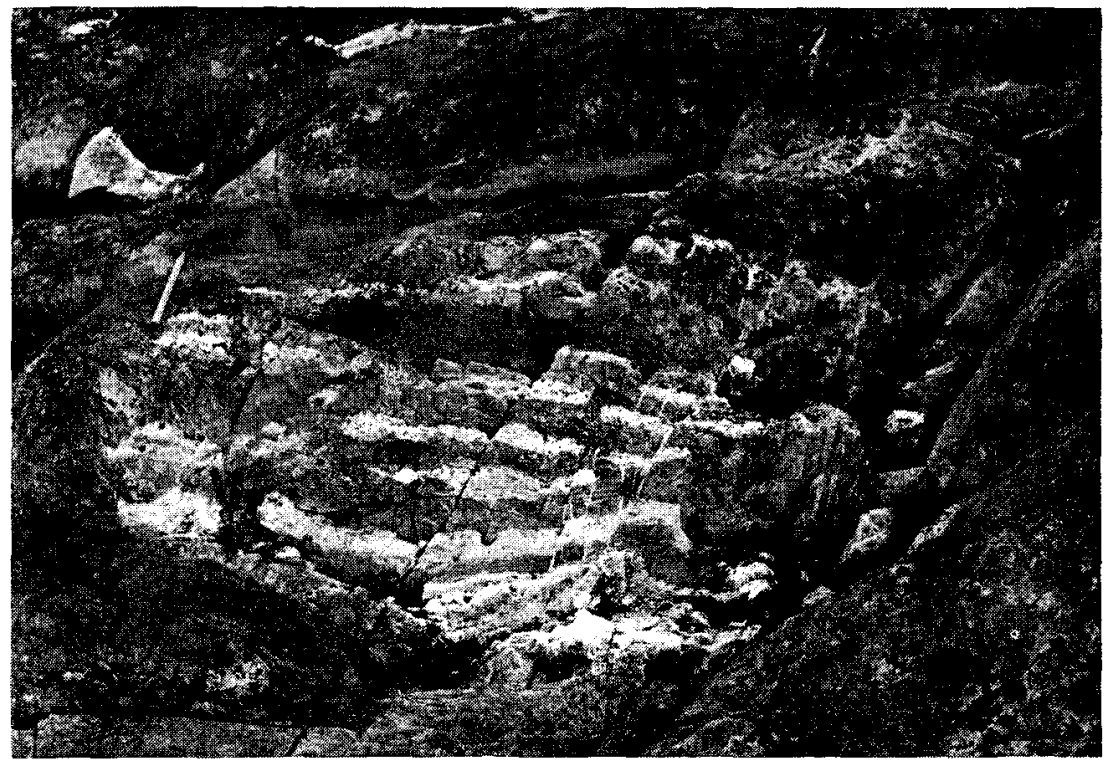

Fig. 20. Cavités parallèles et allongées remplies de quartz dans la partie supérieure d'un pillow. Région Sud-Ouest d'Arsuk $\varnothing$, au Nord de Qeqertat. 


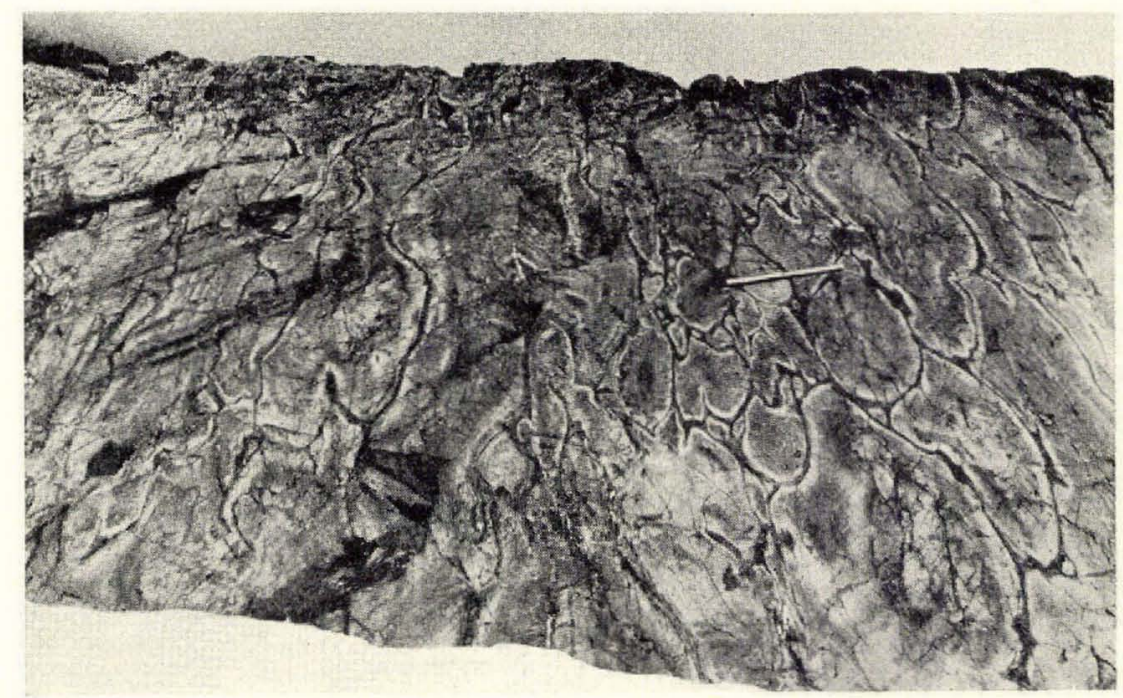

Fig. 21. Entassement de pillow-lavas de type amiboïde. Unité volcanique moyenne. Rive orientale du lac $490 \mathrm{~m}$, Arsuk $\varnothing$. Ces pillows sont bordés d'un liséré clair riche en épidote.

Les pillow-lavas amiboïdes. La forme générale de ces pillows correspond à celle de gros sacs présentant des renflements et des rétrécissements dans toute leur masse, ainsi que des invaginations et des pédoncules multiples, latéraux ou basaux, rappelant la forme des amibes avec leurs pseudopodes (fig. 21). Ce type morphologique de pillow n'a été observé que dans l'unité moyenne du Groupe volcanique d'Arsuk.

L'aplatissement et l'allongement, souvent très prononcés, des pillows amiboïdes sont dus en grande partie à des déformations tectoniques car ces pillows sont affectés d'une schistosité bien marquée.

\section{Couleur des pillows dans les différentes unités lithologiques}

Les pillows sombres. La roche constituant les pillows de l'unité moyenne du Groupe volcanique d'Arsuk est généralement de couleur assez sombre, verdâtre à noire, en raison de l'abondance de l'ilménite leucoxénisée (5-10\%, tableau 7). Dans la bordure des pillows, cette teinte s'éclaircit et devient gris clair ou gris-jaune suivant le pourcentage d'épidote qu'elle renferme (fig. 21). L'altération météorologique donne à ces roches une patine brunâtre ou brun-rougeâtre.

Les pillows clairs. Dans les unités inférieure et supérieure du groupe volcanique, les pillows ont une teinte gris-vert clair à la cassure fraîche. Par altération météorologique, la roche acquiert une patine vert-jaune, un peu rosée. 
Tableau 7. Composition minéralogique des pillows.

\begin{tabular}{|c|c|c|c|}
\hline \multirow[b]{2}{*}{$\mathrm{n}^{\circ}$ plaques GGU } & \multirow{2}{*}{$\frac{\text { pillow clair }}{32739}$} & \multicolumn{2}{|c|}{ pillows sombres } \\
\hline & & $\begin{array}{c}32772 \\
\%\end{array}$ & $\begin{array}{c}54826 \\
\%\end{array}$ \\
\hline Amphibole............. & & 10 & $10-15$ \\
\hline Chlorite ............. & $70-80$ & 50 & \\
\hline Epidote............... & & 15 & $60-75$ \\
\hline Feldspath............ & $15-20$ & $15-20$ & 15 \\
\hline Ilménite et leucoxène. . . . . . & $2-3$ & $5-7$ & $7-10$ \\
\hline $\begin{array}{l}\text { Calcite . . . . . . . . . . . . . } \\
\text { Les pourcentages sont estimés }\end{array}$ & $4-5$ & 1 & 1 \\
\hline
\end{tabular}

Lors des levés géologiques des différentes unités du Groupe volcanique d'Arsuk, nous avons utilisé les critères suivants:

pillows sombres, de type ami- Unité moyenne

boïde, parfois ellipsoïdal très

aplati

Pillows clairs, de type

ellipsoïdal

Unités de transition,

inférieure et supérieure

Dans la partie occidentale d'Arsuk $\varnothing$ et à Manîtsoq on observe à la base de l'unité supérieure quelques coulées de pillows verdâtres ou noirs, intercalées dans les coulées plus claires.

\section{Pétrographie des pillows}

Composition pétrographique des pillows clairs.

L'examen microscopique de roches provenant de pillows clairs, des unités inférieure et supérieure, révèle les caractères suivants.

Le plagioclase, en microlites de $0,25 \mathrm{~mm}$ de long sur $0,03 \mathrm{~mm}$ de large, constitue la trame de la roche. Les plus gros, légèrement zonés, ont une composition voisine de l'oligoclase acide. Ces feldspaths sont très saussuritisés et on observe parfois la présence de néoformation d'albite contenant des inclusion d'amphiboles.

L'amphibole fibreuse, incolore ou légèrement verte, partiellement chloritisée, est abondante.

L'épidote (clinozoïsite et pistacite) est abondante.

Les minéraux secondaires sont la calcite (en faible quantité), l'ilménite plus ou moins leucoxénisée sous forme de minuscules granules, le quartz (rare) et la pyrite secondaire idiomorphe.

Les pourcentages des différents constituants minéralogiques de ces roches sont donnés dans le tableau 7 . Ces pourcentages sont approximatifs, car le faible diamètre des minéraux et le développement abondant d'amphibole fibreuse n'ont pas permis de faire des mesures précises. 
La plupart des pillows clairs examinés contiennent de nombreuses pseudomorphoses de phénocristaux de $0,5 \mathrm{~mm}$ de diamètre; ces pseudomorphoses sont constituées d'actinote qui est finalement chloritisée (pennine) avec formation de sphène, calcite et parfois de quartz. Les formes cristallographiques mal conservées de ces pseudomorphoses ne permettent pas de déterminer leur nature originelle. Elles pourraient correspondre à des olivines, des pyroxènes, ou peut-être même à des amphiboles (planche 10b).

Variation de texture à l'intérieur d'un pillow clair. La texture de la roche ne varie pas considérablement à l'intérieur des pillows examinés. On note toutefois une légère diminution de la longueur des microlites de plagioclase en se rapprochant de la croûte du pillow. L'examen macroscopique de sections polies et laquées permet de déceler avec plus de finesse quelques variations concentriques. Un pillow, tel que celui que nous avons représenté sur la planche 10 (voir également planche $7 \mathrm{a}$ ), montre les variations suivantes depuis la croûte jusqu'au centre:

Juste en dessous de la croûte verdâtre à noire se trouve une zone vert foncé, de 3 à $5 \mathrm{~mm}$ d'épaisseur, s'éclaircissant rapidement en direction du centre du pillow. La teinte devient alors verte sur 5 à $8 \mathrm{~cm}$ d'épaisseur. La partie centrale du pillow est à nouveau légèrement plus foncée.

La finesse du grain de la roche est à peu près identique dans tout le pillow; dans la partie centrale, juste au-dessous de la cavité remplie secondairement par du quartz et de la calcite, se trouvent de nombreuses petites taches claires, de 1 à $2 \mathrm{~mm}$ de diamètre, qui sont des vésicules.

D'un pillow à l'autre, la teinte moyenne de la roche en section polie et laquée varie entre le vert, gris-vert, verdâtre et gris-noir. Au microscope, ces différences de teintes ne sont pratiquement pas décelables.

Variation de texture en bordure d'un pillow clair. En s'approchant de la croûte du pillow, la texture se modifie totalement. Cette variation est représentée sur les planches 10 et 11 .

A 2-3 mm de la croûte proprement dite on passe de la texture feutrée ou en "houppes", toujours très voilée par les fibres d'amphiboles, à la texture sphérolitique: de très petites "pustules" sombres, sphériques ou ellipsoïdales, dont le diamètre ne dépasse pas $0,2 \mathrm{~mm}$, s'individualisent peu à peu. On reconnait parfois dans la partie centrale de ces "pustules " quelques microlites de feldspath très saussuritisés (planche $11 \mathrm{~b}$ ), mais plus généralement elles sont constituées uniquement d'amphiboles fibroradiées. Ces amphiboles sont chargées de pigment brun sale dans le centre du sphérolite qui est encore entouré par une ou deux "coquilles » composées 
d'agrégats semi-opaques, probablement du leucoxène (planche 10c). La matrice, entre les sphérolites, est composée d'amphibole incolore, d'épidote, de calcite et de chlorite.

A 1 ou $2 \mathrm{~mm}$ sous la croûte du pillow, la chlorite presque isotrope et l'épidote deviennent les constituants principaux de la matrice. L'amphibole n'est présente alors que dans les sphérolites.

On passe ensuite à la croûte proprement dite du pillow dont l'épaisseur varie entre $3 \mathrm{~mm}$ et $1 \mathrm{~cm}$. Elle est composée de chlorite (dont une pennine) et de matière cryptocristalline, plus ou moins chargée de pigment opaque, dans laquelle sont dispersés des sphérolites jaunâtres ou brunâtres, amphiboliques et chloriteux, entourés de leur enveloppe d'agrégats brun-noir de leucoxène. On note encore dans cette zone un beau développement d'épidote idiomorphe (pistacite-clinozoüsite) disposée d'une manière sphérolitique et dont la formation est certainement postérieure aux premiers stades de transformation de la croute (chloritisation planche $10 \mathrm{a}$ et d). Ce type de minéraux sphérolitiques est tout à fait identique aux "radial epidote glomeroblasts " décrits par Misch (1965) dans les schistes actinolitiques ou à crossite de la série des schistes verts métabasaltiques de Shuksan (Washington). Selon cet auteur, ils se seraient développés dans des conditions syncinématiques, lors de la formation de la schistosité. Nos sphérolites sont, par contre, antérieurs à la schistosité qui les déforme.

Transformation de la croûte des pillows clairs. La matière cryptocristalline semi-opaque qui constitue la croûte renferme des sphérolites tout à fait isolés les uns des autres. Cette matière se transforme en chlorite vert pâle, presque isotrope, qui constitue des zones plus claires, bordées d'un liséré chargé de pigments brun-noir (leucoxénique) correspondant à un phénomène de ségrégation. On peut parler de la formation d'un "front " de fer (ilménite?) lors de la transformation en chlorite de la matière cryptocristalline semi-opaque (planche $10 \mathrm{a}$ et $\mathrm{d}$ ). Cette matière cryptocristalline provient peut-être de la transformation d'un verre. Dans quelques cas elle est de couleur jaunâtre ou jaune miel en lumière polarisée et on y reconnaît de minuscules cristaux biréfringents, jaunes, formant un fin feutrage. D'autres cristaux plus gros s'identifient à la clinozoīsite.

Le processus de transformation métamorphique de la matière cryptocristalline semi-opaque procède à partir des fissures perpendiculaires ou parallèles à la croûte du pillow et qui peuvent être liées dans certains cas à des fentes de retrait. Cette transformation, accélérée par les déformations tectoniques, atteint toute la masse de la croûte qui passe en chlorite, épidote, leucoxène, quartz, calcite et trémolite.

Paragenèse. La présence dans la croûte de pseudomorphoses de phénocristaux transformés en chlorite et actinote montre que la para- 
genèse de la lave a commencé par la formation de ces phénocristaux avant sa consolidation sous forme de pillow. Il n'a pas été possible de définir si ces phénocristaux correspondaient à d'anciennes olivines, amphiboles ou éventuellement des pyroxènes. Une paragenèse débutant par la formation d'augite est fréquemment signalée dans la littérature, notamment par Salimi (1965) qui l'observe dans les pillows spilitiques des Préalpes romandes.

Composition minéralogique des pillows sombres.

L'examen microscopique de roches schisteuses provenant de pillows sombres amiboïdes révèle les caractères suivants:

Les microlites de feldspath ont une composition qui varie, suivant les roches examinées, entre l'albite et l'oligoclase. Ces feldspaths sont réduits en grains. Ils sont parfois recristallisés avec inclusions de chlorite.

L'amphibole, légèrement pléochroïque $(\alpha=$ jaune ou incolore; $\beta=$ vert; $\gamma=$ vert-bleuté) est cataclasée, fragmentée et très chloritisée.

La chlorite verte (pennine) se dispose parallèlement à la schistosité et enveloppe des "chapelets " de granules leucoxéniques.

La pyrite est fréquente. On observe parfois un peu de biotite brune.

Les pourcentages des différents constituants minéralogiques de ces roches sont donnés dans le tableau 7.

\section{Le matériel interstitiel dans les coulées de pillows}

Le matériel interstitiel est représenté par plusieurs types de roches parmi lesquelles on distingue:

Un matériel schisteux, chloritofeldspathique ou chloritoquartzeux à épidote et ilménite, provenant probablement de la transformation de verres.

Des tufs. Ils annoncent souvent un arrêt des coulées de lave en pillows auxquelles succède alors un dépôt de tufs et de lapillis.

Des petits pillows emballés dans des tufs et des lapillis. Un matériel quartzeux auquel s'ajoute parfois un matériel carbonaté.

Le matériel schisteux chloritoquartzeux à épidote. Dans les interstices des pillows sombres de l'îlot Manitsoq, le matériel schisteux, chloritoquartzeux, à biotite verte et ilménite, renferme des "lits》 d'épaisseur millimétrique, très plissotés et boudinés, constitués exclusivement $d$ ' épidote.

Le matériel quartzeux. De couleur blanche ou noire, il consiste en un assemblage de grains de quartz équidimensionnels, avec un peu de carbonate et de magnétite en minuscules cristaux idiomorphes disposés en traînées ou rassemblés en taches. Parfois il s'y ajoute un peu d'épidote et de trémolite. Ce matériel semble correspondre à un chert recristallisé. 
Cette origine est généralement admise dans la littérature (Реттылоn, 1957, Carlisle, 1963 et Sampson, 1923). Comme le chert recristallisé est impossible à distinguer d'un quartzite métamorphique (Dunbar \& Rodgers, 1957), l'origine purement détritique de ce matériel n'est pas exclue à priori. Cependant, l'examen de 15 plaques minces ne nous a pas permis de reconnaître un seul minéral détritique tel que le zircon, la tourmaline, le rutile, etc., que l'on pourrait s'attendre à trouver dans un sédiment d'origine terrigène.

L'examen de la planche 5 permet de voir que la boue siliceuse interstitielle, constituée de matériel quartzeux (en blanc sur la photographie) auquel se mêle également du tuf (en gris clair), a été déformée peu après son dépôt, probablement lors du tassement consécutif à l'empilement des pillows dans la coulée. Cette déformation syngénétique semble exclure la possibilité d'un remplissage tardif de quartz par solution hydrothermale. On peut donc se demander si le matériel quartzeux est d'origine détritique (sable) ou bien chimique (chert, p. 79). La recristallisation du quartz ne nous permet pas de préciser son origine.

Le matériel carbonaté. Les interstices entre les pillows sont remplis fréquemment par un matériel carbonaté blanc ou grisâtre, contenant de la magnétite, ainsi qu'un minéral noir opaque (graphite?). Le carbonate est partiellement remplacé par de la trémolite vert pâle fibreuse. Il est dif ficile de préciser si ce carbonate correspond à un remplissage primaire (précipité chimique lié au volcanisme: KANIA, 1929) ou secondaire (solution hydrothermale). La présence d'un matériel tout à fait analogue dans les cavités des pillows creux (fig. 19) indiquerait plutôt une origine hydrothermale.

\section{Déformations tectoniques des pillow-lavas}

Plusieurs stades de déformation ont été mis en évidence dans les pillows d'Arsuk $\varnothing$. Dans un premier stade, on observe de nombreuses petites fissures obliques à la bordure du pillow qui déterminent des tranches plus ou moins parallèles entre elles. Ces tranches, en glissant les unes sur les autres, provoquent l'aplatissement et l'allongement relatif du pillow (planches 7 a et 8 a). Les fissures ouvertes sont cicatrisées par de l'amphibole incolore, de la calcite, du quartz, de la chlorite et de l'épidote. La texture de la roche à l'intérieur des tranches est à peine modifiée. Ce débitage du pillow en "tranches " pourrait correspondre à une schistosité de fracture. Lors de ce processus de déformation, les fentes de retrait sont parfois utilisées comme surface de mouvement. Celles qui se trouvent "en zones " avec la schistosité sont tordues en forme de $S$, indiquant de ce fait le sens relatif des déplacements qui se sont produits de part et d'autre du pillow (fig. 18). 


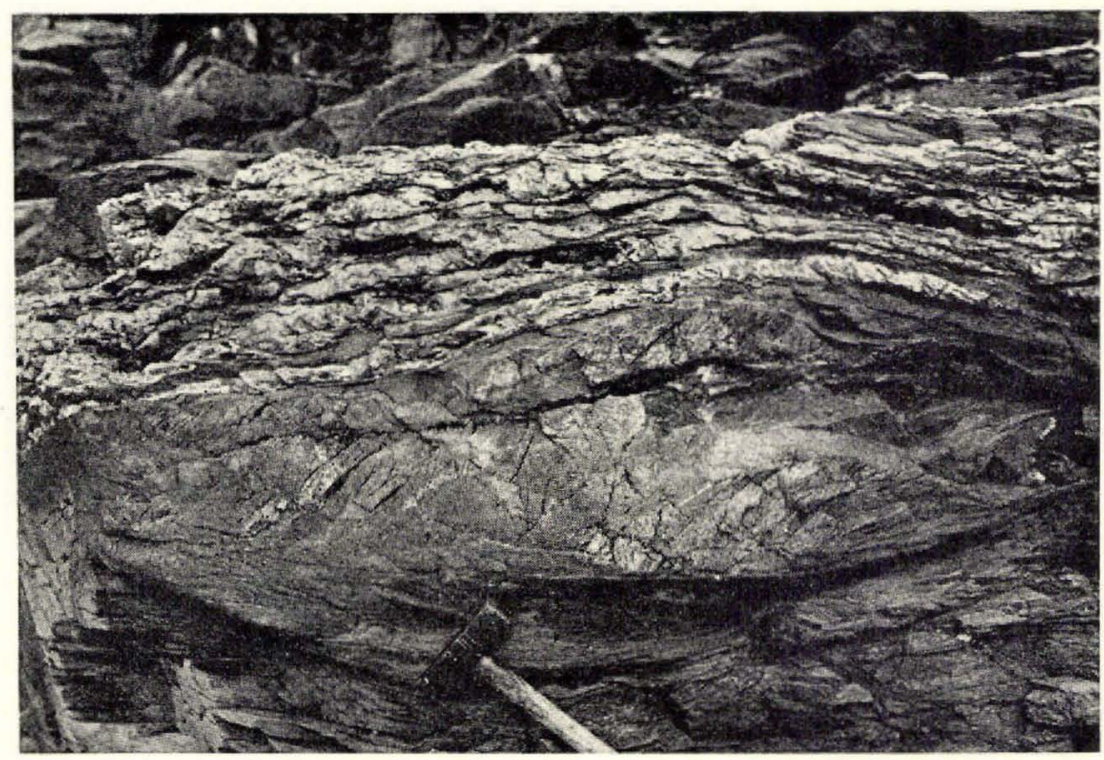

Fig. 22. Déformation d'un pillow-lava contenant, dans la partie supérieure, plusieurs cavités allongées remplies de quartz. Arsuk $\varnothing$, côte Sud-Ouest, à l'Est d'Arferutdluk. Dans la zone externe du pillow qui est très schisteuse, les "lits " quartzeux sont étirés. Dans la partie centrale du pillow, en observe des fractures de tension.

Un autre stade de déformation correspond à l'apparition de la schistosité de flux. Celle-ci n'affecte d'abord que la croûte du pillow qui est la plus fragile. La chlorite se dispose parallèlement aux feuillets schisteux et renferme des chapelets de leucoxène. Les petits sphérolites d'amphiboles sont étirés et prennent des formes ellipsoïdales très allongées jusqu'à n'être plus reconnaissables. Les épidotes sont cassées et emballées par les minéraux précédents (planche $11 \mathrm{~h}$ ). Les zones plus riches en épidote sont déformées d'une manière cassante avec apparition de fissures pennées. Les fissures ouvertes sont alors remplies de minéraux tels que chlorite, épidote, albite, carbonate et parfois pyrite.

Un stade de déformation plus avancé correspond à l'acquisition, par presque toute la roche constituant le pillow, d'une schistosité beaucoup plus nette que la précédente. A ce stade, les cavités allongées, remplies de quartz, qui se trouvent dans la moitié supérieure des pillows, sont généralement très étirées (fig. 22).

Dans le stade de déformation suivant, toute la roche du pillow devient schisteuse. Au microscope, on observe une cataclase des feldspaths et des amphiboles et une abondante formation de chlorite dont les feuillets se disposent parallèlement aux plans de schistosité. Ces déformations intimes de la roche sont accompagnées par un allongement et un aplatissement des pillows. Finalement, les structures macroscopiques des pillows dis- 


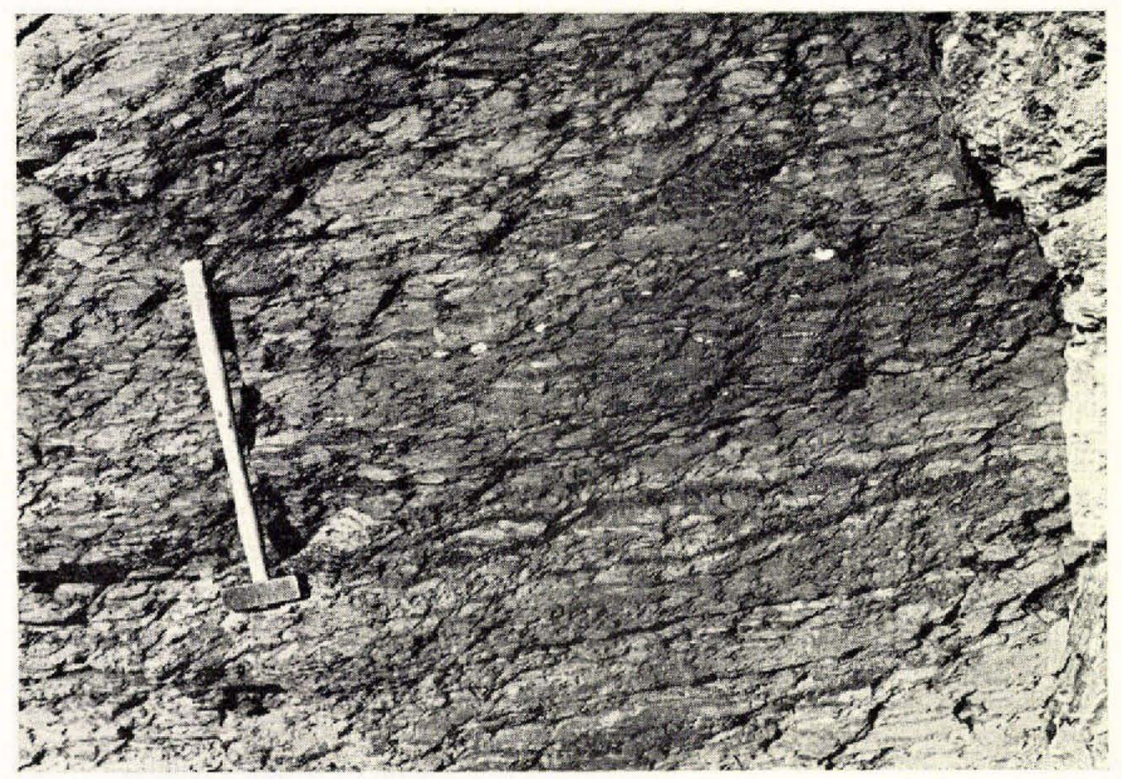

Fig. 23. Pillow-lavas de type amiboïde, étirés et aplatis. Unité moyenne. Rive droite du lac $490 \mathrm{~m}$, Arsuk $\varnothing$. Les limites des pillows sont encore reconnaissables sous forme de bandes sombres parallèles à la schistosité. Cette schistosité est elle-même recoupée par des plans de cisaillement.

paraissent presque totalement. Il ne subsiste alors que des reliques de croûte qui s'allongent en bandes parallèles (fig. 23). Ce type de déformation affecte particulièrement les pillows sombres de l'unité moyenne.

\section{Transformations métamorphiques des pillow-lavas}

A part quelques microlites d'albite (disposés suivant la texture en "houppes") et des reliques d'ilménite, tous les minéraux constituant les pillows examinés sont secondaires. L'épidote, la calcite, une partie de l'albite, l'amphibole, la chlorite et le leucoxène sont des produits de transformation métamorphique. Ils proviennent de la saussuritisation de plagioclases, de la transformation des minéraux ferromagnésiens primaires, de la chloritisation des amphiboles et de la leucoxénisation de l'ilménite. Des transformations analogues ont été observées par Vuagnat (1948) dans certaines diabases épidotiques alpines. La matière cryptocristalline semi-opaque de la croûte des pillows provient peut-être de la transformation d'un verre.

Les textures typiques des pillows peu transformés, comme ceux des Préalpes et de Basse Engadine (Vuagnat, 1948 et 1951; Salimi, 1965) n'ont pas été reconnues ici. On peut même se demander si ces textures ont existé dans les pillows d'Arsuk avant leur transformation métamorphique. 
Quelle était la nature des ferromagnésiens primaires? On peut la supposer:

pyroxène (augite) et olivine:

par analogie avec les pillows peu transformés observés dans d'autres régions

amphibole (hornblende brune):

par analogie avec les laves massives associées aux coulées de pillows d'Arsuk $\varnothing$ et les filons basiques kétilidiens riches en amphibole brune (p. 76).

ilménite:

reliques préservées.

La composition initiale du feldspath est difficile à préciser, car celuici est en général très altéré. Dans certains pillows clairs, le feldspath est représenté par des microlites d'albite disposés suivant la texture primaire en "houppes " (planche $11 \mathrm{~d}$ ). Cette albite peut correspondre à la composition initiale du plagioclase. Mais la possibilité d'un remplacement, lors des transformations métamorphiques, du calcium d'un plagioclase (qui serait primitivement plus calcique) par du sodium sans altération de la disposition en "houppes ", peut être aussi envisagée. Dans d'autres pillows clairs, dans lesquels les microlites de feldspath sont très saussuritisés et où la texture en "houppes » est à peine reconnaissable, on observe parfois des néoformations d'albite microgrenue. La composition initiale du plagioclase de tels pillows était sans doute plus calcique que cette albite qui est un produit de métamorphisme. Dans certains échantillons, la présence de petits phénocristaux de plagioclase légèrement zonés (albite à oligoclase acide) confirmerait cette hypothèse. Dans les pillows sombres nous avons mesuré des compositions allant de l'albite à l'oligoclase. Le plagioclase qui a cristallisé dans les cavités des pillows creux (p. 53) devait avoir aussi une composition plus calcique que l'albite saussuritisée qui s'y trouve actuellement.

Ces observations montrent qu'il a dû y avoir dans la plupart des pillows un plagioclase calcique qui, par suite de la "rétromorphose" subie par la roche, fut transformé en albite. Cette composition calcique correspond-elle à la composition originelle du feldspath magmatique ou bien caractérise-t-elle seulement une étape de l'histoire de ce minéral (étape correspondant à la cristallisation de feldspath calcique dans les cavités des pillows)? L'examen microscopique à lui seul ne permet pas de résoudre ce problème.

\section{Mise en place des pillow-lavas du Groupe volcanique d'Arsuk}

La formation des structures en pillows par refroidissement brutal de la lave venant en contact avec de l'eau est généralement admise dans 
la littérature. La comparaison des pillows d'Arsuk $\emptyset$ avec ceux constituant des coulées subaquatiques récentes nous permet de considérer cette hypothèse comme la plus vraisemblable (Anderson, 1910; Moore \& ReEd, 1963).

Les pillows d'Arsuk $\emptyset$ sont fréquemment reliés entre eux. Ces connections ne sont pas visibles dans tous les cas, notamment lorsqu'on examine les diaclases ou les surfaces d'érosion recoupant les entassements de pillows dans une seule direction. Le fort relief d'Arsuk $\varnothing$ et son érosion intense, accélérée par la présence d'un réseau de diaclases de directions variées, nous a permis d'observer une multitude de petits «blocs diagrammes " naturels. Nous prendrons un exemple dans la moitié occidentale d'Arsuk $\varnothing$ où les structures sont assez simples puisque les couches ont une direction sensiblement $\mathrm{E}-\mathrm{W}$, plongeant toutes au Sud. Les connections entre les pillows sont peu fréquentes lorsqu'on examine les entassements de pillows suivant les plans de diaclases E-W, plus ou moins perpendiculaires aux couches (ces plans sont les mieux marqués dans la topographie). Par contre, les connections semblent plus nombreuses lorsqu'on observe les sections de pillows suivant des plans de diaclases $\mathrm{N}-\mathrm{S}$.

Des connections entre pillows ont également été observées par M. E. Wilson (1962) dans les séries volcaniques précambriennes du Comté d'Hasting en Ontario. Nous en avons vu également de nombreux exemples au Québec, dans les coulées précambriennes de la région de Rouyn-Beauchastel ainsi que dans les coulées paléozoïques de Frontenac près du lac Mégantic dans la châne des Appalaches.

La présence de connections entre différents pillows permet d'établir quelques hypothèses relatives à la formation de ce type de structure. Il nous semble vraisemblable que les pillows aient été, dans une certaine mesure, connectés entre eux lors de leur formation, la lave passant de l'un à l'autre et se propageant ainsi de plus en plus loin de la source d' émission. Cette mise en place des pillows correspond à la théorie du "bourgeonnement bulbeux» (bulbous budding) de Lewis (1914) reprise avec quelques variantes par d'autres auteurs (voir également VuAGNat \& Pustaszeri, 1965). Dans certains cas, les pillows ainsi formés se sont détachés de leur ombilic, puis ont roulé "sur les flancs ou le front de la coulée en s'empilant les uns sur les autres, les plus récents, done les plus chauds, s'adaptant aux précédents déjà refroidis 》 (Ritrmann, 1963, p. 122).

Il est possible aussi que les coulées de lave massive aient joué le rôle de "canaux nourrisseurs" dans ce cheminement de la lave à des distances plus grandes encore (p. 71).

La présence de connections entre différents pillows peut également s'expliquer par une exagération de la structure cordée observée dans les 
coulées subaériennes. La surface des coulées sous-marines récentes de la région des îles Hawaï, photographiées à des profondeurs allant jusqu'à $5000 \mathrm{~m}$ environ, présentent de telles structures (Moore \& REED, 1963).

Bourgeonnement bulbeux, boules de lave roulant sur les pentes de la coulée, exagération de la structure cordée ... L'application d'une seule cause ne peut traduire les différentes possibilités de mise en place des coulées sous-marines.

La structure morphologique particulière des pillows amiboïdes et la composition de leur croûte si riche en épidote pourraient être liées aux conditions physico-chimiques du milieu dans lequel ces pillows se sont formés. Un refroidissement moins brutal de la lave pourrait expliquer les formes très contournées de ces pillows.

\section{Les coulées de pillows bréchiques ou isolés}

Des coulées de pillows bréchiques de 1 à $5 \mathrm{~m}$ d'épaisseur surmontent localement les coulées de pillow-lavas, notamment dans l'unité inférieure du Groupe volcanique d'Arsuk où elles sont les plus typiques. Elles peuvent passer, vers le haut ou latéralement, à des lapillis ou à des tufs, ou encore à des brèches volcaniques détritiques. Toutes ces roches, qui présentent des variations rapides de faciès, ont été représentées sur la planche 12 par un symbole unique (petits triangles).

\section{Caractères morphologiques et pétrographiques des coulées}

Les coulées de brèches sont constituées de fragments anguleux de pillows noyés dans une matrice assez fine qui ressemble à un tuf. La grandeur des fragments bréchiques varie entre 1 et $10 \mathrm{~cm}$. Les plus gros atteignent 20 à $30 \mathrm{~cm}$ de diamètre. Il n'y a aucun triage. A ces fragments anguleux s'ajoutent localement des petits pillows de formes amiboïdes (fig. 24). Ces coulées de brèches sont identiques à celles décrites minutieusement par Carlisle (1963) dans les coulées de pillows triasiques de Colombie britanique. L'examen de sections polies et laquées permet de préciser les caractères morphologiques des fragments et de connaitre leur relation avec la matrice qui les renferme (planche 9).

Les fragments. Les fragments de gros diamètre sont constitués par des pillows presque complets, de forme variable, subsphérique, ellipsoidale aplatie ou amiboïde. La croûte qui entoure ces pillows est parfois coupée net, comme si elle avait été arrachée au reste du pillow (planche 9).

Les petits fragments sont constitués d'une part de petits pillows de 1 à $5 \mathrm{~cm}$ de diamètre et d'autre part de brèches plus ou moins anguleuses de même dimension. Les brèches anguleuses sont constituées exclusivement de morceaux de pillow. Si elles proviennent de la bordure d'un gros 


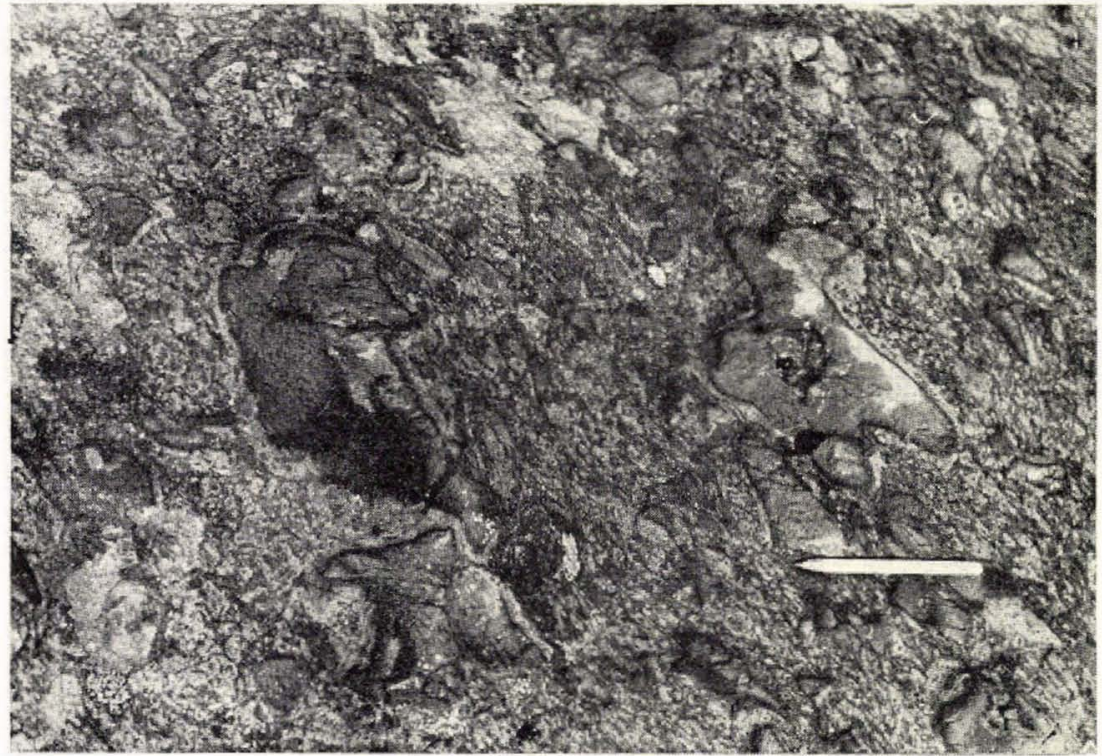

Fig. 24. Coulée de pillows bréchiques et isolés. Blăbærelv, Arsuk Ø, côte Nord. Forme amiboïde caractéristique des pillows isolés, bordés entièrement de croûte de refroidissement.

pillow, elles sont partiellement entourées de croûte, tandis que si elles correspondent à la partie interne d'un pillow, elles n'en ont pas. Les fragments anguleux sans croûte ont une bordure plus sombre qui indique qu'il y a eu une réaction entre ces fragments et le milieu dans lequel ils sont tombés. La forme courbée, sans cassure, des fragments allongés, ajoutée à l'observation précédente, montre que les morceaux n'étaient pas complètement refroidis au moment de leur dépôt et que certains pouvaient même encore être déformés plastiquement.

Les fragments provenant de la division d'un ou de plusieurs pillows ont été complètement mélangés car il est impossible de reconstruire un même pillow à partir de fragments gisant côte à côte dans une même brèche.

Les petits pillows isolés de forme amiboïdes ont des formes très variées et souvent on observe des liaisons de l'un à l'autre par un long et étroit pédoncule.

Les structures, textures et compositions pétrographiques de la croûte et de l'intérieur de ces pillows, petits ou gros, sont tout à fait identiques à celles que nous avons décrites précédemment dans les coulées usuelles de pillows jointifs: fissuration radiale ou concentrique, partie centrale parfois creuse, lave vésiculée, structure concentrique caractérisée par la variation de teinte du pillow qui reflète une différence de composition et de texture de la roche, notamment en bordure. 
Le matériel interstitiel. Le matériel interstitiel entre les fragments est composé principalement de chlorite et d'épidote avec un peu de trémolite et du feldspath très saussuritisé. On y trouve en outre des "granules " et "échardes " de matière plus ou moins opaque qui doit correspondre à des verres transformés. Les "granules》 ou "échardes" ont des dimensions allant de quelques millimètres à $1 \mathrm{~cm}$. La partie centrale de ces "granules ", constituée de chlorite presque isotrope parfois chargée de pigment brun sale, est auréolée de matériel feldspathique (albite) avec un peu de quartz et d'épidote (clinozoïsite). Un ou plusieurs lisérés de substance opaque brunâtre, provenant sans doute de l'exudation de minerai de fer contenu primitivement dans le verre, entourent encore ces structures. Autour des "granules " et des "échardes", on distingue parfois une texture fluidale due à l'arrangement d'agrégats opaques. Cette fluidalité pourrait être primaire ou formée secondairement par ségrégation lors de la transformation du matériel initial. Dans certains cas, les "granules" sont fissurés ou tronçonnés en minuscules fragments.

\section{Mise en place des "coulées" de pillows bréchiques et isolés}

La forme bréchique des pillows doit s'expliquer par des phénomènes d'éclatement: au fur et à mesure de leur formation, les pillows ont éclaté sur le lieu même de leur dépôt.

Le matériel interstitiel est formé de débris détachés de la croûte par action thermique lors du refroidissement et probablement aussi par action mécanique lors du mouvement de la coulée de brèche. Une partie de cette matrice s'est sans doute formée directement à partir de gouttelettes de lave refroidie très brusquement (CARLisLe, 1963). Il s'est ajouté parfois des tufs ne présentant aucune structure stratifiée et qui peuvent, sur quelques mètres, se substituer complètement aux brèches.

La formation des brèches que nous venons de décrire peut être liée à des projections volcaniques tombant dans l'eau. De tels phénomènes ont été maintes fois décrits dans la littérature (ANDERSON, 1910; LEwIS, 1914).

Les petits pillows amiboïdes à longs pseudopodes peuvent correspondre à des injections de lave dans le matériel tufacé interstitiel probablement gorgé d'eau mais non encore consolidé pour permettre l'étalement de la lave poussant des pseudopodes dans toutes les directions. Le refroidissement a dû être néanmoins assez rapide pour provoquer, en bordure de ces laves, des textures de refroidissement identiques à celles que nous avons observées dans les pillows normaux. L'absence de vésicule en "pipe » dans ce type de pillow (Woov, 1964) s'explique par leur faible diamètre permettant ainsi à la vapeur d'eau de se dégager tout autour d'eux. 


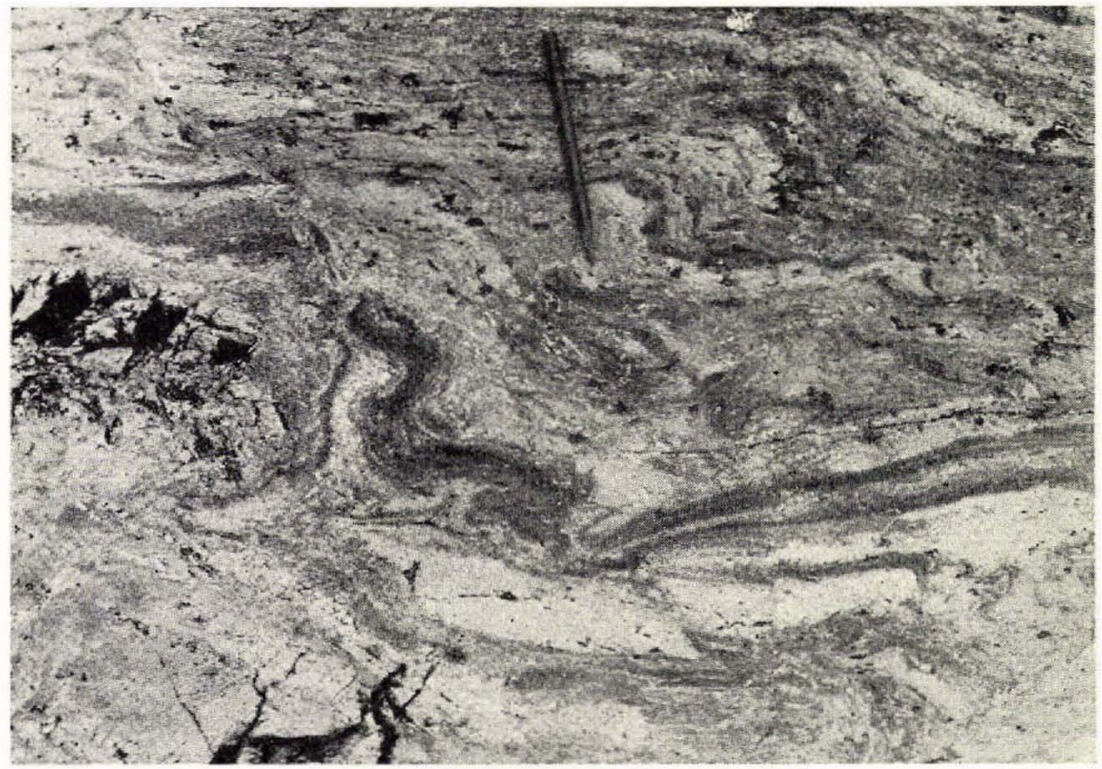

Fig. 25. Déformation syngénétique d'un tuf rubané intercalé dans les coulées de pillows. Arsuk Ø, côte Sud-Ouest, au Nord-Ouest de Qeqertasugssuk.

Les lapillis et les tufs

Des bancs rubanés de lapillis de 0,5 à $8 \mathrm{~m}$ d'épaisseur sont intercalés dans les coulées de pillow-lavas du Groupe volcanique d'Arsuk. Le rubanement est dû principalement au granoclassement et parfois à une ségrégation feldspathique tardive concordante avec la schistosité. Les fragments, de forme ellipsoïdale aplatie, ont des dimensions variant entre 3 et $30 \mathrm{~mm}$ suivant le grand axe de l'ellipsoïde. Par granoclassement et par variations latérales dans un même banc, en passe aux cendres grossières et aux cendres fines (FISHER, 1961) que nous avons rassemblées ici sous la dénomination commune de tuf (diamètre inférieur à $2 \mathrm{~mm}$ ). Les lapillis et les tufs ont été représentés sur la planche 12 par un symbole unique (trait interrompu).

\section{Les lapillis}

Les fragments. De couleur vert clair blanchâtre ou verdâtre, les fragments sont constitués de matériel feldspathique extrêmement fin, saussuritisé.

Le feldspath déterminable a une composition proche de l'oligoclase. Les autres constituants sont, par ordre d'importance, la chlorite, l'épidote et la trémolite secondaire dont les fibres parviennent à masquer complètement les textures. Le carbonate et le leucoxène sont également présents en faible quantité. 
La matrice. La constitution pétrographique de la matrice est identique à celle des fragments, mais les minéraux sont plus gros et les textures différentes.

La chlorite pléochroïque et l'amphibole incolore (trémolite?) s'allongent parallèlement à la schistosité. Le feldspath microgrenu est un oligoclase acide. L'épidote idiomorphe est zonée de la pistacite à la clinozoïsite et contient des inclusions de leucoxène.

\section{Les tufs}

Les tufs ont la même composition pétrographique que la matrice des lapillis. Ils sont souvent rubanés et montrent parfois des figures de plissement syngénétique dues probablement au poids et au mouvement des coulées de pillows qui les ont surmontés (fig. 25).

Quelques minces niveaux de phyllites noirs pyriteux accompagnent fréquemment les tufs. Ils sont constitués de matériel quartzochloriteux extrêmement fin, avec un peu de feldspath et de biotite brune.

\section{Les brèches volcaniques détritiques}

Les brèches volcaniques détritiques sont constituées de fragments de pillows, de gabbro-diorites, de phyllites noirs chloritoquartzeux ilménitiques et parfois de tufs s'infiltrant entre les fragments.

Généralement, ces brèches reposent sur les coulées de pillows. On observe toutes les transitions entre les pillows faiblement désagrégés, encore en place dans la coulée, puis les brèches anguleuses qui témoignent d'un transport faible, et enfin les brèches polygéniques à fragments mieux calibrés et un peu émoussés. Le transport n'a cependant jamais été très long et il s'agit, dans la plupart des cas examinés, d'un remaniement presque sur place.

Le matériel interstitiel est constitué de tufs remaniés ou de matériel chloritoquartzeux noir, ou encore de matériel quartzeux blanc identique à celui qui se trouve entre les pillows (p. 60).

Un banc de brèches caractéristiques se trouve au Sud-Ouest de Fladedal, près du point $225 \mathrm{~m}$. Ce banc fait suite à une coulée de pillows bréchiques située plus à l'Est. Il est probable qu'une partie du matériel provienne de cette coulée de pillows bréchiques. Mais la présence de gabbrodiorite indique aussi un apport plus lointain car, dans ce secteur, la brèche détritique n'est nulle part en contact avec des laves massives gabbro-dioritiques.

Les laves massives associées aux coulées de pillows Caractères morphologiques

Des coulées massives de composition gabbro-dioritique sont intercalées dans les coulées de pillow-lavas d'Arsuk $\varnothing$. Elles se distinguent de 
ces dernières par l'absence de structure de refroidissement, par leur allure massive et la présence de nombreuses taches vertes d'amphibole ayant jusqu'à $0,5 \mathrm{~cm}$ de diamètre (qui représente en moyenne 50 à $60 \%$ du volume de la roche), noyées dans une matrice blanchâtre de feldspath saussuritisé (constituant 40 à $50 \%$ du volume de la roche). Parfois il est possible de reconnaître à l'oeil nu une disposition ophitique des lattes de plagioclase entre lesquelles s'enchevêtrent les amphiboles.

L'épaisseur de ces coulées est très variable. Les plus minces, qui n'ont que 2 à $3 \mathrm{~m}$ d'épaisseur, sont difficiles à cartographier car le grain fin et la couleur de la roche sont tout à fait semblables à ceux des pillows. Dans les coulées plus épaisses et atteignant 300 à $400 \mathrm{~m}$, comme dans l'unité inférieure du groupe volcanique, le grain est généralement plus grossier dans la partie centrale de la coulée.

La distribution des laves massives est très irrégulière. A l'échelle de la carte géologique au 1:20000, on est frappé par leur allure ramifiée, digitée ou "en relais ». Sur le terrain, aucune discordance entre les coulées massives et les coulées de pillows n'a pu être mise en évidence, car il n'existe nulle part de contact franc. Le plus souvent, on observe entre ces deux types de coulées une zone de transition de quelques décimètres à plusieurs mètres d'épaisseur dans laquelle les roches ont un grain très fin et où il n'y a pas de structure de pillows.

Dans les coulées de laves massives les plus importantes, nous n'avons jamais observé de structure en "orgues " due à la contraction de la lave lors de son refroidissement, comme on peut le voir dans certaines laves massives associées aux coulées de pillows de Nouvelle-Zélande (BARTrum, 1930).

\section{Couleur des laves massives gabbro-dioritiques}

Les laves massives changent de teinte, comme les pillows, suivant l'unité lithostratigraphique dans laquelle elles se trouvent:

unités de transition, inférieure et supérieure

laves massives vert clair (pillows vert clair)

unité moyenne

laves massives vert très sombre (pillows vert sombre à noirs)

\section{Composition pétrographique des gabbro-diorites}

L'examen microscopique de roches gabbro-dioritiques vert clair non déformées, à grain fin ou moyen, révèle les caractères suivants.

Les lattes de plagioclase, en disposition ophitique, ayant jusqu'à $0,5 \mathrm{~mm}$ de long, sont englobées partiellement ou totalement dans de larges amphiboles poecilitiques dont le diamètre se situe entre 0,25 et $3 \mathrm{~mm}$ (planche $4 \mathrm{c}$ ). Le plagioclase est parfois zoné de l'albite à l'andésine acide; il est très saussuritisé. Quelques détermina- 
Tableau 8. Composition des plagioclases des gabbro-diorites.

\begin{tabular}{|c|c|c|c|c|c|c|c|}
\hline \multirow{2}{*}{$\begin{array}{l}\mathbf{n}^{\circ} \text { plaque } \\
\text { GGU }\end{array}$} & \multirow[t]{2}{*}{ Loi de macle } & \multirow{2}{*}{$\begin{array}{c}\text { Valeur } \\
\text { An } \% \\
\text { (1) }\end{array}$} & \multicolumn{3}{|c|}{$\begin{array}{l}\text { Valeur des } \\
\text { angles }\end{array}$} & \multirow{2}{*}{$\begin{array}{l}\text { Haute } \\
\text { temp. } \\
\text { An } \% \\
(2)\end{array}$} & \multirow{2}{*}{$\begin{array}{c}\text { Basse } \\
\text { temp. } \\
\text { An } \% \\
(2)\end{array}$} \\
\hline & & & $\alpha \alpha$ & $\begin{array}{l}\beta \beta \\
(2)\end{array}$ & $\gamma \gamma$ & & \\
\hline 38719 (a) & albite. & $30-30$ & 15 & 32 & 31 & $48-28-27$ & $45-32-33$ \\
\hline 32704 (a) & albite. & $29-27$ & 13 & 28 & 30 & $47-27-26$ & $43-30-32$ \\
\hline 32704 (a) & albite-ala. & $29-30$ & 6 & 28 & 30 & $36-25-27$ & $31-30-32$ \\
\hline 38878 (a) & albite-ala. & $27-25$ & 15 & 150 & 150 & $40-26-26$ & $37-31-31$ \\
\hline 48831 (a) & ala b.. & $30 \rightarrow 30$ & 166 & 13 & 8 & $40 \rightarrow 41$ & $36-37$ \\
\hline $32731(\mathrm{~b})$ & albite-ala. & $27-30$ & 5 & 28 & 29 & $35-26-27$ & $30-30-31$ \\
\hline $32731(\mathrm{~b})$ & albite-ala. & $30-31$ & 6 & 33 & 32 & $36-36-28$ & $31-31-30$ \\
\hline 32731 (b) & albite-ala. & $29-30$ & 6 & 30 & 30 & $35-26-27$ & $31-31-32$ \\
\hline 32778 (c) & albite-ala. . & $27-25$ & 16 & 150 & 29 & $43-27-26$ & $37-30-30$ \\
\hline
\end{tabular}

(1) Le pourcentage d'An des plagioclases est obtenu à partir de la table 2 de Reinhard, (1931).

(2) Le pourcentage d'An des plagioclases est obtenu à partir des courbes $\alpha \alpha, \beta \beta$, $\gamma \gamma$ correspondant aux angles des axes optiques $(\beta \beta)$ et des bissectrices $(\alpha \alpha$ et $\gamma \gamma)$ pour les hautes et basses températures des plagioclases (TrögER, 1959 et Sørensen, 1950).

(a) Gabbro-diorite clair de l'unité inférieure volcanique.

(b) Gabbro-diorite sombre de l'unité moyenne.

(c) Gabbro-diorite clair de l'unité inférieure.

tions faites à la platine universelle sont données dans le tableau 8. L'utilisation des abaques de KöHLER montre que ces feldspaths sont plutôt du type "basse température " (KöhleR, 1950; Tröger, 1959).

On reconnaît les reliques d'une amphibole $I$, brune, pléochroïque $(\alpha=$ jaunebrun; $\beta=$ brun ou brun-vert; $\gamma=$ brun foncé ou brun-vert foncé). Cette amphibole est transformée, partiellement ou totalement en actinote II, incolore ou pléochroïque

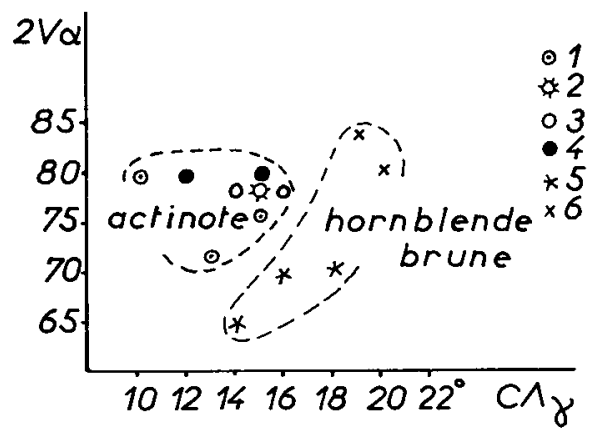

Fig. 26. Caractères optiques des amphiboles dans les gabbro-diorites, filons-couches et ultrabasites. 1: gabbro-diorite de Kînâlik (actinote). 2: Filon-couche au Sud d'Ikerasârssuk (actinote). 3: gabbro-diorite de Krabbenæs (actinote). 4 et 5: hornblendite d'Ivît Elv (hornblende et actinote). 6: péridotite à hornblende du ravin Nord-Est de Krabbenæs (hornblende). 
$(\alpha=$ incolore; $\beta=$ vert; $\gamma=$ vert pâle, bleuté - fig. 26). Ces deux amphiboles se transforment encore en chlorite, parfois en talc et calcite. L'intensité des transformations est fonction de l'intensité des déformations qui ont affecté les roches.

La séricite est rarement présente, de même que la biotite brune.

L'ilménite, en taches parfois squelettiforme, est plus ou moins leucoxénisée.

Les roches gabbro-dioritiques sombres de l'unité moyenne sont schisteuses.

Sous le microscope, les minéraux sont très écrasés. Le plagioclase a une composition identique à celui qui se trouve dans les roches plus claires des autres unités, tandis que l'amphibole est une actinote un peu plus bleutée. Elle est très cataclasée et chloritisée. Les feuillets de chlorite sont disposés parallèlement à la direction de la schistosité.

Les pourcentages des différents constituants minéralogiques de ces roches sont donnés dans le tableau 9.

Tableau 9. Composition minéralogique des gabbro-diorites.

\begin{tabular}{|c|c|c|c|c|c|}
\hline \multirow[t]{2}{*}{$\mathrm{n}^{\circ}$ plaques GGU } & 32704 & 32703 & 32731 & 32792 & 54825 \\
\hline & $\%$ & $\%$ & $\%$ & $\%$ & $\%$ \\
\hline Plagioclase. & 30 & 20 & 50 & 43 & 48 \\
\hline Epidote............. & 10 & 20 & & & \\
\hline Amphibole..... & 52 & 40 & 41 & 50 & 46 \\
\hline Chlorite $\ldots \ldots \ldots \ldots \ldots \ldots$ & 15 & 15 & & & \\
\hline Ilménite et leucoxène . . . . & 3 & 5 & 9 & 7 & 6 \\
\hline
\end{tabular}

Les gabbro-diorites sont caractérisés par un pourcentage élevé des ferromagnésiens (amphibole, chlorite, ilménite) qui se situe autour de $60 \%$ et une disposition ophitique des lattes de plagioclase comme dans les gabbros et les diabases. Par contre, la composition du plagioclase $(30 \%$ d'An) et la présence d'amphibole brune sont plus typiques des diorites.

\section{Déformations des roches gabbro-dioritiques}

Les déformations intimes de ces roches débutent par une faible cataclase des amphiboles et des lattes de plagioclase. Dans les roches plus déformées, l'amphibole forme de petits yeux étirés et enveloppés de matériel feldspathique granulé. Finalement, la roche acquiert une schistosité assez bien marquée qui s'accompagne d'une forte chloritisation des amphiboles, d'une saussuritisation du plagioclase et d'une leucoxénisation complète de l'ilménite. La chlorite et le Jeucoxène se disposent parallèlement à la schistosité et on observe aussi une faible cristallisation d'actinote en fibres très tenues, parallèles elles aussi à cette schistosité. Ce type de 
déformation affecte surtout les roches gabbro-dioritiques de l'unité de transition et celles de l'unité moyenne. Dans les autres unités, les déformations importantes sont seulement localisées à certaines zones (p. 108).

\section{Mise en place des gabbro-diorites}

La mise en place des gabbro-diorites sous forme de filons-couches nous semble peu probable pour plusieurs raisons:

Aucune discordance, même locale, n'a pu être observée entre les coulées de pillows et les masses gabbro-dioritiques.

La couleur de ces gabbro-diorites est toujours semblable à celle des pillows dans chaque unité lithostratigraphique du Groupe d'Arsuk. La mise en place des gabbro-diorites est contemporaine de celle des pillows, car on retrouve des fragments de ces deux types de roche dans les brèches volcaniques remaniées intercalées à plusieurs niveaux dans l'édifice volcanique (p. 70).

L'absence de structure de refroidissement rapide dans la partie supérieure des masses gabbro-dioritique nous amène à considérer ces roches comme des coulées subaquatiques non en contact avec l'eau. Lors de l'éruption volcanique, une partie seulement de la lave émise s'est trouvée directement en contact avec l'eau (ce qui a donné naissance aux pillowlavas); la lav', continuant à être émise, se trouvait alors protégée du refroidissement par une "couverture » de pillows et pouvait ainsi se propager à des distances de plus en plus grandes des bouches volcaniques.

Des phénomènes identiques sont décrits par M. E. Wilson, au Canada (1942 et 1960, p. 101): "The presence or absence of pillows in part or all of a single lava flow or in different lava flows of the same chemical composition may be related to the intimate or non-intimate way in which the lava comes in contact with water, and this, in turn may have a relationship to one or all of three factors:

The size of the flow,

the temperature of the lava,

the speed of movement of the lava prior to its consolidation."

Le mode de mise en place des gabbro-diorites nous amène à comparer la composition pétrographique de ces roches avec celle des pillows. Dans les deux types de roche, les ferromagnésiens sont représentés par des amphiboles, des chlorites, de l'ilménite et plus rarement de la biotite. L'amphibole brune, caractéristique des gabbro-diorites, n'a pas été retrouvée dans les pillows. Elle a pu cependant former les petits phénocristaux (signalés p. 58) pseudomorphosés en actinote. Le contenu en ilménite des pillows est assez semblable à celui des gabbro-diorites. 
La composition du plagioclase est plus basique dans les gabbrodiorites que dans les pillows. Cette différence peut être liée à une saussuritisation plus rapide des microlites de plagioclase calcique dans les pillows ou bien elle peut refléter une variation de composition du plagioclase primaire: contamination de la lave des pillows par l'eau de mer riche en Na.

Si les textures des pillows sont assez différentes de celles des gabbrodiorites, on observe dans la partie centrale de gros pillows un arrangement des microlites de plagioclase qui se rapproche beaucoup de la texture subophitique observée en bordure des coulées massives.

\section{Les filons-couches basiques dans les formations sédimentaires}

\section{Caractères morphologiques}

Des roches basiques, de composition gabbro-dioritique, sont intercalées dans les formations sédimentaires. Elles apparaissent dans l'horizon des schistes sombres sur la petite presqu'île d'Inugsuk, près de Taylers Havn et sont bien développées dans la Formation d'Isua, sur les côtes Est et Nord d'Arsuk $\emptyset$. On en trouve également sur le petit îlot Kînâlik, au large d'Ikapynt.

Ces intercalations de roches basiques correspondent probablement à des filons-couches, bien qu'aucune preuve de discordance avec les séries sédimentaires encaissantes n'ait pu être établie. L'épaisseur des filonscouches est très variable. Sur la presqu'île d'Inugsuk, l'un d'entre eux a une dizaine de mètres d'épaisseur. Celui qui se trouve le long de la côte Nord d'Arsuk $\varnothing$ a $300 \mathrm{~m}$ d'épaisseur environ. Sur le versant Nord de l'île (au Sud de Qimatut, à l'altitude $200 \mathrm{~m}$ ), les phyllites de la Formation d'Isua renferment plusieurs petits filons-couches de 20 à $50 \mathrm{~cm}$ d'épaisseur qui sont boudinés.

Macroscopiquement, ces roches basiques sont caractérisées par un fort laminage des amphiboles qui forment de petits yeux ayant jusqu'à $5 \mathrm{~mm}$ de long sur $2 \mathrm{~mm}$ de large. Dans les zones plus déformées, ces yeux s'étirent et s'aplatissent. On passe alors à des schistes amphiboliques ou, par transformation, à des chloritoschistes.

\section{Composition pétrographique}

L'examen microscopique d'une roche intimement peu déformée révèle:

Un arrangement ophitique des lattes de plagioclase complétement saussuritisé. Ces lattes de $0,5 \mathrm{~mm}$ de long sont englobées dans de larges amphiboles vert pâle ou incolores, cataclasées et partiellement chloritisées. 
L'amphibole correspond à une actinote. Dans certains échantillons, cette amphibole renferme une tache légèrement brune qui pourrait correspondre à une relique d'amphibole brune.

Le quartz, peu abondant, est disposé sous forme de taches ou parfois en lits lenticulaires dus à une ségrégation (" quartz discordant et lamellaire"). L'ilménite leucoxénisée est toujours présente en faible quantité (jusqu'à $2 \%$ ) ainsi que l'apatite. La biotite brune n'a été reconnue qu'à Niaqornarssuaq, à la pointe Nord-Est d'Arsuk $\emptyset$ et sur la presqu'île d'Inugsuk; sa formation est postérieure à la cataclase principale de ces roches basiques.

Dans les roches très déformées, la texture ophitique n'est plus reconnaissable, car le plagioclase est très granulé. La composition de deux de ces grains de feldspath (GGU 32639) a pu être mesurée à la platine universelle et correspond à une andésine à $45 \%$ An $\left(2 \mathrm{~V} \alpha=100^{\circ}-96^{\circ}\right.$; $\alpha \mathrm{V} 010=22^{\circ}-23^{\circ}$, section perpendiculaire à $\gamma$; l'indice est supérieur ou égal à l'indice $\beta$ du quartz). Mais généralement la saussuritisation est trop avancée pour permettre une détermination, même approchée. Dans des roches déformées, l'actinote se déchire en fibres qui se disposent parallèlement à la direction d'étirement. Elle se transforme en chlorite ou, plus rarement, en talc. Cette transformation va jusqu'à la complète disparition de l'amphibole dont les reliques sont finalement pseudomorphosées en amas de chlorite. Sur la côte orientale de la presqu'île d'Inugsuk, ainsi que sur l'îlot Kînâlik, on observe une recristallisation d'amphibole vert pâle qui s'allonge parallèlement à la schistosité et entoure quelques plagioclases microgrenus (oligoclase).

\section{Relation entre les filons-couches et les laves massives gabbro-dioritiques}

La composition pétrographique des filons-couches basiques est très semblable à celle des laves massives extrusives du groupe volcanique. Il est probable qu'ils aient la même origine magmatique. Leur mise en place sous forme intrusive est certainement synchrone de la mise en place des formations volcaniques extrusives.

\section{Les filons basiques kétilidiens dans l'édifice volcanique}

\section{Caractères morphologiques}

Dans la région occidentale d'Arsuk $\varnothing$, on observe plusieurs filons de 0,5 à $2 \mathrm{~m}$ d'épaisseur, de direction sensiblement $\mathrm{N}-\mathrm{S}$, qui recoupent à la fois les coulées massives et les coulées en pillows. Ces filons n'ont pu être suivis que sur quelques centaines de mètres au maximum. Leur répartition cartographique semble correspondre à une disposition générale "en échelon ». Leur origine intrusive est attestée par la présence d'enclaves de gneiss albitique et de matériel quartzitique. Ces filons sont atteints par les mêmes déformations que celles qui ont affecté les roches 
du Groupe d'Arsuk. Ils ne sont jamais boudinés, ce qui montre déjà qu'au moment de leur déformation leur compétence devait être semblable à celle des pillows et des laves massives qu'ils recoupent. Compétence semblable, mais non identique, puisque la schistosité qui les affecte y est légèrement réfractée (Fou MMARIER, 1949).

Des filons de ce type sont visibles sur la côte au Nord-Ouest de Kangerdluk, ainsi qu'à Krabbenæs. D'autres se trouvent dans la région occidentale d'Hekseø et sur la côte Est de Manîtsoq, ainsi que sur la rive orientale du lac $490 \mathrm{~m}$. Aucun filon n'à été reconnu dans l'unité volcanique supérieure, ni dans les formations du groupe sédimentaire.

\section{Composition pétrographique}

De couleur vert clair ou vert sombre, ces filons sont caractérisés par la présence de phénocristaux très cataclasés d'amphibole verdâtre à noire, ayant jusqu'à $2 \mathrm{~cm}$ de diamètre.

Sous le microscope, on distingue des phénocristaux d'amphiboles brunes, pléochroïques $(\alpha=$ brun clair; $\beta=$ brun-roux; $\gamma=$ brun-orange). Ces amphiboles sont parfois verdâtres dans la partie centrale $(\alpha=$ vert-jaune un peu brun; $\beta$ et $\gamma=$ vert-olive brun). Elles sont transformées en actinote (incolore ou vert pâle) et sphène. Ces deux amphiboles sont finalement chloritisées.

Dans les roches les moins écrasées, les lattes de plagioclase, très saussuritisées et séricitisées, sont en disposition ophitique. Leur composition oscille entre l'albite et l'oligoclase. Il est possible que le plagioclase primaire ait été plus calcique car certaines lattes, très saussuritisées, semblent zonées et sont auréolées d'albite de néoformation. Les minéraux secondaires sont constitués par l'épidote, la calcite et le leucoxène.

Comme on peut s'en rendre compte, la composition de ces roches est assez semblable à celle des laves massives et des filons-couches gabbrodioritiques.

\section{Mode de mise en place et âge des filons amphibolitiques}

De par leur composition et leurs relations structurales avec les roches encaissantes, les filons amphibolitiques doivent appartenir au même volcanisme que celui qui a donné naissance aux laves et aux pillows du Groupe d'Arsuk. Leur âge est kétilidien. Ces filons correspondent peut-être à des «cheminées " latérales ouvertes lors d'une nouvelle phase de l'éruption.

\section{Les roches ultrabasiques}

Des masses de roches ultrabasiques sont intercalées entre les coulées volcaniques du Groupe d'Arsuk. A l'échelle de la carte géologique, elles semblent concordantes avec les roches encaissantes. Toutefois, les zones de contact sont trop laminées pour permettre de connaître exactement les relations structurales existant entre les masses ultrabasiques et les roches encaissantes. 


\section{Les péridotes à hornblende}

Des masses de 100 à $200 \mathrm{~m}$ de long et de $5 \mathrm{~m}$ d'épaisseur se trouvent à la base de l'importante coulée de lave gabbro-dioritique à l'Est de Kangerdluk, ainsi qu'à Fladedal.

Au microscope, elles sont constituées principalement de grains d'olivine serpentinisée, englobés dans de larges amphiboles brunes pléochroïques ( $\alpha=$ jaune-brun; $\beta=$ brun clair; $\gamma=$ brun-roux, fig. 26). Cette amphibole est partiellement transformée en une actinote incolore qui est finalement chloritisée. Le carbonate est toujours présent en faible quantité.

\section{Les serpentines}

Dans le région occidentale d'Arsuk $\emptyset$, à la base de l'unité supérieure, près de la charnière du grand pli d'Ørnevæggen, se trouve une intercalation de serpentine de $100 \mathrm{~m}$ de long et de $50 \mathrm{~cm}$ d'épaisseur environ. Elle est constituée d'antigorite, talc, carbonate et magnétite.

Sur le flane Est de Storpuklen se trouve une masse assez importante de roche ultrabasique (dunite métamorphisée) très déformée et transformée partiellement en serpentine verte. Au microscope, la roche est composée d'antigorite, talc et magnétite avec des reliques d'olivine.

\section{Les hornblendites et les talcschistes}

Dans la région Sud d'Arsuk $\varnothing$, en amont du ravin d'Ivît Elv, plusieurs niveaux ultrabasiques, de 10 à $20 \mathrm{~cm}$ d'épaisseur, sont intercalés entre les coulées de pillows et de lave massive. On distingue des hornblendites et des talcschistes. Le niveau inférieur est formé de hornblendite.

Sous le microscope, cette roche est consituée de hornblende brune, transformée partiellement en actinote, puis en chlorite. Les constantes optiques de ces deux amphiboles sont données dans la fig. 26 . Les minéraux secondaires sont l'ilménite, le carbonate ainsi que quelques grains isolés de feldspath.

Une autre intercalation de hornblendite, constituée d'actinote plus ou moins chloritisée et d'ilménite leucoxénisée, se trouve à quelque $100 \mathrm{~m}$ au-dessus de la précédente et se poursuit jusqu'à la côte Sud, à l'Ouest d'Umingmait. Dans le ravin d'Ivît Elv se trouvent encore quelques intercalations de talcschistes chloritotrémolitiques, plus ou moins riches en carbonate, contenant parfois de la magnétite et qui proviennent sans doute de la transformation de roche ultrabasique (HARKER, 1960).

\section{Les déformations des ultrabasites}

Les roches ultrabasiques sont très déformées. Dans la région d'Ivît Elv, elles sont plissées comme les roches volcaniques encaissantes. Dans la région de Kangerdluk et de Fladedal, les péridotites sont fortement laminées et sont débitées en grosses lentilles à surfaces serpentinisées. 
Relation entre les ultrabasites et les roches volcaniques basiques

De par leur composition pétrographique et leur disposition structurale, les roches ultrabasiques d'Arsuk $\varnothing$ pourraient appartenir au même volcanisme que celui qui a donné naissance aux pillows et aux laves massives. Par différenciation magmatique, on aurait une série continue allant des dunites aux gabbro-diorites en passant par les péridotites à hornblende brune. Les relations structurales existant entre les ultrabasites et les roches basiques encaissantes ne sont toutefois pas assez claires pour le prouver et la possibilité d'une mise en place des roches ultrabasiques, sous forme de filons-couches s'intercalant entre les séries volcaniques avant ou lors des premiers stades de leur déformation, n'est pas exclue.

\section{Le matériel sédimentaire et siliceux dans le Groupe d'Arsuk}

Par rapport à l'énorme quantité de produits volcaniques composant le Groupe d'Arsuk, le matériel sédimentaire et siliceux est pratiquement négligeable. Au point de vue tectonique cependant, les minces niveaux sédimentaires prennent une importance considérable en permettant de suivre le déroulement des structures dans des formations volcaniques assez monotones. On distingue des phyllites et des quartzites ou des cherts.

\section{Les phyllites pyriteux avec cherts rubanés et lenticulaires}

Sur Arsuk $\varnothing$, le contact entre l'unité de transition et l'unité inférieure du groupe volcanique est constitué par un horizon de phyllites rubanés, souvent très pyriteux, dans lequel il y a des lits lenticulaires de roche quartzitique. Cet horizon se retrouve à Storø, comme nous l'avons déjà indiqué (p. 46).

Par déformation tectonique, l'épaisseur de cet horizon varie beaucoup d'un endroit à l'autre. Au Nord-Est d'Arsuk $\varnothing$, il mesure localement $80 \mathrm{~m}$ (Nord de Domf jeld), tandis que dans la région occidentale, à Mitdlûvfik, son épaisseur, qui ne dépasse pas $20 \mathrm{~m}$, correspond encore à un empilement de couches repliées sur elles-mêmes.

Dans les secteurs les moins déformés, on observe une alternance de lits chloritoamphiboliques carbonatés de 3 à $6 \mathrm{~cm}$ d'épaisseur et de lits sensiblement de même épaisseur constitués de roche quartzitique beige clair, rubanée, souvent en lentilles (fig. 27). Dans un mème lit, les lentilles quartzitiques, de 10 à $50 \mathrm{~cm}$ de long, peuvent être disposées côte à côte à la manière d'une tablette de chocolat dont les carrés seraient séparés les uns des autres. Ce faciès correspond aux "quartzitic slabs" de Wegmann (1938, p. 20, fig. 5). Associés à ces roches se trouvent encore des phyllites finement rubanés chloritoquartzeux à grenats, souvent pyriteux et graphiteux. 


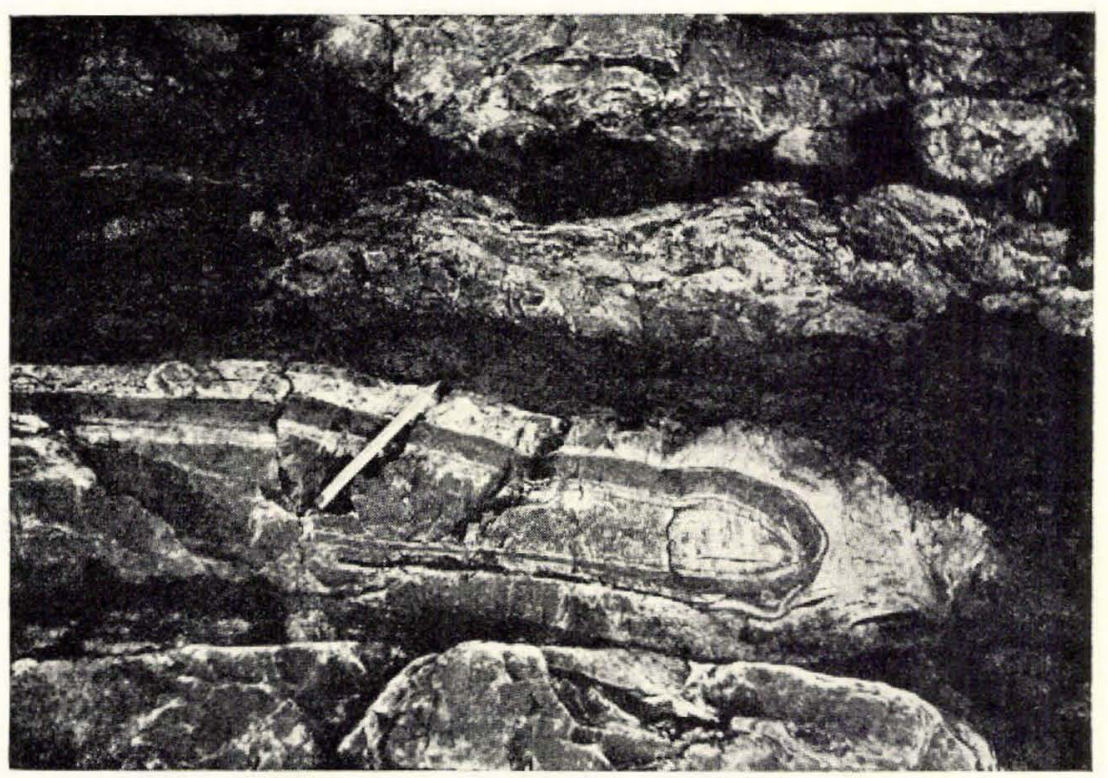

Fig. 27. Horizon de phyllites avec cherts lenticulaires intercalés dans les formations volcaniques à la base du Groupe d'Arsuk. Takisũnguaq, à l'Est de Mitdlûvfik. Les lentilles de chert contiennent de la magnétite qui se dispose en lits, épousant les bords des lentilles (ségrégation).

L'examen microscopique des roches quartzitiques montre que le quartz est en plages lobées équidimensionnelles de $0,05 \mathrm{~mm}$ de diamètre. On distingue quelques rhomboèdres de dolomie et un peu de minerai de fer. Le rubanement de certaines lentilles est dû à une recristallisation complète du quartz et à une ségrégation différentielle de minerai (magnétite) qui suit sensiblement les contours arrondis des extrémités des lentilles. Les rhomboèdres de dolomie peuvent s'aligner de la même manière. Par dissolution de ce carbonate, une multitude de petits trous "d'épingle " apparaissent à la surface de la roche. Dans les zones les plus carbonatées, un fort développement de trémolite fibro-radiée parvient à voiler complètement les structures. Dans les lits chloritoamphiboliques carbonatés, la trémolite est aussi abondante et forme de longues fibres ou des gerbes.

Par comparaison avec des formations de chert intercalées dans des roches volcaniques décrites dans la littérature (SAmpson, 1923; Williams et al., 1955), les roches quartzitiques contenues dans cet horizon de phyllites pyriteux correspondent sans doute à des cherts totalement recristallisés.

\section{Les grosses lentilles quartzitiques}

Des lentilles de matériel quartzeux, de couleur blanche brune, grisbleu, noire et rouillée sont intercalées en de nombreux endroits entre les coulées de pillow-lavas. Elles se disposent en "chapelet à la base et au sommet de l'unité moyenne du Groupe d'Arsuk. Ces lentilles, visiblement 
laminées sur les bords, peuvent atteindre une trentaine de mètres de long sur 5 à $6 \mathrm{~m}$ d'épaisseur, notamment à Manîtsoq, à Ørnevæggen et en amont du ravin d'Ivît Elv. Leur composition pétrographique est tout à fait identique à celle du matériel quartzeux qui se trouve entre les pillows (p. 60). La magnétite y est souvent plus abondante. Par ségrégation lors de la recristallisation du quartz, le minerai de fer peut se concentrer en lits de 1 à $2 \mathrm{~cm}$ d'épaisseur, parallèles aux bords des lentilles.

WeGmann $(1938$, p. 20) souligne que ces roches quartzitiques ont un "caractère différent" des quartzites du groupe sédimentaire. En effet, elles ne présentent aucune structure qui rappelerait de loin un litage et ne renferment pas de minéraux détritiques. Ces roches pourraient avoir une origine hydrothermale (anciens filons de quartz subconcordants déformés et boudinés) ou, peut-être, correspondre à des accumulations lenticulaires de matériel siliceux (chert) partiellement remobilisé.

\section{L'origine des cherts du Groupe volcanique d'Arsuk}

Les travaux modernes sur les silicifications ont montré que la silice se trouve dispersée dans l'eau sous forme de monomolécule $\mathrm{Si}(\mathrm{OH})_{4}$ (solution vraie), jusqu'à une teneur totale en silice inférieure à 100-140 ppm (à $25^{\circ} \mathrm{C}$ et pour un pH 9). Pour Millot (1964), les cherts sont le produit de la croissance des cristaux de silice (ou de germes) à partir des tétraèdres $\mathrm{Si}(\mathrm{OH})_{4}$ en liberté dans l'eau. Ce processus est évidemment très lent. Si la teneur en silice est supérieure à $140 \mathrm{ppm}\left(\grave{\mathrm{à}} 25^{\circ} \mathrm{C}\right.$ et $\left.\mathrm{pH} 9\right)$, l'excès de silice formera des polymères dont la présence caractérise les solutions colloïdales. Dunbar \& Rodgers (1957) expliquent la formation des cherts par sursaturation en silice des solutions aboutissant à la formation d'un gel de silicate hydraté. Dans ces deux hypothèses, on est obligé d'admettre un apport extérieur important de silice, car la teneur en silice de l'eau de mer varie entre 0,1 et $10 \mathrm{ppm}$ et celle des rivières entre 3 et 50 ppm (Millot, 1964; Clarke, 1924, cité par Dunbar \& RODGERS).

Davis (1918, cité par Taliaferro) y voit des «siliceous springs》. Pour Dunbar \& Rodgers (1957), la silice peut provenir de l'eau des geysers: 762 ppm pour l'Opal Spring de Yellowstone Park. De son côté, Taliaferro (1943) pense qu'un important apport de silice résulterait de l'interaction de la lave chaude et de l'eau de mer.

Dans le cas des cherts du Groupe d'Arsuk, l'apport de silice pourrait donc être lié au volcanisme kétilidien. Si le matériel quartzitique, intercalé entre les deux pillows visibles sur la planche 5, correspond à un chert et non à un matériel détritique, sa formation doit s'expliquer par précipitation directe à partir de solution sursaturée. Ce phénomène devrait être suffisamment rapide pour que le chert déjà consolidé puisse être déformé par le poids du pillow supérieur. 
La présence de minerai de fer, localement abondant, dans ces cherts serait aussi en relation avec les phénomènes volcaniques ayant donné naissance aux pillows, laves massives, tufs, etc, ainsi que le note TALIAFERRo (1943, p. 152) "there is nothing new in the idea of the discharge of iron and manganese compounds from volcanic springs, either subaerial or submarine ».

Néanmoins, pour certains cherts nous scmmes obligés d'admettre un apport extérieur détritique, dont nous ne connaissons pas l'importance, car nous avons trouvé quelques zircons arrondis dans un chert noir situé sur l'îlot Mitdlûvfik, juste en dessous de l'horizon de chert rubané et lenticulaire.

\section{Les gneiss du vieux socle}

De par la position insulaire de la région d'Arsuk, le contact entre les roches supracrustales et les gneiss du vieux socle n'est visible que sur une courte distance. Partout où il est observable, ce contact est de nature tectonique. Dans les zones de contact, les roches sont souvent très écrasées sur une épaisseur variant de quelques décimètres à plusieurs mètres. En dehors de ces zones, les roches peu métamorphiques kétilidiennes ont pu être distinguées assez facilement des roches gneissiques ou amphibolitiques, ne serait-ce que par leur couleur et texture.

Le socle autour d'Arsuk $\varnothing$ est constitué de roches métamorphiques variées appartenant à plusieurs séries lithologiques. L'étude de ces roches dépassant le cadre de ce travail, nous nous arrêterons à la description de quelques échantillons de gneiss et amphibolites prélevés à proximité du contact et qui appartiennent à une même série appelée "série d'Ivigtut " (Berthelsen, 1958). En s'écartant du contact, une autre série, dite des "gabbro-anorthosites ", succède à la série d'Ivigtut. Enfin, des fragments de roches gneissiques, situées sous les séries kétilidiennes d'Arsuk, et "hissées » à la surface du sol par la lave de certains filons volcaniques Gardar ou post-Gardar, ont été également examinés.

\section{Les gneiss d'Ivigtut}

\section{Les gneiss de la côte Nord d'Arsuk $\varnothing$}

Sur la côte Nord d'Arsuk $\varnothing$, la juxtaposition de roches typiquement gneissiques avec des roches macroscopiquement moins transformées, appartenant à la Formation de Taylers Havn, se fait par l'intermédiaire de zones mylonitiques.

L'examen microscopique de gneiss foliés montre que ces roches sont constituées principalement de matériel quartzofeldspathique très granulé, de biotite et d'épidote et accessoirement de zoïsite, sphène, muscovite, chlorite, trémolite, minerai de fer, apatite, zircon arrondi et tourmaline tardive. 
Les plagioclases, dont la composition varie entre l'albite et l'oligoclase, sont plus ou moins séricitisés. Ils peuvent atteindre $3 \mathrm{~mm}$ de diamètre et sont cassés, tordus ou pulvérisés. Il n'y a pas de feldspath potassique.

La zoïsite, en larges cristaux (jusqu'à 1,5 mm de diamètre), est aussi cassée, de même que le sphène, tandis que les feuillets de muscovite et de chlorite se disposent suivant la foliation.

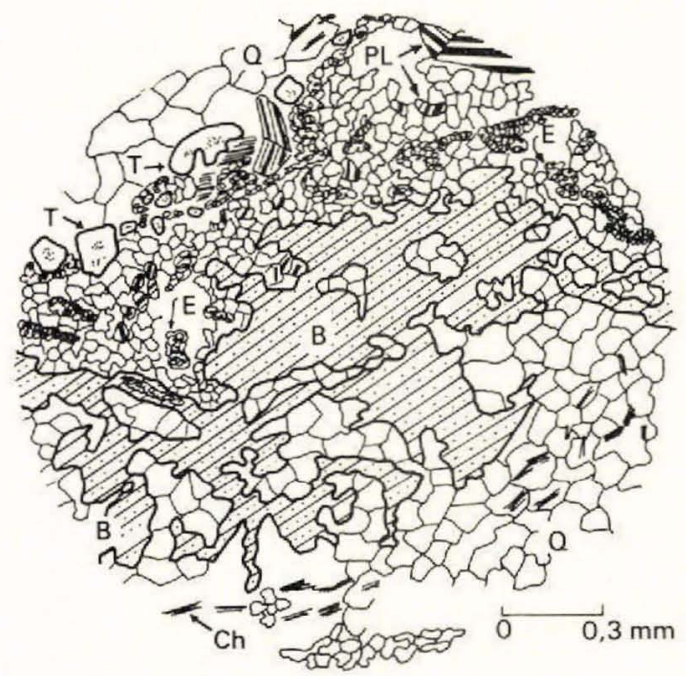

Fig. 28. Micrographie d'un gneiss à biotite de la côte Nord d'Arsuk Ø. Le quartz (Q) et le feldspath (PL) sont très cataclasés. Le quartz est partiellement recristallisé. Développement de larges biotites brunes (B) poecilitiques, postérieures à la cataclase principale. La formation de l'épidote (E) est postérieure à celle de la biotite. La tourmaline (T) est postérieure à tous les minéraux qu'elle renferme parfois en inclusions (échantillon GGU 48820).

La biotite brune se développe en larges taches poecilitiques (fig. 28) et s'infiltre dans les fissures des minéraux précédents. Elle est nettement postérieure à la cataclase principale des gneiss, mais a subi une nouvelle déformation (torsion des feuillets et parfois granulation).

La trémolite se développe parfois poecilitiquement. Elle est cataclasée comme la biotite.

L'épidote (pistacite) est généralement en très petits grains qui parfois innondent la roche en corrodant la zoïsite et s'infiltrent dans les fissures du sphène. Cette épidote peut ressouder d'anciens fragments cataclasés de clinozoïsite ou d'orthite. Sa formation tardive est postérieure à celle de la biotite.

Les paragenèses observées dans ces gneiss sont les suivantes:

quartz-plagioclase-zoïsite-sphène-ilménite-apatite

cataclase principale

trímolite-biotite puis épidote (pistacite)

cataclase peu marquée

tourmaline

Ces gneiss proviennent sans doute de la transformation de roches pélitiques. 
Les gneiss et les amphibolites de l'îlot Narssap sagdlia

Les gneiss sombres rubanés de l'îlot Narssap sagdlia, au large d'Ikapynt, sont caractérisés par une alternance irrégulière de lits leucocrates et mélanocrates. Ils contiennent parfois de nombreux porphyroblastes de feldspath, pouvant atteindre $1 \mathrm{~cm}$ de diamètre, donnant à la roche une structure oeillée. Ces roches sont vraisemblablement des produits de transformation de roches basiques amphiboliques.

Les lits leucocrates sont constitués de feldspaths (potassiques et plagioclases), de biotite et accessoirement de zoïsite, orthite et actinote.

Le plagioclase (albite et oligoclase) est, en quantité et en grosseur,iden tique au feldspath potassique, mais il est toujours plus cataclasé que ce dernier (Belliere, 1958, p. 159). Le feldspath potassique contient de nombreuses inclusions désorientées de plagioclase qu'il semble corroder. Les myrmékites sont fréquentes dans les plagioclases en contact ou inclus dans le microcline. Ces myrmékites semblent être à leur tour corrodées par le feldspath potassique qui contient de petits vermicules de quartz (BELliere, 1958, fig. 9). La saussuritisation et la séricitisation atteint de préférence le feldspath calcosodique. Les gros feldspaths sont cassés et granulés sur les bords et sont entourés d'un mélange quartzofeldspathique extrêmement fin.

La biotite vert olive, parfois poecilitique, se développe dans la matrice finement granulée ou bien sur des feldspaths cataclasés. Elle a subi ensuite les effets d'une nouvelle cataclase et ses fragments se disposent parallèlement à la foliation de la roche.

Les lits mélanocrates sont formés principalement d'actinote et biotite et accessoirement de zoĩsite, ilménite leucoxénisée, quartz et feldspath.

L'actinote, vert bleuté, largement biotitisée avec apparition d'épidote (pistacite) et de sphène, n'est certainement pas primaire. Elle provient sans doute de la transformation d'un autre minéral (une autre amphibole?), car elle renferme des grains de sphène et du quartz d'exudation. Le pléochroïsme de cette actinote est identique à celui de l'actinote $A_{2}$ contenu dans les amphibolites décrites ci-après (tableau 10).

Les amphibolites qui se trouvent sur le même îlot sont constituées principalement d'amphibole et de plagioclase et accessoirement de quartz,

Tableau 10. Comparaison du pléochroüsme des amphiboles dans les gabbros métamorphiques et les gneiss à amphibole de Narssap sagdlia.

\begin{tabular}{|c|c|c|c|}
\hline & \multicolumn{2}{|c|}{$\begin{array}{c}\text { Gabbro métamorphique } \\
\qquad(38871)\end{array}$} & \multirow{2}{*}{$\begin{array}{c}\text { Gneiss rubanés } \\
(38866) \\
\text { Amphibole }\end{array}$} \\
\hline & Amphibole $A_{1}$ & Amphibole $A_{2}$ & \\
\hline $\begin{array}{l}\alpha \ldots \ldots \ldots \ldots \ldots \ldots \ldots \\
\beta \ldots \ldots \ldots \ldots \ldots \ldots \ldots \\
\gamma \ldots \ldots \ldots \ldots \ldots \ldots\end{array}$ & $\begin{array}{c}\text { jaune-brun clair } \\
\text { vert foncé } \\
\text { brun-vert olive }\end{array}$ & $\begin{array}{c}\text { jaune } \\
\text { vert } \\
\text { vert bleuté }\end{array}$ & $\begin{array}{c}\text { jaune } \\
\text { vert } \\
\text { vert bleuté }\end{array}$ \\
\hline
\end{tabular}


ilménite leucoxénisée et zoïsite. Les roches les moins déformées montrent une texture ophitique.

Les lattes de plagioclase, zonées de l'oligoclase à l'andésine $(40 \%$ d'An), sont séricitisées et transformées sur les bords en albite. Elles sont partiellement englobées par de larges amphiboles vertes.

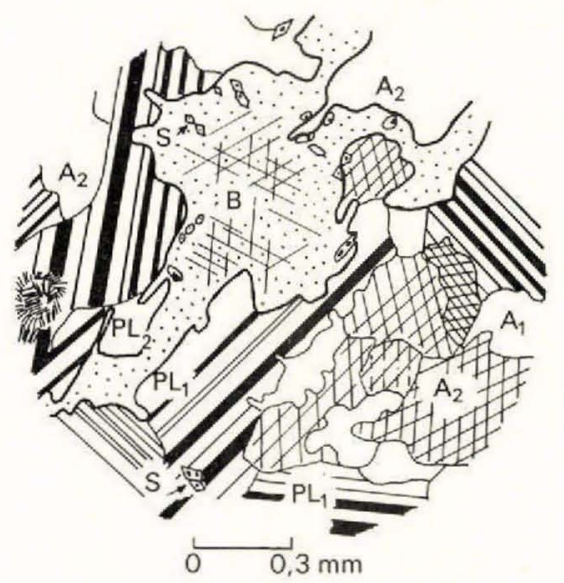

Fig. 29. Micrographie d'une amphibolite de Narssap sagdlia. L'hornblende $A_{1}$ se transforme en actinote $A_{2}$. Ces deux amphiboles sont transformées en biotite brune (B), poecilitique, avec formation de sphène (S), idiomorphe, et rutile.Texture ophitique préservée. Néoformation d'albite $\left(\mathrm{PL}_{2}\right)$, en bordure du plagioclase maclé $\left(\mathrm{PL}_{1}\right)$.

Une hornblende $A_{1}$, pléochroïque, chargée d'inclusions opaques, se transforme en actinote $A_{2}$ vert pâle (fig. 29). Ces deux amphiboles sont partiellement transformées en biotite brune chargée d'agrégats de sphène et d'aiguilles de sagénite.

Bien qu'aucun pyroxène n'ait èté reconnu dans ces amphibolites, les inclusions allongées et disposées orthogonalement dans les hornblendes $A_{1}$ pourraient provenir de la transformation d'un pyroxène (inclusions de Schiller?).

Les paragenèses de cette amphibolite sont les suivantes:

hornblende verte, andésine, ilménite

actinote, biotite, sagénite, chlorite, albite, sphène

\section{Les gneiss de la côte Nord-Ouest de Tavdlorutit}

Les gneiss homogènes qui constituent la partie Nord-Ouest de l'ile Tavdlorutit passent, dans le secteur d'Avalagiaq, à des gneiss agmatitiques. La couleur sombre des «enclaves " est due à la présence de biotite.

$\mathrm{Au}$ microscope, les enclaves sont formées principalement de quartz et de plagioclase très saussuritisé qui représente les $3 / 4$ du volume de la roche. Les minéraux mafiques sont représentés par la biotite verte, l'épidote et le sphène. 
Dans le matériel leucocrate, ces minéraux sombres sont pratiquement absents. Les plagioclases sont remplacés partiellement par le microcline. Les myrmékites sont rares. Les figures de cataclase de ces roches sont identiques à celles qu'on observe dans les gneiss de Narssap sagdlia.

\section{Les gneiss entre Taylers Havn et Taylers Fjeld}

Les quartzites de la base de la Formation de Taylers Havn reposent, entre Bjørneelv et Taylers Fjeld, sur des roches écrasées parmi lesquelles on distingue des gneiss rubanés, des gneiss plus ou moins oeillés et des gneiss homogènes recoupés en tous sens par des veines de composition granitique.

Au microscope, on observe tous les intermédiaires entre une roche mylonitique où les fragments quartzofeldspathiques ont un diamètre voisin de 0,05 à $0,1 \mathrm{~mm}$ et une roche moyennement écrasée, du type de celle de Narssap sagdlia ou d'Avalagiaq. La composition minéralogique de quelques gneiss de Taylers Havn est donnée sur la fig. 7.

Au contact entre les gneiss et les quartzites se trouvent parfois des roches phylliteuses quartzofeldspathiques qui portent les traces d'un

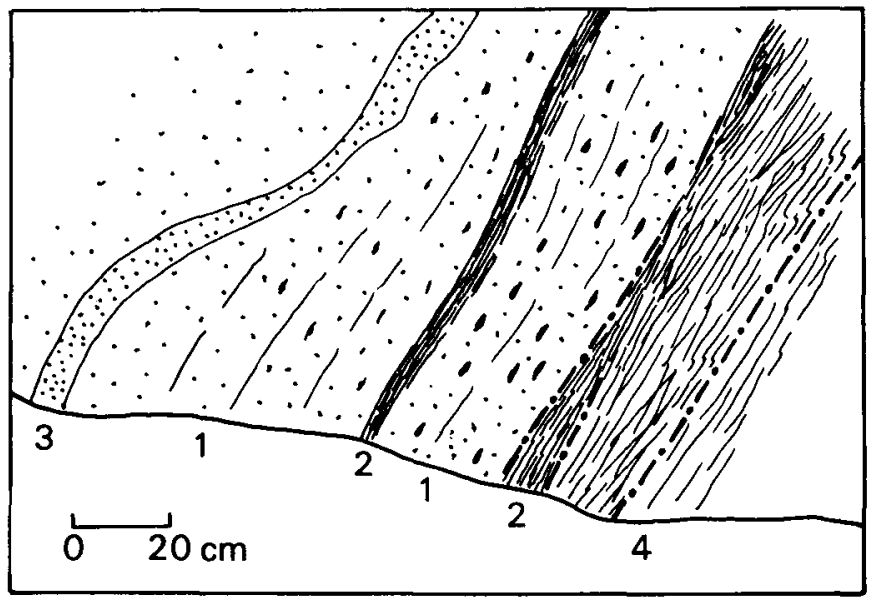

Fig. 30. Contact tectonique entre les gneiss prékétilidiens et les quartzites kétilidiens au Nord de Bjørneelv. 1: quartzites feldspathiques. 2 : phyllonites. 3 : veine de quartz.

4: gneiss.

fort écrasement et qu'il est difficile de distinguer pétrographiquement des gneiss mylonitisés. Ces roches, qui correspondent à des phyllonites, ont été reconnues immédiatement au Nord de Bjørneelv où elles forment une intercalation d'une dizaine de centimètres d'épaisseur, pincée entre les quartzites et les gneiss (fig. 30). On en retrouve également dans la région de Taylers Fjeld à l'altitude de $125 \mathrm{~m}$, où elles ont une épaisseur de 30 à $50 \mathrm{~cm}$. 
Ces phyllonites sont parfois constituées presque exclusivement de séricite $(70 \%)$. Dans d'autres roches de ce type, on reconnait au microscope une alternance de lits quartzofeldspathiques et de lits très sériciteux. La biotite verte est peu abondante ou absente. Le feldspath, principalement calcosodique, est fortement granulé. Le quartz est recristallisé "lamellairement ». En se basant uniquement sur la composition minéralogique de ces roches, il est évidemment impossible de savoir si elles proviennent de l'écrasement de gneiss ou bien de roches phylliteuses du type de celles qui se trouvent dans l'horizon d'Evqitsut (p. 19).

\section{Les gneiss avec enclaves gabbro-anorthositiques}

Pour donner un inventaire plus complet des différentes roches qui constituent le vieux socle, fournisseur probable des matériaux détritiques kétilidiens, il nous a paru intéressant de donner une description sommaire des gneiss gabbro-anorthositiques qui constituent un horizon repaire suivi sur une grande distance entre la région de Târtoq, au NW d'Arsuk $\varnothing$, et celle d'Íka, au SE. Ils affleurent sur la plus grande partie de la péninsule au Nord d'Arsuk $\varnothing$. Ils sont séparés des quartzites de la Formation de Taylers Havn, au Nord du détroit d'Ikerasârssuk, par un mince liseré de gneiss appartenant à la série d'Ivigtut.

Les renseignements pétrographiques que nous rapportons ici sont empruntés à la littérature. Berthelsen donne en 1960 une description trés sommaire des caractères de ces "gabbro-anorthosites», dans lesquels se trouvent des enclaves abondantes, des boudins ou des bancs de roches à plagioclase calcique et hornblende. Selon Henriksen (1961), les enclaves montrent toutes les transitions entre une roche gabbro-anorthositique et une ultrabasite. La composition du plagioclase varie entre 50 et $70 \%$ d'anorthite dans les roches peu recristallisées et 40 à $50 \%$ d'anorthite lorsque la recristallisation est plus avancée. La quantité de feldspath et d'hornblende varie beaucoup d'une enclave à l'autre. WindLEY (1967) rattache génétiquement les gabbro-anorthosites de la région d'Ivigtut aux anorthosites "stratifiées " de Fiskenæsset (à $130 \mathrm{~km}$ au Nord de Frederikshåb). Selon cet auteur, ces anorthosites stratifiées contenant de la chromite se seraient formées par différenciation gravitative dans des conditions stables préorogéniques.

\section{Les gne:ss situés sous les Formations d'Arsuk Ø}

Les filons volcaniques subverticaux, qui traversent les roches supracrustales d'Arsuk $\emptyset$, contiennent fréquemment des xénolithes de roches arrachées aux épontes et qui furent emportées vers le haut par le courant de lave (fig. 31). Les xénolithes sont très abondants dans certains filons 


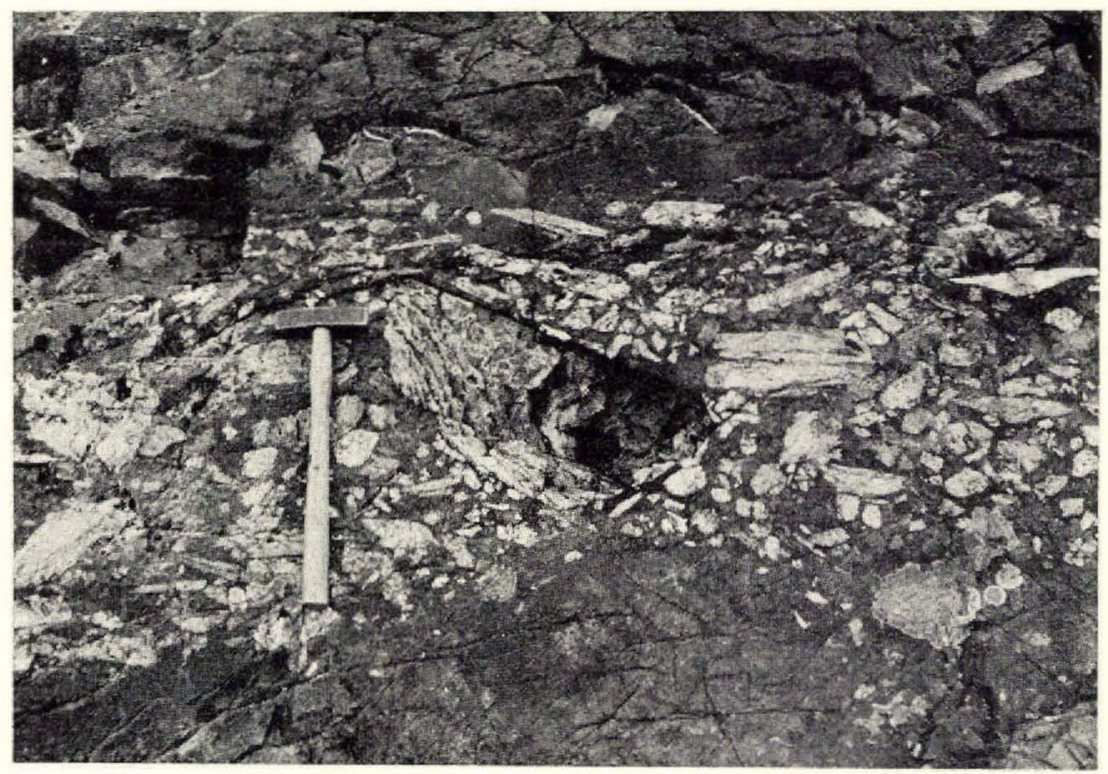

Fig. 31. Filon basique d'âge Gardar. Pinguligssuánguaq, Nord d'Arsuk Ø. Xénolithes de gneiss, diorites, quartzites (à droite du fer du marteau) et de roches vertes.

Gardar et post-Gardar de l'îlot Mitdlûvfik et de la pointe Nord-Ouest d'Arsuk $\varnothing$. On en trouve aussi dans les petits filons kétilidiens à l'Est de Pinguligssuánguaq. Les xénolithes sont constitués principalement de

gneiss leucocrates homogènes, de diorites quartzifères et de diorites à texture grenue dont les minéraux ne sont pas cataclasés, de quartzites massifs ou feuilletés très semblables à ceux de Taylers Havn,

de roches vertes compactes provenant probablement de la base du groupe volcanique.

\section{Les filons basiques discordants dans le vieux socle}

Les structures des gneiss dans le vieux socle sont recoupées par des filons basiques métamorphisés en amphibolites (amphibolites discordantes ou AD; p. 11). Ces filons sont très nombreux au NW et au SE d'Arsuk Fjord (Berthelsen, 1962). Dans la région d'Arsuk $\varnothing$, aucun d'eux n'a été observé à proximité du contact avec les roches supracrustales kétilidiennes. Les premiers filons apparaissent à quelque $500 \mathrm{~m}$ au Nord du détroit d'Ikerasârssuk et à plus de $1 \mathrm{~km}$ à l'Est de Taylers Havn.

Cinq générations de filons ont été mises en évidence par BoNDESEN \& Henriksen (1965) dans la région située au Nord d'Arsuk Ø. Ces filons 
correspondent à d'anciennes dolérites à olivine: pseudomorphoses d'augite et d'olivine, disposition ophitique des lattes de plagioclase zoné de l'andésine au labrador.

La transformation métamorphique et la déformation de ces filons augmentent progressivement du Nord-Ouest (îles de Sermersût et de Tôrnârssuk) vers le Sud-Est, en direction de la péninsule d'Ivigtut où ils sont même plissés (Berthelsen, 1962). Le métamorphisme des dolérites passe du faciès des schistes verts ou du faciès de l'albite-épidote-amphibole, dans les îles de Sermersût et de Tôrnârssuk, au faciès de l'amphibole sur la péninsule au Nord d'Arsuk Ø. Sur la péninsule d'Ivigtut, certains filons sont granitisés.

Primitivement, Berthelsen (1960 et 1961) pensait que la mise en place de ces dolérites était postérieure aux déformations kétilidiennes et que la transformation des dolérites en amphibolites était due au métamorphisme sanérutien.

Les travaux récents (WINDLEY et al., 1966) ont montré qu'il fallait modifier cette chronologie, du moins dans la région située au Nord d'Arsuk Fjord où le vieux socle a été reconnu avec certitude.

Si la plupart des filons doléritiques n'apparaissent que dans le socle de cette région, il en existe cependant quelques-uns qui recoupent également les sédiments plus jeunes et qui ont été déformés lors de la deuxième phase kétilidienne. Il est dif ficile de dater chronologiquement les autres filons qui ne se trouvent que dans le socle. Certains d'entre eux, à proximité de la discordance entre le vieux socle et les supracrustales, ont été déformés par des mouvements de chevauchement kétilidiens. Deux faits nous semblent importants:

l'épaisseur et le nombre des filons diminuent très rapidement dès qu'on s'approche de la discordance

aucun filon n'est recoupé par la surface de discordance.

Pour expliquer cette distribution si particulière de la plupart des filons doléritiques dans la région de Grænseland-Midternæs et d'Arsuk, nous pensons que les roches supracrustales devaient être déjà présentes lors de la mise en place des filons. On peut invoquer une différence de compétence entre le socle et la série supracrustale lors d'une phase d'extension caractérisant le début du dépôt des roches supracrustales. Ou bien, dans une variante, on peut songer aux anciennes fractures qui ont divisé le vieux socle avant la sédimentation des roches kétilidiennes.

Certains essaims de fracture ont pu se réouvrir au Kétilidien, mais la masse des sédiments qui les cicatrisait n'a pas suivi sauf dans de rares exceptions.

WeGMans (1938) pensait que certains filons visibles dans le socle auraient pu jouer le rôle de "canaux nourrisseurs " des coulées volcaniques 
kétilidiennes. Mais aucune connection entre filons et coulées n'a été observée jusqu'à maintenant et la composition pétrographique originale et de rétromorphose des filons doléritiques reste assez différente de celle des laves massives et des petits filons du Groupe d'Arsuk.

\section{Résumé}

La différence entre les gneiss d'Ivigtut et les roches supracrustales $\mathrm{du}$ bassin d'Arsuk est plus frappante sur le terrain car les structures typiquement migmatitiques sont généralement conservées malgré un écrasement intense des minéraux des roches. On reconnaît ainsi des gneiss rubanés, des gneiss homogènes ou foliés et linés, des gneiss veinés, des gneiss agmatitiques et des amphibolites provenant de la transformation (migmatisation) de roches pélitiques, semi-pélitiques et gabbroïques.

$\mathrm{Au}$-dessus de ces gneiss et en discordance tectonique se trouvent des roches métamorphiques qui n'ont pas été migmatisées (quartzites, phyllites, schistes, calcaires dolomitiques et produits volcaniques) et dans lesquelles les structures de dépôt sont parfois reconnaissables, sauf à proximité de la discordance où elles ont été masquées du fait des déformations et transformations kétilidiennes.

\section{Les minéraux détritiques dans les gneiss et les roches supracrustales}

Quelques minéraux lourds détritiques, examinés dans les gneiss et les roches supracrustales, ont attiré notre attention.

Les zircons. L'examen d'une centaine de plaques minces dans les roches sédimentaires et d'une vingtaine dans les gneiss a permis de mettre en évidence deux types de zircon.

Des zircons I, bien émoussés, arrondis ou ovales, se rencontrent dans les gneiss du socle et dans les sédiments plus jeunes. Ils semblent à première vue plus gros dans les gneiss que dans les sédiments. Ces zircons I sont d'origine détritique. Ils ont subi une abrasion intense pour acquérir leur forme émoussée.

Un autre type de zircon II n'a été observé que dans les quartzites kétilidiens de Taylers Havn. Ce sont des cristaux subidiomorphes ou même idiomorphes qui renferment parfois un noyau désorienté de zircon arrondi (qui pourrait être un zircon I). Ils sont partiellement cassés, mais leur abrasion est faible comparativement aux zircons I. Ces zircons ont pu se former soit dans les sables ou les grès kétilidiens par cristallisation authigénique (Butterfield, 1936; Krynine，1946; Vitanage，1957), soit dans les gneiss lors de transformations métamorphiques prékétili- 
diennes. Dans les deux cas, les zircons II ont été repris dans un cycle d'abrasion kétilidien.

Les tourmalines et les zoïsites. Les tourmalines sont très abondantes tant dans les roches prékétilidiennes que kétilidiennes. La plupart sont liées à une minéralisation tardi-kétilidienne. Dans certains cas, on peut reconnaître un noyau détritique (?)arrondi, en discontinuité optique, dans des tourmalines fraîches idiomorphiques (KRYNINE, 1946). Les zoïsites détritiques sont fréquentes; elles sont arrondies ou ovales, incolores ou chargées d'impuretés opaques. 


\section{TECTONIQUE}

\section{Introduction}

Les roches sédimentaires et volcaniques de la région d'Arsuk $\emptyset$ qui font partie d'un bassin sédimentaire kétilidien ont été déformées à plusieurs reprises durant l'activité orogénique kétilidienne. La tectonique liée à cette orogénèse est caractérisée par une superposition de structures plissées. Il s'y ajoute des chevauchements, cisaillements et fractures. Ces déformations ont donné naissance à la grande structure synclinale ou en «baignoire " de la région d'Arsuk. Nous avons comparé la structure de l'édifice volcanique à celle d'une "baignoire " évasée vers l'Est et dont les flancs ont une orientation ENE-WSW.

Vers le Sud-Ouest, les structures sont mal connues, mais il est probable que cette baignoire s'ouvre largement vers Storø.

Les déformations postérieures, d'âge Gardar et post-Gardar, sont de nature essentiellement cassante. Elles n'ont pas modifié considérablement les structures précédentes, mais leur rôle est primordial dans l'évolution morphologique du pays.

Dans ce chapitre, nous étudierons successivement:

les déformations kétilidiennes,

les déformations cassantes d'âge Gardar et post-Gardar.

La superposition des structures kétilidiennes a été reconnue pour la première fois par WeGMaNN (1938, p. 51, fig. 26 et 27):

"The greenstones of Arsuk Island are situated in a kind of syncline whose axis rises towards the east ... The content of the syncline shows no quite simple structure. The individual larger greenstone horizons especially where interbedded in the schists, have slid across one another, so that the whole mass becomes a somewhat complicated system of folds and overthrusts. It is all the more complicated because the thrusts have not only taken place across the axis of the syncline, but also in some degree lengthwise. At Taylers Havn, the upper schists have become folded and sheared in two directions whose axes form an oblique angle with each other.

A large fold can be seen in the western part of Arsuk Island... This fold is older than that which is associated with the synclinal structure. 
What can be designated as the synclinal structure is not the internal structure of the complex but indicates its boundary towards the metamorphic areas. »

\section{Les éléments tectoniques analysés}

Dans notre étude, nous avons appliqué les méthodes innovées par notre maître (WEGMANN, 1929) et développées dans son enseignement à l'Institut de Géologie de Neuchâtel, Suisse. Il était tout d'abord nécessaire de reconstruire sur la carte la forme géométrique des structures observées sur le terrain. Pour saisir les variations de ces structures d'un endroit à l'autre, nous avons pris de nombreuses mesures d'orientation de plusieurs éléments tectoniques planaires et linéaires:

Les plans de stratification, de schistosité (de flux ou de fracture. Fourmarier, 1949), de clivage (p. 13) et de diaclase.

Les axes de plis sont définis par leur direction et leur plongement axial rapportés aux coordonnées de symétrie de la roche: axe $b$ perpendiculaire au plan de symétrie ac de la roche. L'ordre de grandeur des plis observables varie entre quelques millimètres et une centaine de mètres. L'orientation de l'axe des grands plis, non mesurable directement sur le terrain, a été déterminée à partir de la projection stéréographique équiangle (hémisphère supérieur). Les intersections analysées dans la région d'Arsuk sont de plusieurs types: intersections $L_{1}$ entre les plans de schistosité $S_{1}$ et les plans de stratification $S_{0}$; intersections $L_{2}$ entre les plans de schistosité $S_{1}$ et de clivage $S_{2}$.

Les axes de boudinage.

Les stries: la direction des mouvements et le sens relatif des derniers déplacements qui se sont produits le long des plans de diaclase sont souvent indiqués par les stries. On distingue les mouvements de faille (déplacement à composante verticale) et les mouvements de décrochement (déplacement à composante horizontale).

\section{Analyse structurale}

Dans les ordres de grandeur supérieure, les structures de la région d'Arsuk ont été mises en évidence par l'établissement d'une carte géologique (planche 12) sur laquelle figurent plusieurs horizons lithologiques repaires. Le modelé des structures est traduit sur les cartes structurales par les "lignes structurales». Suivant le problème analysé, ces lignes correspondent à la direction des plans de couche, de schistosité ou de clivage.

Le territoire étudié à été divisé en un certain nombre de secteurs dans lesquels le style des structures a été chaque fois analysé. Les grands 
secteurs, à géométrie complexe, ont été subdivisés en sous-secteurs d'étendue de plus en plus petite, dans le but d'isoler les structures monoaxiales et de permettre l'étude des tectoniques superposées (structures métachrones) et des interférences (structures synchrones; WegmanN, 1947, 1948 et 1959; Wegmann \& Schaer, 1962).

L'étude des plis (d'amplitude allant du centimètre au décamètre), de la schistosité et du clivage, ainsi que les résultats obtenus à l'aide de levés très détaillés (à l'échelle du 1:500 et 1:5000) ont permis de préciser la chronologie assez complexe des déformations.

Les observations macroscopiques ont été complétées en laboratoire par un examen microscopique d'une quinzaine d'échantillons orientés, prélevés principalement dans les charnières et dans les flancs des plis bien individualisés. Il est évident qu'un nombre plus important d'échantillons orientés aurait permis de tirer davantage de renseignements et de serrer la chronologie dont quelques points restent encore obscurs.

\section{Chronologie des déformations}

Trois directions principales de plis superposés ont été définies. Un système plus ou moins $\mathrm{N}-\mathrm{S}$, contemporain du développement de la schistosité, est déformé (ainsi que la schistosité) par un système de plis sensiblement $\mathrm{E}-\mathrm{W}$ accompagnés d'un clivage de crénulation. Le clivage est à son tour déformé par des plis N-S.

Cette superposition de structures métachrones a été mise en évidence dans trois secteurs: sur Arsuk $\emptyset$, à Ørnevæggen et Isua ( $1^{\text {re }}$ et $2^{\mathrm{e}}$ phase) et à Taylers Havn (3 phases).

\section{Les déformations kétilidiennes}

\section{La première phase de déformation: plis contemporains de la schistosité}

Les déformations les plus anciennes que nous ayons reconnues dans la région d'Arsuk $\varnothing$ correspondent au développement de la schistosité. Dans les roches du groupe volcanique, les déformations dues à la schistosité sont mises en évidence par:

L'aplatissement et l'allongement parfois considérable des pillows. Ceux-ci ayant génétiquement des formes et des dimensions variables, on ne dispose d'aucune unité de référence pour mesurer l'aplatissement et l'allongement relatif.

L'aplatissement et l'allongement des fragments de lapillis.

Dans certains pillows, on observe une déformation des fentes de retrait. Celles qui sont en "zone " avec les plans de schistosité sont tordues dans la bordure du pillow. 
Les filons discordants à amphiboles brunes, d'âge kétilidien, sont découpés par les plans de schistosité en tranches parallèles, légèrement décalées les unes par rapport aux autres. Le déplacement peut être mesuré.

L'aplatissement et l'allongement des sphérolites qui caractérisent la bordure des pillows. Le degré d'aplatissement et la direction de l'allongement de ces sphérolites peuvent être mesurés, comme dans le cas des oolites (CLoos, 1946).

Les plis contemporains de la schistosité dans le groupe volcanique

Le développement de la schistosité est accompagné de la formation de plis. L'un d'entre eux est visible dans la paroi d'Ørnevæggen, dans la région Nord-Ouest d'Arsuk Ø (fig. 32).

Ce pli, déjeté vers l'Ouest, affecte surtout l'unité moyenne du Groupe d'Arsuk. En s'écartant des contacts inférieur et supérieur de cette unité, le pli est de moins en moins marqué et à une centaine de mètres des contacts, il n'apparait plus.

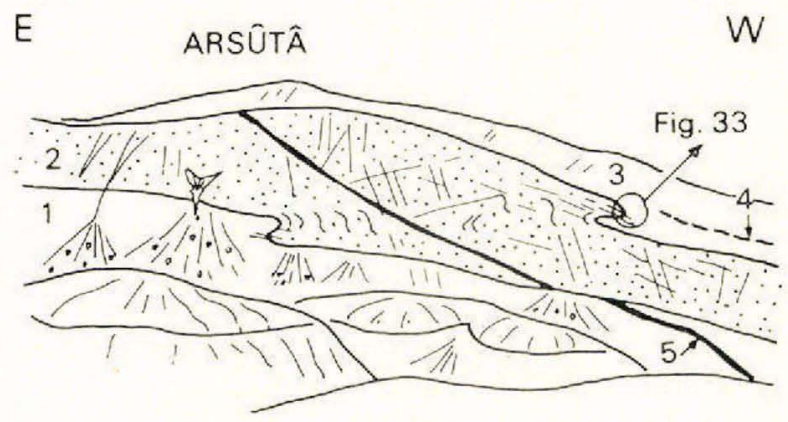

Fig. 32. Pli couché vers l'Ouest, contemporain de la schistosité (1 ${ }^{\text {re }}$ phase), observé dans la falaise Ørnevæggen depuis le point $210 \mathrm{~m}$, côte Nord d'Arsuk $\varnothing$.

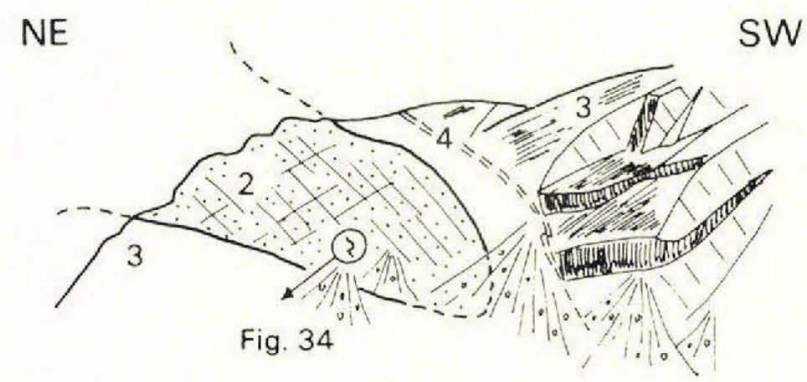

Fig. 33. Détail de la charnière supérieure du pli d'Ørnevæggen. 1: unité inférieure. 2: unité moyenne. 3: unité supérieure. 4: tuf́s. 5: filon basique Gardar. 


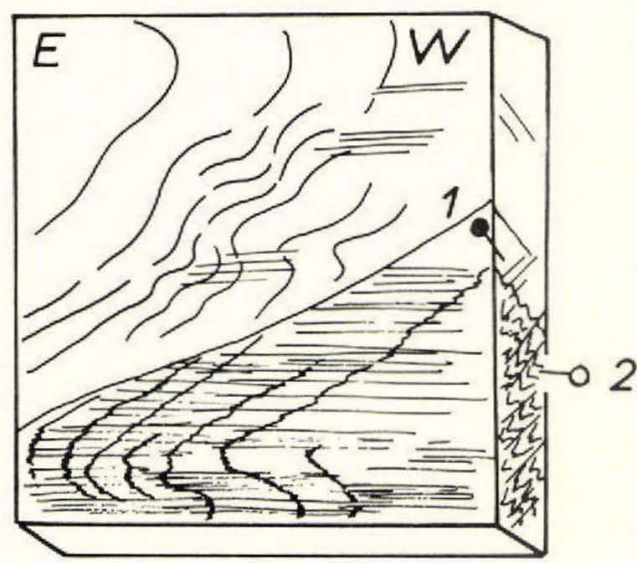

Fig. 34. Détail de la charnière du pli d'Ørnevæggen. Pli de cisaillement. La schistosité est parallèle au plan axial du pli. Les plis (1) et la schistosité sont déformés par les plis (2) accompagnés de clivage ( $2^{e}$ phase).

Les conditions d'affleurement et la forte déformation des pillows n'ont pas permis de construire les axes de ce grand pli à l'aide de la projection stéréographique. Néanmoins, sa direction et son plongement axial ont été évalués grâce à la présence de petits plis d'une dizaine de centimètres d'amplitude mesurés dans la charnière supérieure du grand pli (fig. 33). Ces petits plis ont une direction N-S à NE-SW et plongent vers le Sud

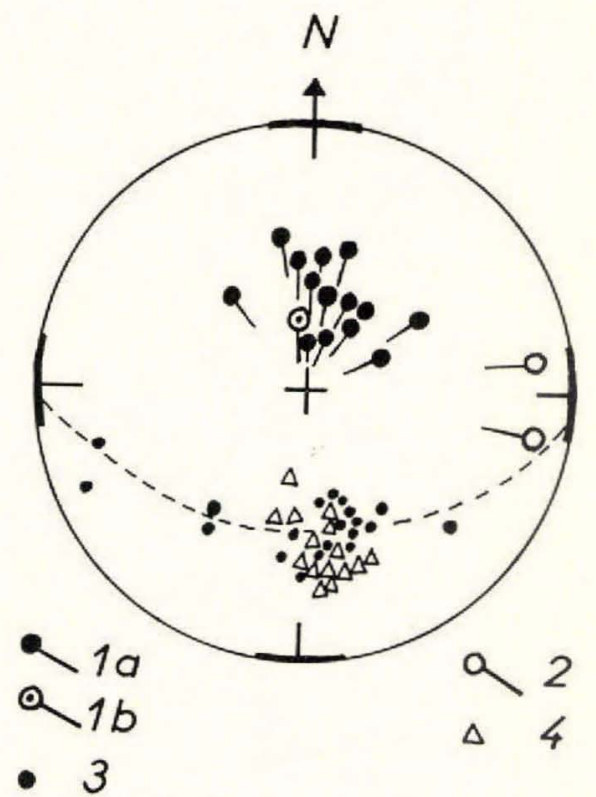

Fig. 35. Stéréogramme des plis de la charnière d'Ørnevæggen. Axes de plis mesurés (1 a) et axe de pli construit (1 b), contemporains de la schistosité. Axes de plis mesurés (2) de la $2^{\mathrm{e}}$ phase. Stratification (3) et schistosité (4). 


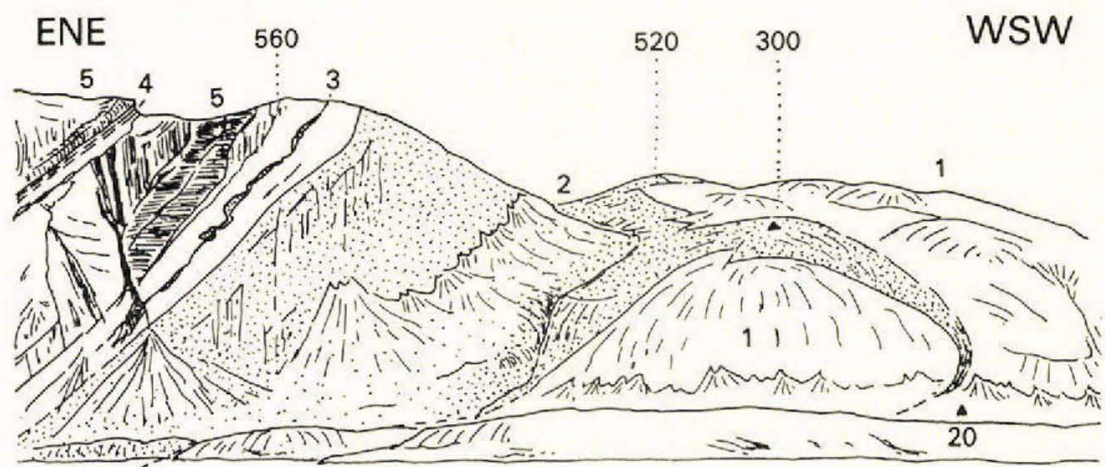

UMINǴMAIT

$a$

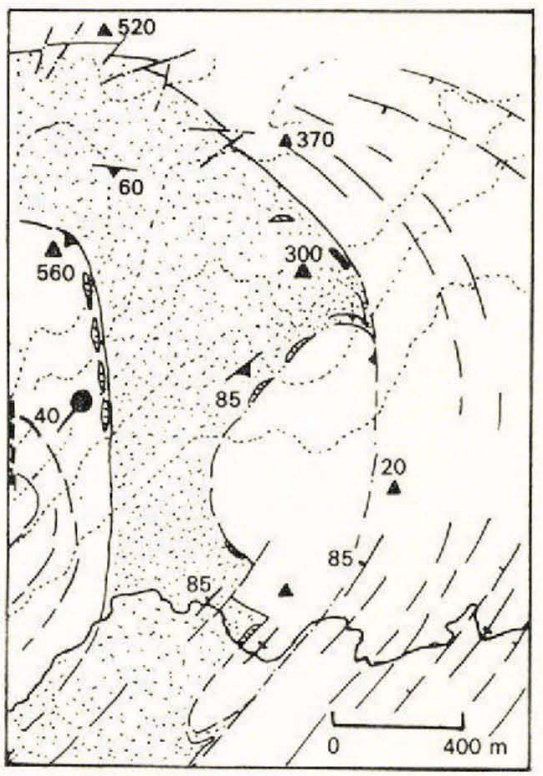

$b$

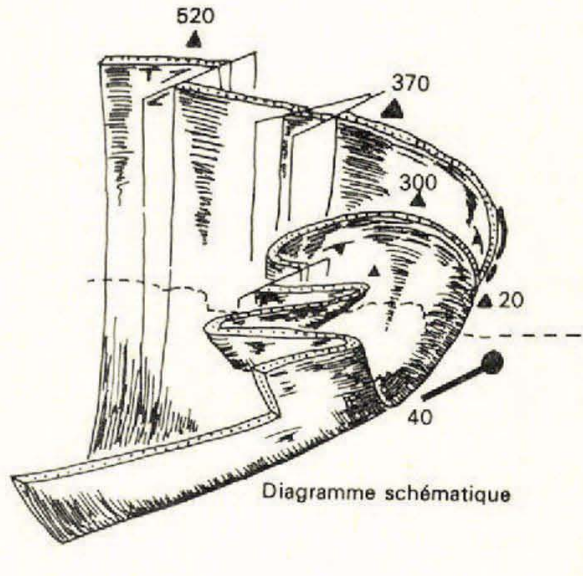

$c$

Fig. 36 .

a. Coupe lithologique observée dans la falaise Sud-Est de Lillesøster Fjeld. 1: pillows de l'unité inférieure. 2: pillows de l'unité moyenne. 3: lentilles quartzitiques. 4: talcschistes. 5: pillows de l'unité supérieure.

b. Les structures de la $1^{\text {re }}$ et de la $2^{\mathrm{e}}$ phase dans le secteur d'Umingmait. Une charnière de pli contemporain de la schistosité est visible au Sud du point $300 \mathrm{~m}$. Cette structure est repliée par le synclinal de direction NE et plongeant au SW qui affecte l'ensemble du groupe volcanique.

c. Diagramme schématique représentant la superposition des structures dans les secteurs d'Umingmait-Lillesøster Fjeld. 
de $40^{\circ}$ à $60^{\circ}$. Ils ont tendance à se déjeter vers l'Ouest. Dans ce même secteur, d'autres plis, de 1 à $2 \mathrm{~m}$ d'amplitude, ont une direction semblable. Ils déforment des bancs de tufs et de schistes noirs à la base de l'unité supérieure.

La schistosité est parallèle au plan axial des petits plis, comme on peut le voir sur la figure 34. Cette schistosité est localement déformée par des petits plis de direction sensiblement $\mathrm{E}-\mathrm{W}$, plongeant vers l'Ouest d'une dizaine de degrés et accompagnés d'un clivage de crénulation. La dispersion des axes de plis de la 1 re phase, comme on le voit sur le stéréogramme de la fig. 35, peut provenir de la superposition de ces deux déformations.

Dans la région Sud-Est d'Arsuk $\varnothing$ (au Nord d'Umingmait, au SudEst du point 300) se trouve un grand pli déjeté vers l'Ouest, du même type que celui de la paroi d'Ørnevæggen. La charnière inférieure de ce pli est très pincée (fig. 36).

Les structures contemporaines de la schistosité dans le groupe sédimentaire

L'étude détaillée du secteur d'Isua a permis de mettre en évidence des structures appartenant à la $1^{\text {re }}$ phase de déformation. On distingue:

des plis de cisaillement et des "intersections 》

des veines de quartz plissées et des "rods".

Ces structures sont en général très déformées par des plis appartenant à la $2^{\mathrm{e}}$ phase de déformation.

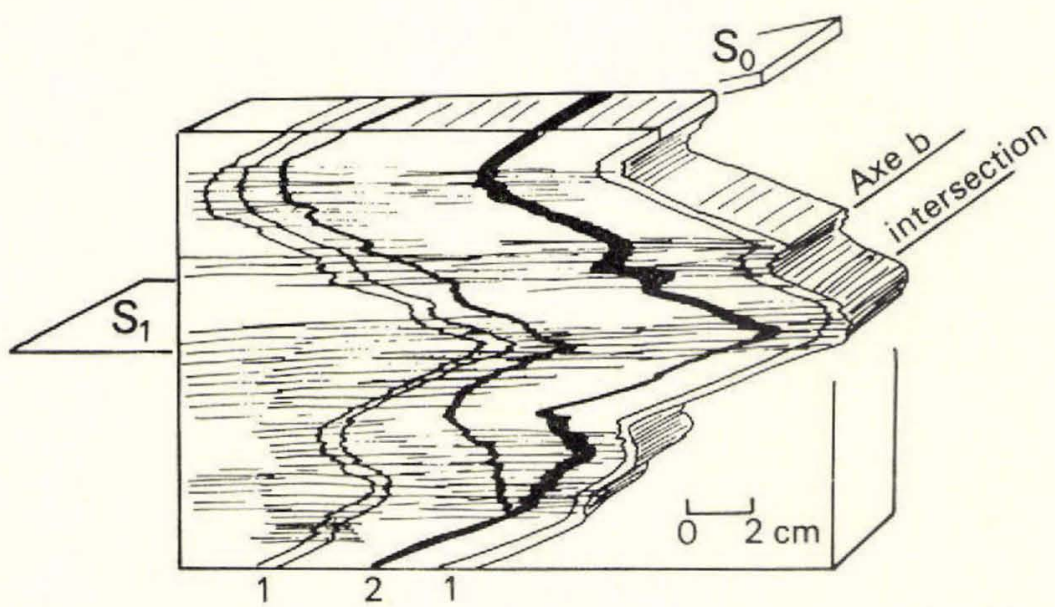

Fig. 37. Diagramme schématique montrant les relations existant entre la schistosité, la stratification, les intersections et les axes b des plis de cisaillement de la $1^{\text {re }}$ phase. $S_{0}$ : stratification, $S_{1}$ : schistosité. 1 : lits quartzitiques dans les phyllites de la Formation d'Isua. 2: veinules de quartz subconcordantes. 


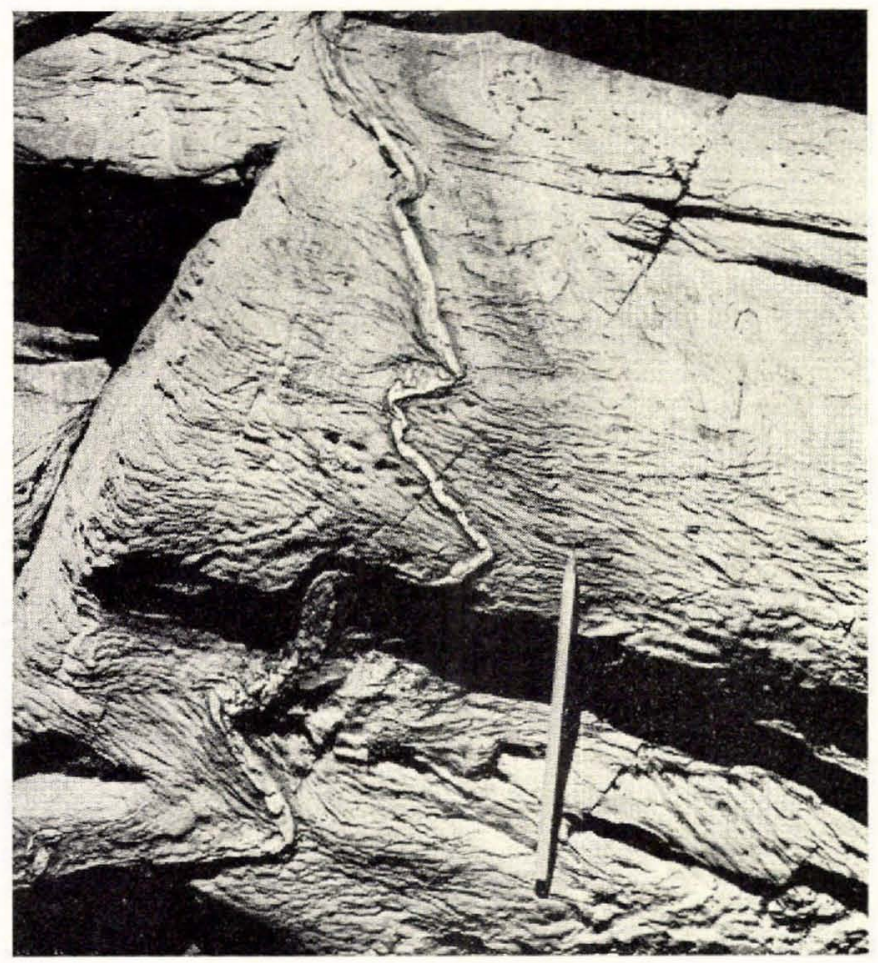

Fig. 38. Phyllites rubanés et veinule de quartz subconcordante, déformés par des plis de la $1^{\text {re }}$ phase de direction $042^{\circ}$, plongeant de $30^{\circ}$ vers le SW. Le rubanement correspond ici à la stratification. Isua.

\section{Les plis de cisaillement et les «intersections 》}

Les plis contemporains de la schistosité se rattachent par définition aux plis de cisaillement. Les plans de schistosité sont parallèles au plan axial des plis. Ils jouent le rôle de plan de mouvement (glissement) lors de la formation des plis. Bien que les déplacements soient infimes, ils sont suffisants pour produire des décalages dans les lits de composition lithologique différente, comme l'ont montré Turner \& Weiss dans le cas des "slip or shear fold" (1963). Ces décalages se traduisent par la formation de minuscules "bourrelets", allongés parallèlement aux axes $b$ des plis de cisaillement et que nous avons désignés sous le nom d'intersections (intersection entre la schistosité et la stratification; fig. 37).

\section{Les veines de quartz plissées et les «rods »}

Dans les sédiments pélitiques, on observe fréquemment de minces veines de quartz, de 1 à $5 \mathrm{~cm}$ d'épaisseur, subconcordantes avec la stratification et déformées, comme les sédiments, par les plis de cisaillement (fig. 38). Ces structures correspondent aux "plis minuscules» de FourMARIER (1951). 


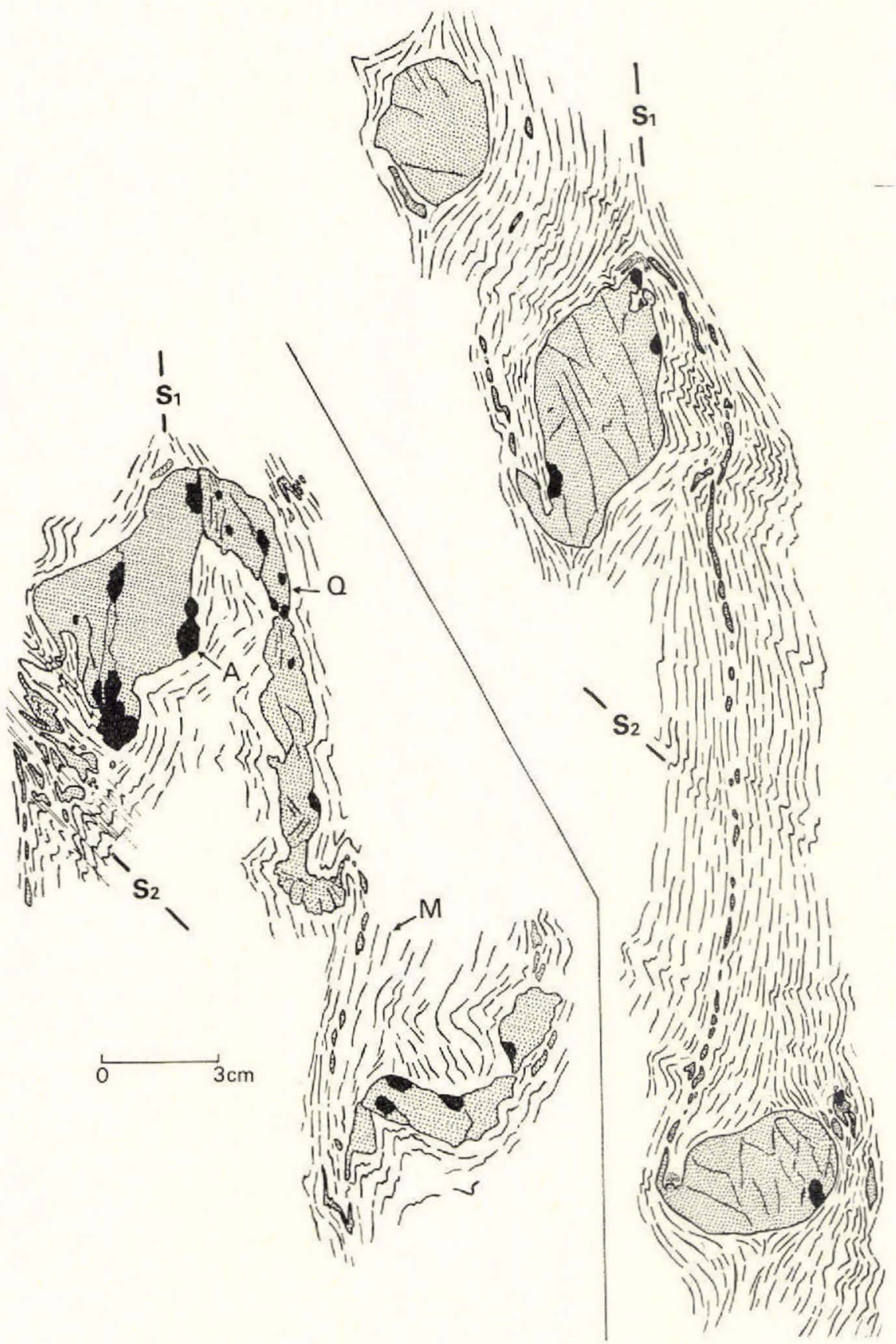

Fig. 39. Structures en rods dans les phyllites de la Formation d'Isua, à Isua. Veines de quartz (Q) avec un peu d'albite (A) plissées lors de la $1^{\text {re }}$ phase. La muscovite (M), disposée parallèlement à la schistosité $\left(S_{1}\right)$ est déformée ainsi que les veines de quartz par la $2^{\mathrm{e}}$ phase accompagnée d'un clivage très peu marqué. 
Certaines veines quartzofeldspathiques plissées sont caractérisées par de nombreux renflements correspondant à des «rods» (G. WiLson, 1961). La schistosité, parallèle au plan axial des plis des veines quartzofeldspathiques, est déformée par de très petits plis en "accordéon 》 (fig. 39). Ces petits plis, dont les axes de direction E-W plongent vers l'Ouest de quelques degrés, appartiennent à la $2^{\mathrm{e}}$ phase de déformation.

\section{La deuxième phase de déformation: plis déformant la schistosité}

Dans la région d'Arsuk, la distinction entre les tectoniques superposées et les interférences a été établie grâce à la schistosité d'après le principe suivant: un élément tectonique est ancien s'il est repris par une nouvelle déformation.

La formation des plis de la $1^{\text {re }}$ phase a engendré une schistosité. Celle-ci est parallèle au plan axial des plis. La schistosité ainsi que les axes de plis et les intersections de la 1 re phase ont été déformés par de nouveaux plis accompagnés d'un clivage de crénulation plus ou moins bien marqué (p.13 et planche 4a) qui appartiennent à la $2^{\mathrm{e}}$ phase de plissement. Cette superposition de structures métachrones est illustrée sur la fig. 40 (voir également planche 4 b).
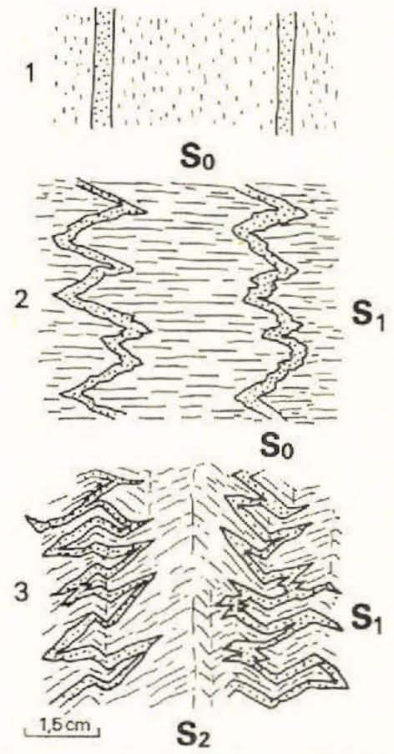

Fig. 40. Déformations successives des phyllites rubanés de la Formation d'Isua, à Isua. - La stratification $S_{0}$ est marquée par l'alternance des lits quartzitiques et phylliteux. - Première phase de plissement: formation des plis contemporains de la schistosité. La schistosité $S_{1}$ est parallèle au plan axial des plis. - Deuxième phase de plissement: déformation des plis précédants et de la schistosité par des plis $080^{\circ}$, plongeant de $30^{\circ}$ vers l'W, accompagnés de clivage $S_{2}$. Cette superposition de structures métachrones est illustrée par la planche $4 \mathrm{~b}$. 
Le style des plis liés à la $2^{\mathrm{e}}$ phase de plissement varie suivant la nature lithologique du matériel soumis aux déformations. Structuralement, en peut diviser les roches de la série supracrustale d'Arsuk $\varnothing$ en deux complexes tectoniques qui sont:

\section{un complexe tectonique} supérieur comprenant un complexe tectonique inférieur comprenant l'unité supérieure

l'unité moyenne l'unité inférieure

l'unité de transition du groupe volcanique le groupe sédimentaire

\author{
du groupe \\ volcanique
}

La planche 13 met en évidence la différence des structures existant entre ces deux complexes séparés par une zone de disharmonie. Celle-ci se situe approximativement au niveau de l'horizon des phyllites pyriteux, avec cherts rubanés, intercalé entre l'unité de transition et l'unité inférieure du groupe volcanique. Lors de la $2^{\mathrm{e}}$ phase de déformation, le complexe supérieur a été plissé en forme de synclinal d'orientation générale NE à ENE, très légèrement déversé vers le Nord. Dans le complexe inférieur, on note la présence de plis serrés plus ou moins couchés au Nord et accompagnés de chevauchements.

\section{Les structures dans le complexe tectonique inférieur}

\section{La côte orientale d'Arsuk $\emptyset$}

Dans la falaise de Skælsiden, on observe une série d'écailles inclinées vers le Sud et des plis de 10 à $50 \mathrm{~m}$ d'amplitude, de direction E-W (fig. 41). Dans la partie inférieure de la falaise, ces plis sont parfois déjetés vers le Sud; dans la partie supérieure de la falaise, ils ont tendance à se déjeter vers le Nord. Ces plis sont recoupés par des surfaces de chevauchement qui deviennent plus fréquentes lorsqu' on se rapproche du contact avec les roches de l'édifice volcanique.

Les gros filons-couches de roches vertes intercalés dans les sédiments ont provoqué de nombreuses disharmonies. A leur contact, les phyllites pyriteux ou rubanés de la Formation d'Isua ont été déformés par des plis d'entraînement dont les axes, plus ou moins horizontaux, ont une direction E-W (planche 13). Les filons-couches ont été laminés et divisés en gigantesques amygdales ou écailles "emballées" dans les phyllites; ces amygdales se sont déplacées les unes par rapport aux autres et se sont chevauchées. Les "télescopages" de ces masses rigides sont fréquents (Cuoos, 1961). Ils sont accompagnés d'une mylonitisation des roches vertes avec "injection" des phyllites dans les zones broyées. Les mouvements sont très complexes; certains plis d'entraînement $\mathrm{E}-\mathrm{W}$ montrent que les compartiments supérieurs se sont déplacés relativement vers le 


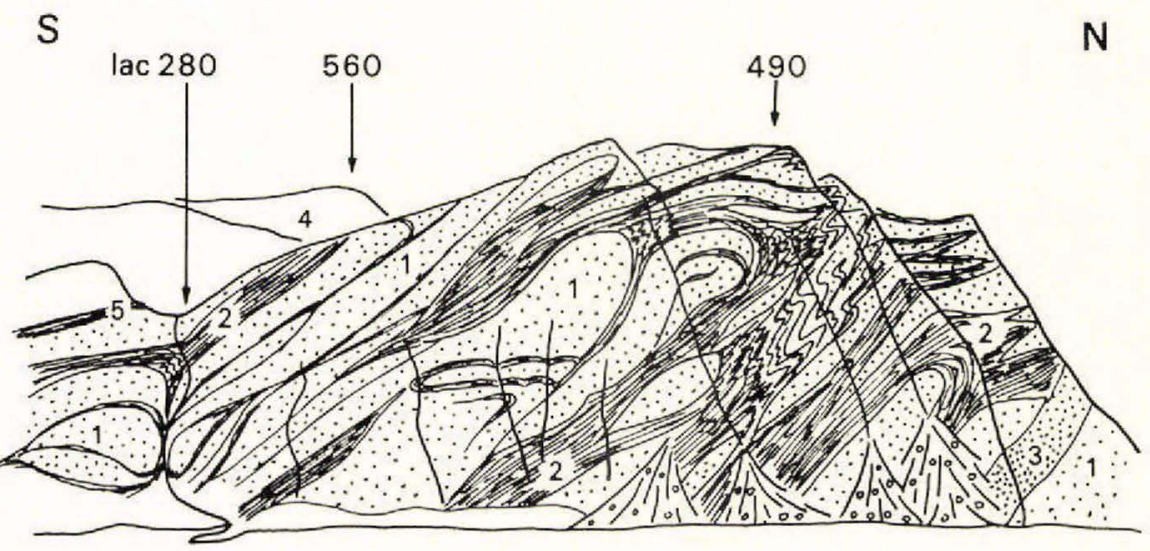

Fig. 41. Structures de la falaise de Skælsiden, côte Est d'Arsuk Ø. Les écailles de roches vertes (1), emballées dans les phyllites et les schistes actinolitiques de la formation d'Isua (2), ont glissé les unes sur les autres en provoquant de nombreuses disharmonies. Ces écailles sont constituées par des filons-couches intercalés dans les sédiments et par les roches vertes de l'unité de transition du groupe d'Arsuk. 3: filon volcanique Gardar. 4: pillows de l'unité inférieure. 5: horizon de phyllites pyriteux avec chert du groupe volcanique.

Nord. D'autres plis indiquent des mouvements inverses. On note encore des mouvements ayant donné naissance à des plis d'axes N-S.

L'horizon des phyllites pyriteux avec cherts lenticulaires, qui se trouve au sommet de la Formation d'Isua, a subi des déformations intenses. Il constituait, en effet, un "coussin " sur lequel ont navigué les écailles de l'unité volcanique de transition. Au Nord d'Eqalugârssuit, cet horizon se réduit à quelques lambeaux disposés le long des plans de mouvement. Un second "coussin » est représenté par l'horizon des phyllites pyriteux avec « quartzitic slabs » situé entre l'unité de transition et l'unité inférieure du groupe voleanique.

Au Sud de Skælsiden, entre Eqalugârssuit et Nákarneq, les plis $\mathrm{E}-\mathrm{W}$ sont régulièrement déjetés vers le Nord (planche 15). Ils appartiennent au flanc Nord du grand synclinal d'Arsuk $\varnothing$. Ces plis se relient au Sud à une structure de type anticlinal dont le coeur est occupé par les gneiss et migmatites des îles Napassut, Narssap sagdlia et Tavdlorutit. Le repli synforme de l'îlot Evqitsut appartient probablement au flane Nord de cette structure anticlinale méridionale, d'orientation NE ou ENE, plongeant vers l'Ouest de $30^{\circ}$ à $50^{\circ}$.

\section{Le versant Nord d'Arsuk $\emptyset$}

Les structures en plis couchés et en écailles de Skælsiden sont recoupées, presque parallèlement à leur axe, par le versant Nord d'Arsuk $\varnothing$. L'ensemble de ces structures, de direction $\mathrm{E}-\mathrm{W}$, plonge sensiblement vers 
l'Ouest. Ce plongement est perturbé localement par des plis $\mathrm{N}-\mathrm{S}$, déformant également la schistosité. La chronologie entre ces deux systèmes de plis n'a pas été établie.

Entre Niaqornarssuaq et Blålershavn, la Formation d'Isua vient en contact avec celle de Taylers Havn par l'intermédiaire d'une surface de chevauchement qui coincide, dans ce secteur, avec l'horizon des schistes pyriteux et graphiteux.

\section{Le secteur d'Ikerasârssuk Nord (au Nord d'Arsuk Ø)}

$\mathrm{Au}$ Nord du détroit d'Ikerasârssuk, les banes de quartzites massifs ont une orientation sensiblement $\mathrm{E}-\mathrm{W}$ et un pendage de $50^{\circ}$ à $60^{\circ}$ vers le Sud. Les quartzites dolomitiques qui les surmontent sont déformés principalement par des plis ENE-WSW, empilés les uns sur les autres. Certains plis ont nettement tendance à se déjeter vers le Sud (fig. 9). Les plis disharmoniques entre les différents bancs quartzitiques, séparés par des niveaux carbonatés, sont spectaculaires (fig. 10).

Cette structure avec plis déjetés vers le Sud pourrait correspondre au flanc inverse d'un synclinal faillé couché au Nord. Ce style correspondrait assez bien avec celui observé dans la falaise de Skælsiden, au Sud du détroit d'Ikerasârssuk. Le flanc Nord de ce synclinal n'apparaît pas. Il peut être recoupé par une faille car les quartzites dolomitiques sont séparés des quartzites massifs par une bande herbeuse de quelques mètres de largeur. Les nombreux replis des quartzites dolomitiques correspondraient à des plis d'entraînement liés à la structure synclinale majeure.

La variation de plongement des axes E-W sur le stéréogramme 18 de la planche 13 est liée, dans une certaine mesure, aux disharmonies observées de couche à couche dans les quartzites dolomitiques. Elle est due également à la présence de petits plis N-S. La chronologie entre ces deux systèmes de plis n'a pas été établie à cet endroit.

\section{Le secteur d'Inugsukasik (côte Nord-Ouest d'Arsuk Ø)}

A Inugsukasik, dans les phyllites rubanés de la Formation d'Isua, la schistosité contemporaine de la 1 re phase est déformée par des plis d'orientation variée dont le style se rapproche beaucoup de celui des plis de flux. Les figures de déformation évoquent une grande mobilité des roches (fig. 14). Les plis, de direction $\mathrm{E}-\mathrm{W}$ montrent une variation considérable du plongement axial (stéréogrammes 15 et 16, planche 13). Par exemple, un pli de direction NNE-SSW, de $50 \mathrm{~cm}$ d'amplitude, passe d'un plongement de $40^{\circ}$ vers le Sud à un plongement vertical sur une distance de $1 \mathrm{~m}$.

Les roches basiques intercalées dans les sédiments sont également plissées. Dans la partie centrale des filons-couches, les roches restent assez massives et sont affectées de multiples déchirures et fractures ouvertes. 
Au contact avec les sédiments, elles sont très schistosées et les axes des plis qui les déforment sont parallèles à ceux mesurés dans les phyllites.

\section{Superposition des structures de la $1^{\text {re }}$ et de la $2^{\mathrm{e}}$ phase à Isua}

Sur la côte orientale d'Arsuk $\varnothing$, au Nord de la pointe d'Isua, une carte levée à l'échelle du 1:500 dans les phyllites rubanés de la Formation d'Isua révèle l'existence de deux systèmes de plis superposés (planche 15).

Les structures les plus apparentes consistent en une succession de synformes et antiformes de 20 à $30 \mathrm{~m}$ d'amplitude, légèrement couchés vers le Nord, d'orientation sensiblement $\mathrm{E}-\mathrm{W}$ et plongeant vers l'Ouest de $20^{\circ}$ à $40^{\circ}$ en moyenne. Ces plis, qui sont localement accompagnés d'un clivage de crénulation, appartiennent à la $2^{\mathrm{e}}$ phase de plissement qui déforme la schistosité. Des plis de cisaillement et des intersections appartenant à une phase plus ancienne sont visibles dans les flancs des plis de la $2^{\mathrm{e}}$ phase. IIs sont déformés et pliés dans les charnières de ces derniers. La fig. 42 met en évidence les faits suivants:

les axes de plis qui déforment la schistosité sont tous orientés dans une direction sensiblement E-W (fig. $42 \mathrm{~b}$ ); les axes de plis et les intersections appartenant au plissement contemporain de la schistosité sont désorientés (fig. 42 a).

Afin de connaître la direction primitive des axes de plis contemporains de la schistosité, nous avons divisé le secteur d'Isua en plusieurs soussecteurs dont les mesures ont été reportées sur les stéréogrammes 1 à 7 de la planche 15. Les stéréogrammes 4 à 7 montrent que les axes de plis et intersections appartenant à la $1^{\text {re }}$ phase de déformation kétilidienne "s'enroulent" autour des axes de plis appartenant à la $2^{\mathrm{e}}$ phase.

Théoriquement, la direction des anciens plis peut être déduite par construction géométrique: rabattement sur le plan horizontal de l'axe de pli autour duquel s'enroule la structure repliée, puis déroulement de cette dernière. Si l'axe de rotation correspond à un pli de flexure, les structures sont déroulables et dans ce cas, l'angle entre la structure repliée et l'axe de rotation doit être constant.

L'observation montre cependant que l'angle entre les axes de la 1 re phase (plis et "intersections») et les axes de la $2^{\mathrm{e}}$ phase (axe de plis jouant le rôle d'axe de rotation) varient sensiblement dans chaque secteur considéré.

Stéréogrammes 1 et 2 ; angle variant entre $30^{\circ}$ et $50^{\circ}$

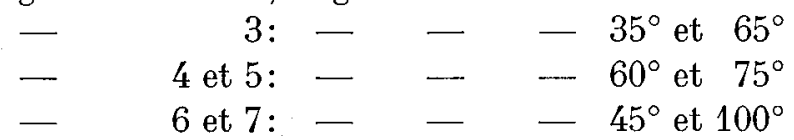




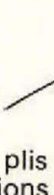

70 axes de plis et intersections
de la 1re phase
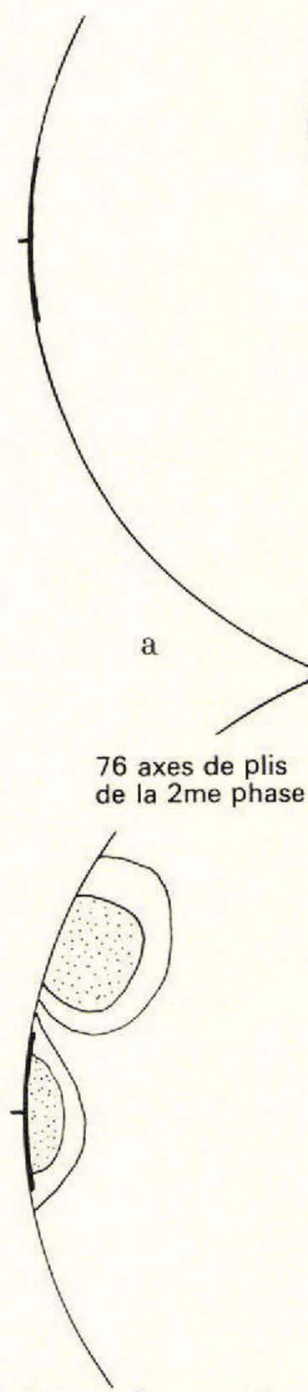

HÉMISPHËRE SUPÉRIEUR
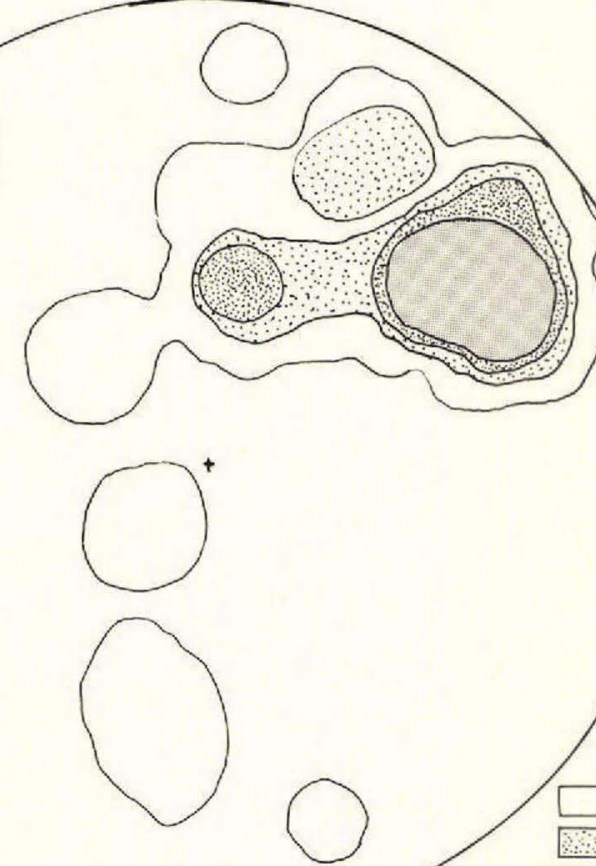

$2-6 \%$

$7-9 \%$ 30-11\% $12-15 \%$ $16-19 \%$ de la 2 me phase

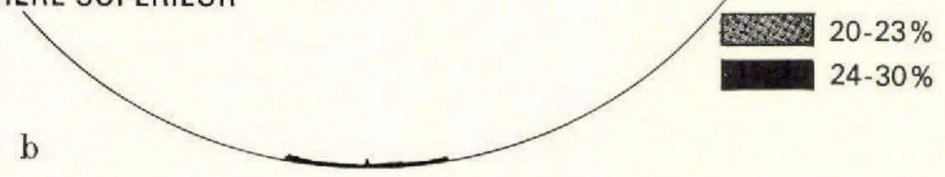

Fig. 42. Stéréogrammes (équisurfaces) des axes de plis de la $1^{\text {re }}$ phase (a) et de la $2^{\mathrm{e}}$ phase (b). 
Cette variation s'explique en partie par la disposition légèrement en éventail des axes de plis de la $2^{\mathrm{e}}$ phase qui font fréquemment entre eux un angle de $20^{\circ}$ à $30^{\circ}$ (planche 15 et fig. $42 \mathrm{~b}$ ). La même disposition devait probablement caractériser déjà les axes de plis de la $1^{\text {re }}$ phase avant leur déformation.

La construction des lieux géométriques des structures repliées permet de définir des valeurs angulaires moyennes (stéréogrammes 4 à 7 de la planche 15). Après rabattement de l'axe de rotation sur le plan horizontal, on peut connaître la direction primitive des plis de la 1 re phase. Celle-ci varie entre $045^{\circ}$ et $065^{\circ}$, avec des valeurs allant même jusqu'à $005^{\circ}$, comme sur les stéréogrammes 6 et 7 .

La structure synclinale ou en "baignoire" du complexe tectonique supérieur

La structure du complexe tectonique supérieur est caractérisée par un grand synclinal d'orientation NE à ENE dont la charnière n'est visible que dans la région Sud-Est d'Arsuk $\varnothing$. La direction et le plongement de ce synclinal varient $d u$ NE au SW, c'est-à-dire lorsqu'on monte dans la série lithostratigraphique.

Dans l'unité lithostratigraphique inférieure, la charnière synclinale est largement évasée en direction du NE. Son axe plonge vers le SW de $40^{\circ}$ (stéréogramme 11, planche 13). Ce pli synclinal semble correspondre à un pli de flexure. Les pillows sont peu déformés dans la charnière. Dans l'unité moyenne, l'axe du synclinal plonge de $40^{\circ}$ à $45^{\circ}$ vers le SW (stéréogramme 12). La schistosité qui affecte les pillows de cette unité et qui est contemporaine de la 1 re phase, est repliée dans la charnière synclinale (stéréogramme 12a).

Dans l'unité supérieure, la charnière synclinale est très pincée. Elle est visible dans le secteur de Lillesøster Fjeld. Les pillows y sont en général écrasés. L'axe plonge de $60^{\circ}$ à $65^{\circ}$ vers le $\mathrm{SW}$ (stéréogramme 10). En allant vers l'Ouest, le plongement diminue jusqu'à $45^{\circ}$ et même $35^{\circ}$ (stéréogrammes 8 et 9 ).

Comme on le voit, ce pli synclinal n'est pas cylindrique. La charnière du synclinal est caractérisée par une disposition en éventail des axes de pli ou de courbure. Ce type de structure est comparable à la partie évasée d'une baignoire.

Le flanc Sud du synclinal n'est visible que sur une très petite partie de la côte Sud-Est d'Arsuk $\varnothing$. Il est vertical à Umingmait et se renverse légèrement sur le flanc Sud de Lillesøster Fjeld.

A l'Ouest de Lillesøster Fjeld, l'axe du synclinal n'a pu être construit qu'à partir de son flanc Nord, car la charnière du pli disparaît dans la 
$\mathrm{E}$

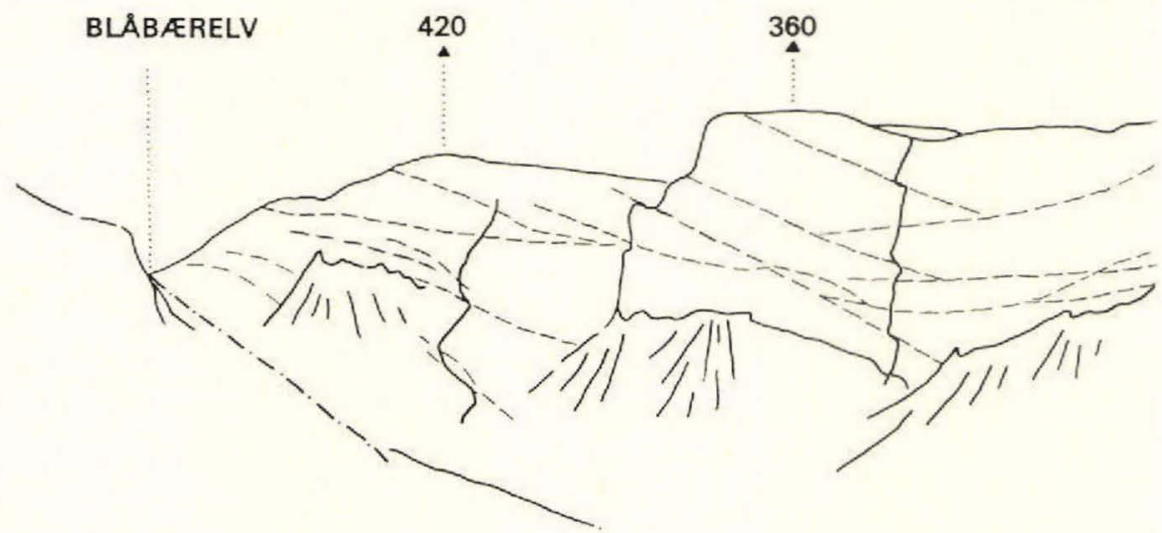

Fig. 43. Zones de cisaillement recoupant les roches vertes de l'unité volcanique inférieure. Falaise Sud du ravin de Blåbærelv. Ces zones se relient entre elles et déterminent de grandes amygdales qui ont joué le rôle d'articulations lors des déformations de l'édifice volcanique durant la $2^{\mathrm{e}}$ et la $3^{\mathrm{e}}$ phase kétilidienne.

mer. Dans la moitié occidentale d'Arsuk Ø, le flane Nord a une direction sensiblement ENE-WSW (stéréogrammes 1 à 6 , planche 13). Sur la côte méridionale de l'île, les couches sont verticales. En allant vers le Nord, elles se couchent de plus en plus jusqu'à un pendage Sud de $30^{\circ}$ à $40^{\circ}$. Les couches sont affectées en outre de larges ondulations (de quelque $400 \mathrm{~m}$ d'amplitude), dont l'axe, sensiblement horizontal, est parallèle à celui de la structure principale ENE-WSW. D'autres ondulations leur sont perpendiculaires. Elles sont visibles sur la côte Sud d'Arsuk $\varnothing$ (stéréogrammes 2 et 6).

Lors du plissement de l'énorme masse de matériel volcanique, les anciens plans de discontinuité ont été réutilisés, notamment les plans de schistosité se trouvant dans l'unité volcanique moyenne. Dans la charnière synclinale, les pillows ne sont pratiquement plus reconnaissables. Les mouvements, très marqués dans certaines couches (niveaux de tufs, de cherts, intercalations d'ultrabasites), ont provoqué des disharmonies.

Les roches vertes de la série volcanique ont été recoupées également par de nombreuses zones de cisaillement (shear zone) discordantes, de 0,5 à $50 \mathrm{~m}$ d'épaisseur, qui ont joué le rôle d'articulations, facilitant ainsi la torsion des couches. Les roches y sont débitées en feuillets plus ou moins minces, plissotés et cisaillés. Les axes de plis (Totenfalten) y ont une direction E-W, parfois NE-SW. Ces zones de cisaillement sont caractérisées par leur allure ramifiée, discontinue et leur changement rapide d'épaisseur. Elles se rejoignent parfois en déterminant d'énormes amygdales, de plusieurs dizaines de mètres d'épaisseur (fig. 43). Les plus importantes se trouvent à la base de la formation volcanique. Il est difficile 
de préciser si toutes les zones de cisaillement sont liées à la formation du synclinal d'Arsuk. Les relations chronologiques montrent qu'elles décalent les filons amphibolitiques kétilidiens sur l'îlot au Nord de Hekseø). Elles sont recoupées par les filons lamprophyriques, témoins d'une tectonique cassante d'âge Gardar. Certaines zones de cisaillement ont été réutilisées lors des phases tardives de l'activité orogénique kétilidienne et sans doute aussi pendant la période Gardar. Les feuillets schisteux, très froissés, sont déformés par des plis d'entrainement qui sont parfois roulés sur eux-mêmes (structure en fuseau). On y observe également la formation de brèches tectoniques.

\section{La troisième phase de déformation: plis déformant le clivage}

La $3^{e}$ phase de déformation est caractérisée par la déformation des surfaces de clivage qui sont associées aux plis de la $2^{\mathrm{e}}$ phase. Ce clivage, souvent assez frustre sur Arsuk Ø, est très bien développé dans le secteur de Taylers Havn.

Dans le chapitre Lithostratigraphie, les structures microscopiques des roches du secteur de Taylers Havn ont été décrites en détail et nous n'en rappellerons que les principaux caractères:

La stratification, à l'intérieur des bancs de même lithologie, n'est plus visible. Elle est masquée par les cristallisations et déformations accompagnant le développement de la schistosité et du clivage.

La schistosité est déformée par des petits plis accompagnés d'un clivage de crénulation. Sur les plans de clivage, on observe de fines structures linéaires correspondant à des «intersections " $L_{2}$ entre le clivage $S_{2}$ et la schistosité $S_{1}$. Ces intersections sont parallèles aux axes des petits plis $F_{2}$ déformant la schistosité.

Les plans de clivage sont à leur tour déformés par des plis $F_{3}$ appartenant à la structure synclinale $\mathrm{N}-\mathrm{S}$ de Taylers Havn (planche 3a). Des plaques minces, taillées perpendiculairement aux axes de plis $F_{2}$ et intersections $L_{2}$, sont caractérisées par la présence de plan de clivage $S_{2}$ déformant la schistosité $S_{1}$ qui est plissée (fig. 48). Les plaques minces taillées perpendiculairement aux plis $F_{3}$ montrent que les plans de clivage sont déformés par de petits plis en «accordéon ».

\section{Les structures dans le secteur de Taylers Havn}

Dans la région de Taylers Havn, les structures les plus apparentes correspondent à une série de plis d'orientation N-S à NNE-SSW et plongeant vers le Sud de $40^{\circ}$ à $55^{\circ}$ (planches 16 et 17). Ces plis sont de différents ordres de grandeur. Les niveaux de phyllites et de schistes sont dé- 
formés par des petits plis de 1 à $10 \mathrm{~cm}$ d'amplitude qui se relient pour former des plis de 0,5 à $1 \mathrm{~m}$ d'amplitude; ces derniers constituent des plis plus grands de 5 à $20 \mathrm{~m}$ d'amplitude. Les phyllites intercalés entre les bancs durs (quartzites, etc) sont affectés de plis d'entraînement, de style en "accordéon", de quelques millimètres à $10 \mathrm{~cm}$ d'amplitude. Les niveaux amphiboliques sont boudinés (fig. 8 et 13). Les axes des boudins sont parallèles aux axes de plis décrits plus haut.

Les plus grands plis se relient encore entre eux pour former un synclinal appartenant à une structure plus importante qui se poursuit probablement à l'Est, au-dessus des gneiss de la péninsule d'Ivigtut. Le pli synclinal de Taylers Havn, dont l'amplitude est de quelque $200 \mathrm{~m}$ dans l'horizon des schistes sombres, est beaucoup moins marqué dans l'horizon des quartzites.

Superposition de la $2^{\text {e }}$ et de la $3^{\mathrm{e}}$ phase à Taylers Havn

Dans les schistes sombres rubanés de la côte occidentale de Taylers Havn, au Nord d'Itivsâq, les "intersections " $L_{2}$ et les axes de plis $F_{2}$ font un angle de $33^{\circ}$ à $54^{\circ}$ avec les axes de plis $F_{3}$ appartenant à la structure synclinal N-S de Taylers Havn (fig. 44). Les observations faites dans les quartzites de Ravnenæs confirment ces résultats.

L'orientation primitive des plis de la $2^{\mathrm{e}}$ phase, obtenue après rabattement des axes de rotation sur un plan horizontal, oscille entre $140^{\circ}$ et

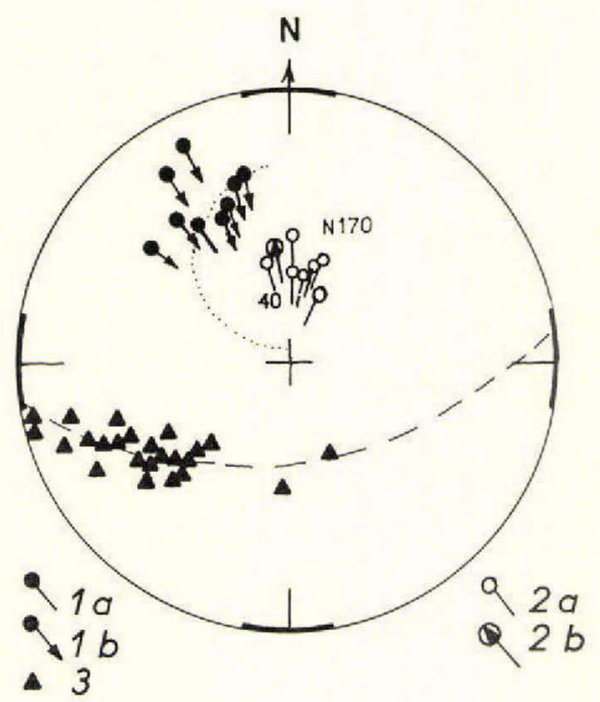

Fig. 44. Stéréogramme des axes de plis de la $2^{\mathrm{e}}$ et de la $3^{\mathrm{e}}$ phase dans les schistes sombres de la côte Ouest de Taylers Havn. Les axes de plis de la $2^{\mathrm{e}}$ phase (1 a) et les intersections $S_{1}-S_{2}(1 \mathrm{~b})$ ainsi que la schistosité et le clivage $S_{2}(3)$ sont déformés par des petits plis (2a) et des grands plis $(2 \mathrm{~b})$ de la $3^{\mathrm{e}}$ phase. 
$165^{\circ}$. Les directions sont assez différentes de celles revelées sur Arsuk Ø:

$140^{\circ}$ à $165^{\circ}$ à Taylers Havn

$080^{\circ}$ à $110^{\circ}$ sur la côte orientale d'Arsuk $\varnothing$.

Il convient de faire quelques remarques sur la méthode de construction utilisée. Le simple rabattement des axes de rotation (plis de la $3^{\mathrm{e}}$ phase) sur le plan horizontal peut introduire des erreurs considérables, notamment dans le cas d'axes très redressés comme ceux de Taylers Havn.

Il n'est pas certain tout d'abord que ces axes de plis aient été initialement horizontaux puis redressés dans le plan vertical.

Lorsque ces plis se sont formés, les couches portant des structures plus anciennes (axe de pli, intersection, etc) ont pu être animées de mouvements de translation et de rotation.

Dans le cas particulier à la région d'Arsuk, il est également possible que tout un complexe tectonique, comme celui constitué par la Formation d'Isua et l'édifice volcanique, ait subi une rotation d'ensemble par rapport au complexe sous-jacent lors des plissements de la $3^{\mathrm{e}}$ phase: rotation par disharmonie se situant par exemple au niveau des horizons graphiteux et pyriteux.

\section{Les plis de la côte Nord d'Arsuk Ø}

Dans les phyllites rubanés du secteur d'Inugsukasik, les plis déformant la schistosité semblent se rattacher aux plis de flux qui sont caractérisés par une grande variabilité d'orientation axiale, liée à la plasticité des roches lors des déformations.

La disposition des axes de plis dans la partie septentrionale d'Arsuk $\varnothing$ (planche 13) met en évidence une variation du plongement des axes de plis $\mathrm{E}-\mathrm{W}$, alors que les plis $\mathrm{N}-\mathrm{S}$ sont régulièrement inclinés au Sud avec un plongement assez constant. Cette observation est valable aussi bien pour les axes de plis mesurés que pour les axes construits qui sont d'un ordre de grandeur supérieur. Si les axes de plis $\mathrm{N}-\mathrm{S}$ avaient été repliés par les axes $\mathrm{E}-\mathrm{W}$, on devrait retrouver, au moins localement, des plis N-S plongeant au Nord, ce qui n'est pas le cas. Par contre, on pourrait expliquer de cette manière les variations notables de plongement des axes E-W perturbés par des nouvelles structures N-S. Des investigations futures devraient permettre de vérifier si ces variations de direction des plis correspondent à des structures synchrones ou métachrones.

\section{La tectonique cassante tardive}

\section{Les diaclases plissées}

Dans le ravin Ivît Elv, à l'altidude $180 \mathrm{~m}$, les pillows sont recoupés par des diaclases ondulées rapprochées et parallèles entre elles qui déter- 
minent des sortes de bancs de 5 à $30 \mathrm{~cm}$ d'épaisseur. Les plis ou ondulations ont un rayon de courbure variant entre $50 \mathrm{~cm}$ et plusieurs mètres. Les axes d'ondulation sont orientés $070^{\circ}$ à $080^{\circ}$ et leur plongement est de $10^{\circ}$ à $60^{\circ}$ vers l'Est.

\section{Les diaclases de cisaillement ENE}

Un système de diaclases d'orientation ENE, inclinées de $40^{\circ}$ à $60^{\circ}$ vers le Nord, se retrouve dans toute la région d'Arsuk $\varnothing$ (planche 14). Dans l'édifice volcanique, ces diaclases sont généralement espacées de 50 à $100 \mathrm{~cm}$, parfois davantage. Dans certains cas, elles sont plus serrées et cisaillent les pillows en produisant un gaufrage de leur croûte. Ces phénomènes de cisaillement accompagnés de "gaufrage par clivage " (Fou rmarier, 1953) sont fréquents dans les roches sédimentaires.

Les diaclases de ce type recoupent toutes les zones de cisaillement kétilidiennes, mais n'affectent pas les filons de lamprophyre. Elles pourraient appartenir à une phase tardive de déformation kétilidienne car elles semblent représenter l'équivalent des zones de cisaillement dans un étage tectonique supérieur.

\section{Les contacts entre les roches supracrustales et les gneiss du socle}

Les contacts entre les roches supracrustales et les gneiss appartenant au vieux socle sont visibles entre Taylers Havn et Webers Havn ainsi qu'au Nord d'Ikerasârssuk.

\section{Les contacts entre Taylers Havn et Taylers Fjeld}

Dans ce secteur, les contacts entre les quartzites kétilidiens et les gneiss prékétilidiens sont tectonisés. La discordance entre les structures des quartzites et celles des gneiss est visible à l'échelle de la carte (planche 16 et 17).

La partie inférieure de l'horizon des quartzites n'existe que dans le secteur de Taylers Fjeld. Au Sud, cet horizon est déformé par un grand pli qui est recoupé par le contact gneiss-quartzite.

$\mathrm{Au}$ Sud de Bjørneelv, le contact entre les quartzites et les gneiss se fait par l'intermédiaire d'une zone bréchique. Ces brèches se sont formées par l'accentuation des plis de cisaillement qui ont découpé les quartzites en petits fragments anguleux. Dans la zone de contact, les plis ne sont plus reconnaissables. Les roches sont très écrasées et les brèches quartzitiques sont cimentées dans une matrice quartzosériciteuse.

Au Nord de Bjørneelv, on observe des phyllonites au contact gneissquartzite et à proximité de celui-ci (fig. 30). Les gneiss sont très écrasés et recoupés par des pegmatites de quartz qui ne traversent pas les quartzites. 
A la pointe de Ravnenæs, le contact est constitué par un plan strié. Les stries sont inclinées de $80^{\circ}$ vers le Sud. A l'extrémité Nord de la baie de Taylers Havn, les gneiss et les quartzites sont déformés par des plis de cisaillement de quelques centimètres d'amplitude qui sont localisés dans la zone de contact.

Enfin à l'altitude $150 \mathrm{~m}$, dans le secteur de Taylers Fjeld, on trouve des écailles de gneiss dans les quartzites et inversement. Les roches sont mylonitisées.

\section{Les contacts au Nord d'Ikerasârssuk et à Webers Havn}

$\mathrm{Au}$ Nord d'Ikerasârssuk, les contacts entre les quartzites et les gneiss sont de nature mylonitique. La mylonite, d'âge Gardar, a une orientation E-W et un pendage de $50^{\circ}$ vers le Sud. Elle est sensiblement parallèle aux bancs de quartzites. Elle se poursuit vers l'Est jusqu'à Webers Havn où elle recoupe transversalement les horizons inférieurs de la Formation de Taylers Havn. Ceux-ci viennent buter contre les gneiss par l'intermédiaire d'une zone broyée de $200 \mathrm{~m}$ d'épaisseur de teinte rougeâtre, inclinée vers le Sud de $50^{\circ}$.

\section{Résumé}

La discordance observée entre les quartzites et les gneiss à Taylers Havn est exclusivement de nature tectonique. Elle est due à un mouvement de réajustement entre le vieux socle et les roches plus jeunes après la phase de plissement qui a donné naissance au synclinal $\mathrm{N}-\mathrm{S}$ de Taylers Havn. Dans la zone de contact, les axes des plis d'entraînement sont presque parallèles à ceux du synclinal de Taylers Havn. Ces plis indiquent un mouvement de translation le long du contact avec déplacement relatif du compartiment kétilidien vers le Nord.

Les mouvements qui suivirent devinrent de plus en plus cassants. On observe des déplacements par faille. D'autres mouvements, plus tardifs encore, contribuèrent à modifier le style de cette discordance et sont caractérisés par des brèches et des mylonites d'âge Gardar.

\section{Les déformations kétilidiennes dans les gneiss du socle}

Dans les gneiss du socle, les différentes phases de déformation kétilidiennes sont difficiles à distinguer des déformations plus anciennes. Elles peuvent être néanmoins décelées en de nombreux endroits grâce au réseau des filons basiques prékétilidiens (?) et kétilidiens.

L'intensité des déformations kétilidiennes croît du Nord au Sud.

Dans la région des îles Tôrnârssuk et Sermersût, ces mouvements ont donné naissance à des zones de cisaillement (shear zone) recoupant 
les gneiss et affectant les filons amphibolitiques discordants (BoNDESEN \& Henriksen, 1965; Weidmann, 1964).

Dans la partie centrale de Grænseland, au Nord du glacier d'Arsuk Bræ, le socle a été recoupé par des failles qui sont parallèles au plan axial des plis de la $2^{\mathrm{e}}$ phase.

Dans les gneiss de la péninsule d'Ivigtut, on observe la superposition de plis NNW-SSE et de plis ENE-WSW (planches 12, 13 et 17). L'examen des structures à l'échelle régionale indique une déformation des plis NNW-SSE par des plis ENE-WSW. Cette chronologie est identique à celle observée dans les gneiss des îles Tôrnârssuk et Sermersût (BerthelSEN, 1960, fig. 1 et 2; Berthelsen et al., 1962, fig. 7 et 8). A première vue, on pourrait croire que les plis ENE sont dus à la $2^{\mathrm{e}}$ phase kétilidienne qui a sensiblement la même direction. Mais comme ces plis n'affectent pas les filons amphibolitiques discordants de Sermersût et Tôrnârssuk, les structures majeures ENE sont certainement prékétilidiennes. Sur la péninsule d'Ivigtut, ces structures ont été néanmoins reprises au cours de l'activité orogénique kétilidienne car les amphibolites discordantes y sont plissées et parfois granitisées.

Dans la partie septentrionale de Qôrnoq, les gneiss ont été également repris par les déformations kétilidiennes qui sont accompagnées, à Sánerut, par la mise en place de corps granitiques dans le coeur d'anticlinaux et parfois même de granites intrusifs.

\section{Résumé et conclusion}

Pendant l'activité orogénique kétilidienne, les roches sédimentaires et volcaniques du bassin d'Arsuk ont été déformées par trois phases importantes correspondant chronologiquement: (1) à des plis contemporains du développement de la schistosité; (2) à la déformation de ces structures par des plis accompagnés de clivage de crénulation; (3) à la déformation des structures précédentes.

La direction primitive des plis de la première phase oscille entre $\mathrm{N}-\mathrm{S}$ et NE-SW. Dans l'édifice volcanique, on observe de grands plis couchés vers l'Ouest. Dans les sédiments, les plis sont plus serrés et montrent la même tendance au déversement vers l'Ouest.

Lors des déformations suivantes, les horizons des schistes pyriteux et graphiteux ont donné naissance à des disharmonies importantes. La série kétilidienne a ainsi été divisé en deux complexes tectoniques qui se sont déformés de manière différente.

Les roches volcaniques ont été plissées en forme de grand synclinal de direction $\mathrm{NE}-\mathrm{SW}$ à $\mathrm{ENE}-\mathrm{WSW}$, légèrement déversé vers le Nord. Des zones de cisaillement, recoupant les couches, ont facilité les défor- 
mations. Dans les roches du groupe sédimentaire, on observe des plis de direction E-W à NW-SE, couchés vers le Nord et accompagnés de chevauchements plus ou moins importants. Les gros filons-couches intercalés dans les sédiments ont provoqué des disharmonies. Ils ont été divisés en écailles qui se sont également chevauchées.

La variation de la direction primitive des plis de la $2^{\mathrm{e}}$ phase $\left(045^{\circ}\right.$ à $080^{\circ}$ dans les roches volcaniques, $080^{\circ}$ à $110^{\circ}$ dans les sédiments de la côte orientale d'Arsuk $\varnothing, 145^{\circ}$ à $165^{\circ}$ dans les sédiments à Taylers Havn) est due à des disharmonies situées au niveau des horizons graphiteux et pyriteux.

On peut se demander si la $2^{\mathrm{e}}$ phase de plissement kétilidien n'est pas liée à la formation, dans le vieux socle d'une dépression à grand rayon de courbure de direction sensiblement ENE-WSW. Comme cette dépression s'accentuait, les séries kétilidiennes s'y écoulèrent en se plissant. Le déversement des plis et le sens des chevauchements les plus importants indiquent un mouvement d'écoulement de ces séries du Sud vers le Nord.

Le relèvement du socle dans la partie orientale de cette dépression (gneiss de la péninsule d'Ivigtut) donna naissance à la $3^{\mathrm{e}}$ phase de plissement. Des plis N-S ont perturbé les structures précédentes. Les déformations sont plus intenses dans les sédiments kétilidiens en bordure de la péninsule d'Ivigtut. Sur Arsuk $\emptyset$, les déformations consécutives à cette $3^{\mathrm{e}}$ phase ont eu pour effet d'accentuer la structure en baignoire de l'édifice volcanique (éventail des axes).

Les mouvements qui suivirent furent moins souples. Le relèvement du socle tout autour de la région d'Arsuk $\emptyset$ a donné naissance à la discordance structurale entre les quartzites de Taylers Havn et le socle. Dans la partie septentrionale d'Arsuk $\varnothing$, le redressement général des axes $\mathrm{N}-\mathrm{S}$ est sans doute lié à cette remontée du socle.

Les mouvements postérieurs furent de nature cassante. L'édifice kétilidien est alors resté solidaire du socle et a subi sensiblement les mêmes déformations que ce dernier. Cette phase de tectonique cassante, qui a débuté à la fin de l'activité orogénique, s'est poursuivie durant les périodes Gardar et post-Gardar.

Les datations absolues obtenues à partir de roches provenant des filons doléritiques discordants dans le vieux socle, métamorphisés à la fin du Protérozoīque inférieur, permettent de situer l'activité orogénique kétilidienne entre les valeurs extrêmes de $1910 \pm 45 \mathrm{~m}$. a. (mise en place des premiers filons basiques datés) et $1600 \mathrm{~m}$. a. correspondant au stade d'activité finale de la croûte du Groenland méridional (tableau 2). 


\section{Les déformations cassantes post-kétilidiennes}

\section{La période Gardar}

En même temps qu'il remontait dans les étages tectoniques supérieurs, l'édifice kétilidien était soumis à une érosion intense qui mis à jour ses parties les plus profondes et son soubassement prékétilidien. L'ensemble du bâti subit alors une extension qui s'est traduite par la formation d'un réseau complexe de joints.

Les produits de démantèlement constituèrent une nouvelle accumulation ouvrant le cycle des formations de Gardar, comprenant des roches supracrustales et abyssales, ces dernières caractérisant la province alcaline Gardar du Groenland méridional.

De cette accumulation de roches supracrustales, il ne subsiste que quelques reliques préservées dans des régions priviligiées comme le bassin faillé de Tunugdliarfik, situé à $130 \mathrm{~km}$ environ à l'ESE d'Arsuk $\varnothing$ et dans lequel se trouvent des produits détritiques d'origine continentale et des produits volcaniques constituant la Formation d'Eriksf jord (Poulsen, 1964, voir également Scharbert, 1963 et Wegmann, 1938).

Les failles majeures de direction WNW qui bordent ce bassin se poursuivent vraisemblablement sous l'inlandsis, jusque dans la région d'Ivigtut et limitent le "bloc faillé d'Ivigtut-Igaliko» (Berthelsen, 1962, planche 7). Ces failles ont une longue histoire et restèrent actives durant le Gardar (Henriksen, 1960).

En dehors de la région de Tunugdliarfik, les roches de Gardar sont représentées par des filons volcaniques et des roches ignées. Les recoupements entre ces différentes roches et les relations entre quelques-unes d'entre elles et les séries supracrustales de Tunugdliarfik ont permis d'établir une chronologie relative des principaux événements Gardar (Sørensen, 1966; Allaart, 1973).

Les datations absolues de roches ignées et filoniennes permettent de situer le début du magmatisme Gardar autour de $1275 \pm 25$ millions d' années (filon de lamprophyre dans la région d'Ivigtut; LARSEN, 1966) et la fin vers 1020 m. a. (intrusion d'Ilímaussaq; Moorbath et al., 1960; BridGWater, 1965). Les granites de Julianehåb datés par la méthode de $\mathrm{Rb} / \mathrm{Sr}$ (roche totale) indiquent un âge de $1776 \pm 37 \mathrm{~m}$.a. (van BreEMEN et al., 1974).

Sur Arsuk $\varnothing$, les roches Gardar ne sont représentées que par des filons volcaniques. L'étude des recoupements entre ces filons et les accidents tectoniques cassants a précisé la chronologie des déformations Gardar. Nous n'aborderons pas ici l'étude pétrographique des filons, celle-ci ayant été faite dans les travaux cités plus haut. La planche 14 montre la relation entre les filons et la tectonique cassante dans la région d'Arsuk $\varnothing$. 
Chronologie des déformations Gardar sur Arsuk $\varnothing$ Les chesauchements $N-S$ à $N N W$

Des mouvements de chevauchement se sont produits sur des plans de diaclases d'orientation $070^{\circ}$ à $120^{\circ}$, inclinés de $10^{\circ}$ à $35^{\circ}$ vers le Sud. Ils sont fréquents dans la partie occidentale d'Arsuk Ø. Les mouvements différentiels indiquent généralement un déplacement relatif du compartiment supérieur vers le Nord. Les stries de glissement ont une direction $150^{\circ}$ à $010^{\circ}$ (voir planche 18 ).

\section{Les diaclases $E N E$ à $E-W$ et les filons de lamprophyre}

Les filons de lamprophyre se sont mis en place dans des diaclases ouvertes appartenant à un système de direction $070^{\circ}$ à $090^{\circ}$, subverticales ou inclinées de $70^{\circ}$ à $80^{\circ}$ vers le Nord. L'épaisseur des filons varie entre $3 \mathrm{~cm}$ et $4 \mathrm{~m}$. Ils ont rarement été observés au-dessus d'une altitude de $400 \mathrm{~m}$. Certains filons, de 1 à $2 \mathrm{~m}$ d'épaisseur, ont pu être suivis sur une distance de $1,5 \mathrm{~km}$.

Ces lamprophyres recoupent le complexe alcalin d'Íka-Grønnedal (syénite à néphéline) au Nord d'Ivigtut (Emeleus, 1964). L'âge absolu d'un filon de lamprophyre de la région d'Ivigtut et appartenant au même essaim est de $1275 \pm 25$ m. a. (LArsen, 1966).

\section{Les filons à mégacristaux de feldspath et à xénolithes}

Des filons à mégacristaux de feldspath ("big feldspar dykes") et à xénolithes (p. 88), de direction $090^{\circ}$ à $100^{\circ}$, sont visibles sur l'îlot Mitdlûvfik, à la pointe Nord-Ouest d'Arsuk $\varnothing$ et au Sud de Blålershavn. Ces filons sont recoupés sur Arsuk $\varnothing$ par les filons $\mathrm{BD}_{1}$ et $\mathrm{BD}_{2}$; leur recoupement avec les lamprophyres n'est pas visible. Une étude très détaillée des mégacristaux de plagioclase se trouvant dans les intrusions précambriennes du Groenland méridional a été faite par Bridgwater \& HARRY, 1968.

Première génération de filons de diabase à olivine $B D_{0}$

Un filon de diabase à olivine $\left(\mathrm{BD}_{0}\right)$, de direction $100^{\circ}$, se trouve dans la région Sud-Est d'Arsuk $\varnothing$. Il est recoupé et déplacé par un décrochement $\mathrm{N}-\mathrm{S}$. Aucun recoupement entre ce filon et les filons à xénolithes n'est visible sur Arsuk $\varnothing$.

\section{La deuxième génération de diabase à olivine $\left(B D_{1}\right), E N E$}

Un gros filon de diabase à olivine, $\mathrm{BD}_{1}$, d'une centaine de mètres d'épaisseur, incliné de $60^{\circ}$ à $70^{\circ}$ vers le Sud, est visible sur la côte Nord d'Arsuk Ø. Il se poursuit sur l'îlot Mitdlûvfik où il recoupe les filons à xénolithes et les lamprophyres. On le retrouve sur la péninsule d'Ivigtut, 
au Nord de Webers Havn, où il recoupe un filon $\mathrm{BD}_{0}$. Il semble appartenir à la même génération que le gros filon $\mathrm{BD}_{2}$ appelé "the Master Dyke " qui traverse l'intrusion alcaline de Grønnedal (Emeleus, 1964, fig. 16).

\section{Les décrochements $N-S \grave{a} N N E$}

Des mouvements de décrochement N-S à NNE sont particulièrement marqués dans le secteur de Taylers Havn. Sur Arsuk $\emptyset$, les décrochements du lac $630 \mathrm{~m}$ appartiennent sans doute au même système. On observe également un rejeu de la zone de cisaillement d'Igánaq-lac $130 \mathrm{~m}$, au Sud-Est d'Arsuk $\varnothing$ (fig. 45).

Les mouvements différentiels indiquent généralement un déplacement vers le Sud du compartiment oriental. Des décrochements de ce type recoupent nos filons $\mathrm{BD}_{1}$ sur la péninsule d'Ivigtut.

Les diaclases $N E$ et la troisième génération de flons de diabase à olivine $\mathrm{BD}_{2}$

Les filons de la troisième génération de diabase à olivine, $\mathrm{BD}_{2}$, se sont mis en place dans des diaclases NE subverticales, réparties assez régulièrement sur Arsuk $\varnothing$.

\section{Les filons de trachyte ENE}

Les filons de trachyte de direction ENE sont sensiblement parallèles aux lamprophyres. Ils recoupent le décrochement $\mathrm{N}-\mathrm{S}$ du lac $130 \mathrm{~m}$, au Sud-Ouest d'Arsuk $\varnothing$. Sur la péninsule d'Ivigtut, ils recoupent Jes $\mathrm{BD}_{2}$.

\section{Les décrochements et failles $E N E$ à $E-W$}

Des failles ENE, subverticales, se sont produites après la mise en place des $\mathrm{BD}_{2}$, comme on peut l'observer sur la côte Nord d'Arsuk $\varnothing$. A ces failles succèdent des décrochements de même direction. Les mouvements différentiels indiquent généralement un déplacement du compartiment Nord vers l'Ouest. Lors de ces mouvements, les anciens plans de discontinuité (failles, diaclases, zones de discontinuité, cisaillements) ont été souvent réutilisés. Des décrochements appartenant au même système se retrouvent à Taylers Havn.

\section{Les mylonites $E-W$ à ENE}

Les peines tardives d'albite et de carbonate. Sur les côtes Nord et Est d'Arsuk Ø, à Mitdlûvfik et à Taylers Havn, les roches sédimentaires et volcaniques, ainsi que les filons de lamprophyre et de trachyte, sont recoupés par des zones mylonitiques imprégnées de carbonate, de teinte brun roux. L'albite y est généralement associée. Ce minéral a parfois cristallisé dans des fissures ouvertes ou des géodes. Dans certains cas, 


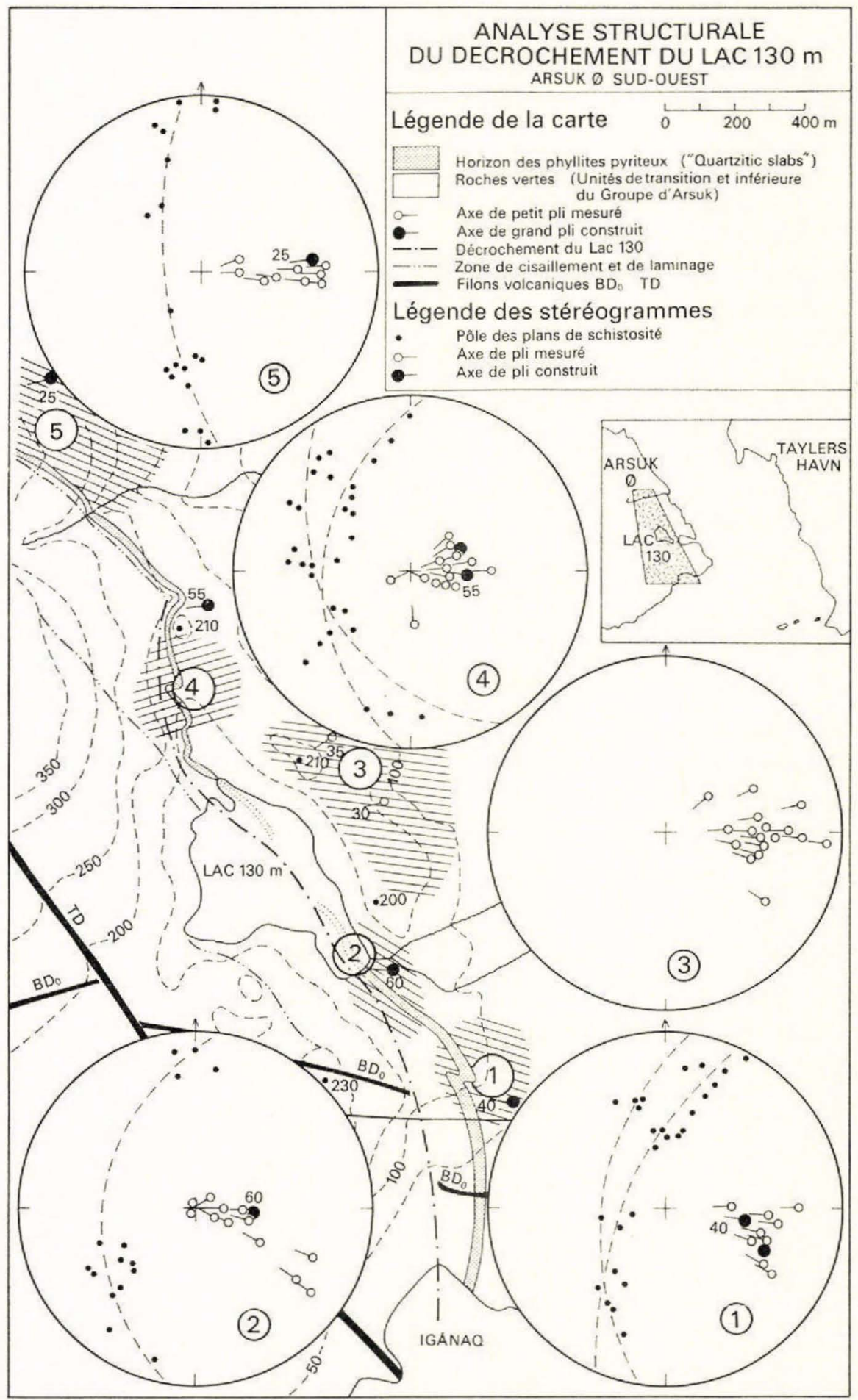

Fig. 45. Torsion des axes de plis kétilidiens par le décrochement du lac 130 (plis "verticalisés »). 
les roches traversées par ces zones semblent avoir été assimilées par les produits carbonatés et albitiques qui les remplacent.

L'examen microscopique montre que les roches carbonatisées sont recoupées par un réseau de fissures très serrées, ramifiées et remplies d'albite, de carbonate, de chlorite et de minerai de fer (goethite, lépidocrocite, pyrite; déterminations faites par Berrangé, GGU). Dans certains phyllites du versant Nord d'Arsuk $\varnothing$, on reconnaît en outre de la biotite brune presque opaque, parfois de l'aégyrine (détermination aux rayons $\mathrm{X}$ ) ou encore de la riebeckite. Les remplissages leucocrates sont souvent visibles à l'oeil nu. Dans les quartzites de Taylers Havn, des fissures de ce type sont remplies d'albite et de microcline. Ces minéraux peuvent s'infiltrer dans toutes les zones de discontinuité des roches et les imprégner.

Ces phénomènes sont sans doute liés à la mise en place du granite alcalin d'Ivigtut et à la minéralisation accompagnant la cristallisation de la cryolite (BERTHELSEN, 1962). Les émanations ont suivi de préférence les anciennes zones de discontinuité dans lesquelles les minéralisations métalliques ne sont pas rares, notamment dans le secteur de Taylers Havn (chalcopyrite, azurite, galène).

Les résultats obtenus par les datations absolues de pegmatites du granite d'Ivigtut varient entre $1160 \pm 40$ et $1230 \pm 40 \mathrm{~m}$. a. (BRIDGWATER, 1965).

\section{Les déformations post-Gardar}

Les diaclases $N N W$ et les filons de diabase à olivine TD

Des diaclases subverticales, de direction $145^{\circ}$ à $155^{\circ}$, sont bien marquées dans la région occidentale d'Arsuk Ø. Elles appartiennent au même système que les diaclases ouvertes dans lesquelles se sont mis en place les filons de diabase à olivine, appelés aussi "trap diabase 》(TD). Ces filons font partie d'un essaim localisé le long de la côte Ouest du Groenland méridional (Muller, 1962) et qui est constitué par cinq générations de filons sensiblement du même type pétrographique, dont trois sont représentés sur Arsuk $\varnothing$ (fig. 46). Ces filons ne sont pas toujours verticaux. Ils s'inclinent parfois légèrement vers l'Est ou vers l'Ouest. Certains d'entre eux ont été suivis sur plus de $100 \mathrm{~km}$. Leur mise en place est peut-être liée à une flexure continentale.

L'âge absolu d'un filon de lamprophyre, contemporain de la mise en place des TD à Frederikshåb, (Walton, 1966) est de 164 m. d'années, c'est-à-dire mésozoïque (LARSEN, 1966).

\section{Les mouvements de réajustement}

Les filons de diabase à olivine TD ne sont recoupés par aucun accident cassant important. Sur la côte Sud-Est d'Arsuk $\varnothing$ cependant, le filon 


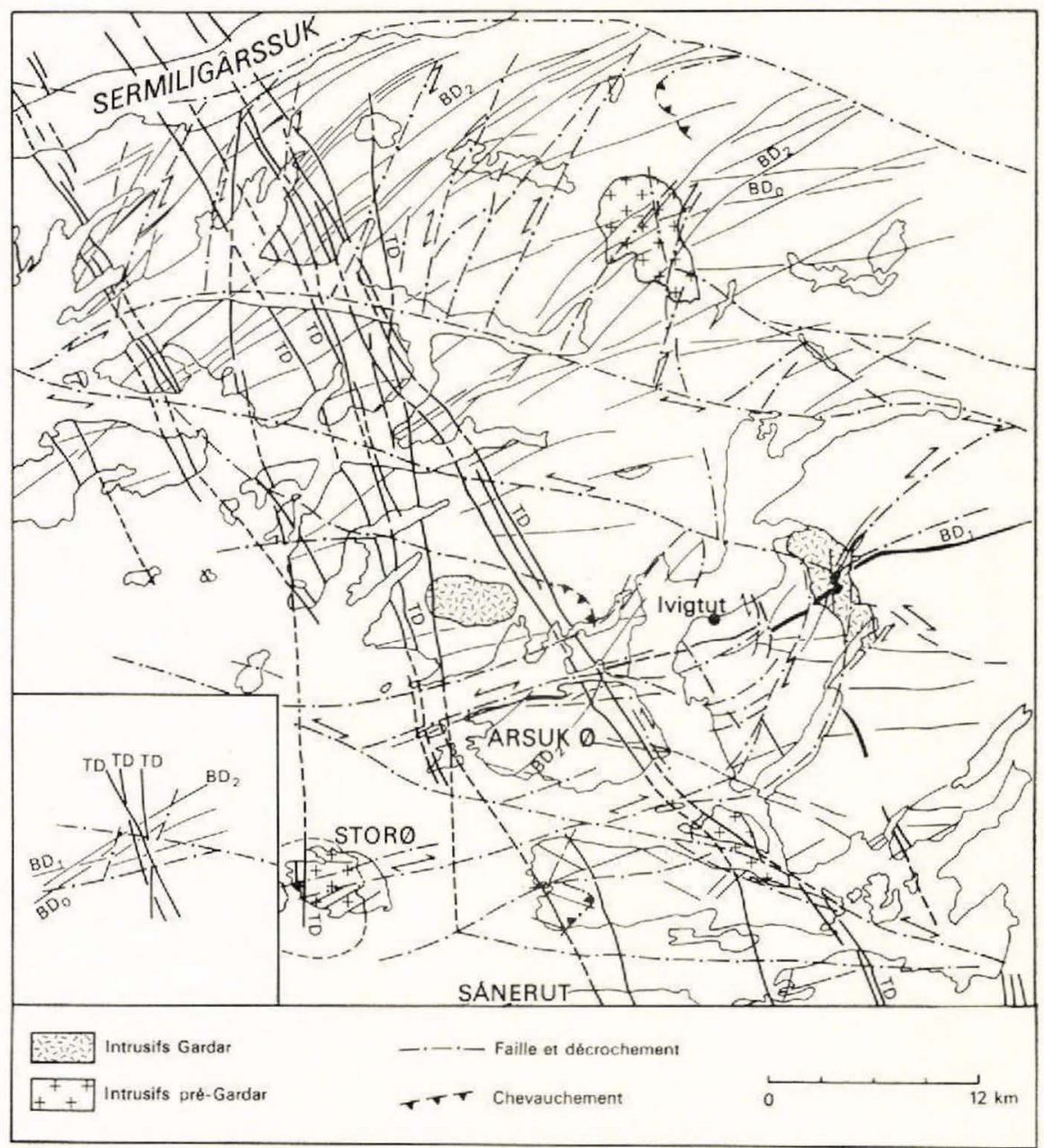

Fig. 46. Fracturation tardikétilidienne et Gardar dans la région d'Ivigtut. Volcanisme filonien Gardar (diabase à olivine appartenant à 3 générations au moins) et postGardar (diabase à olivine appartenant à 4 générations $\mathrm{TD}_{00}, \mathrm{TD}_{0}, \mathrm{TD}_{1}, \mathrm{TD}_{2}$ ).

$\mathrm{TD}_{2}$ oriental est affecté par de légers mouvements de réajustement mis en évidence par des diaclases striées subhorizontales sur lesquelles les stries, de direction $030^{\circ}$, indiquent un déplacement vers le NNE du compartiment supérieur.

\section{Conclusion}

Les filons volcaniques se sont mis en place dans les diaclases ouvertes appartenant à plusieurs systèmes représentés sur tout le territoire étudié et au-delà. 
Les décrochements et failles $\mathrm{E}-\mathrm{W}$ sont très importants. Ils ont rejoué à plusieurs reprises. Les observations faites dans les régions avoisinantes montrent que certains existaient déjà dans le vieux socle avant la mise en place des filons doléritiques actuellement métamorphisés en amphibolite (HenRIKSEN, 1960). Ils divisent le Groenland méridional en gigantesques amygdales en formes de "poissons", elles-mêmes divisées en amygdales plus petites. La région d'Arsuk $\varnothing$ correspond à l'une d'entre elles. Le déplacement des amygdales les unes par rapport aux autres a provoqué des déformations secondaires à l'intérieur de chacune d'elles. Ainsi la formation des accidents N-S est probablement la conséquence de mouvements différentiels qui se sont produits suivant les grandes zones de discontinuité $\mathrm{E}-\mathrm{W}$. 


\section{LE BASSIN VOLCANO-SEDIMENTAIRE D'ARSUK ET SON EVOLUTION METAMORPHIQUE}

\section{Dépôt des séries volcano-sédimentaires}

Les formations sédimentaires et volcaniques de la région d'Arsuk $\varnothing$ ont une épaisseur voisine de $5500 \mathrm{~m}$. Le groupe sédimentaire, dont la base est recoupée par une discordance tectonique, débute, avec la Formation de Taylers Havn, par le dépôt de grès arkosiques devenant de plus en plus purs vers le haut de la série. Il est possible que des sédiments semi-pélitiques carbonatés aient précédé le dépôt des grès (horizon d' Evqitsut). Au-dessus des grès vient une série grésocarbonatée alternant avec des sédiments grésoargileux. Ces derniers deviennent de plus en plus argileux vers le haut et renferment quelques niveaux franchement carbonatés. La formation se termine par des sédiments argilographiteux riches en pyrite avec quelques minces intercalations de grès arkoses et roches carbonatées.

Avec la Formation d'Inugsugtût, les roches deviennent à nouveau plus gréseuses. Les grès alternant assez régulièrement avec des sédiments argileux, parfois pyriteux. Les structures de glissements sous-marins indiquent une certaine instabilité dans le bassin.

Cette alternance de grès et d'argile se poursuit dans la Formation d'Isua, mais les niveaux argileux deviennent plus épais et plus fréquents. Le groupe sédimentaire se termine enfin par des sédiments argileux très pyriteux renfermant des lits de cherts lenticulaires.

Avec le groupe volcanique, la nature du remplissage du bassin d'Arsuk se modifie complètement. Emission de lave subaquatique, donnant naissance à des entassements considérables de pillow-lavas, alternant avec des laves massives basiques qui ne présentent aucune structure de refroidissement rapide. La mise en place des laves massives est discutable: l'hypothèse d'émissions subaériennes alternant avec des laves sous-marines impliquerait une faible hauteur d'eau dans le bassin mais par contre une forte subsidence. Dans une autre hypothèse les laves massives pourraient correspondre à des coulées sous-marines protégées du refroidissement par une couverture de pillows.

Des brèches volcaniques, des lapillis, des tufs et des cendres accompagnent les émissions de lave. On note également des brèches volcaniques 
remaniées. Des ultrabasites s'intercalent entre les coulées volcaniques. $\mathrm{Au}$ début du volcanisme, on observe une récurrence de sédiments argilopyriteux avec lits de cherts lenticulaires.

Lors du volcanisme, les roches du groupe sédimentaire ont été intrudées par des filons-couches parfois très épais. Certains semblent se poursuivre sur de grandes distances en restant situés approximativement au même niveau lithostratigraphique. L'un d'eux forme la limite entre les Formations d'Inugsugtût et d'Isua.

Des petits filons basiques discordants se sont mis en place à la fin de l'épisode volcanique. Il ne subsiste dans le bassin d'Arsuk aucune relique de roche ayant pu surmonter le groupe volcanique.

Cette succession de roches sédimentaires et volcaniques caractérise le bassin kétilidien d'Arsuk. Lors de l'activité orogénique kétilidienne, ces roches ont subi des transformations marquées par la cristallisation de minéraux. Certains d'entre eux permettent d'établir une chronologie entre les déformations et les métamorphismes. L'âge des néoformations a été défini par rapport aux différentes surfaces $S$ reconnues dans les roches.

Les cristallisations

Les micas blancs et les chlorites

Dans les roches sédimentaires, les muscovites se disposent suivant la schistosité $S_{1}$. Elles ont subi ensuite une réorientation parallèlement au clivage $S_{2}$ et même au clivage $S_{3}$ qui accompagne parfois les plis de la $3^{\mathrm{e}}$ phase. Il en est de même pour les chlorites. Les micas blanes provenant de la séricitisation de matériel feldspathique très cataclasé s'orientent aussi suivant ces directions.

Les chlorites sont présentes dans la plupart des roches volcaniques. Elles proviennent de la transformation des amphiboles dans les roches affectées par la schistosité. Elles se disposent parallèlement aux plans schisteux et enserrent des chapelets de leucoxène. On la trouve aussi en remplissage des fentes pennées qui se forment parfois dans la croûte des pillows déformés par la schistosité (p. 62).

\section{Les feldspaths}

Dans les quartzites de la Formation de Taylers Havn, on observe une cristallisation de feldspath potassique qui se dispose parallèlement aux plans de partage de la roche (clivage $S_{2}$ ou/et schistosité $S_{1}$, planche $1 \mathrm{c}$, p. 23). Dans les phyllites, les feldspaths potassiques qui s'allongent entre les feuillets micacés (fig. 47 et p. 31) sont déformés par la $3^{\mathrm{e}}$ phase de plissement.

Dans les schistes granuleux feldspathiques, la cristallisation des andésines contenant des inclusions plissées est postérieure à la schistosité 
$S_{1}$ ou au clivage $S_{2}$ (fig. 11 et p. 35). Ces minéraux sont déformés par la $3^{\mathrm{e}}$ phase de plissement.

Dans les roches volcaniques déformées par la schistosité, les plagioclases calciques primaires (andésine) sont réduits en grains très saussuritisés avec néoformation d'oligoclase ou d'albite (p. 57). Dans les fentes pennées citées plus haut, il y a aussi de l'albite.

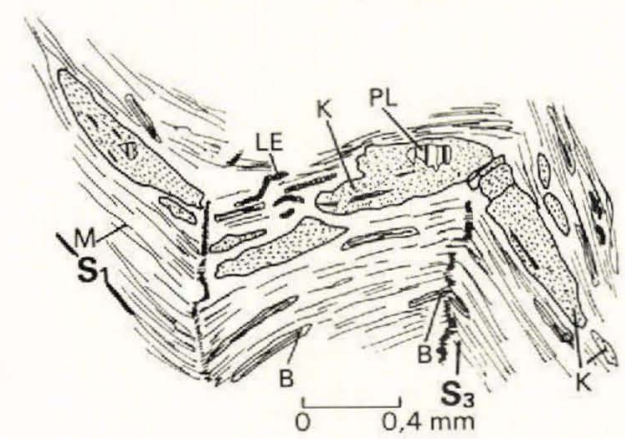

Fig. 47. Micrographie d'un phyllite quartzofeldspathique de l'horizon des quartzites dolomitiques à Taylers Havn. Les feldspaths potassiques (k) allongés parallèlement à la schistosité $S_{1}$ sont cassés dans des charnières de plis de la $3^{\mathrm{e}}$ phase accompagnés d'un clivage $S_{3}$ assez peu marqué. M: muscovite. LE: leucoxène. B: biotite. PL: plagioclase. (GGU 56576).

\section{Les amphiboles}

Dans les calcaires dolomitiques de Taylers Havn, les gerbes de trémolite se développent de préférence dans les plans de schistosité mais elles les recoupent parfois perpendiculairement (p. 28). Les lits amphiboliques (trémolite) ont été boudinés lors de la 3 e phase de plissement (p. 110). En bordure des boudins, les trémolites sont biotitisées.

Dans les roches volcaniques non déformées à grain moyen ou grossier, on observe la transformation de l'hornblende brune (primaire?) en actinote. Ces deux amphiboles sont chloritisées. Dans les roches déformées par la schistosité, l'amphibole brune, quand elle est encore présente, est très cataclasée. Les actinotes sont "déchirées" et leurs fragments ont tendance à s'orienter parallèlement à la schistosité. Par endroit, on observe une recristallisation d'actinote fibreuse parallèle à la schistosité (p. 76).

\section{Les épidotes}

Les épidotes sont présentes dans presque toutes les roches sédimentaires et volcaniques. On distingue des pistacites, des clinozoïsites et des orthites. La zoisite est rare. Le zonage pistacite-clinozoïsite est fréquent. Les épidotes appartiennent à plusieurs générations. Il n'a pas été possible 
de replacer chacune d'elles dans le cadre structural. L'orthite est fréquemment cassée et ressoudée par une pistacite ou une clinozoïsite.

Dans les gneiss de la côte Nord d'Arsuk Ø, la formation de la pistacite est postérieure à celle de la biotite poecilitique (p. 83). Dans les phyllites et les schistes de Taylers Havn, les épidotes ont été cassées lors du plissement $\mathrm{N}-\mathrm{S}$ de la $3^{\mathrm{e}}$ phase. Toutefois, certaines d'entre elles, qui recoupent des petits plis N-S, sont postérieures à cette déformation (p. 31).

Dans les pillows, la cristallisation des épidotes sphérolitiques est postérieure au premier stade de transformation de la croûte (p. 59). Ces épidotes sont cataclasées dans les roches déformées par la schistosité (p. 62). D'autres épidotes sont plus tardives car elles ont cristallisé dans les fentes pennées.

\section{Les biotites}

Dans les roches sédimentaires, la biotite est répartie très irrégulièrement. Dans les roches volcaniques, elle est rare ou absente. Son pléochroïsme varie suivant la nature des roches dans lesquelles elle se trouve. Elle est verte dans les schistes granuleux et certains phyllites $(\alpha=$ jaune clair; $\gamma=$ vert olive). Dans les calcaires et quartzites dolomitiques, elle est peu colorée $(\alpha=$ incolore; $\gamma=$ brun-jaune clair). Dans les schistes sombres pyriteux et graphiteux, elle est brune $(\alpha=$ brun clair; $\gamma=$ brun roux).

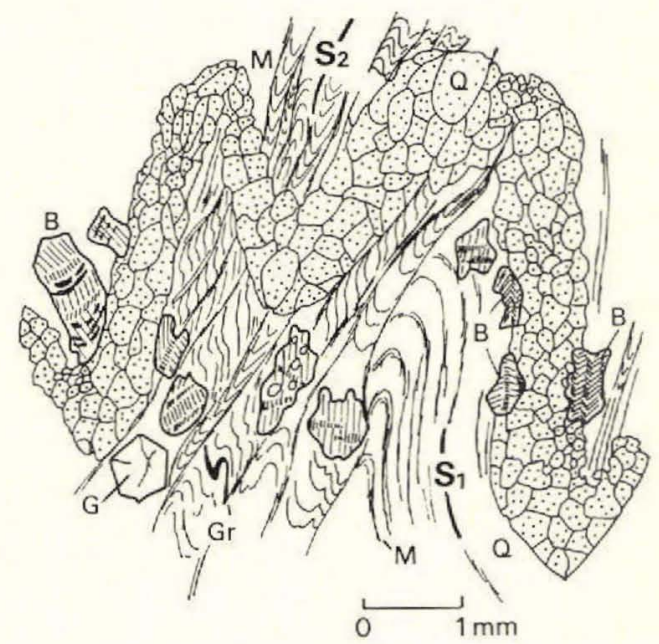

Fig. 48. Micrographie d'un schiste sombre à grenat et biotite de la Formation de Taylers Havn. Inugsuk. La schistosité $S_{1}$ ainsi qu'une veinule de quartz (Q) sont déformées par des plis microscopiques accompagnés de clivage $S_{2}$. La biotite poecilitique (B), postérieure au clivage, renferme des inclusions de graphite (Gr) disposées parallèlement à l'ancienne schistosité. La biotite et le grenat $(G)$ ont été déformés ensuite lors d'un rejeu des plans de clivage. (GGU 54882). 


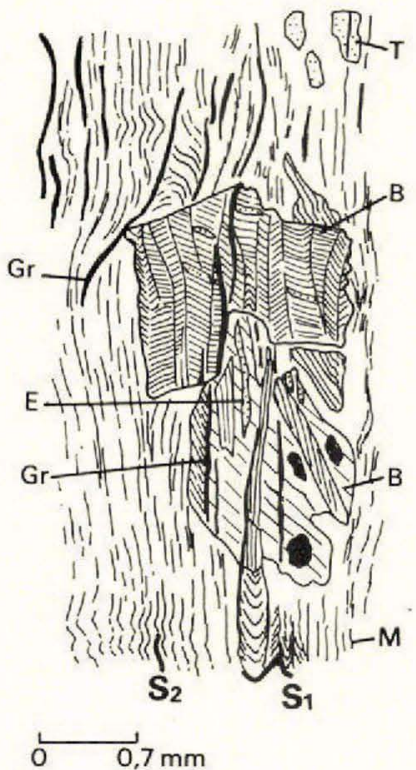

Fig. 49. Micrographie d'un schiste sombre à biotite brune. Inugsuk. Développement de biotite brune (B), poecilitique, cicatrisant les plans de schistosité $S_{1}$ et de clivage $S_{2}$ soulignés par la muscovite (M). Cette biotite, qui renferme des inclusions de graphite $(\mathrm{Gr})$, a été déformée lors de la $3^{\mathrm{e}}$ phase, par un rejeu des plans de clivage. L'épidote (E) est en intercroissance avec la biotite. La tourmaline (T) a aussi des inclusions opaques.

On distingue deux types structuraux :

Les petites biotites, de quelques dizaines de millimètres de diamètre, se disposent comme les muscovites parallèlement à la schistosité $S_{1}$. Ces biotites sont syncinématiques par rapport à la première phase de déformation.

Les grandes biotites, de 1 à $3 \mathrm{~mm}$ de diamètre, ont cristallisé dans les plans de clivage $S_{2}$ et les cicatrisent. Ces biotites sont postcinématiques par rapport à la $2^{\mathrm{e}}$ phase (p. 37).

Le graphite, disposé dans les anciens plans de schistosité $S_{1}$ ou de clivage $S_{2}$, se retrouve sous forme d'inclusions dans ces grandes biotites (fig. 48 et 49). Ces biotites ont été ensuite déformées lors de la 3 e phase. A Taylers Havn, les grandes biotites sont plissées ou granulées dans les charnières de plis N-S alors que dans les flancs de ces plis, elles sont cisaillées par rejeu des plans de clivage. Dans les gneiss de la côte Nord d'Arsuk $\varnothing$, de larges biotites brunes se sont développées poecilitiquement dans une matrice très cataclasée (fig. 28 et p. 83). Si ces biotites appartiennent, à la même génération que celles de Taylers Havn, ce qui est probable, la cataclase principale de ces gneiss serait antérieure au plissement de la $3^{\mathrm{e}}$ phase. 
Dans les roches volcaniques, la biotite est rare. Dans les filonscouches, elle est postérieure à la cataclase principale de la roche (p. 76). On la trouve disséminée en minuscules cristaux dans quelques roches gabbro-dioritiques.

\section{Les grenats}

Les grenats, qui se trouvent dans plusieurs horizons sédimentaires, peuvent être divisés en deux groupes:

Les petits grenats, idiomorphes, de quelques centièmes de millimètres de diamètre, sont disposés dans des lits qui semblent correspondre à la stratification. Au microscope, ils sont incolores.

Les grenats poecilitiques, de 0,5 à $1 \mathrm{~cm}$ de diamètre, recoupent la schistosité et le clivage. Au microscope, ils sont incolores; à l'oeil nu, ils ont une teinte rose ou rougeâtre.

Ces deux groupes peuvent appartenir à la même génération. A Taylers Havn, les grenats poecilitiques se sont développés en mimant les structures telles que la schistosité $S_{1}$ et le clivage $S_{2}$ (fig. 50). Ils semblent également
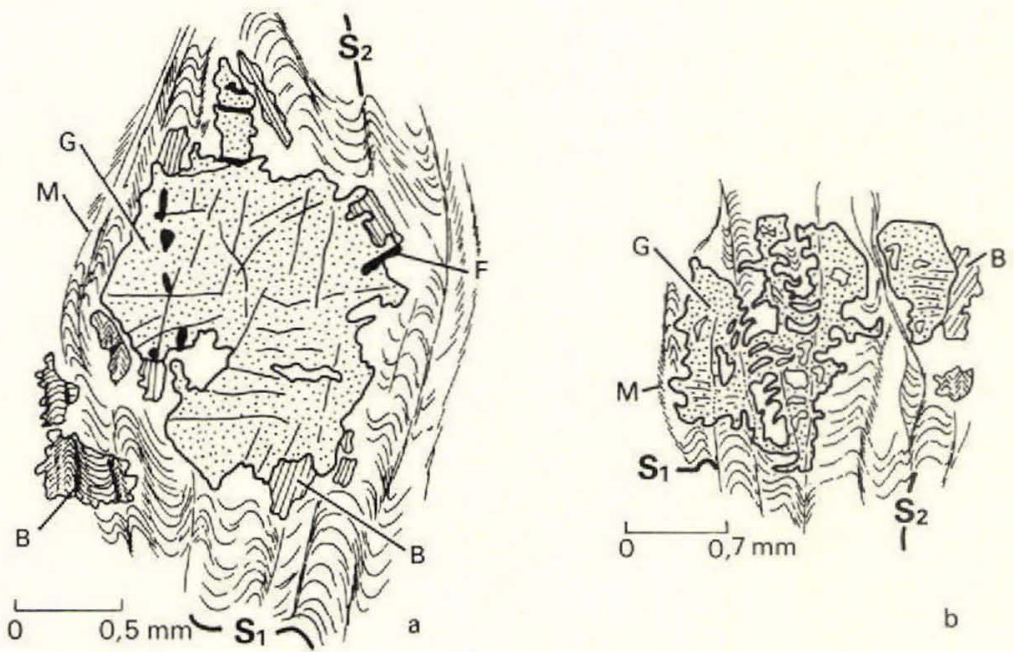

Fig. 50. a. Micrographie d'un schiste grenatifère déformé. Inugsuk. Texture oeillée du schiste. Le grenat (G) est postérieur à $S_{1}$ et $S_{2}$. (GGU 56579). $b$. Micrographie du même schiste. Développement poecilitique de grenat mimant les anciennes structures $S_{1}$ et $S_{2}$. M: Muscovite. B: biotite. F: minerai.

postérieurs à la formation des grandes biotites (planche $3 \mathrm{~b}$ ). Ils ont été déformés lors du plissement de la $3^{\mathrm{e}}$ phase. Dans les flanes des plis, ils ont parfois roulé en donnant une texture oeillée aux schistes qui les contiennent (ZwART, 1960). 
Metamorphisme associé à la $1^{\text {re }}$ phase de déformation

Les associations minérales rencontrées dans les sédiments montrent que le métamorphisme associé à la 1re phase de plissement (synchrone de la schistosité) correspondait au faciès des schistes verts, subfaciès de la biotite-chlorite de TURNER \& Verhoogen (1951).

Dans les roches volcaniques, basiques et ultrabasiques, on note déjà une instabilité des hornblendes brunes, des plagioclases calciques et de l'olivine avant l'apparition de la schistosité. Cette instabilité s'est traduite par la néoformation d'actinote, d'épidote, d'albite oligoclase, antigorite, talc et carbonate. L'apparition de la schistosité est marquée par une néoformation de chlorite aux dépends des amphiboles, une leucoxénisation de l'ilménite et la formation d'albite renfermant parfois des inclusions d'actinote.

\section{Les conditions métamorphiques entre la $1^{\text {re }}$ et la $3^{\text {e }}$ phase}

Les néoformations les plus apparentes qui se sont produites entre la 1 re et la $3^{\mathrm{e}}$ phase de plissement n'ont été observées qu'à proximité du socle, c'est-à-dire à Taylers Havn, sur la côte Nord d'Arsuk $\varnothing$ ainsi que sur les îlots Sud-Est. On distingue:

des plagioclases calciques (andésine) contenant des reliques de plis, des biotites brunes en larges cristaux, postérieures à la $2^{\mathrm{e}}$ phase, des grenats rosés en larges cristaux, postérieurs à la $2^{\mathrm{e}}$ phase et peutêtre aux biotites, des trémolites incolores, postérieures à la $1^{\text {re }}$ phase, car certaines d'entre elles se disposent en travers de la schistosité des calcaires dolomitiques, des épidotes qui sont postérieures à la biotite.

La présence de ces minéraux, et plus particulièrement de l'andésine, indique une augmentation du métamorphisme (faciès de l'amphibole) entre la 1 re et la $3^{\mathrm{e}}$ phase de plissement. Cette augmentation de métamorphisme est localisée à la bordure du socle. Les seules néoformations observées dans l'édifice volcanique après la formation de la schistosité sont de minuscules et rares biotites ainsi que des fibres très ténues d'actinote. Ce métamorphisme peut correspondre à celui qui a affecté le socle après la mise en place des filons doléritiques AD. Nous rappelons ici que ces filons basiques, discordants dans le socle, ont été métamorphisés dans le faciès de l'amphibole sur la péninsule au Nord d'Arsuk $\varnothing$ (p. 89). Les recristallisations observées dans les roches kétilidiennes en bordure du socle seraient donc une conséquence du métamorphisme régional qui a affecté le socle entre la $1^{\text {re }}$ et la $3^{\mathbf{e}}$ phase de déformation kétilidienne. 
Le plissement de la $3^{e}$ phase

Lors de ce plissement, les minéraux précédemment décrits ont été déformés. Ils n'ont pas subi de transformation. On observe une réorientation mécanique de la muscovite dont les feuillets se disposent parallèlement aux plans de clivage qui accompagnent parfois les plis $\mathrm{N}-\mathrm{S}$ de la $3^{\mathrm{e}}$ phase. Les granules de leucoxène présentent aussi la même disposition.

\section{Recristallisations postérieures à la 3 e phase}

Dans les phyllites et les schistes de Taylers Havn, on assiste à une véritable remobilisation siliceuse après le plissement de la $3^{\mathrm{e}}$ phase. Le quartz s'infiltre dans toutes les zones de discontinuité des roches telles que les anciens plans $S_{1}$ ou $S_{2}$ et même $S_{3}$ (p. 31). L'extinction roulante des quartz montre que ce minéral a subi ensuite une nouvelle cataclase probablement liée à une phase de tectonique cassante tardikétilidienne ou Gardar. La chloritisation des grenats et parfois celle des biotites est postérieure à la $3^{\mathrm{e}}$ phase (p. 38).

Ces cristallisations tardives marquent la dernière étape des transformations intimes des roches kétilidiennes. Désormais, la cristallisation des minéraux sera limitée à quelques zones de broyage appartenant à la tectonique cassante. Les minéralisations (carbonate, albite, pyroxène et amphibole sodique ainsi que les minéralisations métalliques) associées à la mise en place de divers massifs plutoniques intrusifs ne s'écarteront plus guère de ces zones de discontinuité qui représentent les nouvelles voies de migration des éléments dans l'édifice kétilidien devenu rigide. 


\section{CORRELATIONS ENTRE}

\section{LES ROCHES KETILIDIENNES D'ARSUK $\varnothing$ ET DE GRÆNSELAND-MIDTERNÆS}

Dans la région de Grænseland-Midternæs, au Nord d'Arsuk Bræ, on retrouve des roches supracrustales qui se poursuivent d'ailleurs au Sud d'Arsuk Bræ presque sans interruption jusqu'à Sánerut (fig. 2).

On distingue à la base un Groupe de Vallen, sédimentaire, surmonté d'un Groupe de Sortis, volcanique à Midternæs et volcano-sédimentaire à Grænseland. Le Groupe de Qipisarqo, qui surmonte l'ensemble, n'apparaît qu'au Sud d'Arsuk Bræ. Il est formé de roches sédimentaires, dont un conglomérat très épais à la base, et de roches volcaniques (Higgins \& Bondesen, 1966).

Dans les régions de Grænseland-Midternæs et d'Arsuk $\varnothing$, on observe une succession de roches sédimentaires et volcaniques. Des corrélations faites entre ces deux régions éloignées seulement de 30 à $40 \mathrm{~km}$ mettent en évidence des différences notables de faciès dans les roches sédimentaires et des biseaux stratigraphiques importants, notamment dans les roches volcaniques.

Les documents mis aimablement à notre disposition par BoNDESEN (1970), qui a étudié la région de Grænseland, nous permettent de tenter les rapprochements mentionnés sur la fig. 51 . 

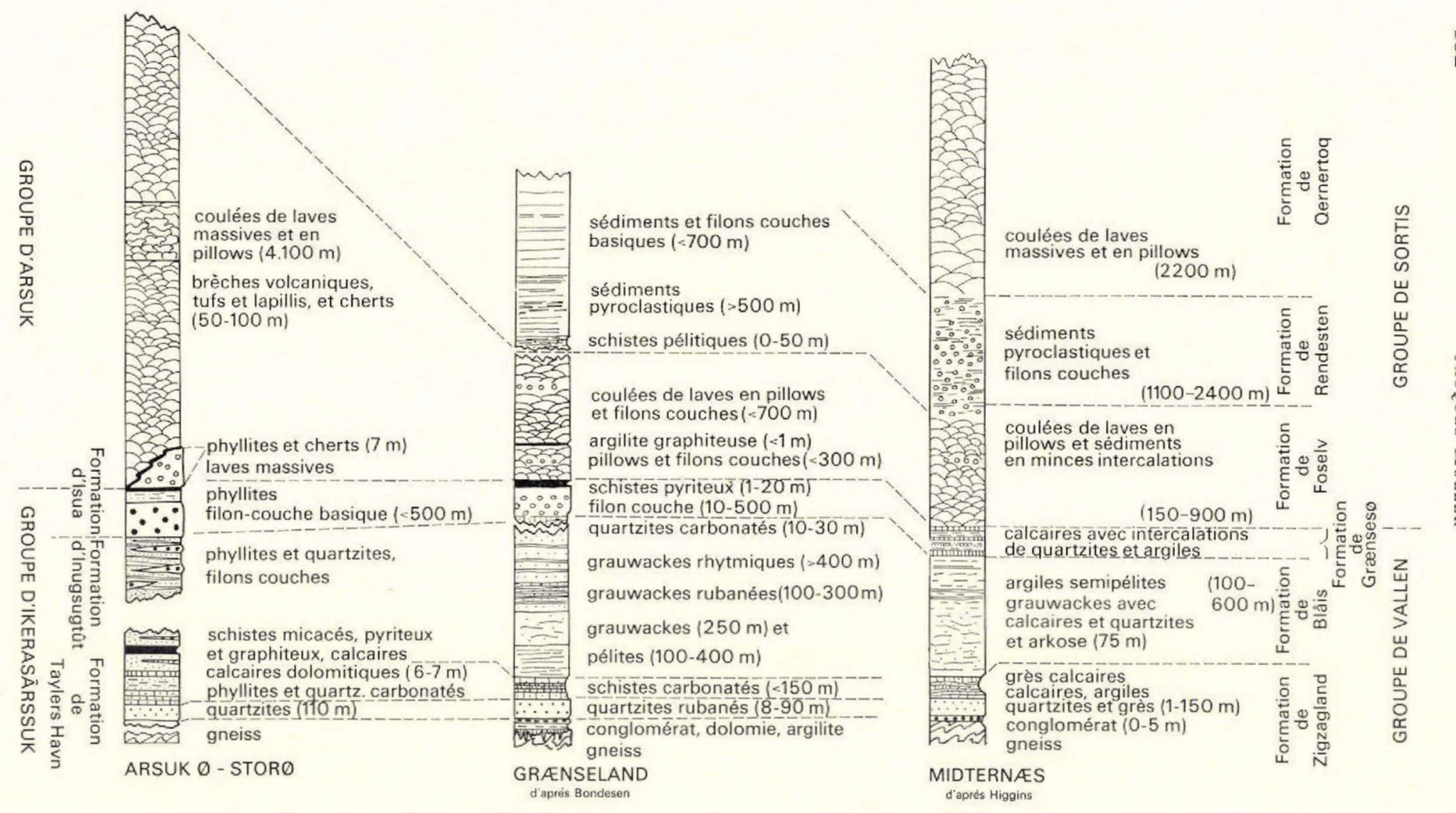

Fig. 51. Corrélations lithostratigraphiques proposées entre les régions d'Arsuk et Grænseland-Midternæs. 


\section{REMERCIEMENTS}

J'exprime ici ma profonde gratitude à mon maître le Professeur C. E. Wegmann, qui m'a appris mon métier et m'a guidé dans mes premiers pas sur cette terre groenlandaise qu'il connaissait bien.

Je remercie le Directeur du Service Géologique du Groenland, K. Ellitsgaand-Rasmussen, pour tous les moyens qu'il mit à ma disposition, tant au cours des expéditions au Groenland qu'au laboratoire à Copenhague et pour sa permission de publier ce travail.

La base topographique des cartes a été fournie par l'Institut Géodésique du Danemark (numéro de permission: A. 649/72).

Le Professeur A. Berthelsen, de l'Institut for Almen Geologi à Copenhague mit tout en oeuvre pour la réussite des deux expéditions en 1957 et 1958 dont je garde un souvenir inoubliable. Il suivit ensuite de près mes travaux et m'apporta sans cesse de nouvelles informations sur l'évolution des idées relatives à la géologie du Groenland méridional.

Le Professeur M. BurrI, du Laboratoire de Géologie de l'Université de Lausanne, a débattu avec moi les questions délicates que je me posais au fur et à mesure de la rédaction finale pendant l'hiver 1965-1966.

Le Professeur M. Vuagnat, du Laboratoire de Minéralogie de l'Université de Genève, m'a aidé à réaliser une étude détaillée des formations volcaniques d'Arsuk $\varnothing$.

Je dois au Professeur J. P. Schaer, Directeur de l'Institut de Géologie de l'Université de Neuchâtel, d'avoir pu achever ce travail. Il assuma la tâche de reprendre la direction de cette thèse après le départ du Professeur C. E. Wegmann.

Mes remerciements vont également à mes amis J. H. AllaArt, S. N. Ayrton, E. Bondesen, N. Henriksen, S. B. Jensen, F. Persoz, T. G. R. Pulvertaft et M. Weidmann pour les nombreuses discussions scientifiques et amicales que j'ai eues avec eux pendant les expéditions au Groenland.

Je n'oublie pas la collaboration de mon ami Claude Froidevaux avec lequel j'ai effectué les levés géologiques de la région d'Arsuk $\varnothing$ et de Storø en 1957 et 1958.

Par ses nombreux conseils et son étroite collaboration, ma femme assura la présentation de cet ouvrage. Je lui exprime ici toute ma reconnaissance. 


\section{LISTE BIBLIOGRAPHIQUE}

AllaA rT, J. H. 1973: Geological map of Greenland 1:100 000 Julianehåb 60 V.2 Nord. Descriptive text. Grønlands Geologiske Undersøgelse. (also Meddr Gronland $\left.192^{4}\right), 41 \mathrm{pp}$.

Anderson, T. 1910: The volcano of Matavanu in Savaii. Q. Jl geol. Soc. Lond. 66, 621-639.

Bartrum, J. A. 1930: Pillow-lavas and columnar fan-structures on Muriwai, Auckland, New Zealand. J. Geol. 38, 447-455.

Belliere, J. 1958: Contribution à l'étude pétrographique des schistes cristallins du Massif des Aiguilles Rouges (Hte Savoie). Annls Soc. géol. Belg. 81, 1-198.

Berg, R. R. 1952: Feldspathized sandstone. J. sedim. Petrol. 22, 221-223.

Berthelsen, A. 1958: On the chronology of the Ivigtut district, South Greenland. A compilation. GGU internal report, $10 \mathrm{pp}$.

- 1960: An example of a structural approach to the migmatite problem. Rep. 21 st int. geol. Congr., Norden, 14, 149-157.

- 1961: On the chronology of the Precambrian of western Greenland. In Rauscr, G. O. (edit.). Geology of the Arctic 1, 329-338, Toronto Press.

- 1962: On the geology of the country around Ivigtut, SW Greenland, Geol. Rdsch. 52, 269-280.

— , Bondesen, E. \& Jensen, S. B. 1962: On the so-called wildmigmatites. Krystalinikum 1, 31-50.

- \& Noe-Nygand, A. 1965: The Precambrian of Greenland. In Rankama, K. (edit.). The Precambrian 2, 113-262. Intersci. Publ., London \& New York.

BøGGILD, O. B. 1917: Handbuch der regionalen Geologie IV, 21, 2 a, Heidelberg, $37 \mathrm{pp}$.

BondeSEn, E. 1962: Grænseland kompleksets geologi. GGU internal report.

- 1970: The stratigraphy and deformation of the Precambrian rocks of the Grænseland area, South-West Greenland. Bull. Gronlands geol. Unders. 86 (also Meddr Gronland 185'), $210 \mathrm{pp}$.

- \& Henriksen, N. 1965: On some pre-Cambrian metadolerites from the central Ivigtut region, SW Greenland. Bull. Gronlands geol. Unders. 52 (also Meddr Gronland $\left.179^{2}\right), 42 \mathrm{pp}$.

- , Raunsgand Pedersen, K. \& Jørgensen, O. 1967: Precambrian organisms and the isotopic composition of organic remains in the Ketilidian of SouthWest Greenland. Bull. Gronlands geol. Unders. 67 (also Meddr Gronland 1644), $41 \mathrm{pp}$.

Breemen, O. van, Aftalion, M. \& Allaart, J. H. 1974: Isotopic and geochronologic studies on granites from the Ketilidian mobile belt of South Greenland. Bull. geol. Soc. Amer. 85, 403-412.

Bridgwater, D. 1965: Isotopic age determinations from South Greenland and their geological setting. Bull. Gronlands geol. Unders. 53 (also Meddr Grønland 1794), $56 \mathrm{pp}$. 
Bridgwater, D. \& HarRy, W. T. 1968: Anorthosite xenoliths and plagioclase megacrysts in Precambrian intrusions of South Greenland. Bull. Gronlands geol. Unders. 77 (also Meddr Gronland 185²), 67 pp.

Bridgwater, D., Escher, A. \& Watterson, J. 1973: Tectonic displacements and thermal activity in two contrasting Proterozoic mobile belts from Greenland. Phil. Trans. R. Soc. Lond. Ser. A, 273, 513-533.

Burrr, M. 1955: Notes de terrain, Eté 1955. GGU internal report.

Butterfield, J. A. 1936: Outgrowths on zircon. Geol. Mag. 73, 511-516.

Caldisen, K. 1943: Igneous rocks of the Ivigtut region, Greenland. Part I. The nepheline syenites of the Grønne Dal-Ika area. Meddr Gronland 131 ${ }^{8}, 74 \mathrm{pp}$.

Carlisle, D. 1963: Pillow breccias and their aquagene tuffs, Quadra Island, British Columbia. J. Geol. 71, 48-71.

Cuoos, E. 1946: Lineation. Mem. geol. Soc. Amer. 18.

- 1961: Bedding slips, wedges and folding in layered sequences. Bull. Comm. géol. Finl. 196, 105-122.

Collins, W. H. 1914: La géologie de la division minière de Gowgonda. Mem. geol. Surv. Can. 33, $141 \mathrm{pp}$.

- 1925: North shore of Lake Huron. Mem. geol. Sur,. Can. 143, 160 pp.

Dawes, P. R. 1970: The Plutonic history of the Tassiussaq area, South Greenland, with special reference to a high-grade gneiss complex. Bull. Gronlands geol. Unders. 88 (also Meddr Gronland 1893), $125 \mathrm{pp}$.

Dunbar, C. O. \& Rodgers, J. 1957: Principles of Stratigraphy. John Wiley and Sons, Inc., New York.

Emeleus, C. H. 1964: The Grønnedal-fka alkaline complex, South Greenland. The structure and geological history of the complex. Bull. Gronlands geol. Unders. 45 (also Meddr Gronland 172'), $75 \mathrm{pp}$.

Eskola, P. \& Nieminen, E. 1938: The quartzite area of Tiirismaa near Lahti. Bull. Comm. géol Finl. 123, 29-45.

Fisher, R. V. 1961: Proposed classification of volcaniclastic sediments and rocks. Bull. geol. Soc. Am. 72, 1409-1414.

Fourmarier, P. 1949: Principes de géologie. I \& II. Paris: Masson.

- 1951: L'influence des déplacements relatifs dans la production de la schistosité. Annls Soc. géol. Belg. 74, 255-260.

- 1953: L'origine du gaufrage des schistes. Annls Soc. géol. Belg. 77, B 23-26.

Froidevaux, C. \& Muller, J. 1958: Etude géologique et tectonique d'Arsuk Storø. GGU internal report.

Goldich, S. S. 1934: Authigenic feldspar in sandstones of south-eastern Minnesota. J. sedim. Petrol. 4, 89-95.

GRY, H. 1931: Notes de terrain citées par Callisen K. (1943).

HARкеR, A. 1960: Metamorphism. A study of the transformations of rock-masses. London: Methuen, $362 \mathrm{pp}$.

Henriksen, N. 1960: Structural analysis of a fault in South-West Greenland. Bull. Gronlands geol. Unders. 26 (also Meddr Gronland 1629), $40 \mathrm{pp}$.

- 1961: En geologisk beskrivelse af et område i S.W. Grønland. GGU internal report.

Henriksen, N. 1969: Boundary relations between Precambrian fold belts in the Ivigtut area, Southwest Greenland. Spec. Pap. geol. Ass. Can. 5, 143-154.

Hietanen, A. 1938: On the petrology of the Finnish quartzites. Bull. Comm. géol. Finl. 122, 1-118.

Higgins, A. K. \& BondeSEN, E. 1966: Supracrustals of pre-Ketilidian age (the Tartoq Group) and their relationships with Ketilidian supracrustals in the Ivigtut region, South-West Greenland. Rapp. Gronlands geol. Unders. 8, 21 pp. 
Kania, J. E. A. 1929: Precipitation of limestone by submarine vents, fumaroles, and lava flows. Am. J. Sci. 5 Ser. 18, 347-359.

Косн, L. 1929: The geology of East Greenland. Meddr Gronland 732 204 pp.

Köнler, A. 1950: Bemerkungen über Plagioklaszwillinge nach (15̄0) und (111) sowie über die Banater Verwachsung. Tschermaks mineralog. petrogr. Mitt. 3. Folge 1, $347-352$.

Kry nine, P. D. 1946: The tourmaline group in sediments. J. Geol. 54, 65-87.

Larsen, O. 1966: K/Ar age determinations from western Greenland. Rapp. Gronlands geol. Unders. 11, 57-67.

Lewis, V. J. 1914: Origin of pillow. Bull. geol. Soc. Am. 25, 591-654.

Millot, G. 1964: Géologie des argiles: altérations, sédimentologie, géochimie. $499 \mathrm{pp}$. Paris: Masson.

Misch, P. 1965: Radial epidote glomeroblast formed under conditions of synkinematic metamorphism. A new mechanism of collective crystalloblastesis. Geol. Rdsch. 54, 944-956.

Moorbath, S., Webster, R. K. \& Morgan, J. W. 1960: Absolute age determination in South-West Greenland. Meddr Grønland $162^{\circ}, 13 \mathrm{pp}$.

Moore, J. G. \& REed, R. K. 1963: Pillow structures of submarine basalts, East Hawaii. Prof. Pap. U.S. geol. Surv. 475-B, 153-157.

Muller, J. 1962: Prélèvement d'échantillons de 3 générations de dykes TD postGardar, présumés d'âge tertiaire, en vue d'une étude paléomagnétique. Côte $W$, Groënland méridional. GGU internal report.

Pedersen, K. Raunsgaard 1966: Precambrian fossils from the Ketilidian of SouthWest Greenland. Rapp. Gronlands geol. Unders. 11, 40-41.

- 1968: Fossils of Precambrian age from South-West Greenland. Rapp. Grønlands geol. Unders. 15, 51-53.

Pettisonn, F. J. 1957 : Sedimentary rocks. New York: Harper \& Bros., 718 pp.

Poulsen, V. 1964: The sandstones of the Precambrian Eriksfjord Formation in South Greenland. Rapp. Grønlands geol. Unders. 2, $16 \mathrm{pp}$.

Quirke, T. T. \& Collins, W. H. 1930: The disappearance of the Huronian. Mem. geol. Surv. Can. 160.

Reinhard, M. 1931: Universal Drehtischmethoden. Basel, 119 pp.

Rittmann, A. 1963: Les volcans et leur activité. Ed. franç. par Tazieff, H, Paris: Masson, $461 \mathrm{pp}$.

Salimi, F. 1965: Etude pétrographique des roches ophiolitiques des Préalpes romandes. Bull. Suisse Minér. Pétr. 45, 189-279.

Sampson, E. 1923: The ferruginous chert formation of Notre Dame Bay, Newfoundland. J. Geol. 31, 571-598.

SGHARBERT, H. G. 1963: A sandstone dyke in the Julianehåb granite of Qeqertarssuaq, Julianehåb district. Meddr dansk geol. Foren. 15, 183-188.

Sørensen, H. 1950: An examination of the plagioclases of some Hekla lavas. Meddr dansk geol. Foren. 11, 522-542.

- 1966: On the magma evolution of the alkaline igneous province of South Greenland. Rapp. Gronlands geol. Unders. 7, $19 \mathrm{pp}$.

Taliaferro, N. L. 1943 : Franciscan-Knoxville Problem. Bull. Am. Ass. Petrol. Geol. 27, 109-219.

Tester, A. C. \& Atwater, G. I. 1934: Occurrence of authigenic feldspar in sediments. J. sedim. Petrol. 4, 23-31.

Tröger, W. E. von 1959: Optische Bestimmung der gesteinsbildenden Minerale. Teil I. Bestimmungstabellen. Stuttgart: Schweizerbarth'Sache Verlag, $147 \mathrm{pp.}$

TURNER, F. J. \& VERHOOGEN, J. 1951: Igneous and metamorphic petrology. New York: McGraw-Hill, $602 \mathrm{pp}$. 
TURNer, F. J. \& Weiss, L. E. 1963: Structural analysis of metamorphic tectonites. New York: McGraw-Hill, 545 pp.

Ussing, N. V. 1912: Geology of the country around Julianehaab, Greenland. Meddr Gronland 38, $376 \mathrm{pp}$.

Vitanage, P. W. 1957: Studies of zircon types in Ceylon Precambrian complex. J. Geol. 65, 117-128.

Vuagnat, M. 1946: Sur quelques diabases suisses. Contribution à l'étude du problème des spilites et pillow-lavas. Bull. Suisse Minér. Pétr. 26, 116-228.

- 1948: Remarques sur 3 diabases suisses. Contribution à l'étude du problème des spilites et pillow-lavas. Bull. Suisse Minér. Pétr. 28, 263-273.

- 1949: Sur les pillow-lavas dalradiennes de la péninsule de Tayvallich (Argyllshire). Bull. Suisse Minér. Pétr. 29, 523-536.

- 1951: Le rôle des roches basiques dans les Alpes. Bull. Suisse Minér. Pétr. 31, 309-322.

- \& Pustaszeri, L. 1965: Réflexions sur la structure et le mode de formation des coulées en coussins du Montgenèvre, Htes Alpes. Archs Sci., Genève, 18, fasc. 3.

Walton, B. 1966 : Carbonatite-lamprophyre dykes of Mesozoic age. Rapp. Gronlands geol. Unders. 11, 37-38.

Wegmann, C. E. 1929: Beispiele tektonischer Analysen des Grundgebirges in Finland. Bull. Comm. géol. Finl. 87, 100-129.

- 1937: Le socle précambrien du Groenland méridional. C.r. hebd. Séanc. Acad. Sci., Paris, 204, 874-875.

- 1938: Geological investigations in southern Greenland. Part I. On the structural divisions of southern Greenland. Meddr Gronland 1132, $148 \mathrm{pp}$.

- 1939: Übersicht über die Geologie Südgrönlands. Mitt. naturf. Ges. Schaffhausen 16, 188-212.

- 1947: Notes sur quelques problèmes de la tectonique superposée. Bull. Comm. géol. Finl. 140, 223-238.

- 1948: Notes sur la chronologie des formations précambriennes du Groenland méridional. Eclog. geol. Helv. 40, 7-14.

- 1959: La flexure de la Driva et quelques problèmes structuraux des Calédonides Scandinaves. Norsk geol. Tidsskr. 39, 25-74.

- \& Schaer, J. P. 1962: Chronologie des déformations des filons basiques dans les formations précambriennes du Sud de la Norvège. Norsk geol. Tidsskr. 42, 371387.

Weidmann, M. 1964: Géologie de la région située entre Tigssaluk Fjord et Sermiligârssuk Fjord (partie médiane) SW-Groenland. Bull. Gronlands geol. Unders. 40 (also Meddr Gronland $169^{5}$ ), $146 \mathrm{pp}$.

Williams, M., Turner, F. J. \& Gilbert, C. M. 1955 : Petrography. An introduction to the study of rocks in thin sections. San Francisco: Freemann, $406 \mathrm{pp}$.

Wilson, G. 1961: The tectonic significance of small scale structures, and their importance to the geologist in the field. Annls. Soc. geol. Belg. 84, 423-548.

Wilson, M. E. 1942: Structural features of the Keewatin volcanic rocks of western Quebec. Bull. geol. Soc. Am. 53, 53-70.

- 1960: Origin of pillow structure in early Precambrian lavas of western Quebec. J. Geol. 68, 97-102.

- 1962: Rouyn-Beauchastel map areas Quebec. Mem. geol. Surv. Can. 315, 118 pp.

WinCHeLl, A. N. \& H. 1959: Elements of optical mineralogy. An introduction to microscope petrography. Part II. Descriptions of the minerals. (4 édit.). New York: Wiley, $581 \mathrm{pp}$.

WindeEy, B. F. 1967: On the classification of the West Greenland anorthosites. Geol. Rdsch. 56, 1020-1026. 
Windey, B. F., Hentiksen, N., Higgins, A. K., Bondesen, E. \& Jensen, S. B. 1966: Some border relations between supracrustal and infracrustal rocks in South-West Greenland. Rapp. Gronlands geol. Unders. 9, 43 pp.

WIN KLER, H. G. F. 1967: Petrogenesis of metamorphir rocks. (2nd edit.). New York: Springer-Verlag, $237 \mathrm{pp}$.

WooD, D.S. 1964: Some structures in the Dalradian pillow-lavas of the Tayvallich Peninsula, Argyll. Geol. Mag. 101, 481-487.

ZWART, H. J. 1960: Relations between folding and metamorphism in the central Pyrénées and their chronological succession. Geol. Mijnboux, 39, 163-180. 


\section{PLANCHES}




\section{Planche 1}

\section{Planche 1 a}

Microphotographie d'un orthoquartzite de la Formation de Taylers Havn; partie supérieure de l'horizon des quartzites à Taylers Fjeld. Le quartz est recristallisé en plages équidimensionnelles suturées. L.P. (× 15), GGU 38874.

\section{Planche $1 \mathrm{~b}$}

Microphotographie d'un quartzite de la Formation de Taylers Havn sur l'îlot Evqitsut. Les grains de quartz de 0,2 à $0,7 \mathrm{~mm}$ de diamètre sont noyés dans une matrice constituée de quartz, séricite, carbonate et un peu de feldspath. L.P. $(\times 20)$, GGU 54859 .

\section{Planche 1c}

Microphotographie d'un quartzite feldspathique de la Formation de Taylers Havn; partie inférieure de l'horizon des quartzites à Taylers Fjeld. Les feldspaths potassiques sont allongés parallèlement au clivage de la roche. Ces feldspaths, colorés au cobaltonitrite, ont une teinte grise sur la microphotographie. L.N. $(\times 20)$, GGU 38877. 

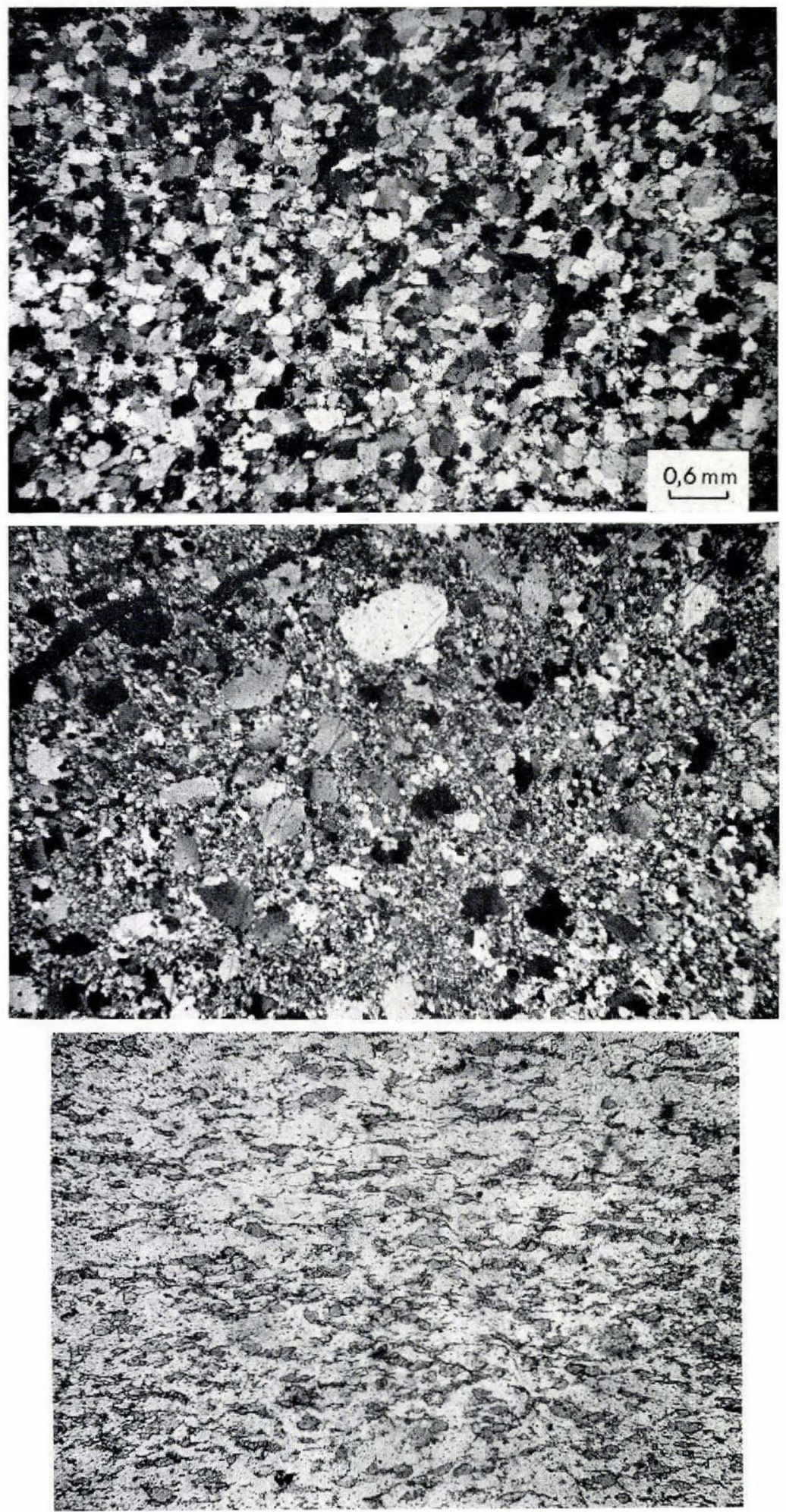


\section{Planche 2}

Planche 2a

Microphotographie d'un quartzite feldspathique de la base de l'horizon des quartzites à Taylers Fjeld. Phénocristal de feldspath potassique isolé dans une matrice constituée principalement de quartz. Le feldspath est entouré d'une "auréole mylonitique " de quartz, feldspath et séricite. Fissures tardives dans la partie droite du feldspath recoupant également la matrice. L.P. $(\times 20)$, GGU 38878.

\section{Planche $2 \mathrm{~b}$}

Microphotographie d'un phyllite quartzofeldspathique de l'horizon des quartzites dolomitiques à Taylers Havn. Recristallisation de quartz "discordant et lamellaire " en lits lenticulaires disposés parallèlement à la schistosité de la roche. L.P. $(\times 40)$, GGU 54874 .

\section{Planche 2c}

Microphotographie d'un schiste granuleux feldspathique de l'horizon des schistes sombres de la Formation de Taylers Havn, à Taylers Havn. Les plagioclases, de diamètre voisin de $1 \mathrm{~mm}$, sont cataclasés, fragmentés et noyés dans une matrice constituée de séricite, chlorite, feldspath granulé et saussuritisé et quartz. L.N. (× 35), GGU 38714. 

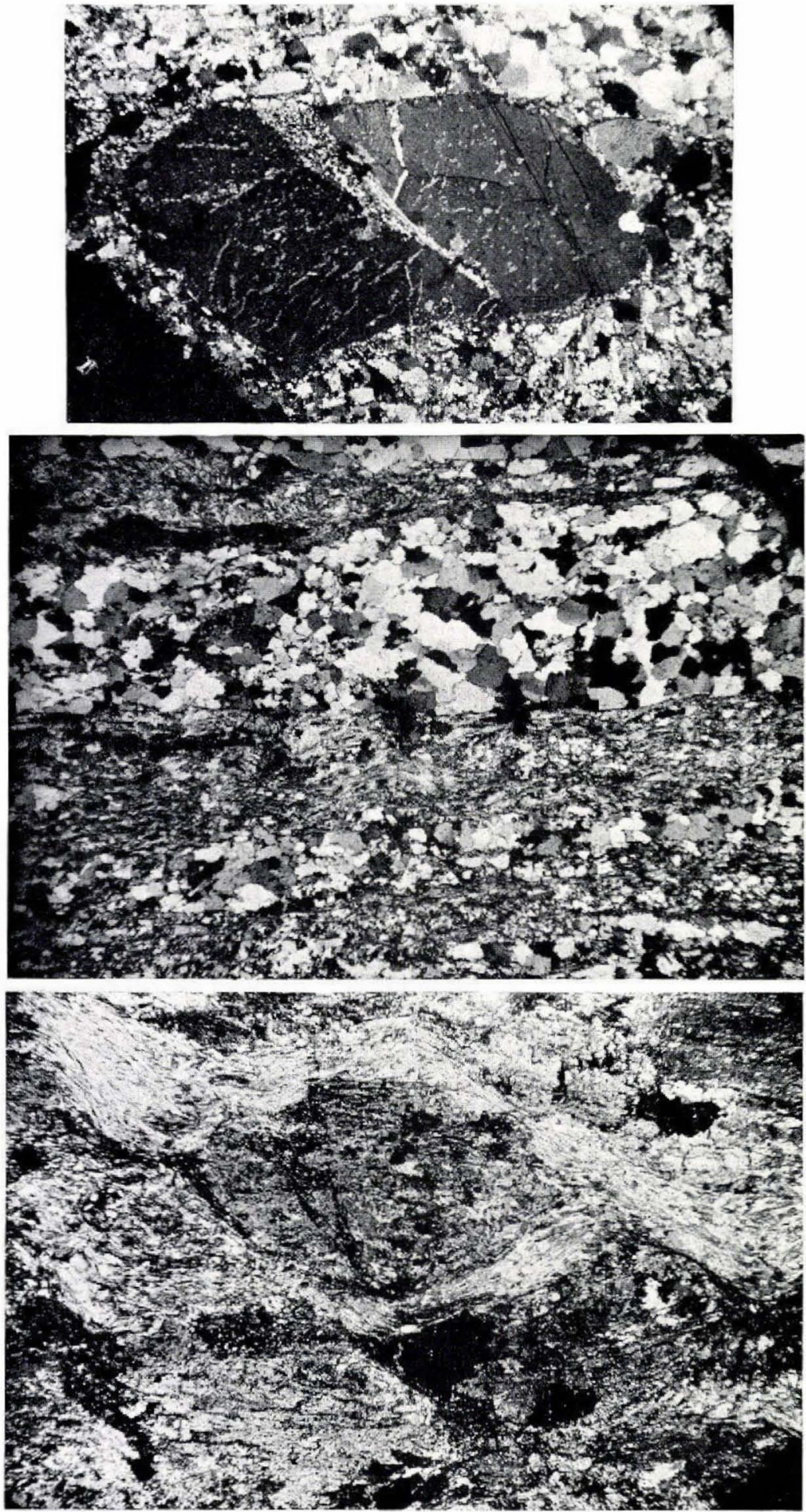


\section{Planche 3}

Planche 3a

"Intersections " entre la schistosité et le clivage dans les schistes sombres rubanés de la côte Ouest de Taylers Havn, au Nord d'Itivsâq. Ces «intersections " sont déformées par des petits plis appartenant à la structure synclinale N-S de Taylers Havn. L'échelle est donnée par des allumettes.

Planche $3 b$

Microphotographie d'un schiste sombre à biotite et grenat de l'horizon des schistes sombres de la Formation de Taylers Havn à Inugsuk. Développement de grenat postérieur à celui de la biotite brune. Cette biotite est elle-même postérieure à la formation de la schistosité $S_{1}$ et du clivage $S_{2}$ dont elle mime les structures. L.N. (× 25), GGU 56759 .

\section{Planche $3 \mathrm{c}$}

Quartzites phylliteux rubanés de la Formation d'Inugsugtût, sur la côte Ouest de Storø. La stratification, mise en évidence par le rubanement (lits sombres quartzophylliteux et lits clairs quartzitiques), est recoupée par une schistosité oblique. Cette schistosité est repliée par des plis E-W, plongeant de $10^{\circ}$ à $20^{\circ}$ vers $l^{\prime} \mathrm{W}$ ( $2^{\mathrm{e}}$ phase). 


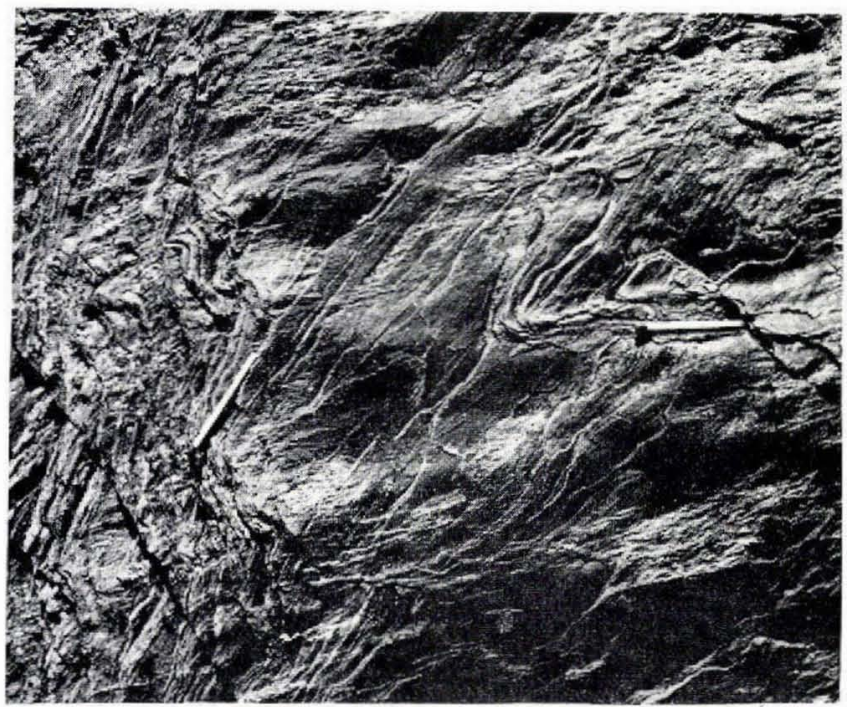

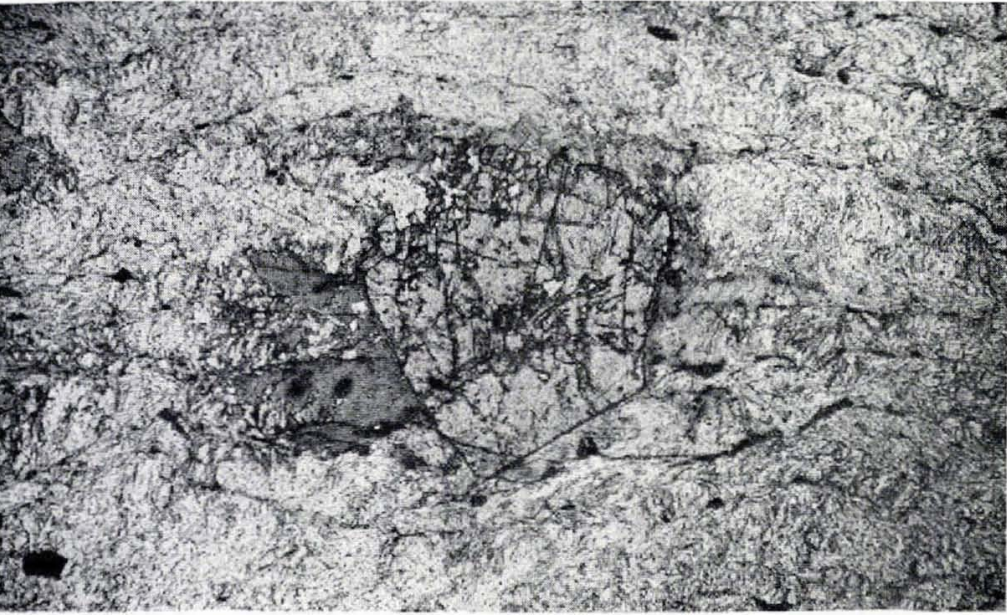

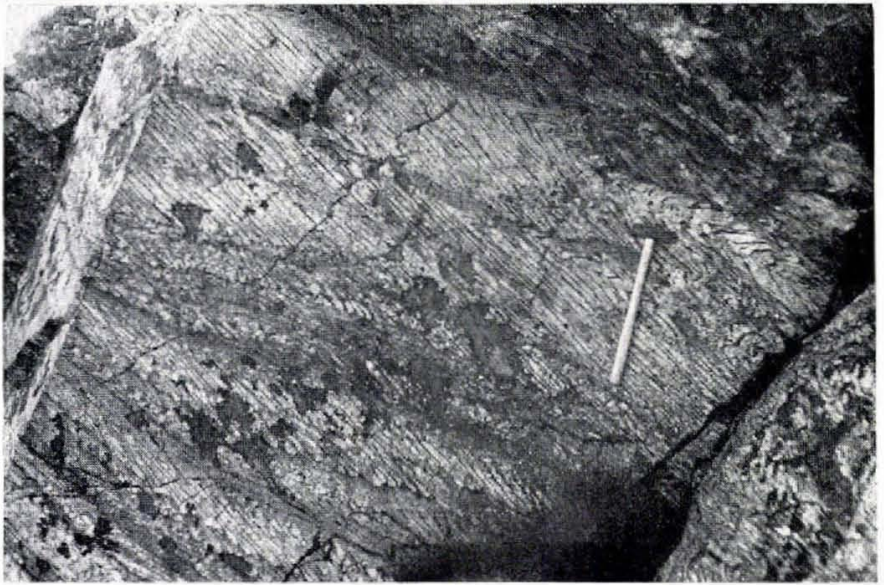




\section{Planche 4}

\section{Planche $4 \mathrm{a}$}

Microphotographie d'un phyllite rubané de la Formation d'Isua, sur la côte Est d'Arsuk $\varnothing$, au Nord d'Isua. Lits sériciteux et chloriteux alternant avec des lits quartzochloriteux plus clairs. Le rubanement correspond à la schistosité. Cette schistosité est plissotée avec formation, localement, d'un clivage. L.N. $(\times 10)$, GGU 32644.

\section{Planche $4 \mathrm{~b}$}

Superposition de structures métachrones dans les phyllites de la Formation d'Isua, à Isua, côte Est d'Arsuk $\varnothing$. Plis contemporains de la schistosité (1 ${ }^{\text {re }}$ phase) repliés par des plis de la $2^{\mathrm{e}}$ phase. Voir également la figure 40 dans le texte.

\section{Planche $4 \mathrm{c}$}

Microphotographie d'un gabbro-diorite intercalé dans les coulées de pillow-lavas de l'unité inférieure du Groupe d'Arsuk, à Krabbenæs, côte Ouest d'Arsuk $\varnothing$. Actinote poecilitique englobant des lattes de plagioclase saussuritisé. L.N. $(\times 25)$, GGU 32753. 

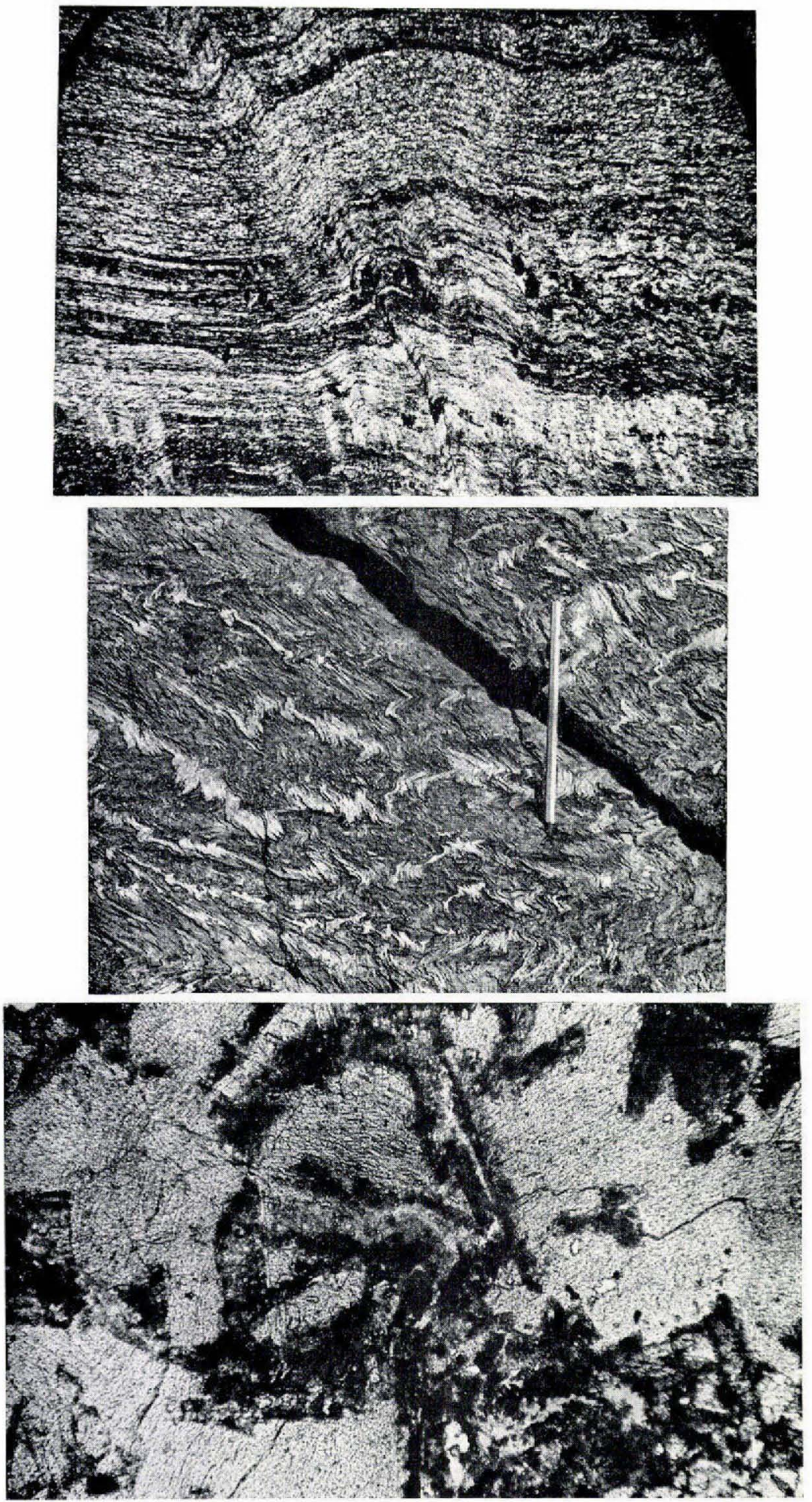


\section{Planche 5}

Section polie et laquée de pillow-lavas entassés les uns sur les autres et reliés entre eux. Groupe volcanique d'Arsuk, sur la côte Nord-Est de Storø. La liaison entre le gros pillow supérieur et le pillow sous-jacent de droite est visible dans la partie inférieure droite de la figure. La croûte (gris clair) entourant les deux pillows se termine à cet endroit. Le pillow supérieur correspond donc à un gros bourrelet du pillow sousjacent. Lorsque ce bourrelet s'est mis en place, le matériel quartzeux et tufacé (chert et boue siliceuse?), remplissant la petite dépression entre les deux pillows inférieurs, a été déformé. 


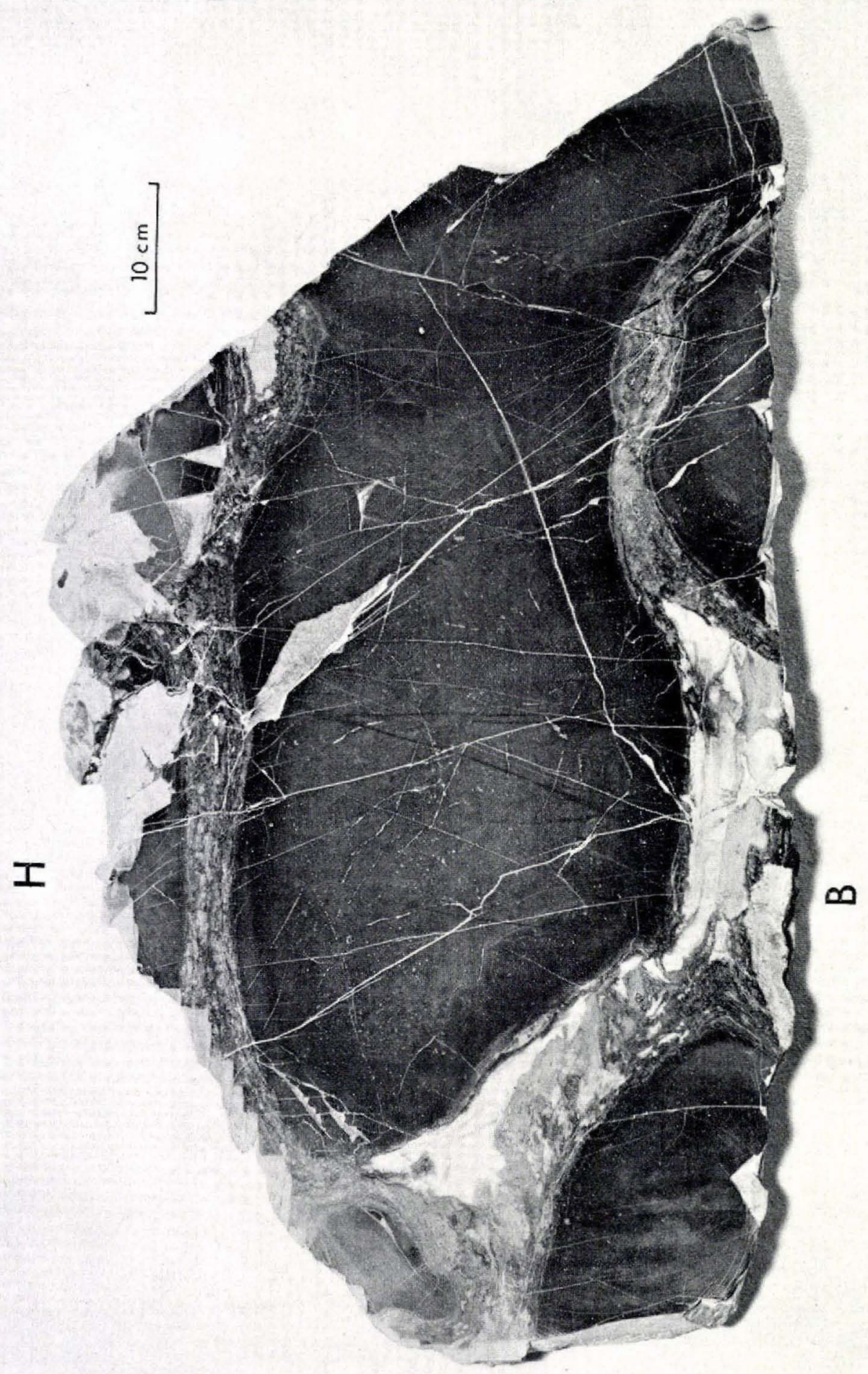




\section{Planche 6}

Section polie et laquée d'un pillow-lava de type ellipsoïdal à pédoncule basal. Unité inférieure du Groupe volcanique d'Arsuk à Pinguligssuánguaq, pointe Nord-Ouest d'Arsuk $\varnothing$. Les fentes de retrait convergent vers le pédoncule. Variation de texture et de teinte de la roche depuis le pédoncule jusqu'à la croûte supérieure du pillow. Voir également la planche 11. 


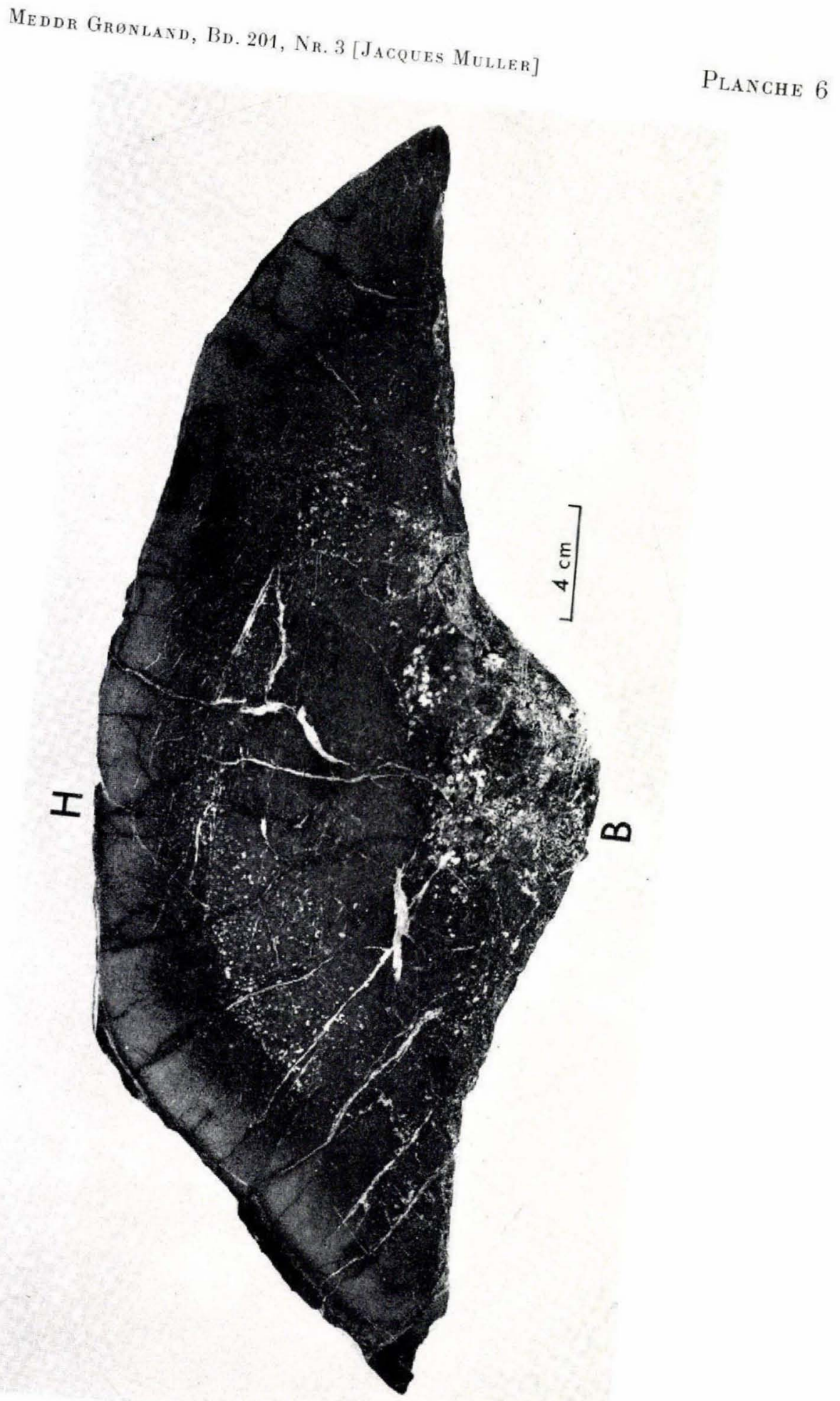




\section{Planche 7}

\section{Planche $7 \mathrm{a}$}

Section polie et laquée d'un pillow-lava coupé perpendiculairement à son grand axe. Unité inférieure du Groupe volcanique d'Arsuk à Manîtsoq. Fentes de retrait convergeant vers la cavité qui se trouve dans la moitié supérieure du pillow. Fracturation tardive avec remplissage de calcite. Dans la partie inférieure de la figure on observe de petites fractures parallèles qui sont liées à la déformation du pillow. Voir également les planches 8 a et 10 .

\section{Planche $7 \mathrm{~b}$}

Pillow-lava très déformé. Unité moyenne du Groupe d'Arsuk sur la côte Est de Manîtsoq. La croùte du pillow, marquée par un liseré sombre à peine visible, a été soulignée par de petits traits blancs. Les bordures du pillow sont affectées de schistosité tandis que la partie centrale est recoupée par des fractures de tension. Formation de fentes pennées à la limite de ces deux zones. Dans la moitié supérieure du pillow se trouve une cavité remplie secondairement par du quartz et de l'épidote. La base du pillow est indiquée par la flèche. 


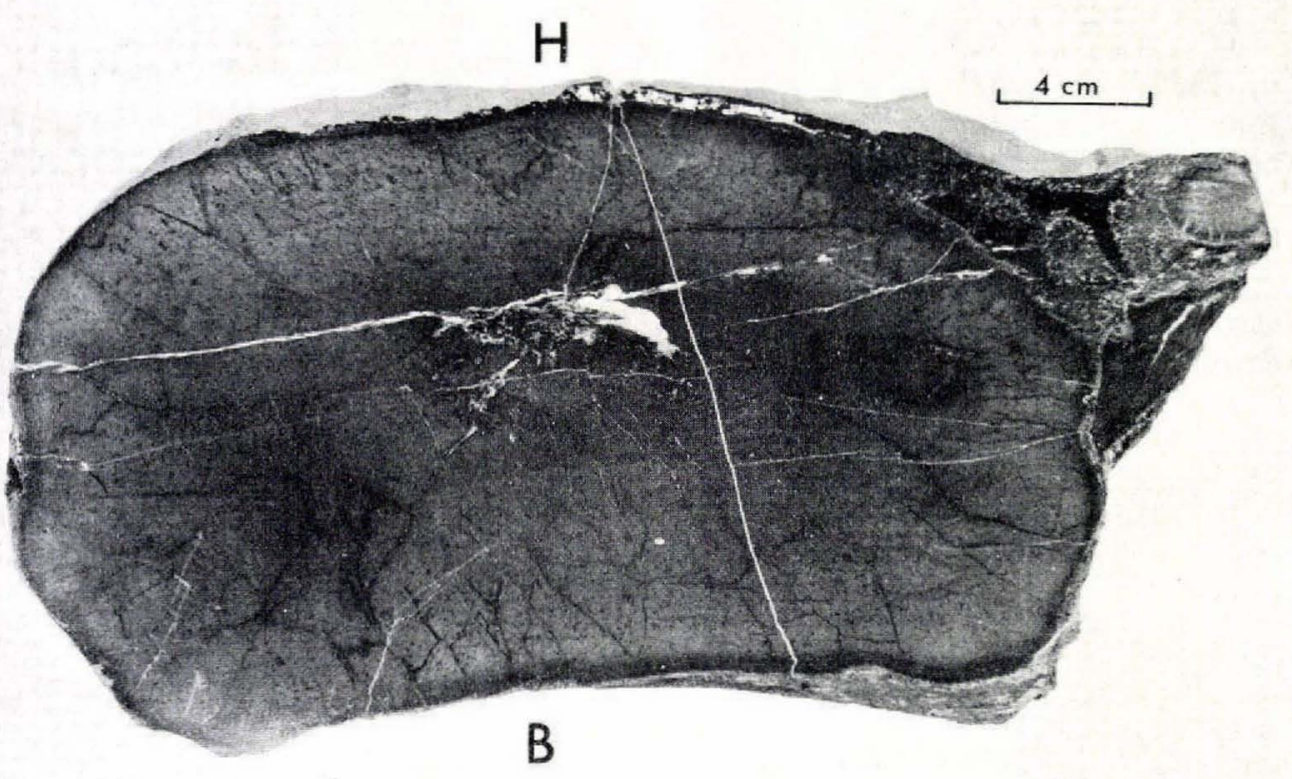

$a$

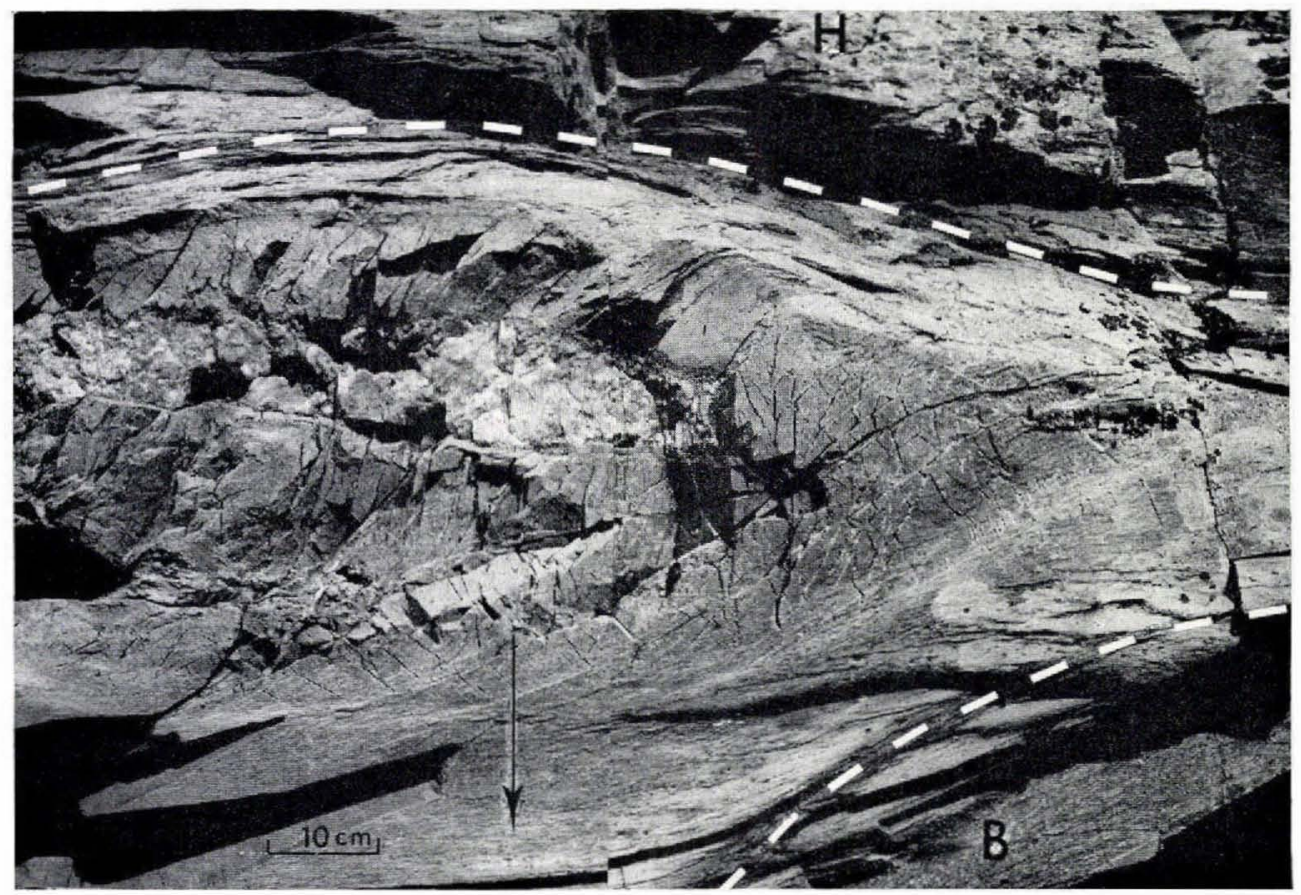




\section{Planche 8}

Planche 8 a

Figure de déformation du pillow-lava précédent. Cette section est perpendiculaire à celle de la planche $7 \mathrm{a}$. Fractures plus ou moins parallèles découpant le pillow en tranches. Le glissement des tranches les unes sur les autres permet l'allongement et l'aplatissement du pillow. La courbure des tranches est due à un aplatissement plus important du pillow dans sa partie la moins épaisse.

\section{Planche $8 \mathrm{~b}$}

Section polie et laquée d'un morceau de pillow de type ellipsoïdal. Unité inférieure du Groupe d'Arsuk à Manîtsoq (Itive kangigdleq). Vésicules dans la partie centrale du pillow (à droite sur la figure). Variation concentrique de texture et de teinte de la roche. La bordure sombre correspond à la zone sphérolitique; la bordure claire (à gauche sur la figure) correspond à la croûte. 

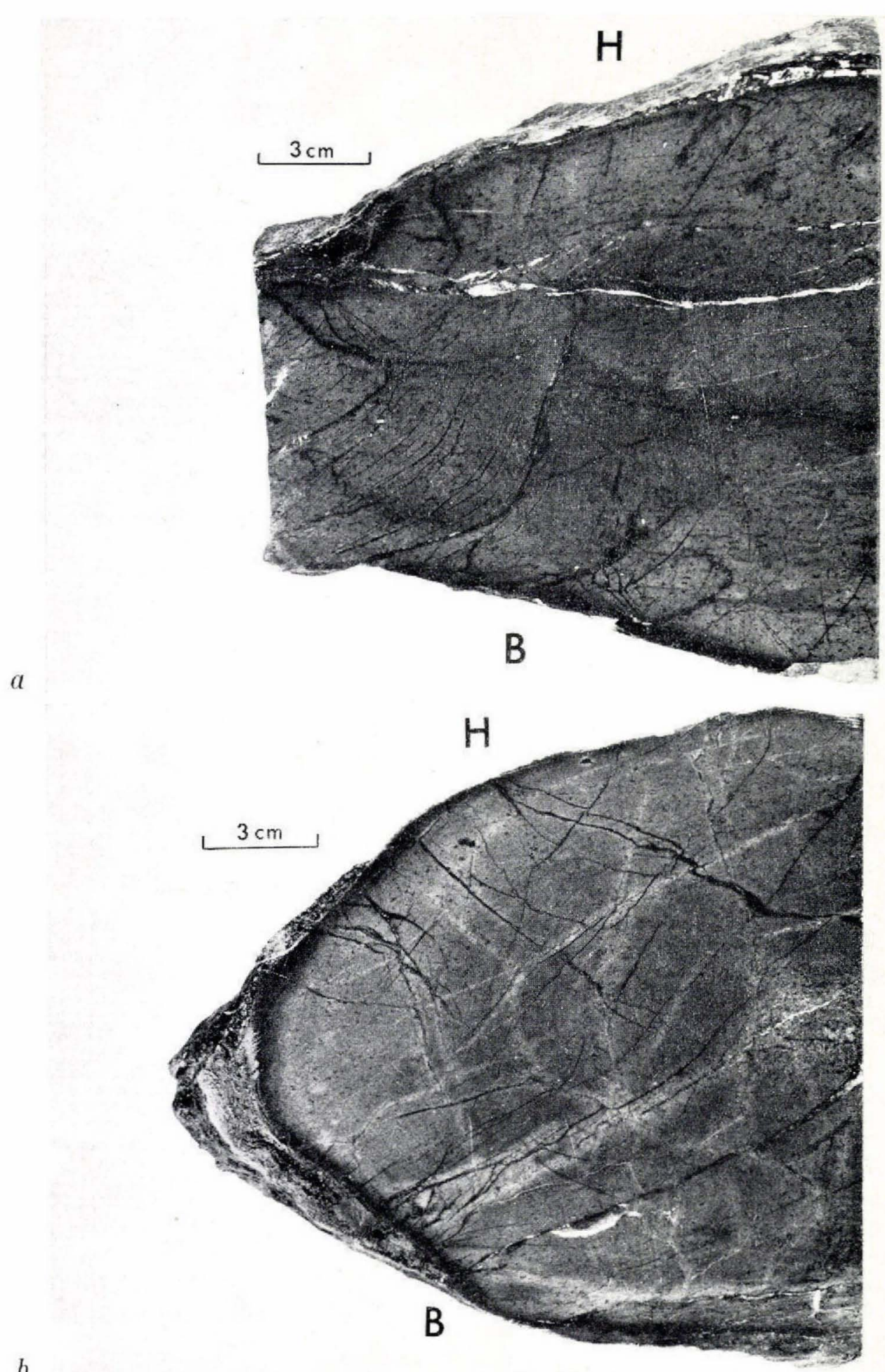


\section{Planche 9}

Coulée de pillows bréchiques. Unité inférieure du Groupe d'Arsuk à Blåbærelv, côte Nord d'Arsuk $\varnothing$. Les petits fragments partiellement bordés de croûte proviennent de l'éclatement de pillows. Le matériel interstitiel est constitué de tuf. 


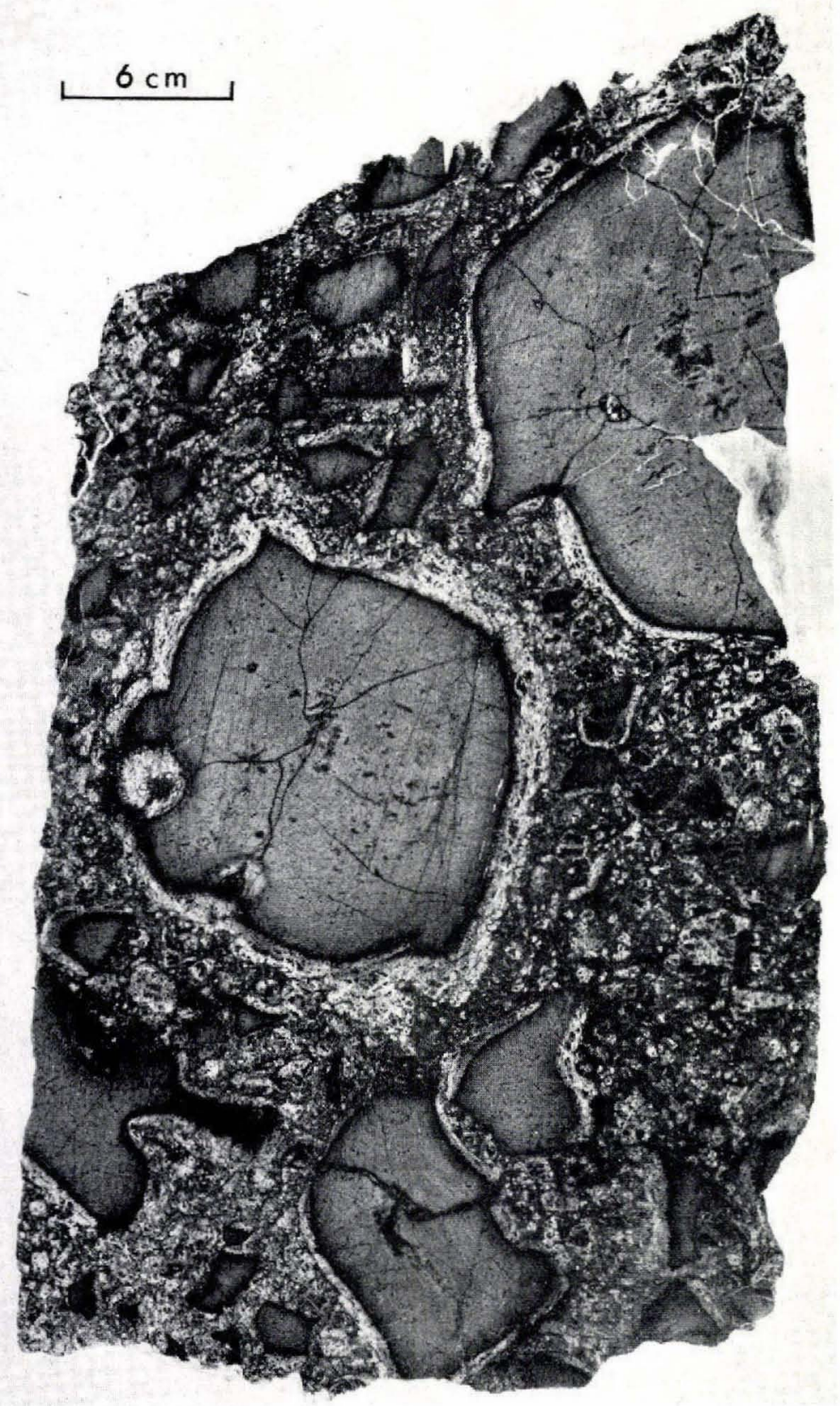




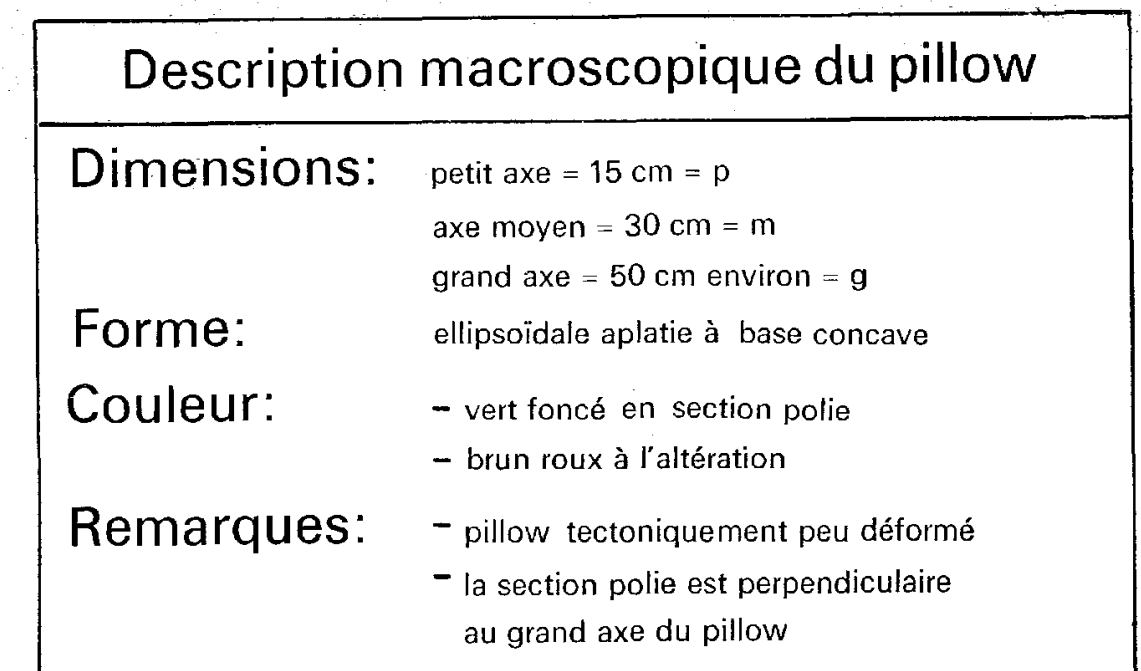

Liaison entre deux pillow-lavas par pédoncule latéral

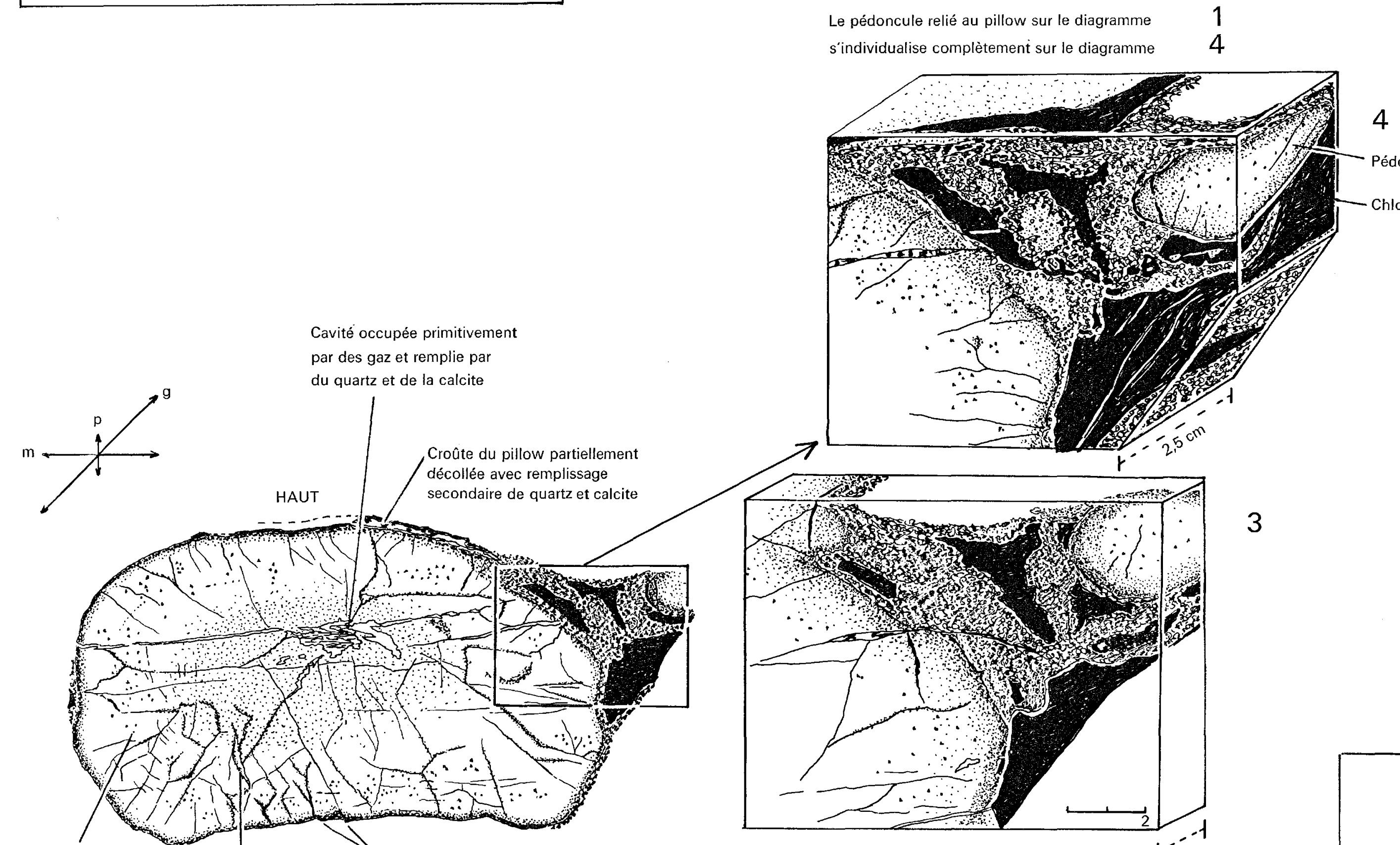

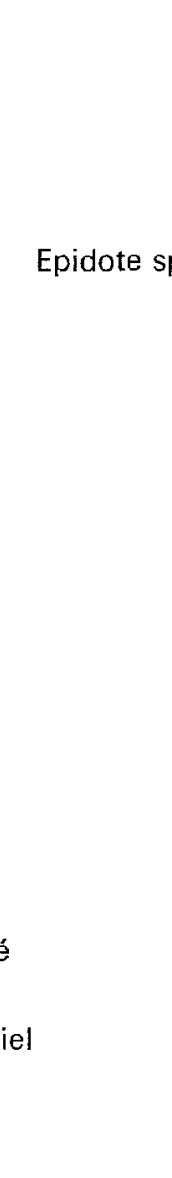

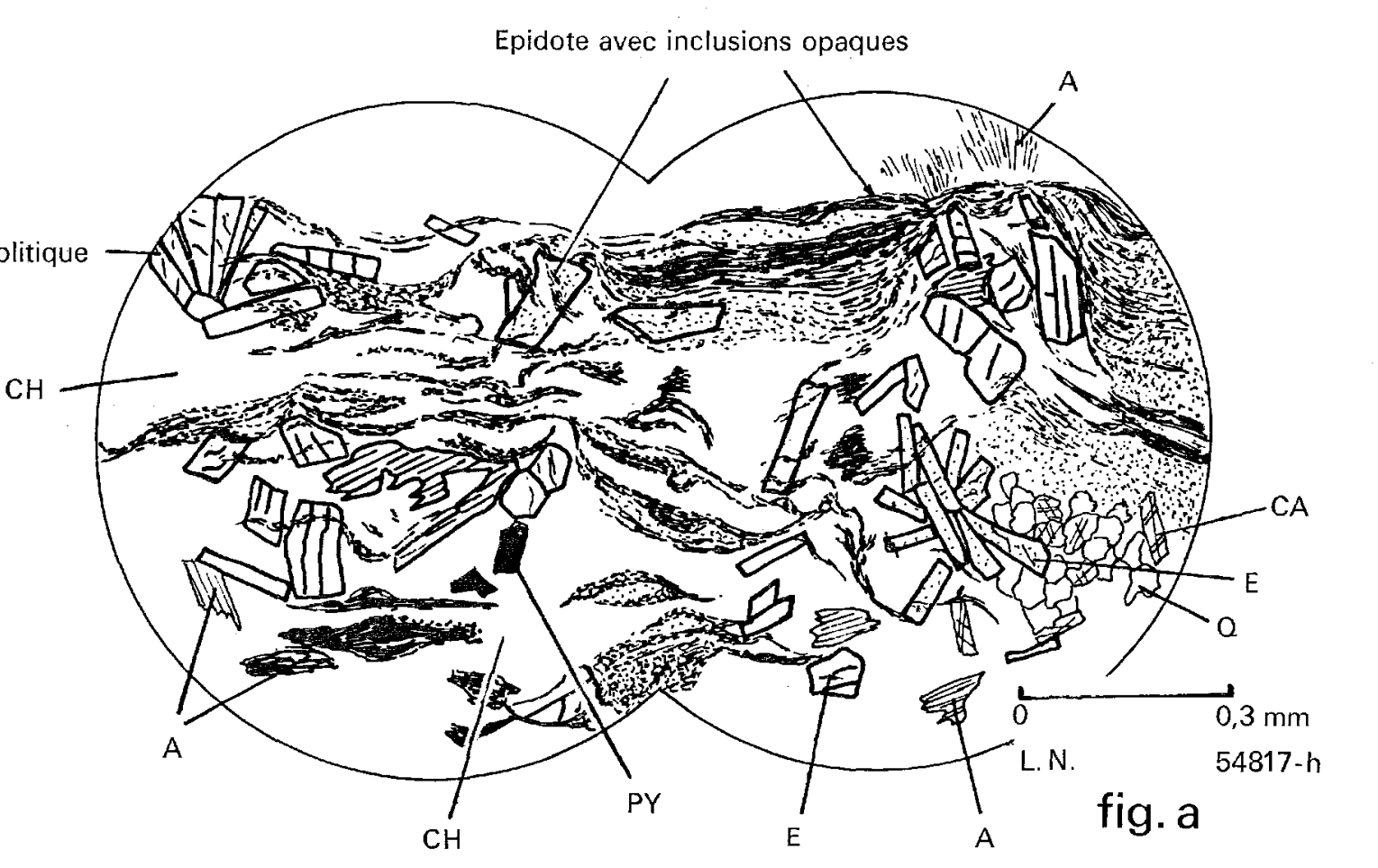

Croûte du pillow TEXTURE PSEUDO-FLUIDALE due à la transformation de la
matière cryptocristalline formant la croute (chlorite) Déveleppement postérieu
spherolites d'épidote
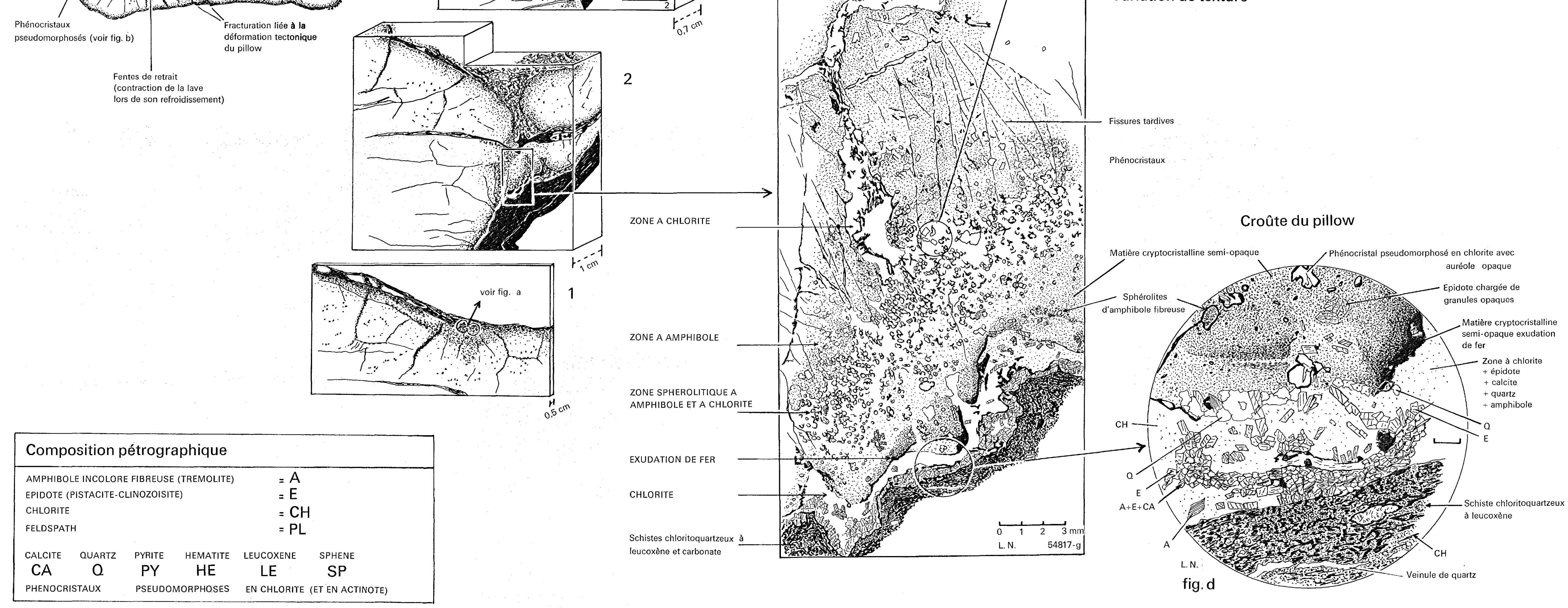


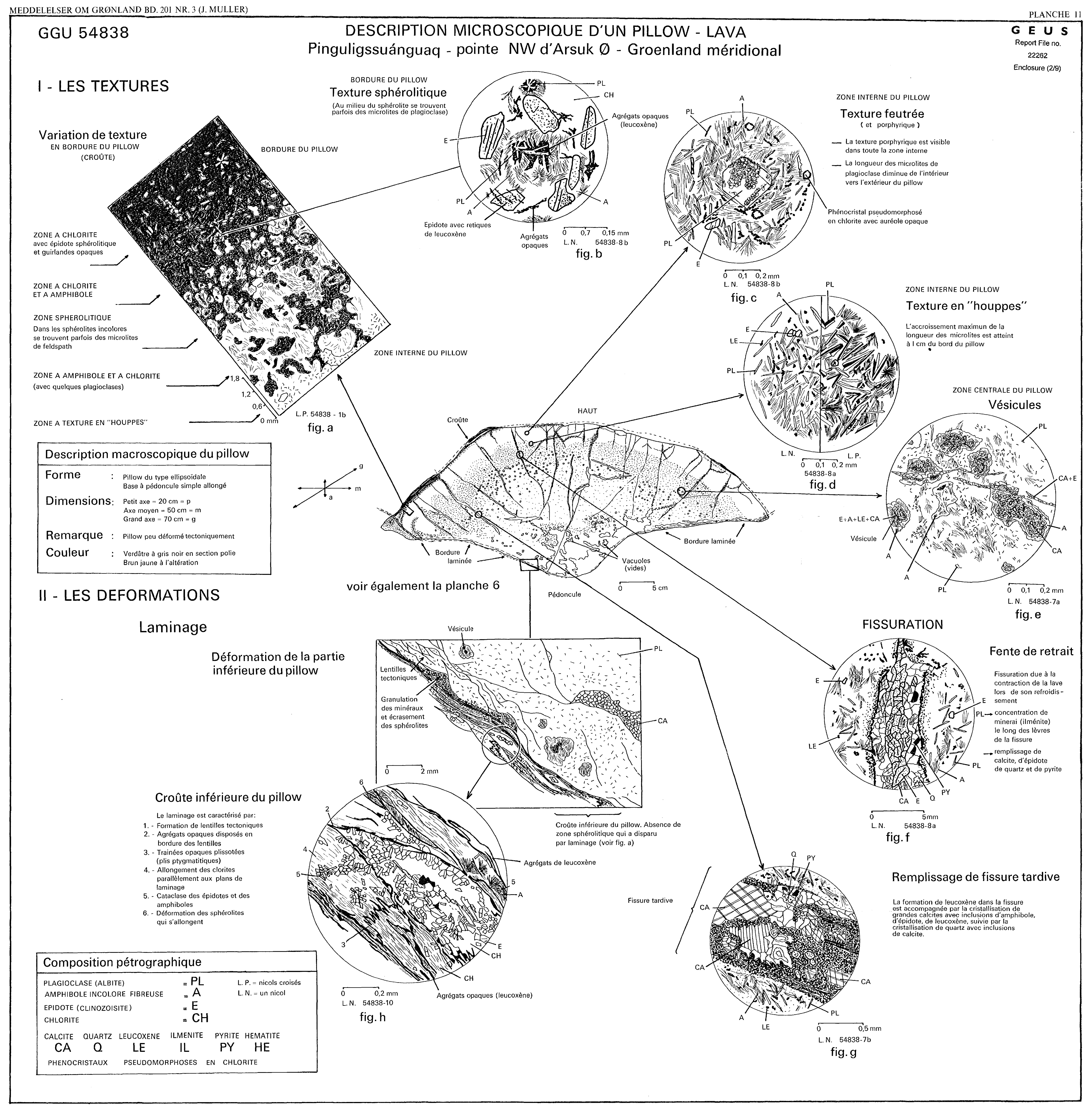




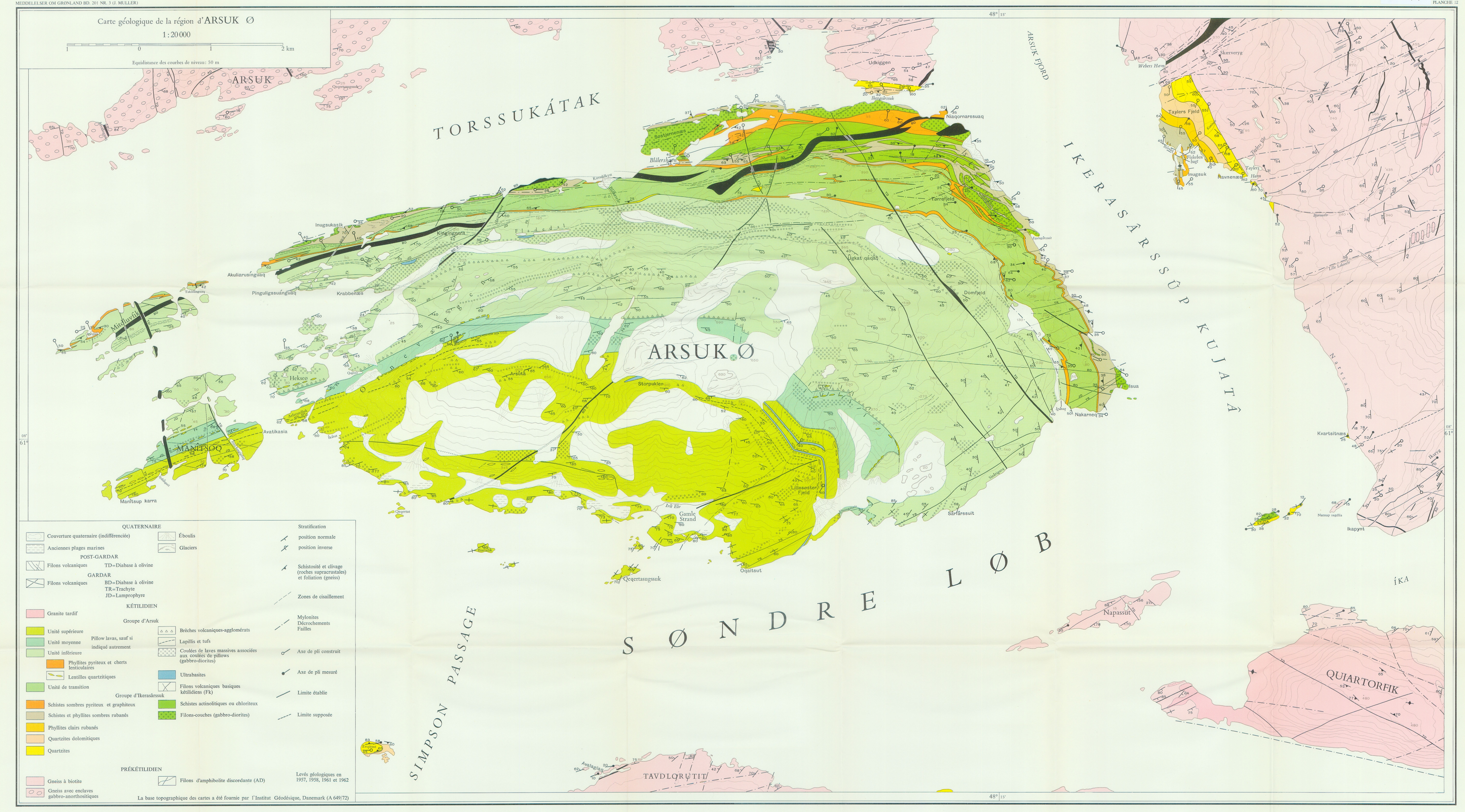


GRøNLANDS GEOLOGISKE UNDERSøGELSE




GRONLANDS GEOLOGISKE UNDERSØGELSE
SERVICE GEOOOGGOUE DU GROENIAND

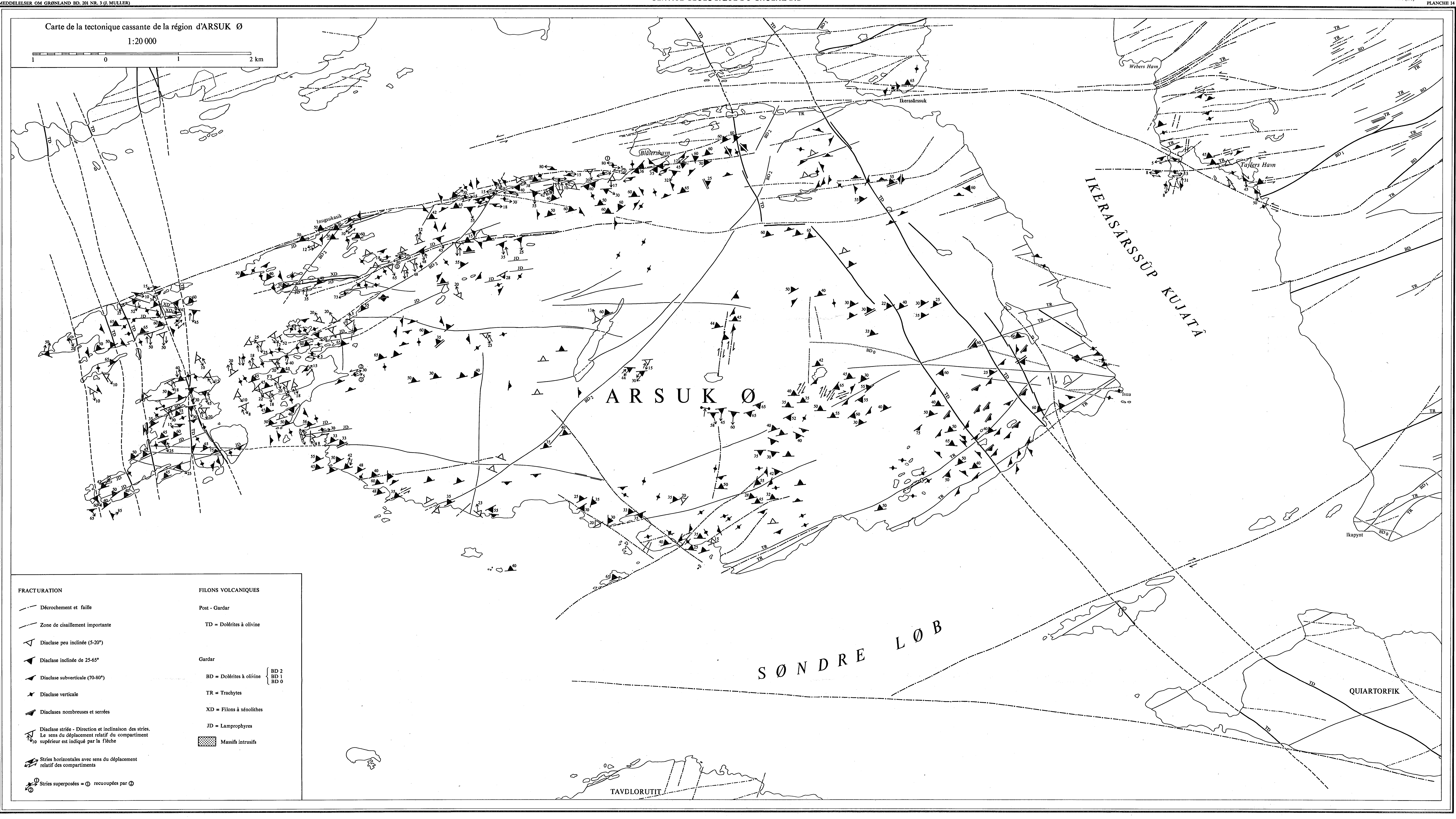




\section{ANALYSE STRUCTURALE DU SECTEUR D'ISUA}

COTE EST D'ARSUK $\emptyset$

Plis contemporains de la schistosité $\left(F_{1}\right)$

- Axe de pli mesuré (de 1 à $10 \mathrm{~cm}$ d'amplitude)

- - Axe de pli incliné, vertical, horizontal

Plis déformant la schistosité $\left(F_{2}\right)$

o Axe de pli mesuré (de 5 à $30 \mathrm{~cm}$ d'amplitude)
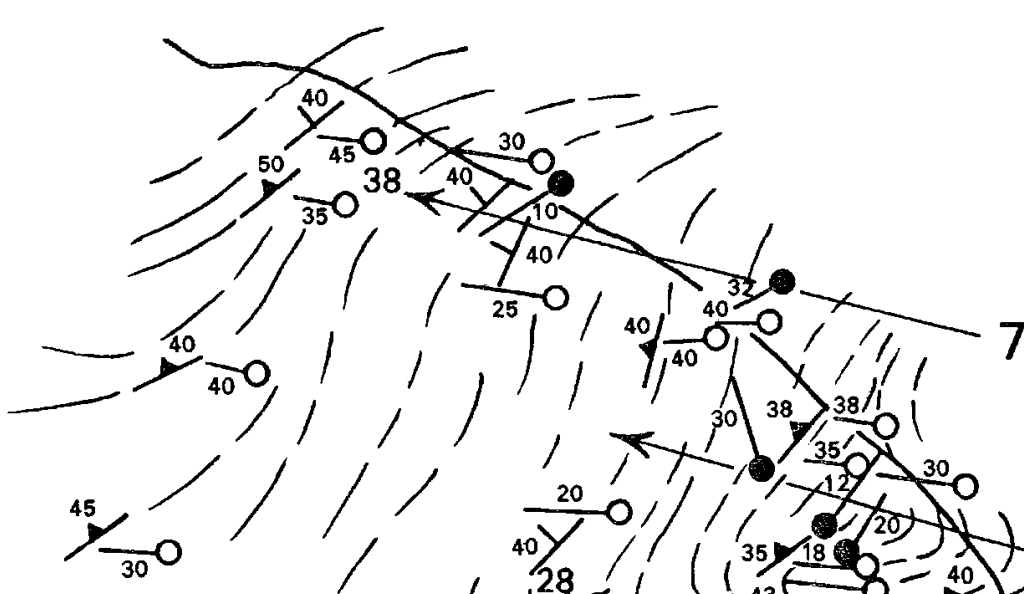

\section{LEGENDE DES STEREOGRAMMES}

Pôle des plans de stratification Intersection entre stratification
et schistosité

- Axe de pli $F_{1}$, mesuré

- Axe de pli $F_{1}$, construit

- Axe de pli $F_{2}$, mesuré

O- Axe de pli $F_{2}$, construit

$>$ Axe de boudinage
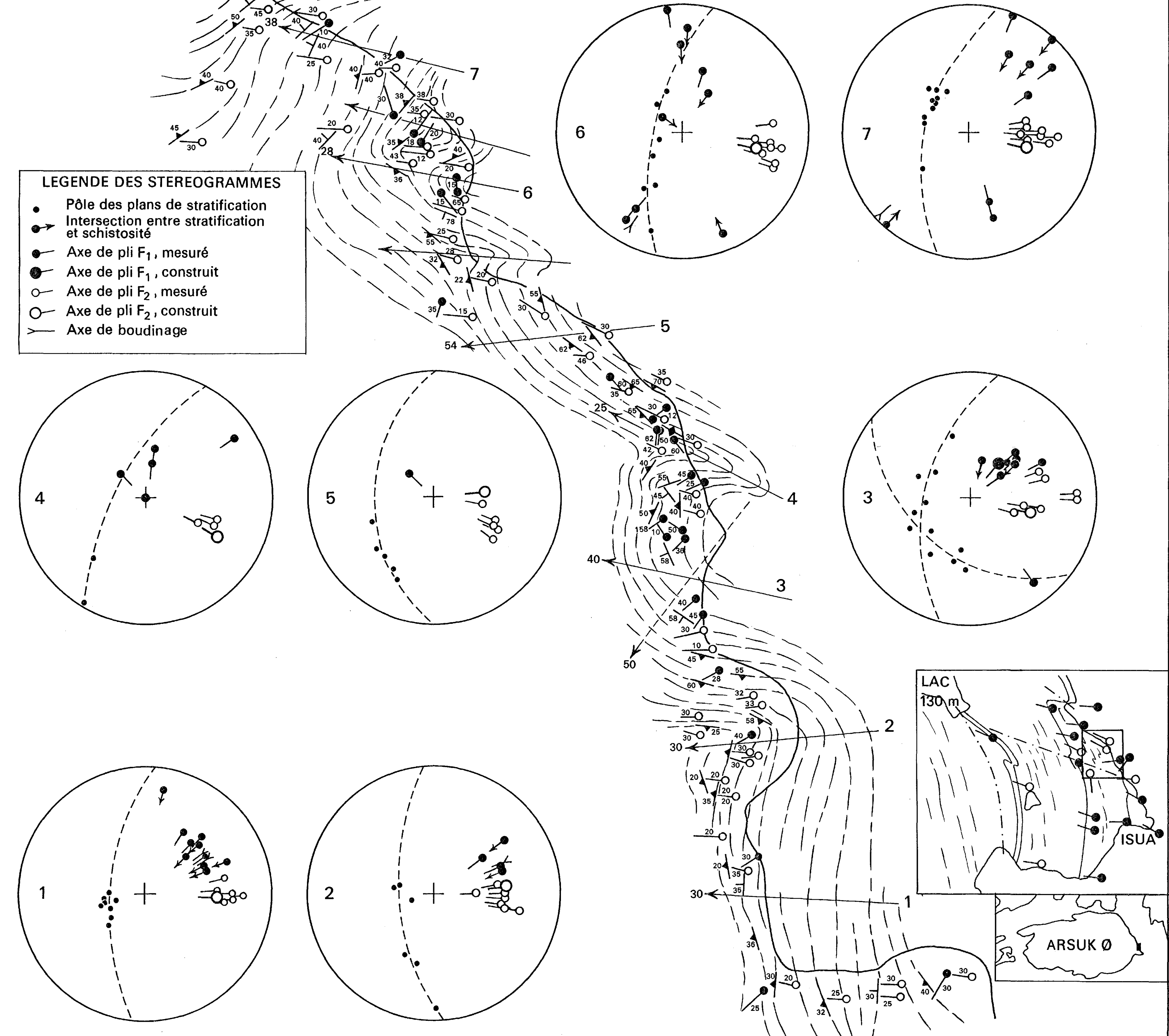


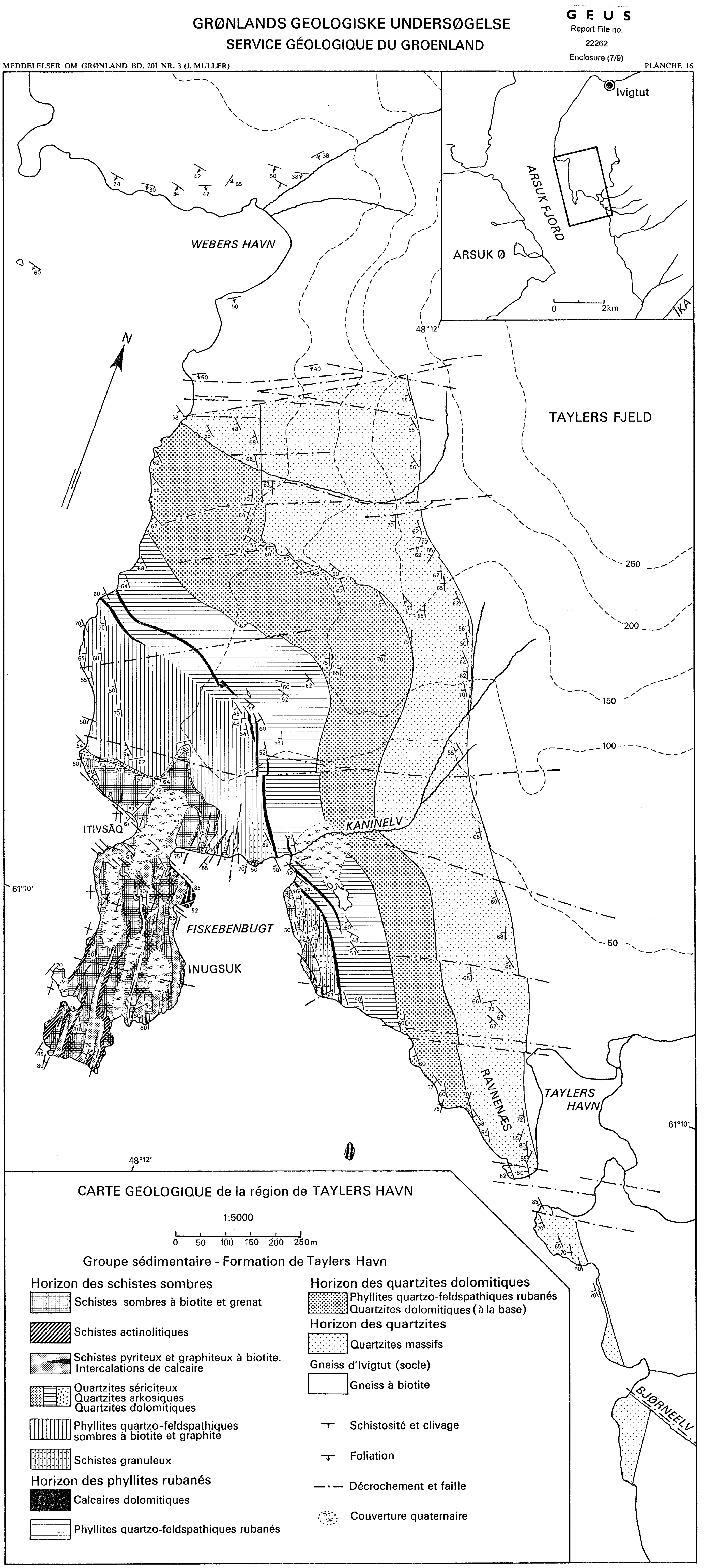




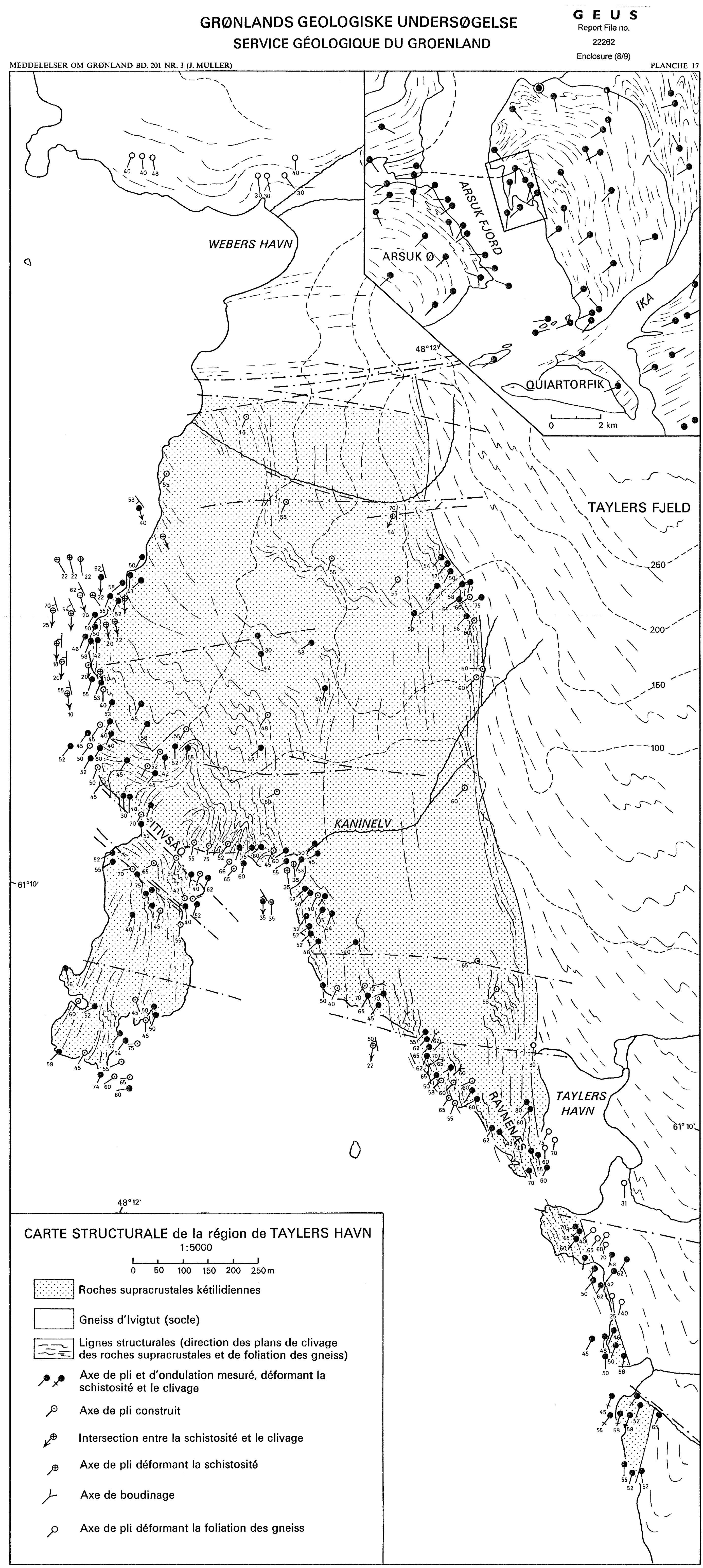




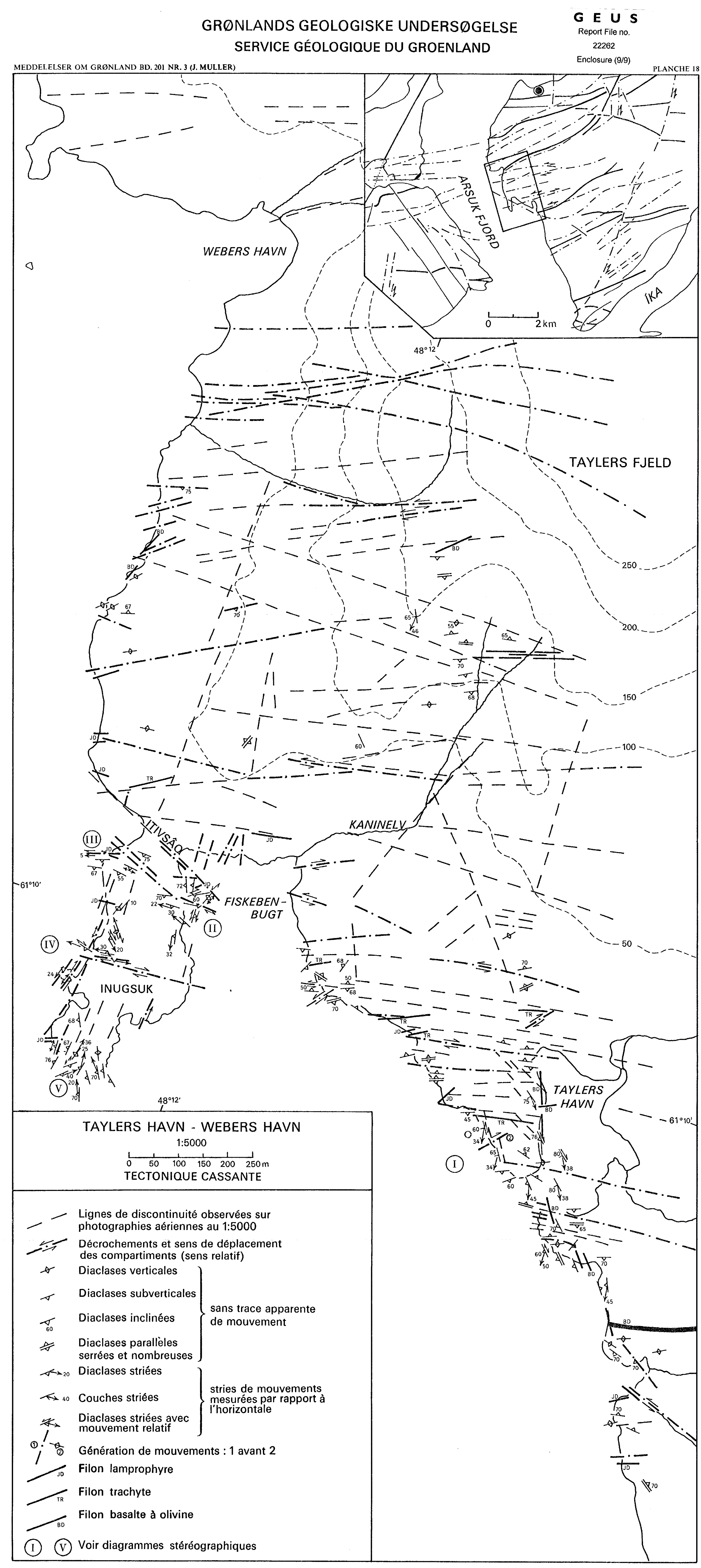

